\title{
PHYSICAL AND COMPUTATIONAL MODELS OF FREE SURFACE RELATED DEFECTS IN LOW-PRESSURE DIE-CAST ALUMINUM ALLOY WHEELS
}

$$
\text { by }
$$

\author{
Jianglan Duan
}

B.A., Huazhong University of Science and Technology, 2008

\section{A THESIS SUBMITTED IN PARTIAL FULFILLMENT OF THE REQUIREMENTS FOR THE DEGREE OF}

\section{MASTER OF APPLIED SCIENCE}

in

The Faculty of Graduate Studies

(Materials Engineering)

THE UNIVERSITY OF BRITISH COLUMBIA

(Vancouver)

November 2011

(C) Jianglan Duan, 2011 


\begin{abstract}
The difference of die filling, which can be characterized by the free surface flow behavior, has a strong influence on the quality of casting components. In the case of cast aluminum alloy wheels, an undesired filling pattern with excessive turbulence can cause portions of the surface oxide film to be entrained within the bulk liquid resulting in defects such as cosmetic paint-pops, hot tears, porosity and rim-leaks.
\end{abstract}

To investigate the influence of die filling on defect formation in low-pressure die-cast aluminum wheels, a water analogue physical model was built, instrumented and tested to simulate the free surface behavior during die filling of a low-pressure die-cast (LPDC) wheel. The physical model contains a transparent planar die section which was manufactured out of the geometry of a production die, and an automatic pressure control system that achieves liquid feeding conditions similar to the industrial process. A set of die filling tests with different venting conditions was carried out to explore the role of venting on the free surface behavior of water and to produce data for validation of a computational model. The computational model was developed, based on the commercial computational fluid dynamics code ANSYS CFX, for the purposes of predicting the flow conditions during die filling, providing qualitative and quantitative flow information that are otherwise not possible to obtain through experimental measurement, and identifying key features that influences the flow during die filling at a lower cost of time and labor.

Comparison between the experimental and numerical data has shown that the computational model was able to qualitatively reproduce the flow behavior observed in the water model in the conditions tested. Both the experimental and the model results indicate that the entrainment of surface oxide films and air bubbles could occur at the outboard rim flange during the filling of the flange, below the free surface of the returning waves in the spoke and at the junction of the 
hub and the spoke during the filling of the hub. In both cases, venting conditions have been proved crucial and the importance of vent design in commercial die design was highlighted. 


\section{Table of Contents}

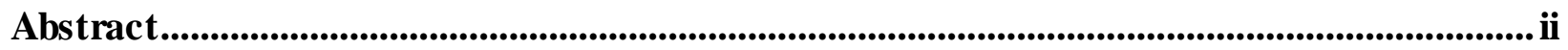

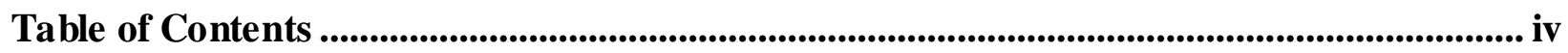

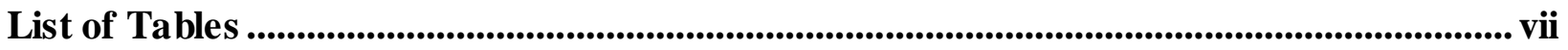

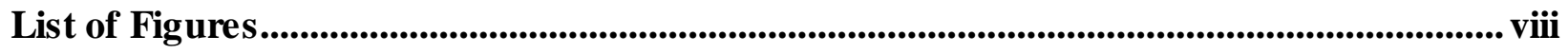

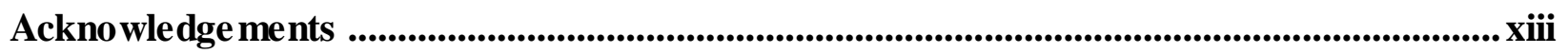

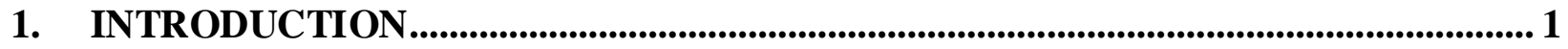

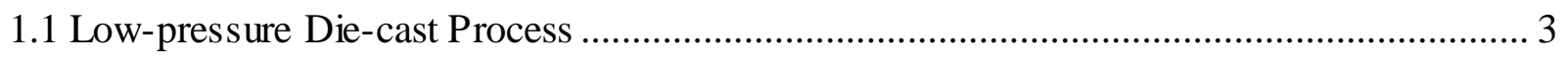

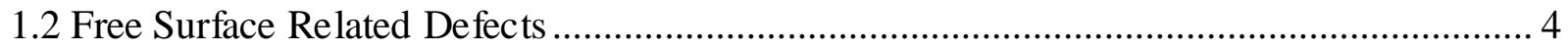

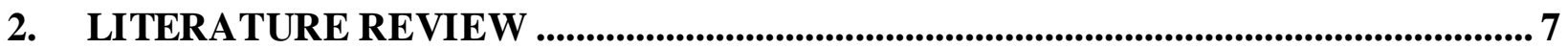

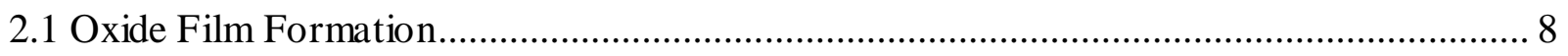

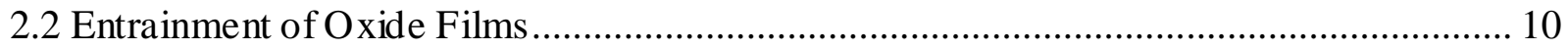

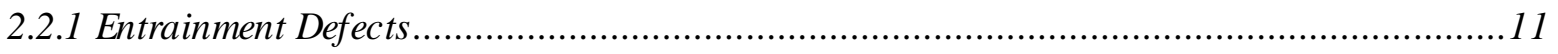

2.2.2 Entrainment Processes ............................................................................... 16

2.3 Computational Modeling of Casting ..................................................................... 27

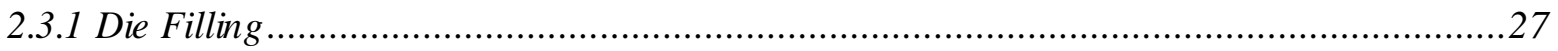

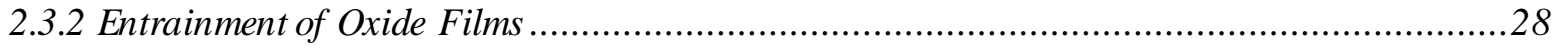

2.4 Water Analogue Technique (Physical Model) .......................................................... 31

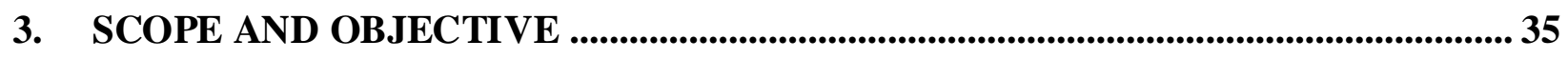

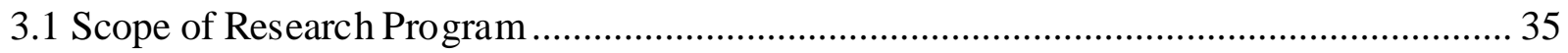

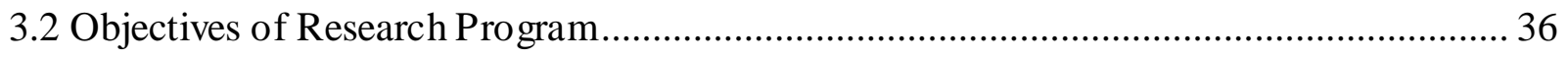

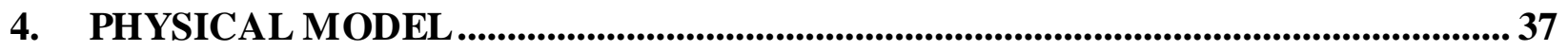




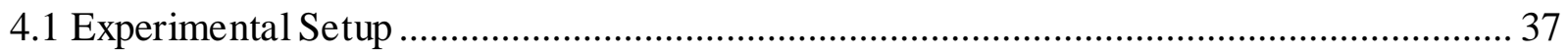

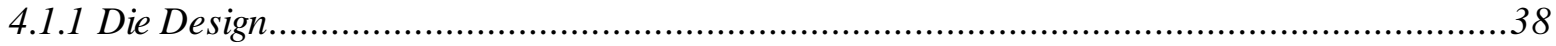

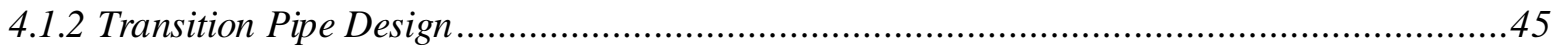

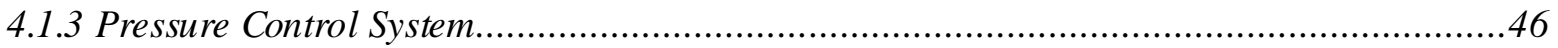

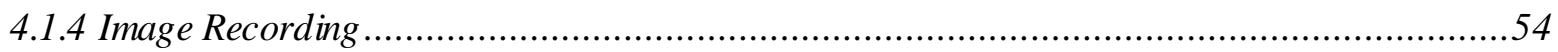

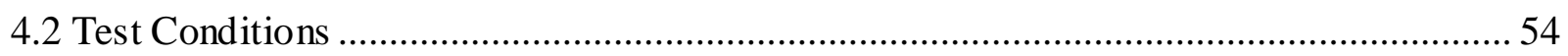

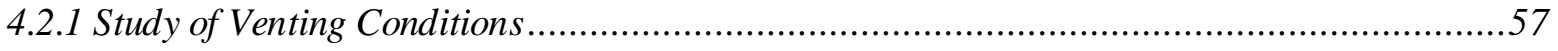

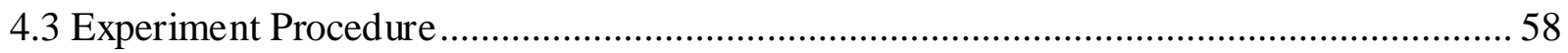

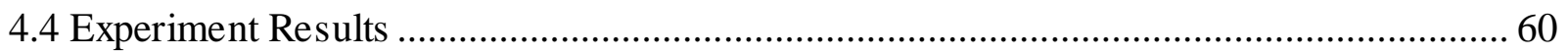

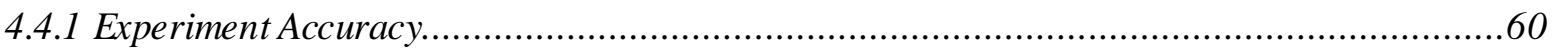

4.4.2 Preliminary Examination to the Die Filling Process .................................................65

5. COMPUTATIONAL MODEL DEVELOPMENT ................................................... 67

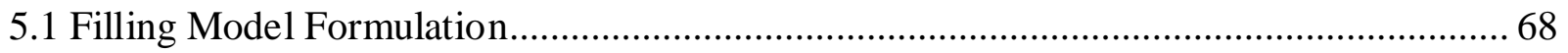

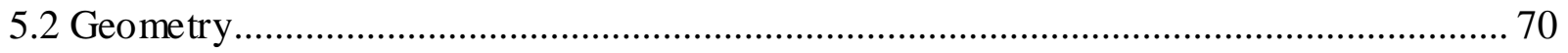

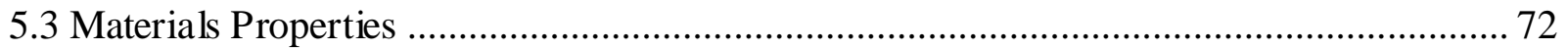

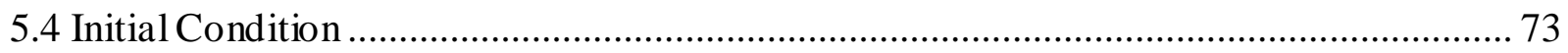

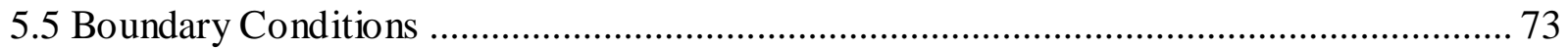

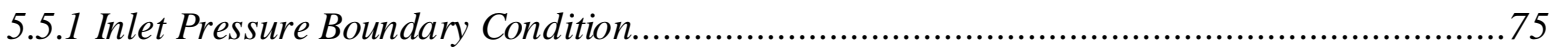

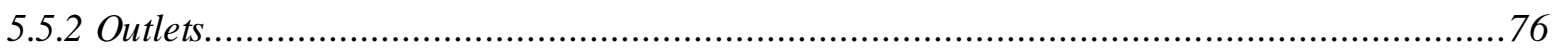

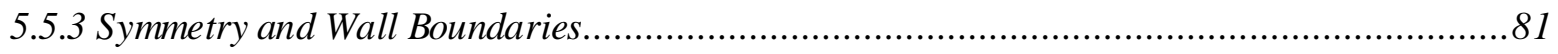

5.6 Verification of the Model................................................................................. 81

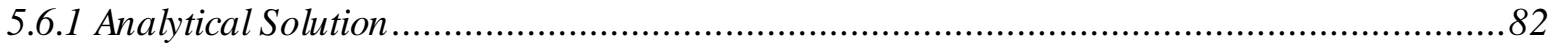

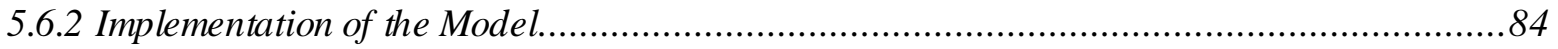

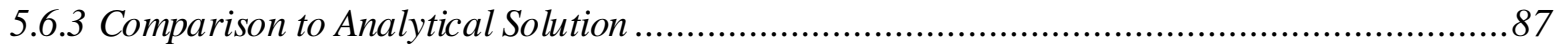

5.7 Preliminary LP Die Computational model Results ................................................... 90 


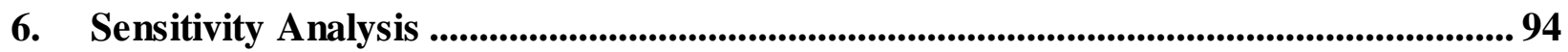

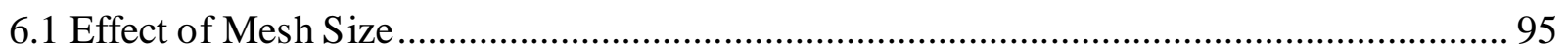

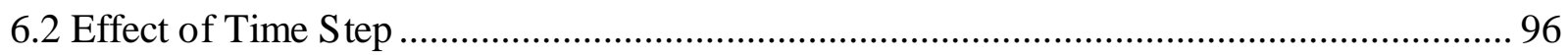

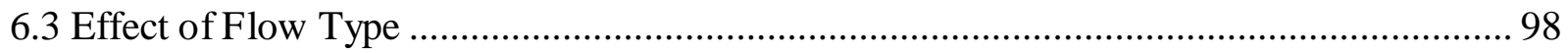

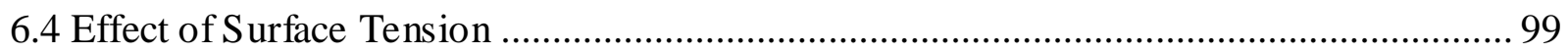

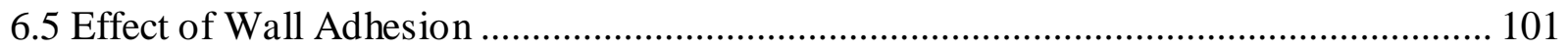

6.6 Effect of Vent Resistance Coefficients ................................................................. 103

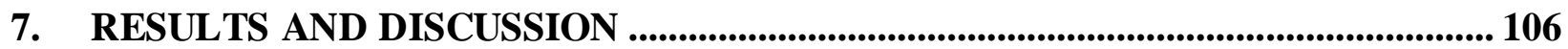

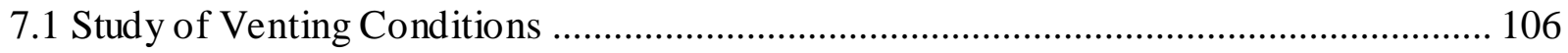

7.1.1 Venting Capacity - Assessment and Preliminary Computational model Validation ............. 107

7.1.2 Critical Phenomena during Filling ................................................................. 109

7.1.3 The Number and Locations of Vents - Final Validation ............................................ 113

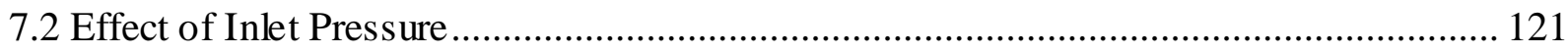

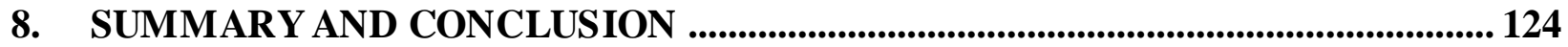

8.1 Recommendations for Future Work ................................................................ 126

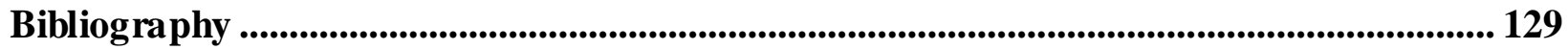




\section{List of Tables}

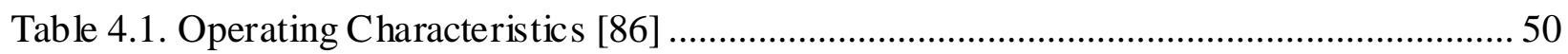

Table 4.2. Specifications of USB - 6009 DAQ board ...................................................... 51

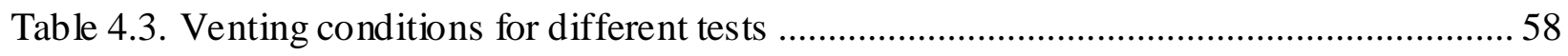

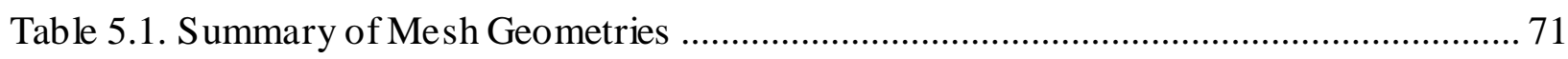

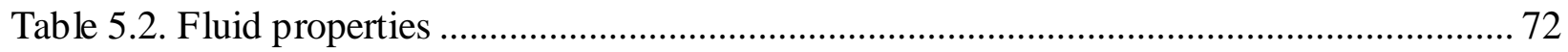

Table 5.3. Pressure curve data applied in the computational model, obtained from water filling experiment. Test \#10 values presented ...................................................................... 75

Table 5.4. Parameters and the corresponding values used in the analytical solution .................. 89

Table 6.1. Summary of the factors investigated in the sensitivity study ............................... 94

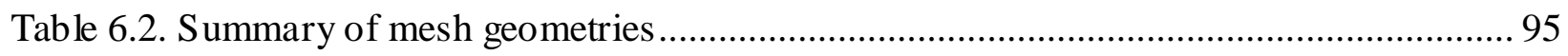

Table 6.3. Vent resistance coefficients for baseline, resistance increased $75 \%$, and resistance

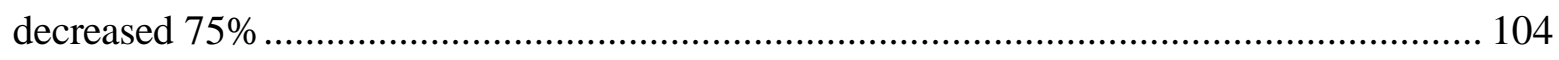

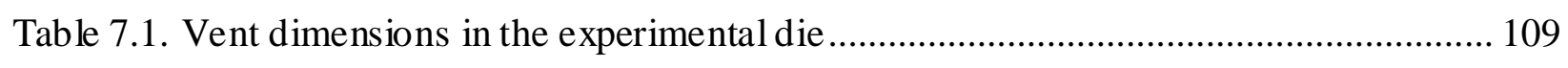

Table 7.2. Vent resistance coefficients for models with different vent combinations ............... 120 


\section{List of Figures}

Figure 1.1. Oxide crack found in the hub area of an as-cast LPDC aluminum alloy wheel.......... 2

Figure 1.2. Oxide fold seen in the hub area of an as-cast LPDC aluminum alloy wheel (courtesy

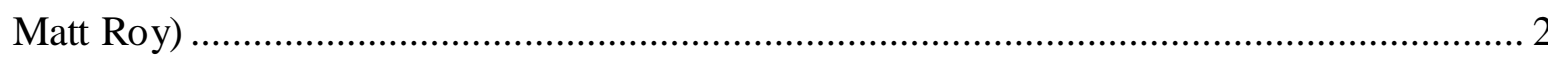

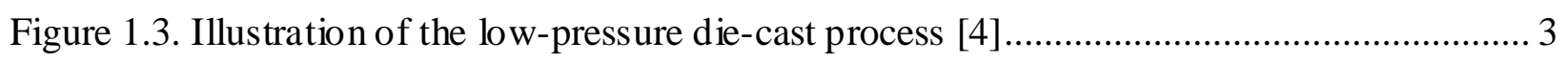

Figure 1.4. Typical press ure curve used in low-pres sure die-case process [4] ......................... 4

Figure 1.5. Drawings illus trate the complexity of an automotive wheel ................................... 5

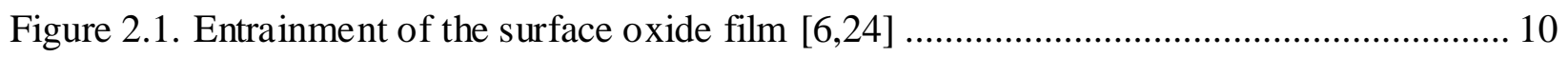

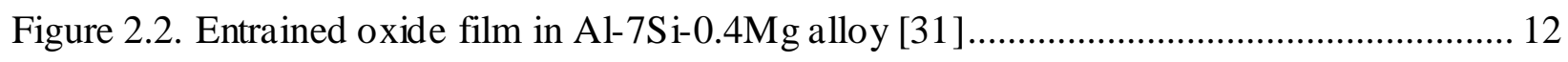

Figure 2.3. Schematic of the experimental setup for adding $\mathrm{TiB}_{2}$ particles to liquid

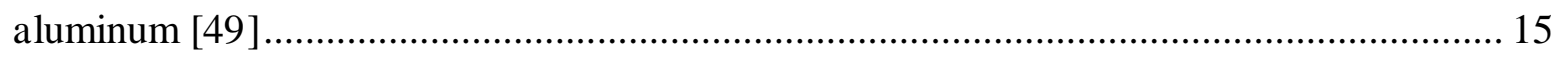

Figure 2.4. Illustration of force balance in the surface of a liquid [23] ................................... 17

Figure 2.5. Bending resistance of castings VS in-gate velocity [50] ................................... 18

Figure 2.6. A pouring stream impinging on the liquid pool, incorporating oxide film and air

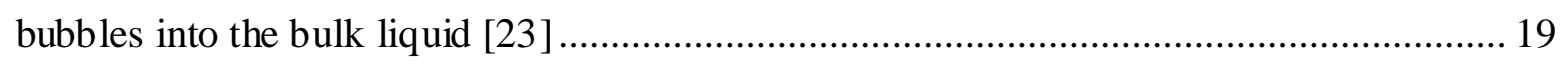

Figure 2.7. Illus tration on treating returning waves in the same manner as a hydraulic

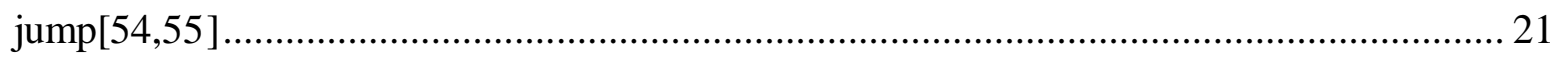

Figure 2.8. X-ray video showing the filling of the runner and die cavity with a returning wave

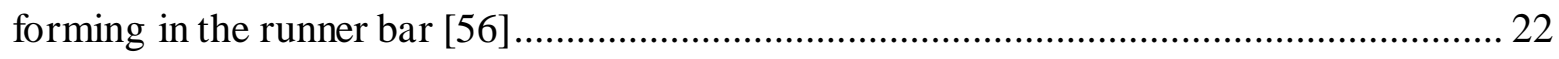

Figure 2.9. Schematic of rising bubbles and cross-sections of the progressive collapse of the

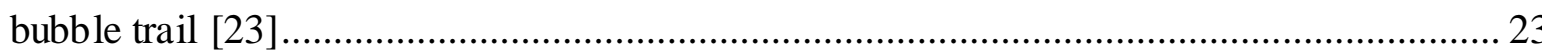

Figure 2.10. Velocity vector plot of aluminum filling a 3D wedge section of a wheel geometry.

Recirculation can be seen in the hub face and hub/spoke regions [60] ............................ 26

Figure 2.11. Surface collision schematic with markers placed at the collision area [51]...........29 
Figure 2.12. Potential surface oxide film entrainment quantified by considering the originated excess free surface area [74].....

Figure 2.13. Comparison between the water solution flowing state and aluminum flowing state at different times [79] 33

Figure 4.1. CAD models of wheels 39

Figure 4.2. Side view of a die-tooling as sembly for typical LPDC process, with a 2D profile of both the die and the sprue highlighted (the right brighter part) 40

Figure 4.3. An extrusion of a simplified wheel profile, with $1 \mathrm{~mm}$ hexahedron grids 41

Figure 4.4. Flow predictions at five locations in the model domain for free-slip wall boundary conditions and no-slip wall boundary conditions with different thicknesses 43

Figure 4.5. A transparent water model made of plexiglas 45

Figure 4.6. Drawings of the transition pipe (courtesy Ross McLeod)..... 46

Figure 4.7. Schematic of the implemented pressure control system. 48

Figure 4.8. The PID control program developed within LabVIEW. A is the setpoint profile, B is the PID controller, $\mathrm{C}$ is the DAQ assistant and D is the signal output interface. 53

Figure 4.9. An example of the pressure curves for A356 and water 56

Figure 4.10. An example of the predefined and real time pressure curves..... 57

Figure 4.11. The calibrated transfer function. 59

Figure 4.12. An example of the repeatability of the water analogue experiment showing four separate tests at five different time periods.(Test \#1) 64

Figure 4.13. Video images showing examples of filling behavior. Each grid square shown in the background is equal to $5 \mathrm{~mm}$ 65

Figure 5.1. 2D mesh of sprue and wheel cavity. $1 \mathrm{~mm}^{3}$ vent can be seen in the upper close up view 
Figure 5.2. Summary of initial condition 73

Figure 5.3. Summary of bound ary conditions 74

Figure 5.4. Schematics of the methodology employed to effectively address venting in CFX.... 78

Figure 5.5. Vent geometry in the die cavity with the outboard flange rim included 79

Figure 5.6. The quadratic relationship between pressure drop and the corresponding averaged vent velocity 80

Figure 5.7. Illustration of the simple case used to evaluate backpressure simulation (courtesy Dr. Jingdong Zhu) 82

Figure 5.8. Quasi 2D mesh of the $1100 \mathrm{~mm} \times 200 \mathrm{~mm}$ model domain used as validation against the analytical solution (courtesy Dr. Jingdong Zhu) 85

Figure 5.9. The filling state at $0.2 \mathrm{~s}$ when the die cavity is $90 \%$ full 88

Figure 5.10. Comparison between numerical and analytical solutions 89

Figure 5.11. Static pressure contour plots for water phase 92

Figure 5.12. Velocity magnitude and direction plots for water phase 93

Figure 6.1. Comparison of free surface height versus fill time for the three models with mesh sizes of $0.5 \mathrm{~mm}, 1 \mathrm{~mm}$ and $2 \mathrm{~mm}$ 96

Figure 6.2. Comparison of free surface height versus fill time for the three models with time step sizes of $0.005 \mathrm{~s}, 0.01 \mathrm{~s}$ and $0.05 \mathrm{~s}$ 97

Figure 6.3. Water volume fraction plots for the baseline model 99

Figure 6.4. Water volume fraction plots for the model that include turbulence 99

Figure 6.5. Comparison of free surface height for $6 \%$ lower surface tension, baseline surface tension and $6 \%$ higher surface tension. 100

Figure 6.6. Water volume fraction plots for the model with surface tension force inactive 101 
Figure 6.7. Water volume fraction plot for the model with surface tension force inactive, showing unreasonable free surface shape at the junction of the outboard flange and the rim at $20 \mathrm{~s}$ 101

Figure 6.8. Comparison of the effect of wall adhesion on the free surface shape below the hub vent (images have been cropped)

Figure 6.9. Water volume fraction plots for the model that include wall wettability effect....... 103

Figure 6.10. Water volume fraction plot for the model with wall wettability model, showing (a) discontinuity in the liquid, and (b) an example of unreasonable flow behavior (images are cropped)

Figure 6.11. Comparison of free surface height versus fill time for the three models with different vent resistance coefficients 104

Figure 7.1. Comparison of the free surface height between computational models with different vent capacities and the physical model. Results of Test \#1 shown. 108

Figure 7.2. Images showing defect entraining filling phenomena seen during the die filling process: 1) bubble in outboard flange, 2) \& 3) bubbles below the free surface, 4) fold-in of the free surface, and 5) potential fold-in of the free surface and bubble entrained below it. The shape of the free surface and bubbles are marked by lines.

Figure 7.3. Free surface plots for the case with vent resistance coefficients lowered by $75 \%$... 112 Figure 7.4. Definition of discrete domains within the wheel profile 114

Figure 7.5. Time taken to fully fill discrete locations of the water model. 114

Figure 7.6. Comparison of the fill pattern between experimental result and the computational result for Test \#1 116

Figure 7.7. Comparison of the fill pattern between experimental result and the computational result for Test \#2 
Figure 7.8. Comparison of the fill pattern between experimental result and the computational result for Test \#3

Figure 7.9. Comparison of the fill pattern between experimental result and the computational result for Test \#4

Figure 7.10. Comparison of the fill pattern between experimental result and the computational result for Test \#5 120

Figure 7.11. Comparison of the free surface height between computational models with different inlet pressure boundary and the physical model, showing the results of Test \#1 122

Figure 7.12. Comparison of static pressure at three points in the wheel domain 123 


\section{Acknowledgements}

I would like to thank my research supervisor, Dr. Daan Maijer, for his encouragement, support, guid ance and enthusiasm, which made the completion of this thesis possible. I would also like to thank Dr. Steve Cockcroft, who was also directly involved in this research project and whose suggestions and insights were always valued.

I am also grateful to Dr. Carl Reilly, Dr. Jingdong Zhu, Dr. Luyao and Dr. Ehsan Khajeh. Their strong theoretical background and rich research experience has enlightened me at times of difficulties. Without their help, this program could have been much less enjoyable.

Many thanks go to the machine shop technicians, particularly Ross Mcleod, who has helped to facilitate the experimental work contained within this thesis. I also gratefully acknowledge Wonsang Kim, the electronic technician, for helping me through my electronic emergencies.

Lastly, I would like to extend my thanks to my family and friends, who has made it possible for me to keep cheerful and positive in all circumstances. 


\section{INTRODUCTION}

The automotive industry is moving toward the use of aluminum alloys wheels over the traditional stamped and welded steel wheels because of their light weight, improved aesthetic appearance and design flexibility [1]. However, defects in automotive aluminum alloy wheels continue to challenge foundry engineers and academics alike. One of the most challenging defects is the entrained oxide film, originally described by Campbell [2]. The entrained oxide film, as its name suggest, refers to the entrainment of portions of the oxide film that form on the liquid free surface due to the oxidization of aluminum exposed to the atmosphere. The occurrence of the entrainment events depends on the flow conditions, especially the free surface flow characteristics.

Figure 1.1 shows an example of a free surface related defect in a LPDC aluminum alloy wheel. The defect is an extensive oxide crack formed in the hub region. Figure 1.2 is another example showing a large oxide film that intersects with the surface of the hub. Oxide films entrained in the liquid during casting are thought to significantly contribute to the formation and severity of many other common casting defects such as cracks and porosity. These in turn lead to cosmetic defects in the paint, rim-leak defects and reduced fatigue performance [3]. Depending on their severity they may necessitate repair or even rejection of the wheel, decreasing manufacturing efficiency. Due to the competitiveness of the wheel manufacturing industry, manufacturers cannot tolerate more than a few percent scrap to remain profitable. Thus, understanding how these defects form and developing techniques to reduce them is the primary motivation behind the research presented in this thesis. 


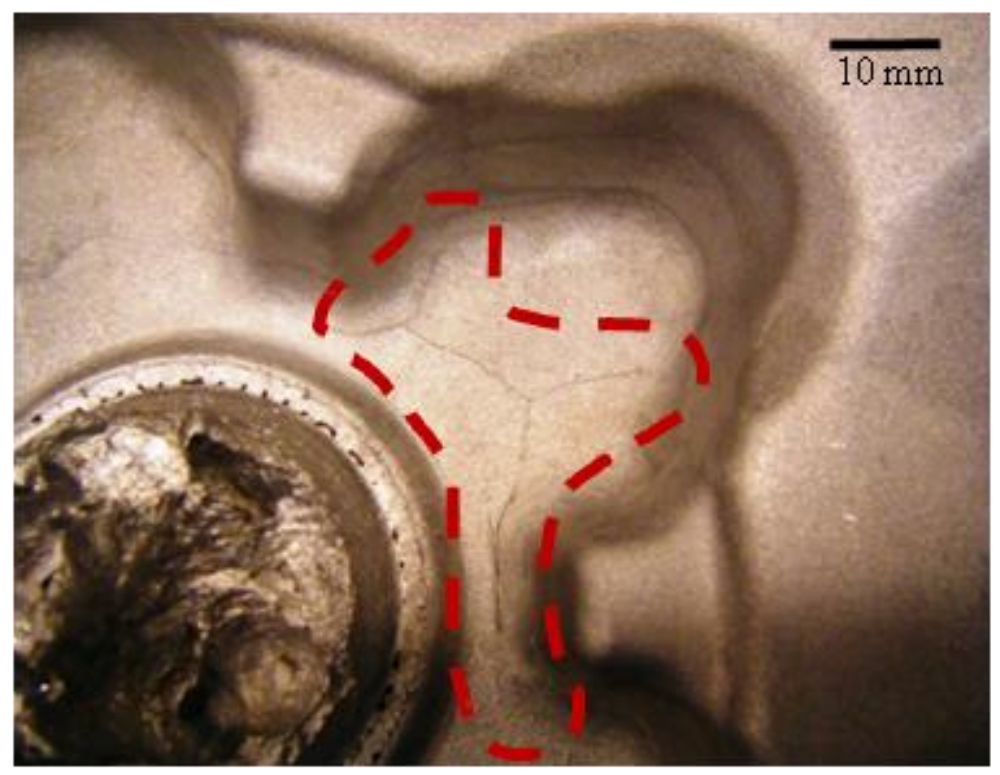

Figure 1.1. Oxide crack found in the hub are of an as -cast LPDC aluminum alloy wheel

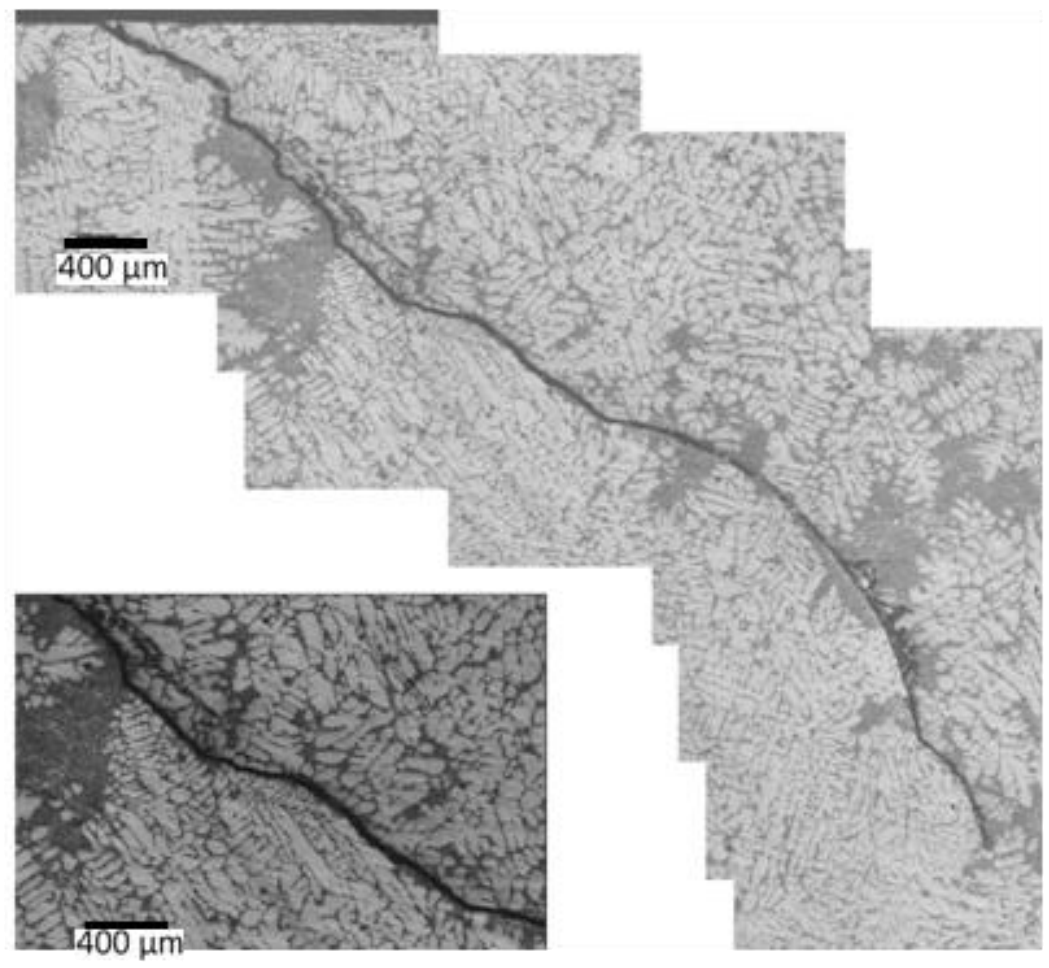

Figure 1.2. Oxide fold seen in the hub area of an as-cast LPDC aluminum alloy wheel (courtesy Matt Roy) 


\subsection{Low-pressure Die-cast Process}

The low-pressure die-cast (LPDC) process is the major manufacturing process used to cast aluminum alloy wheels, owing to its ability to produce geometrically complex parts with good surface finish and at low scrap rates [1,4]. Figure 1.3 shows across-section through a typical LPDC machine. The die assembly is seated above a holding furnace, which contains a reservoir of liquid aluminum. When a casting cycle begins, compressed air enters the holding furnace and pushes the liquid aluminum up to fill the wheel cavity. The air pressure in the holding furnace follows a predefined pressure curve, as shown in Figure 1.4. It contains a filling stage where the pressure is increased to fill the sprue and die cavity, a pressure holding stage where the pressure is held to ensure the cavity is full, and a pressure ramp stage to improve feeding during solidification. After the wheel solidifies, the pressure is released, the die is open and the casting is ejected from the die-casting machine. After a short delay, the die closes, and another casting cycle begins.

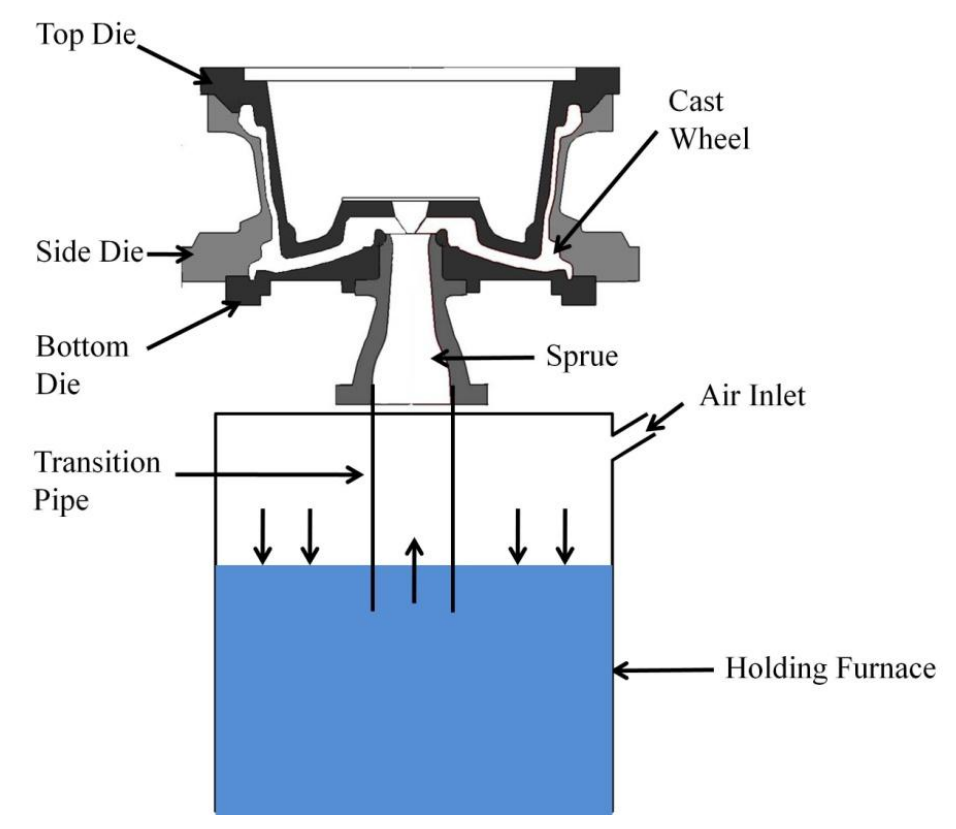

Figure 1.3. Illustration of the low-pressure die-cast process [4] 


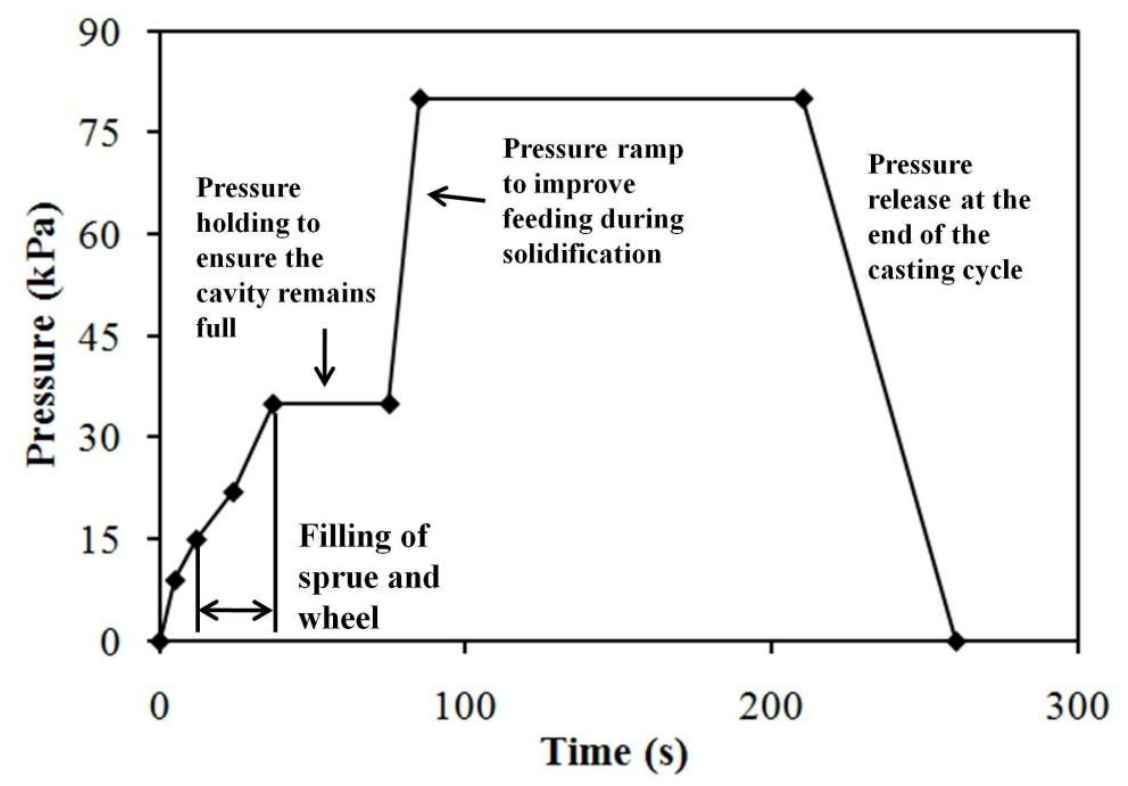

Figure 1.4. Typical pressure curve used in low-pressure die-case process [4]

\subsection{Free Surface Related Defects}

A typical automotive wheel has a very complex geometry. It contains various geometric features including the spoke, rim and bolt-holes, as illustrated by Figure 1.5. These structures can complicate the flow of liquid metal causing convergent free surface flows and turbulence that may potentially lead to the entrainment of oxide films. For example, the windows, which are defined in the industry as the empty spaces between spokes, splits the flow, which then rejoins in the outboard flange and rim. The splitting and rejoining process is potentially harmful because it may lead to the entrainment of the surface oxide film as the two free surface flows converge. 


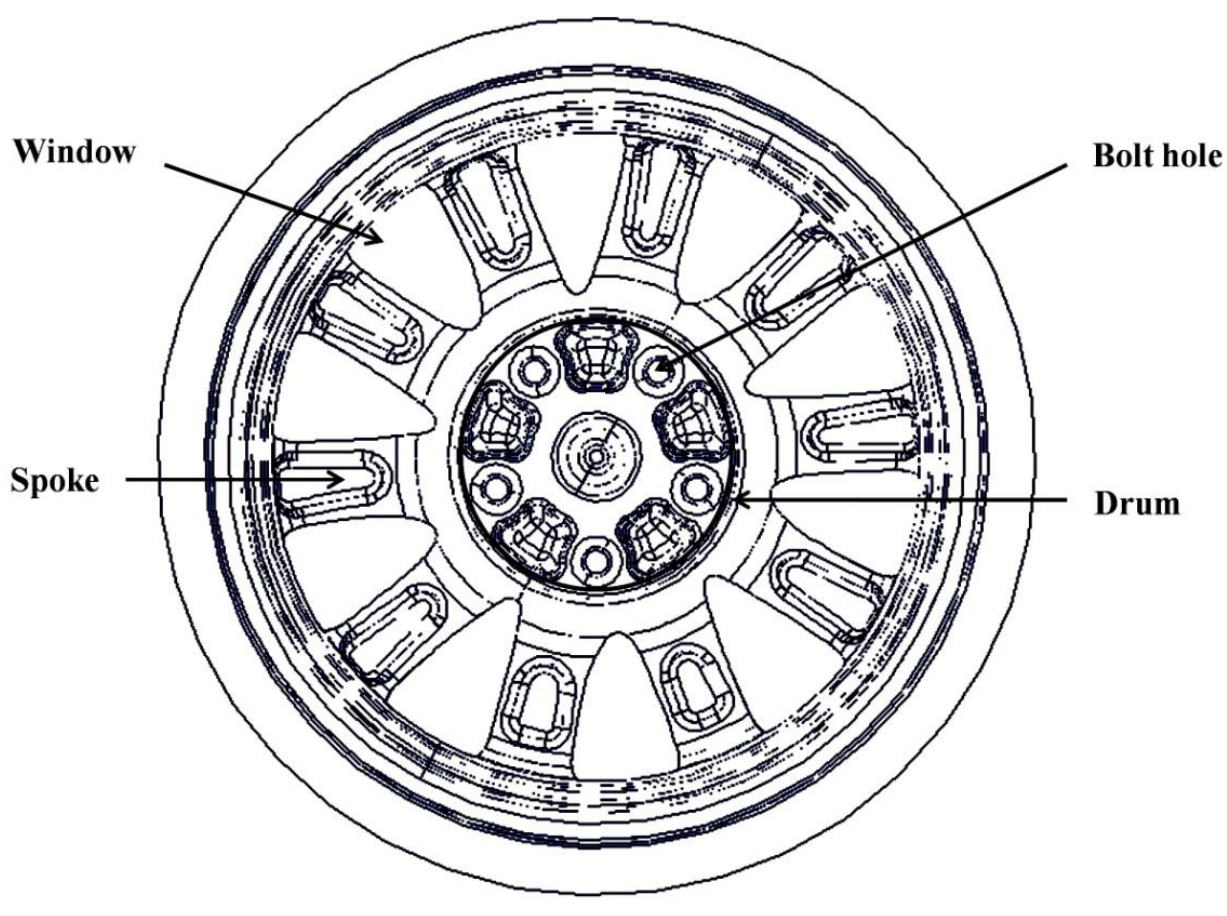

(a) Top vie w of a wheel

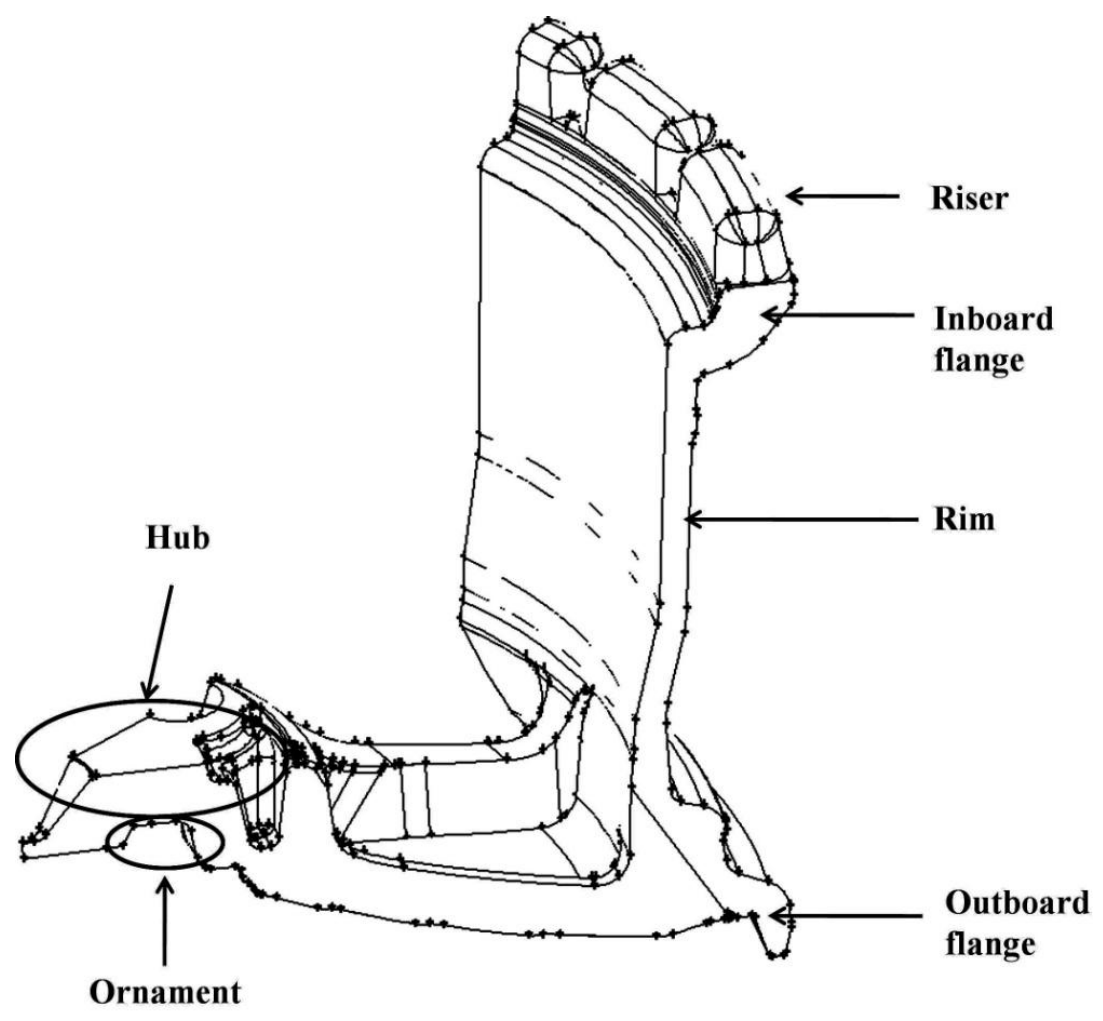

(b) Side vie w of a wheel

Figure 1.5. Drawings illustrate the complexity of an automotive wheel 
The purpose of the research presented here is to improve the understanding of the flow behavior during mould filling associated with the low-pressure (LP) die-cast of aluminum alloy wheels and thereby set the stage for understanding the conditions that lead to the formation of oxide entrainment related entrainment related defects. 


\section{LITERATURE REVIEW}

Aluminum is a reactive and easily oxidized element. The free energy of formation for alumina at $298.15 \mathrm{~K}$ is $-1582.3 \mathrm{~kJ} / \mathrm{mol}$, which is more than ten times of that of $\mathrm{Cu}_{2} \mathrm{O}(-146.0 \mathrm{~kJ} / \mathrm{mol})$ and two times of $\mathrm{Fe}_{2} \mathrm{O}_{3}(-742.2 \mathrm{~kJ} / \mathrm{mol})$ [5]. Therefore the surface of molten aluminum and its alloys is always covered by a solid oxide film in common casting scenarios. The oxide film, while it remains on the surface, protects the melt beneath it from oxidation. When the oxide is incorporated into the bulk liquid, it becomes a submerged film that can lead to various defects which are detrimental to the integrity of castings.

The surface oxide film can be entrained into the bulk liquid in a number of ways, e.g. during charging of the melting furnace, through transfers involving pouring or other types of surface turbulence, and when filling the die $[6,7,8]$. The oxide films entrained prior to die filling are normally thick ones due to the fact that they have sufficient time to grow. These old oxide films can be largely removed by the use of appropriate filters prior to entering the die $[9,10]$. The oxide film entrained due to surface turbulence during die filling may be difficult to remove. Thus it is very important to avoid types of flow that are turbulent during die filling. Due to the opaqueness of the die cavity, the high temperature and reactivity of liquid metal, and the complex geometry of most castings, direct observation of the liquid flow and meaningful characterization of the flow is challenging. Currently, only real time X-ray imaging can be used; however, it is not applicable to all casting scenarios and is cost-prohibitive in most cases. Alternative methods such as computational modeling and water analogue techniques (physical modeling) have been investigated and utilized in both the foundry industry and academia to 
characterize the conditions present during filling of the die, such as fluid velocities and fill profiles. This information has in turn been used for optimization of the casting process $[11,12,13]$

This chapter reviews the information reported in literature that is focused on aluminum oxide films, the defects caused by oxide films, the entrainment processes, computational modeling of die filling and the utilization of the water analogs to study the metal casting.

\subsection{Oxide Film Formation}

The oxidation of aluminum in liquid state is reviewed in this section, with a view to its linkage to defect formation in cast aluminum automotive wheels. For the case of pure aluminum, the film that forms initially on the liquid surface has been shown to be an amorphous form of alumina $[14,15]$. As it thickens, it quickly converts to a crystalline, transition-type alumina, $\gamma$-alumina, which develops within the original amorphous layer [16,17]. It then thickens, and after an incubation period, the $\gamma$-alumina transforms to $\alpha$-alumina. This transformation is accompanied by a $24 \%$ decrease in the volume of the oxide and results in a local build up of stress. This stress is relieved by oxide rupture in weak areas thereby exposing the melt. Molten metal exudes via the ruptured oxide path to the surface where the newly exposed metal almost instantly combines with oxygen to form additional amorphous oxide. This process is called breakaway oxidation. The resulting protruding structure is described as a nodular oxide growth, which is found to be associated with dross formation and metal loss $[17,18]$.

There are several factors that strongly affect the composition, morphology and oxidation kinetics of the surface oxide film in aluminum and its alloys, namely; alloying elements (such as magnesium[6,18,19], strontium[6,14], beryllium[6,14] and sodium[6,14,20]), temperature [21,22], atmospheric composition [14,16,17], and surface preparation and 
pretreatment of melt stock [16]. Studies on oxidation kinetics have demonstrated that the onset of breakaway oxidation occurs sooner in magnesium containing alloys than in pure aluminum. Electron micrographs have shown that alloys with different magnesium contents change the oxide from alumina $\left(\mathrm{Al}_{2} \mathrm{O}_{3}\right)$ to magnesium aluminate crystal / spinel $\left(\mathrm{MgAl}_{2} \mathrm{O}_{4}\right)$ and finally to magnesia (MgO) [6]. Therefore, in the case of A356 (Al-7Si-0.35Mg), which is commonly used in the production of low-pressure die-cast automotive wheels, the oxide film is likely a spinel. The growth of large $\mathrm{MgAl}_{2} \mathrm{O}_{4}$ crystals at the oxide-melt interface generates considerable stress within the oxide film causing the film to fail, leading to breakaway oxidation [18]. Thus it is expected that the oxidation of A356 is faster than that of pure aluminum.

The oxidation kinetics of liquid aluminum have been found to increase with temperature. Additionally, temperature has also been found to influence the morphology of the oxide. By skimming oxide films directly off the melt at different temperatures, Mal'tsev et al. [15] observed that oxide films formed on pure aluminum at temperatures below $680{ }^{\circ} \mathrm{C}$ have an amorphous structure, while crystalline films ( $\gamma$-alumina) were formed between $680{ }^{\circ} \mathrm{C}$ and $690{ }^{\circ} \mathrm{C}$. Similar studies by Impey et al. [16] have found that at $750{ }^{\circ} \mathrm{C}$ oxide crystals ( $\gamma$-alumina) were formed after 5 min and traces of $\alpha$-alumina were found after 5 hrs. Therefore, kinetics would seem to be important.

The typical LPDC cycle from the closing of the die to the ejection of castings takes less than 5 minutes, while the filling stage only takes approximately 21 seconds. Thus the oxide film formed during die filling is likely the amorphous form with localized formation of the magnesium aluminate crystals, though those oxide films that are entrained with pockets of air will keep growing and probably develop into thick crystalline films. 


\subsection{Entrainment of Oxide Films}

The mechanism for entraining a surface oxide film into the bulk liquid is illustrated in Figure 2.1. An unstable flow condition occurs at the liquid surface and causes the surface oxide film to fold upon itself, dry side to dry side, normally entrapping air between the two surfaces. Once entrained, bulk turbulence and fluid flow may transport the entrained film elsewhere in the casting [6,23]. Due to the negligible bonding across the opposed dry interfaces, the folded oxide film resembles and acts as a crack once the melt has solidified.

In the next section the mechanisms by which entrained surface oxide films lead to the formation of defects during solidification, and their degrading effects on castings properties are discussed.
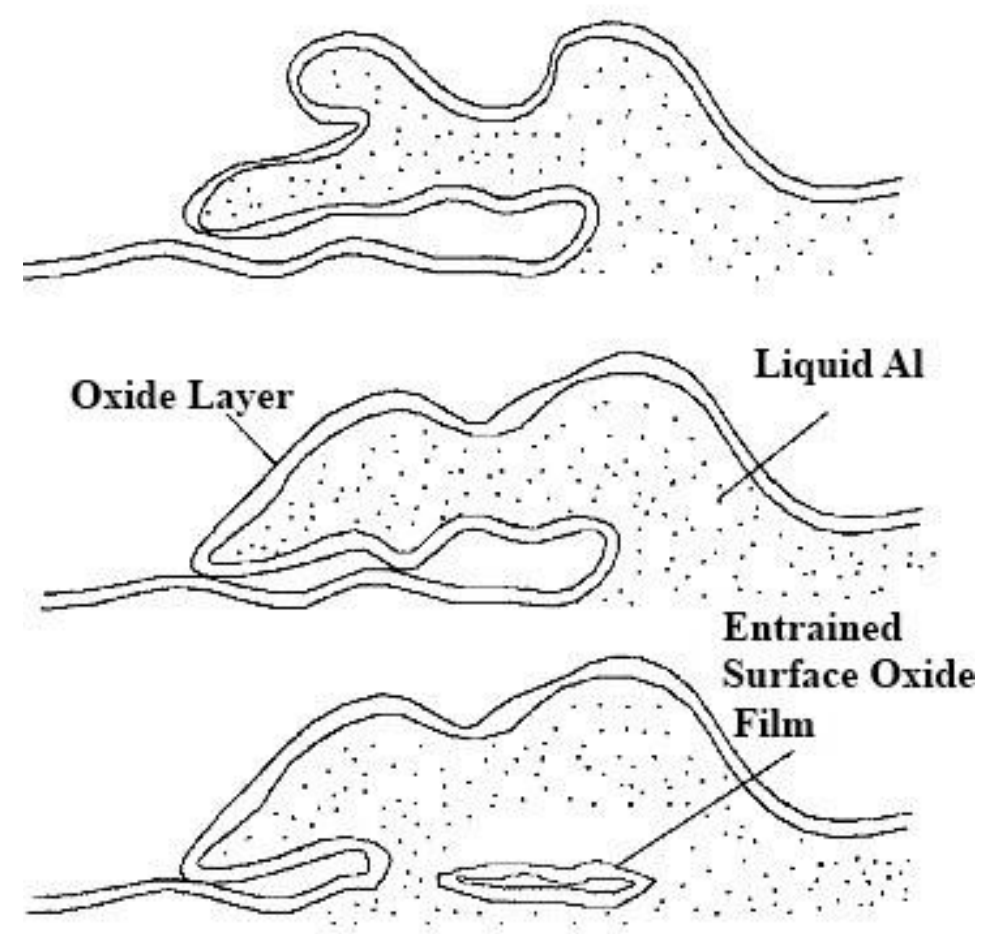

Figure 2.1. Entrainment of the surface oxide film $[6,24]$ 


\subsubsection{Entrainment Defects}

There are three principal defects caused directly by entrainment [23], namely; 1) the entrained surface oxide film in its double film form; 2) the entrainment of air bubbles; and 3) exogenous inclusions. Extensive studies have revealed close associations between these entrained defects with common casting defects such as oxide cracks, porosity, and hot tearing.

A typical entrained oxide film is seen in Figure 2.2 (a). It has an irregular and convoluted form, due to repeatedly undergoing folding caused by excessive surface or internal bulk turbulence, and the thin film's limited rigidity. A high magnification scanning electron microscope image of an oxide is shown in Figure 2.2 (b). This is often referred to as oxide crack [23]. A scale marker was not included with the original image but similar images indicate that the width of the crack is typically below $50 \mu \mathrm{m}$.

In addition to being defects themselves, entrained oxide films are also favored nucleation sites for intermetallic and second phases, and have been reported as the source of decoherence of intermetallic and second phase particles from Al matrices and the internal cracks in second phase particles $[25,26,27]$. The entrained films, when straightened or unfurled, can also constitute large planar barriers that prevent convection during solidification, thus affecting grain size and dendrite arm spacing. These effects in turn have detrimental effects on the tensile strength and fatigue performance of aluminum alloy castings [27]. However, it is worth bearing in mind that the effect of microstructure on mechanical properties of aluminum alloy castings is minor when defects such as oxide films and porosity are present $[28,29,30]$. 


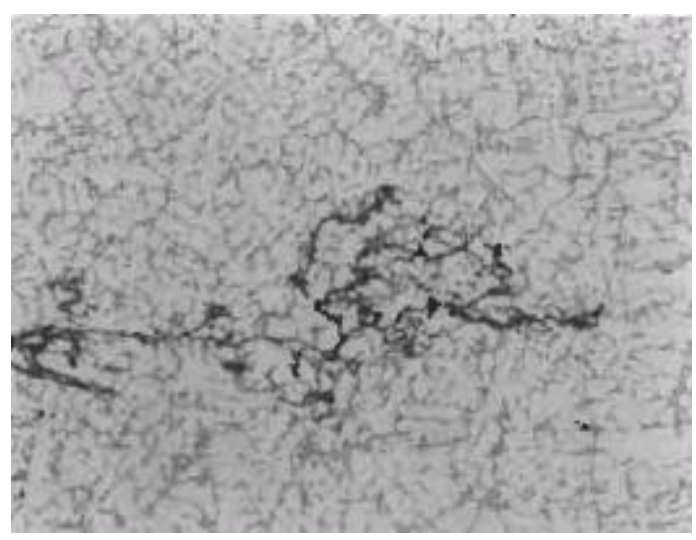

(a) A convoluted structure

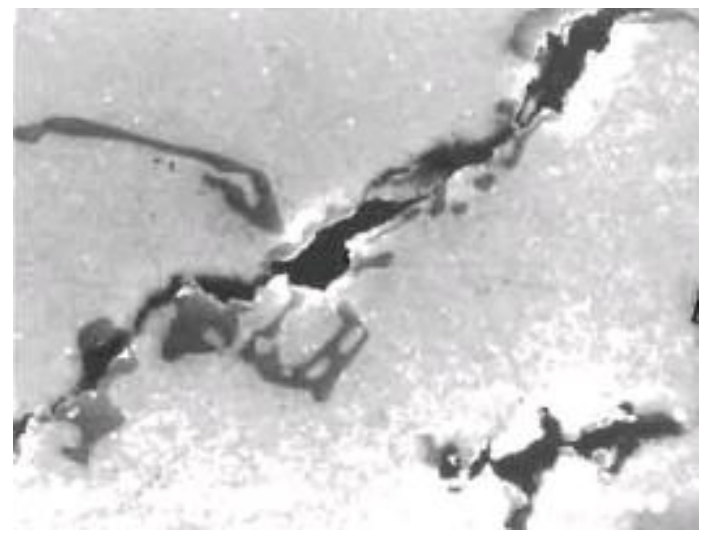

(b) The crack-like feature (close up of a section of image )

Figure 2.2. Entrained oxide film in Al-7Si-0.4Mg alloy [31]

Porosity is one of the most detrimental defects in cast aluminum products. Usually distinguished by the shape and/or scale, pores are characterized as gas-based pores which are round and typically spherical and normally $<\sim 300 \mu \mathrm{m}$ in diameter, and shrinkage pores which are tortuous and can end up in a wide range of sizes. The major driving force in the formation of shrinkage porosity is believed to be inadequate liquid feeding, and in gas-based porosity, gas precipitation due to a reduced solubility of hydrogen upon solidification. In reality, pores are seldom purely gas or shrinkage based as both changing hydrogen solubility and liquid feeding are involved, with their shape determined by the stage of solidification at which they nucleate [32]. Other than the driving force, the nucleation mechanism is the same for the two kinds of pores. Campbell $[2,23]$ postulates that both homogenous and the classical heterogeneous nucleation of porosity in liquid metals is improbable, mainly due to the fact that interfaces in atomic contact are sufficiently strong to be inseparable in any conceivable practical situation, therefore, entrained interfaces must nucleate pores. This statement seems to indicate that all entrained oxide films are associated with pores, and that all pores are initiated by entrained oxide films. However, as described in the beginning of Section 2.2.1, the oxide films can act as a defect in their own 
right and not all entrained films necessarily lead to porosity formation [28]. In addition, pore nucleation is affected by other factors such as thermodynamic and hydrogen supersaturation [33]. Some even proposed that vapor bubbles form spontaneously in a liquid subjected to subatmospheric pressure of considerable magnitude [34].

Another implication that can be drawn from Campbell's statement is that mathematical modeling of porosity would not be accurate if the entrainment of oxide films is not modeled [23]. This again, has been proved questionable. Li and Chang [33] have derived an analytical solution for the mechanism of nucleation and growth of hydrogen pore in the solidifying A356 aluminum alloy without taking into account the flow. Their predicted results of porosity content compares reasonably well with experiments. Lu et al. [35] has successfully modeled the porosity size distribution in A356 tapered cylinder castings, based on the assumptions that hydrogen pores are nucleated heterogeneously, and their nucleation site distribution is a Gaussian function of hydrogen supersaturation in the melt. Again no oxide entrainment process is modeled.

Therefore, a more reasonable description of the effect of entrained oxide film on porosity formation would be that the oxide film promotes porosity formation by facilitating pore nucleation [36]. Entrained oxide films have been repeatedly reported as suitable initiation sites for porosity $[23,37,38]$. X-ray radiography of solidifying castings has been used to observe the formation of shrinkage porosity. In one study, entrained bubbles expanded via the growth of interdentritic porosity as residual liquid is drawn away [23]. Scanning electron microscopy examinations of fracture surfaces and pores show both shrinkage pores and oxide films in close proximity $[39,40]$. Also, dendrites in shrinkage pores have been seen with oxide films draped over them [41,42]. Finally, higher levels of porosity are always found in castings made from 
dirty melts containing high levels of entrained oxides than from clean, filtered melts $[37,43,44,45]$.

Another serious defect that castings can suffer is a hot tear. A hot tear, as the name suggests, is a defect formed at high temperatures, normally above the solidus temperature. The driving force for the formation of a hot tear is the presence of tensile stress acting on a semi-solid structure at high solid fraction. The deformation of the solid can pull the dendrite arms apart at weak regions in the material, such as where there is still liquid present between dendrites in the mushy zone. If there is a lack of feeding in this region, hot tears form $[23,46,47]$. The correlation between hot tears and entrained oxide films has not yet been conclusively demonstrated, though it has been asserted that hot tearing is initiated at entrained oxide films [23]. This notion is a highly empirical one, since it is based largely on successful experiences of eliminating or significantly reducing the occurrence of hot tears in castings by improving the filling system of a casting and thus introducing fewer oxide films.

Exogenous inclusions, such as results from erosion of refractory linings, are another class of defect that are commonly seen in castings. Campbell [23] has pointed out that exogenous inclusions can only be entrained together with the surface oxide film. Campbell has used the findings of a study by Mohanty and Gruzleski's [48] on the mechanisms of heterogeneous nucleation of grains in aluminum castings, to support his theory as they found that $\mathrm{TiB}_{2}$ particles added to liquid aluminum did not nucleate grains, but were pushed to the grain boundaries. Campbell interpreted this result as being due to the oxide film surrounding the particles after being mixed in. This interpretation seems problematic since the $\mathrm{TiB}_{2}$ particles were fluidized and carried directly into the melt through a nozzle placed under the melt surface and filled with inert gas, as shown in Figure 2.3 [49]. It is thus expected that the region of the melt surface where the 
particles were introduced was covered by a negligible amount of oxide film since it was constantly under inert gas protection. Therefore the notion that $\mathrm{all}^{\mathrm{TiB}} 2$ particles added into the melt were covered by oxide films seems unlikely to happen in this reality. An alternative explanation for this phenomenon, as Mohanty and Gruzleski have suggested, is a high interfacial energy between $\mathrm{TiB}_{2}$ crystals and $\alpha-\mathrm{Al}$.

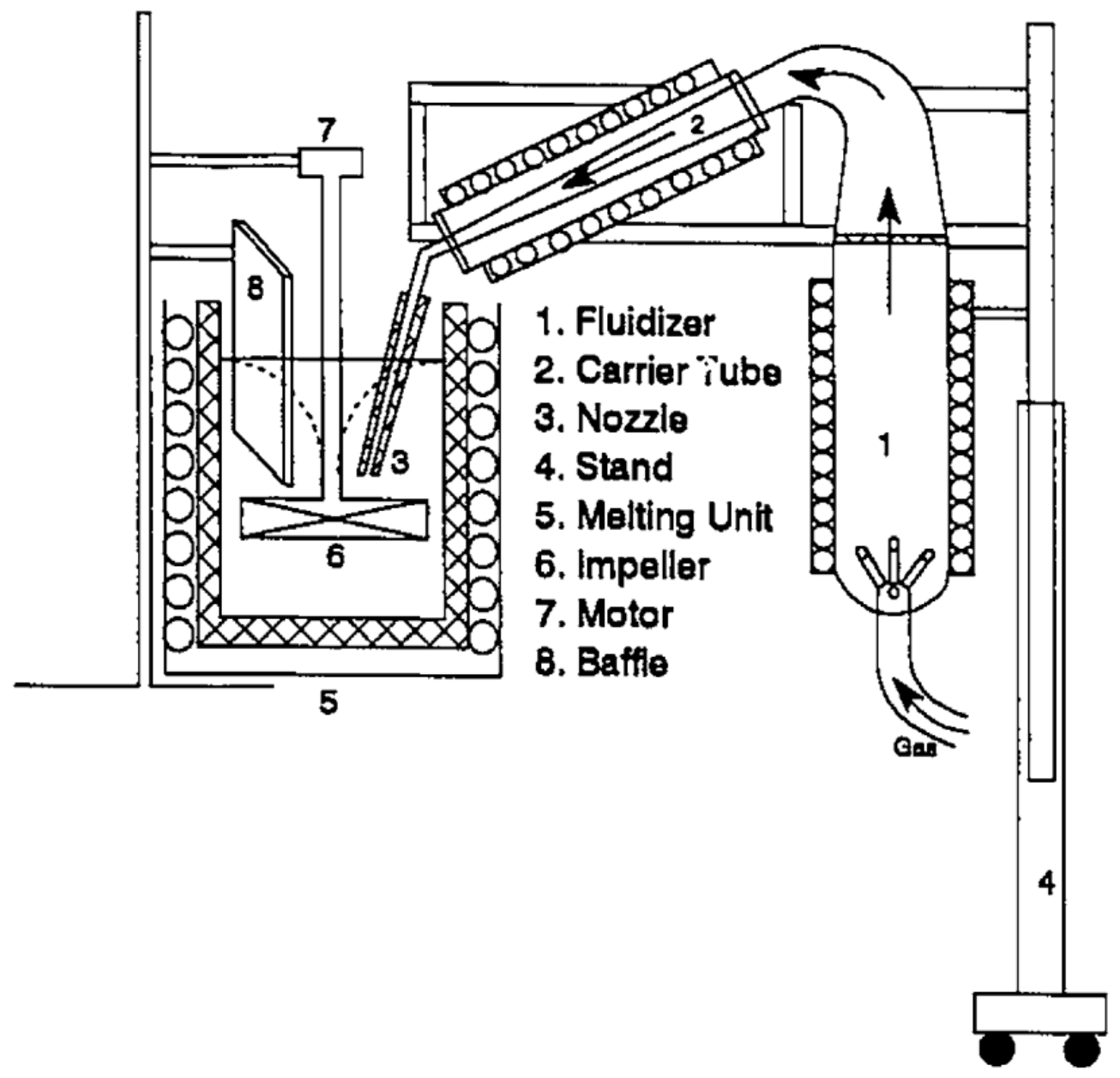

Figure 2.3. Schematic of the experimental setup for adding $\mathbf{T i B}_{2}$ particles to liquid aluminum [49]

Campbell has provided conclusive proof of other exogenous materials, introduced into the melt through a film covered surface, being covered in an oxide film. However, it is the author's belief that there are other ways to incorporate exogenous materials. For example, in the case of die 
casting, the surface layers of the die, i.e. protective oxide films, coatings, and intermetallic compound layers, may be washed away due to the high drag forces of the incoming metal during casting, referred to as erosive wear in die casting applications [4]. Exogenous materials included by this mechanism are not expected to be covered by oxide film.

In summary, many of the defects discussed above, namely oxide folds/cracks, porosity, hot tearing, and exogenous inclusions, appear to be linked in many instances to entrained surface oxide films. Though a variety of factors can come to play during the formation of those defects, the effect of entrained oxide films cannot be ignored.

Industrial experience suggests that the entrainment of surface oxide films is highly dependent on the flow conditions. Extensive studies into the casting of aluminum alloys have identified eight important routes that can introduce surface oxide films into the bulk liquid, as summarized in the following section.

\subsubsection{Entrainment Processes}

\subsubsection{Surface Turbulence}

The term surface turbulence is used to describe free surface instability. It indicates a condition where the local velocity of flow is high enough for the melt to rise above the general level of the liquid surface and subsequently fall back under gravity, potentially causing a fold in a portion of its own surface (Figure 2.4) [23]. Surface turbulence can be as mild as small undulations or as severe as rising jets. 


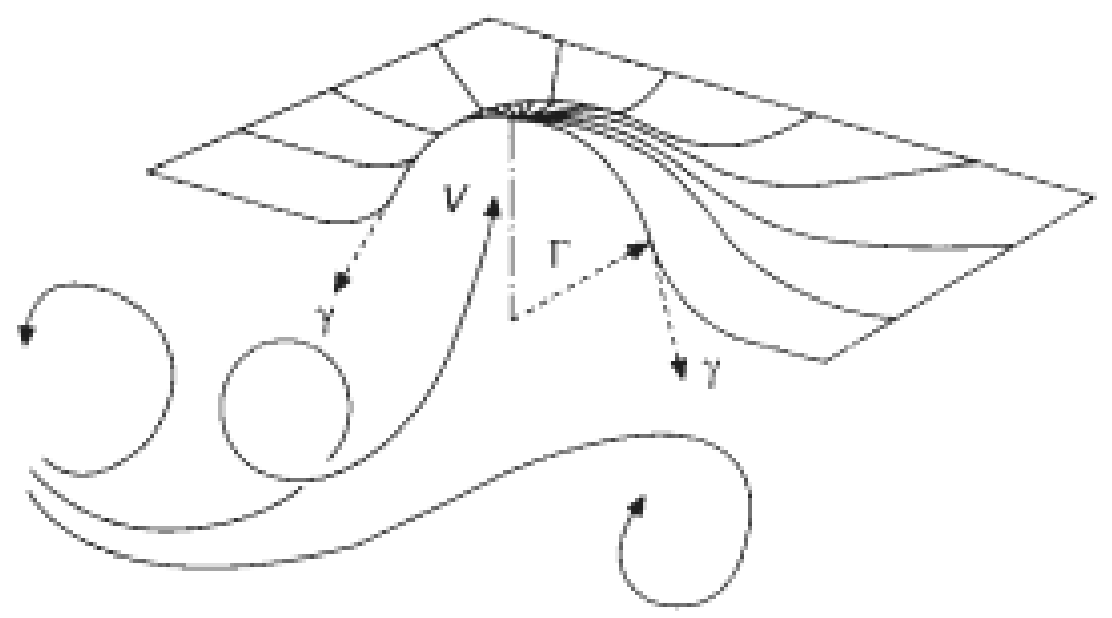

Figure 2.4. Illustration of force balance in the surface of a liquid [23]

By balancing the inertial pressure that acts to perturb the surface with the surface tension that acts to pull the surface flat, a critical velocity $\left(v_{\text {crit }}\right)$ equation can be derived, as given by Equation $2.1[23]$.

$$
v_{\text {crit }}=2 \sqrt[4]{\frac{\gamma g}{\rho}}
$$

where $\gamma$ is the surface tension $\left(\mathrm{N} \cdot \mathrm{m}^{-1}\right), g$ is gravitational acceleration $\left(\mathrm{m}^{\bullet} \mathrm{s}^{-2}\right)$ and $\rho$ is the liquid density $\left(\mathrm{kg}^{\bullet} \mathrm{m}^{-3}\right)$. The critical velocity describes the situation where the local velocity is just sufficient to maintain the two opposing forces in balance. A higher velocity would break the liquid surface and result in surface turbulence. For most engineering metals, the value for the critical velocity is between 0.4 and $0.6 \mathrm{~m}^{\bullet} \mathrm{s}^{-1}[50]$.

Runyoro et al [50] has done some experimental work which has confirmed the calculated critical velocity of $0.5 \mathrm{~m} \bullet \mathrm{s}^{-1}$ for liquid aluminum. The emergence of liquid aluminum at increasing velocities from an in-gate into a casting cavity in a sand die was recorded and used to assess the 
effect of the incoming fluid velocity. Dye penetrant tests and bending tests on the cast plates were conducted to characterize the casting integrity. The results demonstrate that the first folding of the surface film took place at an entry velocity around $0.5 \mathrm{~m} \cdot \mathrm{s}^{-1}$, and for castings with entry velocity higher than $0.5 \mathrm{~m} \cdot \mathrm{s}^{-1}$, there were severe cracks and dramatic decreased fracture stress in bending, as shown in Figure 2.5.

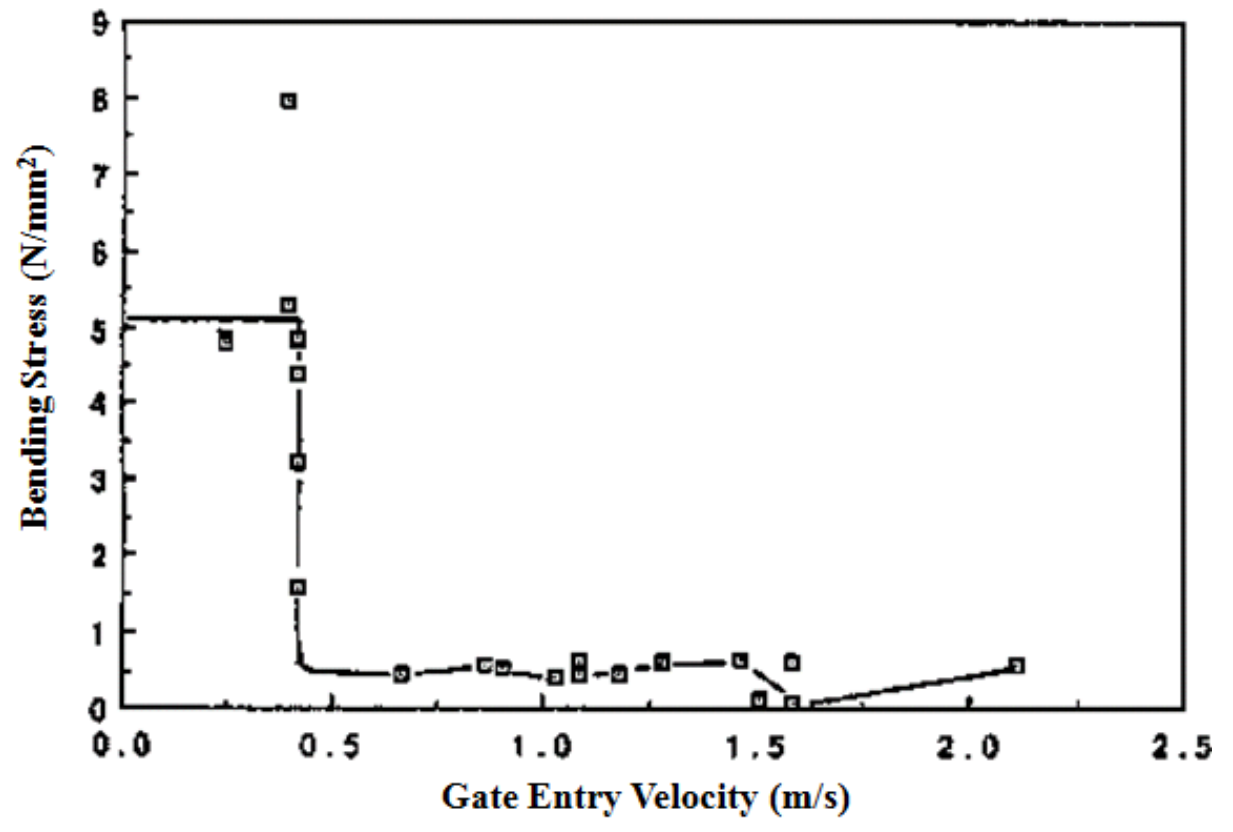

Figure 2.5. Bending resistance of cas tings VS in-gate velocity [50]

\subsubsection{Falling Jet}

Conservation of energy (Equation 2.2) can be used to show that for a given critical velocity, there is a critical height from which a stream of liquid metal can fall under gravity before the surface of the stream is in danger of folding inward. Unfortunately, it is not uncommon for typical casting practice to exceed this critical value. The term, falling jet, is used to describe such conditions. 


$$
\frac{m v^{2}}{2}=m g h
$$

A falling jet commonly occurs during the pouring of liquid metal from a crucible to a basin, in filling the downsprue of a gravity casting, and in dies where there is an edge where the fluid can fall into a deep cavity in the die. There are two mechanisms that contribute to the entraining effect of a falling jet. One is due to the impingement of the falling metal stream on the fluid surface already within the basin or die cavity. This impingement breaks up the surface oxide film and entrains it, sometimes with bubbles, at the point of impingement [51]. Figure 2.6 illustrates this entrainment mechanism. The second entrainment mechanism is linked to the shear force caused by the falling liquid against the inner wall of the surrounding oxide film which is created around the free surface of the falling stream. If the shear stress is high enough, the oxide film tears and accompanies the metal into the die [23].

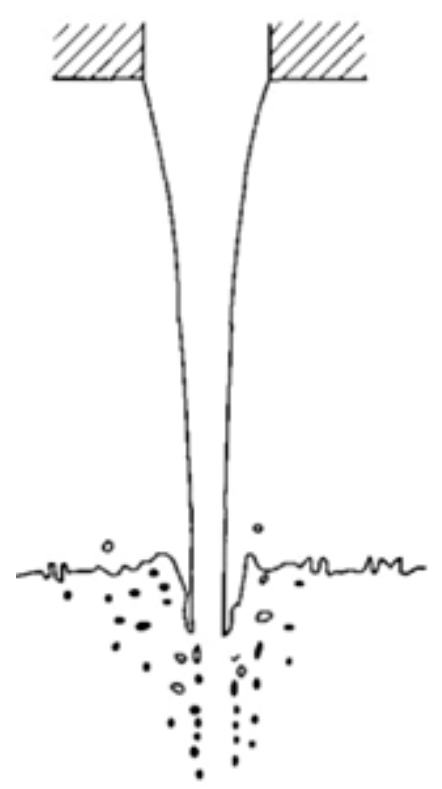

Figure 2.6. A pouring stream impinging on the liquid pool, incorporating oxide film and air bubbles into the bulk liquid [23] 
One experimental study comparing gravity poured castings, tilt poured castings and countergravity filled castings, showed that the most reliable castings were achieved using carefully designed multistage tilt filling cycles in which the free falling of liquid metal was minimized and the metal level was allowed to stabilized during filling [8]. Experience in optimizing the runner systems for investment castings has shown that top filled system produces the most turbulent fill

and the least reliable castings, giving further evidence that oxide films are a major cause of failure [52].

\subsubsection{Hydraulic Jump and Returning Wave}

A hydraulic jump occurs when a flow changes from a sub to a super critical flow regime, i.e. from $\mathrm{Fr}<1$ to $\mathrm{Fr}>1$, where $\mathrm{Fr}$ is the Froude number which is the ratio of gravitational pressure to inertial pressure. A hydraulic jump always manifests itself with a sudden increase in flow height and a reduction in flow velocity. The fluid stream becomes unstable when the Fr number is between 1 and 1.7, and when the Fr number exceeds 1.7, there are waves rolling back, putting the fluid in the danger of folding in its own surface and entraining air bubbles. As the Fr number increases, the flow becomes more turbulent and entraining [53].

However, no classical hydraulic jump, in the form of a standing wave, has been reported in any casting scenario. It is thus concluded that the threshold for a hydraulic jump in liquid aluminum is much higher than that of water and outside the parameters commonly found in the casting process (excluding high pressure die casting). [51]. 
Though hydraulic jumps have not been reported during casting, with manipulation the same theory has been applied to characterize another important flow condition that leads to entrainment - the returning wave (Figure 2.7) [54,55]. Returning waves are commonly formed in conditions where the flow passage has a depth greater than the sessile drop height $(12.5 \mathrm{~mm}$ for liquid aluminum) and the flow channel is not filled. These conditions are commonly found in the runner bars during the transient filling period when the initial stream of fluid impacts the end of the runner bar and then travels back along the runner bar towards the sprue runner junction (Figure 2.8) [56]. This returning wave entrains oxide films and, in severe cases, bubbles at the impingement point.

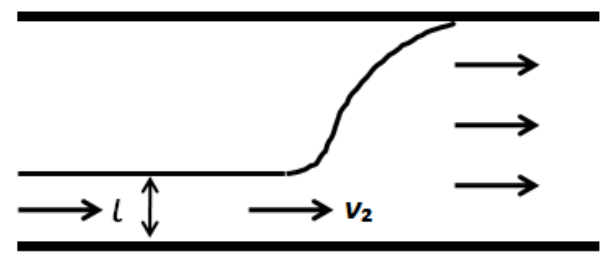

Schematic of classical hydraulic jump:

$$
F r=\frac{v_{1}}{\sqrt{g l}}
$$

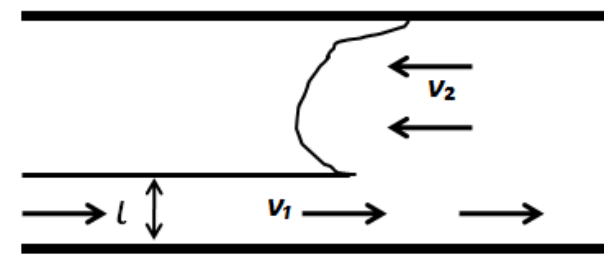

Schematic of returning wave:

$$
F r=\frac{v_{1}-v_{2}}{\sqrt{g l}}
$$

Figure 2.7. Illustration on treating returning waves in the same manner as a hydraulic jump[54,55] 


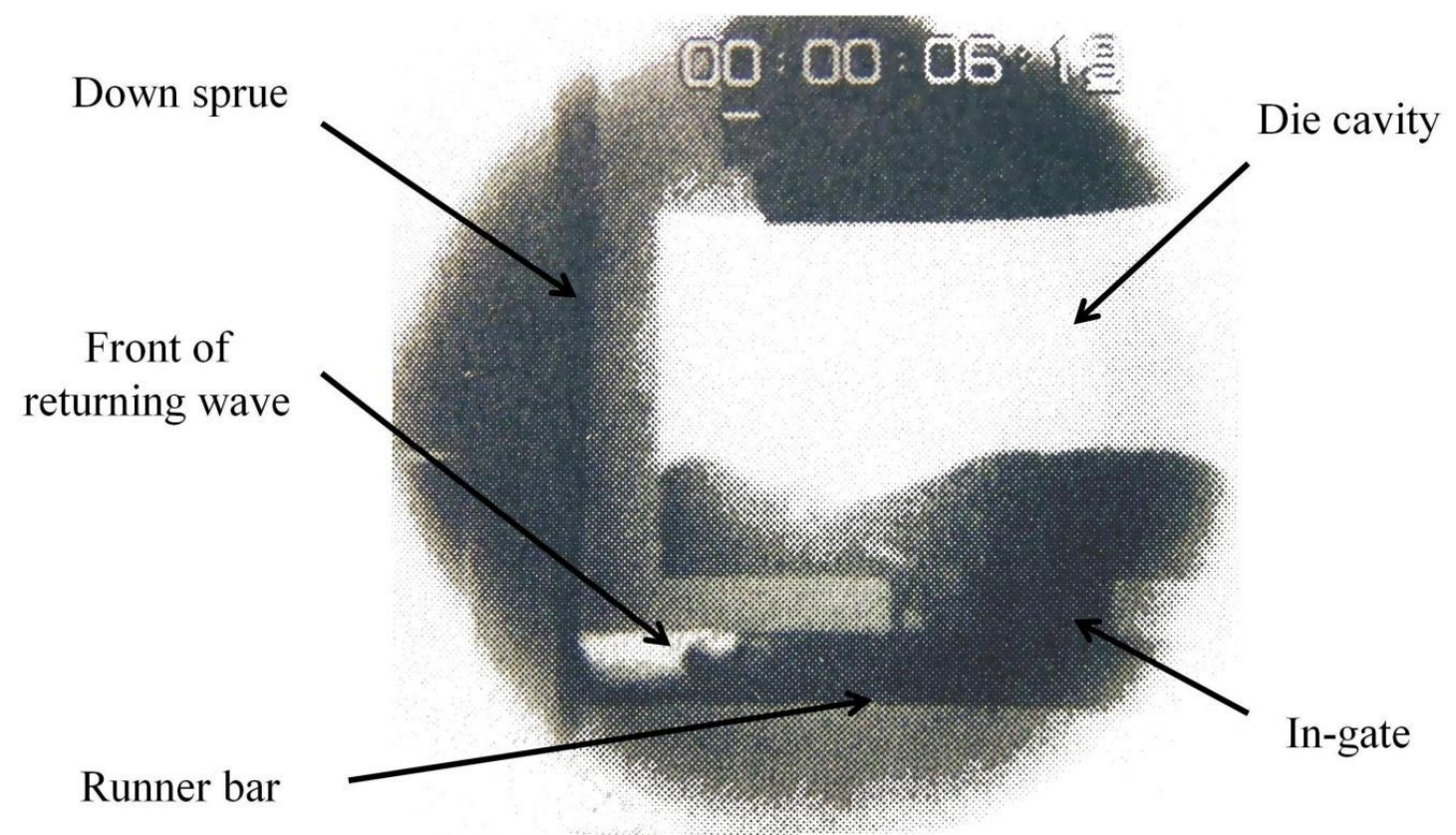

Figure 2.8. X-ray video showing the filling of the runner and die cavity with a returning wave forming in the runner bar [56]

\subsubsection{Oxide Lap, Cold Lap and Fluid Front Collision}

Oxide laps and cold laps are identical defects resulting from slightly different mechanisms. When the melt stops its advance due to reasons such as a break during pouring, the surface oxide film can thicken. When filling resumes, the fresh melt may be unable to break through the thickened surface film, instead it will force its way through a weak point and flood over the old, thick film. Defects formed in this way are normally large asymmetrical, horizontal planar defects, consisting of a lower thick film and an upper thin film [23]. The only distinction between oxide laps and cold laps is that the formation of oxide laps involves no solidification in the liquid front while in a cold lap, the meniscus become partially or completely solidified, thus the name 'cold' laps. 
Fluid front collision is used to describe situations where flow fronts, covered with oxide films, are brought together without successfully joining together or mixing. The result is a large crack between the two surfaces. This often occurs in castings with geometries that require the metal stream to separate and subsequently recombine at some distant location [57].

\subsubsection{Bubble Trails}

When large bubbles are entrained, their buoyancy is so powerful that they rise rapidly through the melt. They do so by splitting the oxide film on the crown, which is almost instantaneously reformed. The old oxide film is left in the bubbles wake as a collapsed tube of oxide (Figure 2.9) [23]. Like other entrained oxide film related defects, the inner surfaces of the oxide tube are dry and therefore unbonded.

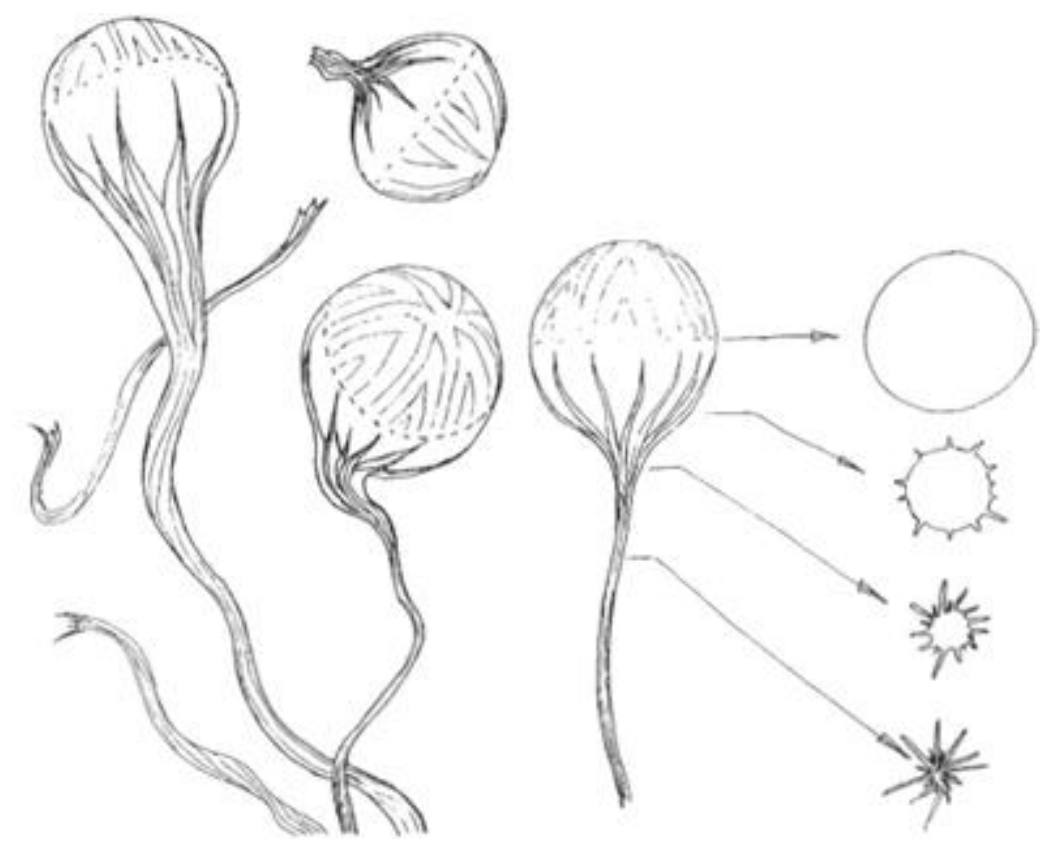

Figure 2.9. Schematic of rising bubbles and cross-sections of the progressive collapse of the bubble trail [23] 


\subsubsection{Other Processes}

For the sake of completeness, other processes [23] which lead to oxide film entrainment are briefly introduced below.

- Melting furnace - When solid charge materials are added directly into the liquid pool of a melting furnace, the oxide film originally on the charge materials becomes submerged. In addition, the charge materials penetrate the melt surface, and bring a portion of surface oxide film originally on the melt into the bulk liquid. Oxide films entrained in this way are normally old and thick films that can be effectively blocked from entering die cavities by filters.

- Microjetting - when the liquid front becomes chaotic on a microscale, and the meniscus of the advancing front may be separated by cracks, through which tiny jets of metal can be ejected ahead of the front. This behavior has been observed in aluminum alloy castings with narrow channels [58].

- Marangoni-driven entrainment - when there are surface tension variations on the liquid surface, there is a potential for Marangoni flow. Marangoni flow results from large gradient in surface tension that may be thermally or compositionally induced. Sometimes the flow can be so intense that it can lead to entrainment, as observed in Chung and Cramb's study [59]. However, Marangoni entrainment is quite unusual and is not expected to happen in most casting processes [23]. 


\subsubsection{Entrainment of Oxide Films in Low Pressure Die Casting of Aluminum Alloy Wheels}

The wheel geometry is such that it requires a change in the flow direction in order to fill, and leads to falling, splitting and rejoining/colliding metal flow fronts. Some of the locations near abrupt geometry changes have been shown to be related to defects such as porosity through computational modeling.

Singh et al [60] developed a computational model of the filling sequence in a LPDC aluminum wheel to study the recirculation of molten metal in the hub face/sprue area and the hub/spoke regions (Figure 2.10). The velocity plot indicates that fluid jetting occurs from the sprue into the hub, and that geometry leads to part of the liquid metal front falling down and recirculating at the hub face. Another recirculation takes place in hub/spoke transition, prior to the region where there is a sudden reduction in cross-sectional area. This recirculating flow pattern was linked to the high porosity found in the hub region. The model was also used to show that by reducing the inlet velocity, the recirculation problems on the hub face could be eliminated. Alternative counter measures that have been reported are to redesign the sprue/hub area [3], or to modify the filling curves [61] to avoid turbulence, and to employ filters at the junction of the sprue and hub

to locally decrease the flow velocity, as filters has been proved effective in increasing the reliability of the cast alloy primarily by controlling the metal velocity [62]. 


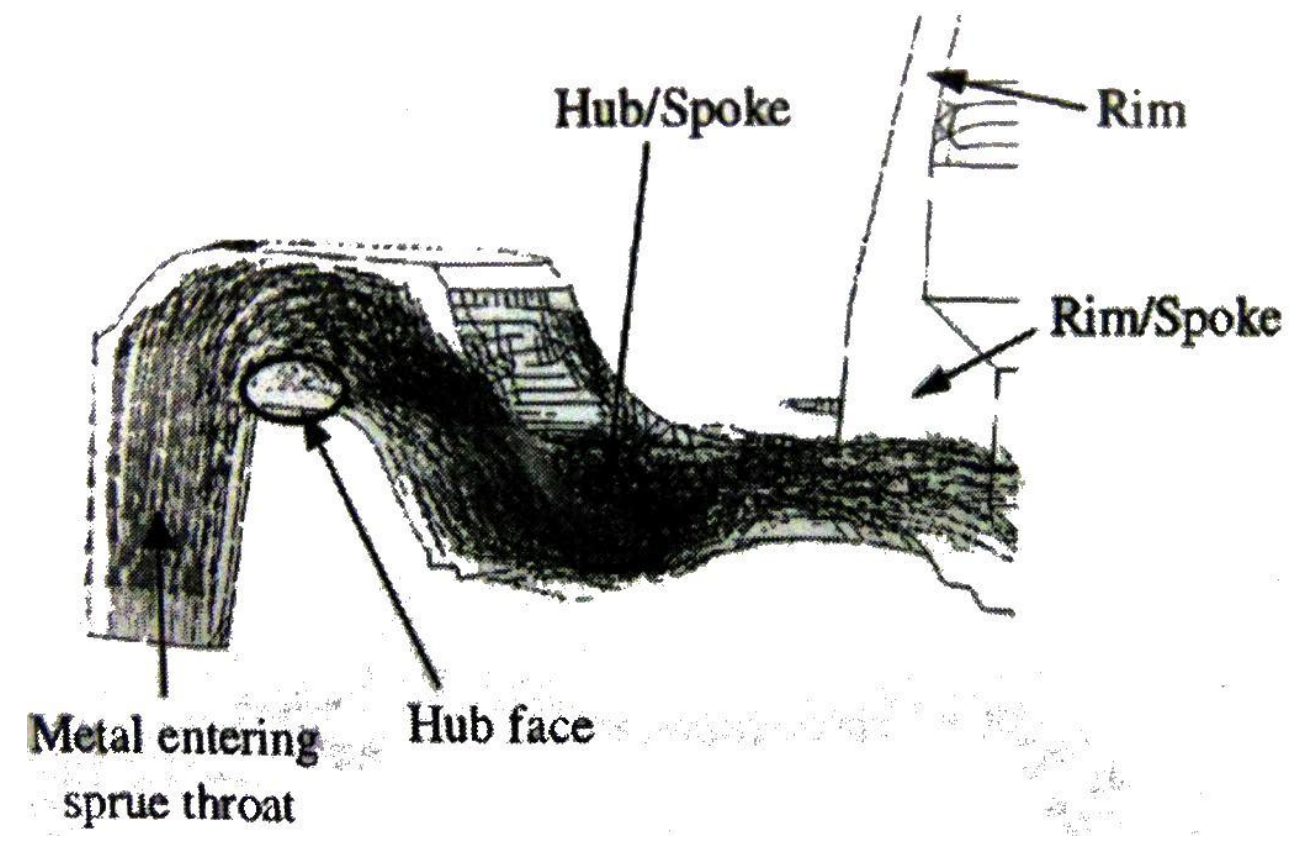

Figure 2.10. Velocity vector plot of aluminum filling a 3D wedge section of a wheel geometry. Recirculation can be seen in the hub face and hub/spoke regions [60]

Another numerical study on the filling and solidification of aluminum alloy wheel has clearly shown gas entrapment at the junction of the rim and spoke where the fluid splits and turns to fill the rim. The authors suggest that the integrity of the casting can be improved if this phenomenon is avoided [63].

One of the critical areas in a cast wheel is the rim where the presence of oxide inclusions and solidification cavities can lead to pressure loss in the wheel / tire unit [61]. Meurer et al. has conducted both laminar fill and turbulent fill simulations of a wheel casting process by applying two different filling curves. The results indicate that in the turbulent fill simulation, the fluid front in the rim has short-range instabilities. This effect was expected to lead to the entrainment of oxides and an increase in porosity in the rim area [61]. 


\subsection{Computational Modeling of Casting}

To reduce cost, the foundry industry is now relying greatly on the use of computational fluid flow and solidification software for product design and process optimization over the traditional trial-and-error methodology. Current academic and industrial research related to the numerical modeling of casting can be roughly divided into five categories: thermal simulations, mould filling simulations, casting defect simulation, solidification structure simulation and stress or deformation simulation [64]. Generally one or more of these areas are simulated for a given casting process, dependent on the objectives of the analysis. Based on the previous discussion of oxide entrainment mechanism, this section has been limited to a review of die filling simulation and the simulation of defects related to entrainment.

\subsubsection{Die Filling}

The awareness of the importance of the filling stage on the final integrity of castings continues to increase. With this awareness has come a need for improved solidification models that are coupled to filling models. There are studies that simulate both the fluid flow and heat transfer [65,66] during filling, whereas others have only focused on the fluid flow [60]. As Campbell has pointed out:

“... the main problems with castings are not those associated with solidification. Approximately $80-90 \%$ of casting problems are associated with the poor running system, i.e. the filling technique of castings." [67]

The accuracy of the die filling simulation has been shown to be sensitive to the surface tension of the liquid and the techniques used to describe the flow of the gas phase in the die cavity [68]. 
The strength of the solid oxide film covering molten aluminum can be treated as an effective surface tension force, which is much larger than that of molten aluminum surface free of an oxide film. The effect of this film is clearly shown by Ohanaka et al [69]. In their experiment, a vertical plate mold was bottom- filled with water, mercury and molten aluminum $(99.7 \% \mathrm{Al})$. The mold for water and mercury was made by acrylic acid resin plate. The front and back plates of the mold for molten Al were made by a heat resistant glass and a steel respectively. The aluminum flow was observed to sway horizontally while surface shapes for water and mercury were relatively symmetric. This observation was interpreted as evidence that the surface oxide was resisting being deformed. The free surface shapes of the initial horizontal streams were different between water, mercury and molten aluminum because of the different surface tension and wetting behavior between the liquid s and the die wall. This experiment highlights the need to take into account the surface tension in developing accurate and detailed models of fluid flow in the die. Another interesting finding relates to the back pressure generated during die filling and how it affects the filling. With the smaller permeability in sand moulds or smaller vents in general, the filling is delayed, which can be a severe problem in casting thin-walled components $[66,70]$. Whether back pressure influences the flow behavior or not depends on the level to which the pressure builds up. In some cases, it was found to have only a minor effect on flow pattern and therefore, did not affect the gas or slag entrapment [66]. However, in other studies, the back pressure in the die becomes so large that it actually forces the air in the cavity to escape through the gating system, which can stop filling while the bubbles escape [71]

\subsubsection{Entrainment of Oxide Films}

There are direct and indirect approaches applied to model oxide film entrainment. Direct simulation of the entrainment event necessarily features the folding action of the liquid surface. 
This is done by assuming that the surface oxide is entrapped when the free surface collides with itself and markers are placed at the area of collision [72,73] (Figure $2.11[51]$ ).

\section{Gas Phase}

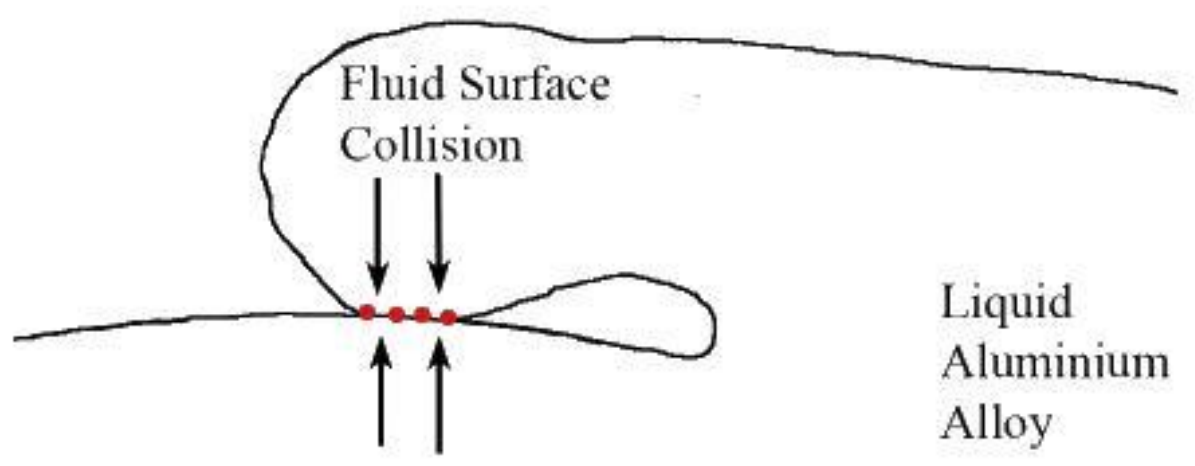

Figure 2.11. Surface collision schematic with markers placed at the collision area [51]

There are a number of different ways to deal with the entrainment of oxide films indirectly, namely, oxide scalar tracking, excess free surface area assessment, and dimensionless number calculations such as Webber number and Froude number.

Oxide scalar tracking assumes that oxide films form at the surface of the liquid metal and accumulate at a constant rate that is quantified by a scalar parameter. The "films" accumulated at the surface are allowed to diffuse into the bulk liquid and advect with the flow of the bulk liquid.

The technique to assess the excess free surface area is based on the volume of fluid (VOF) method. In the VOF method, the liquid volume fraction in each cell is calculated, which allows the determination of the total free surface area. Lai et al. [74] has defined the excess free surface area as the free surface area of the liquid metal, which is excess of that area that the liquid metal would have if it filled the die cavity in a perfectly tranquil way (Figure 2.12). The excess free surface area was used as a measure of the potential for entrainment of the surface and a measure 
in the reduction in liquid metal quality. Through this method, Lai et al. have found that the filling of the crucible and the pouring basin was potentially much more entraining than the filling of castings, suggesting that a poor method for transferring liquid metal could have a significantly detrimental influence on the melt quality. The excess free surface area technique is a straight forward, easy to implement method; however, it does not allow the assessment of where oxides will accumulate in castings. In addition, applying this method to a more complex casting geometry rather than the vertical plate die will have some difficulties, since a loss in the free surface area doesn't necessarily mean this portion of oxide film is entrained, it can also be that the oxide film is pushed against the die wall.

Dimensionless numbers have also been explored to predict the severity of oxide film entrainment. Cuesta et al. have found that the amount of oxide entrained into the liquid is related to the We number [75]. Reilly et al have used the calculated Froude number entrainment criterion to assess the overall quality of the running system, and their predictive results correlated well against quantitative data (Weibul moduli) obtained by experiments assessing three different running systems [54]. 


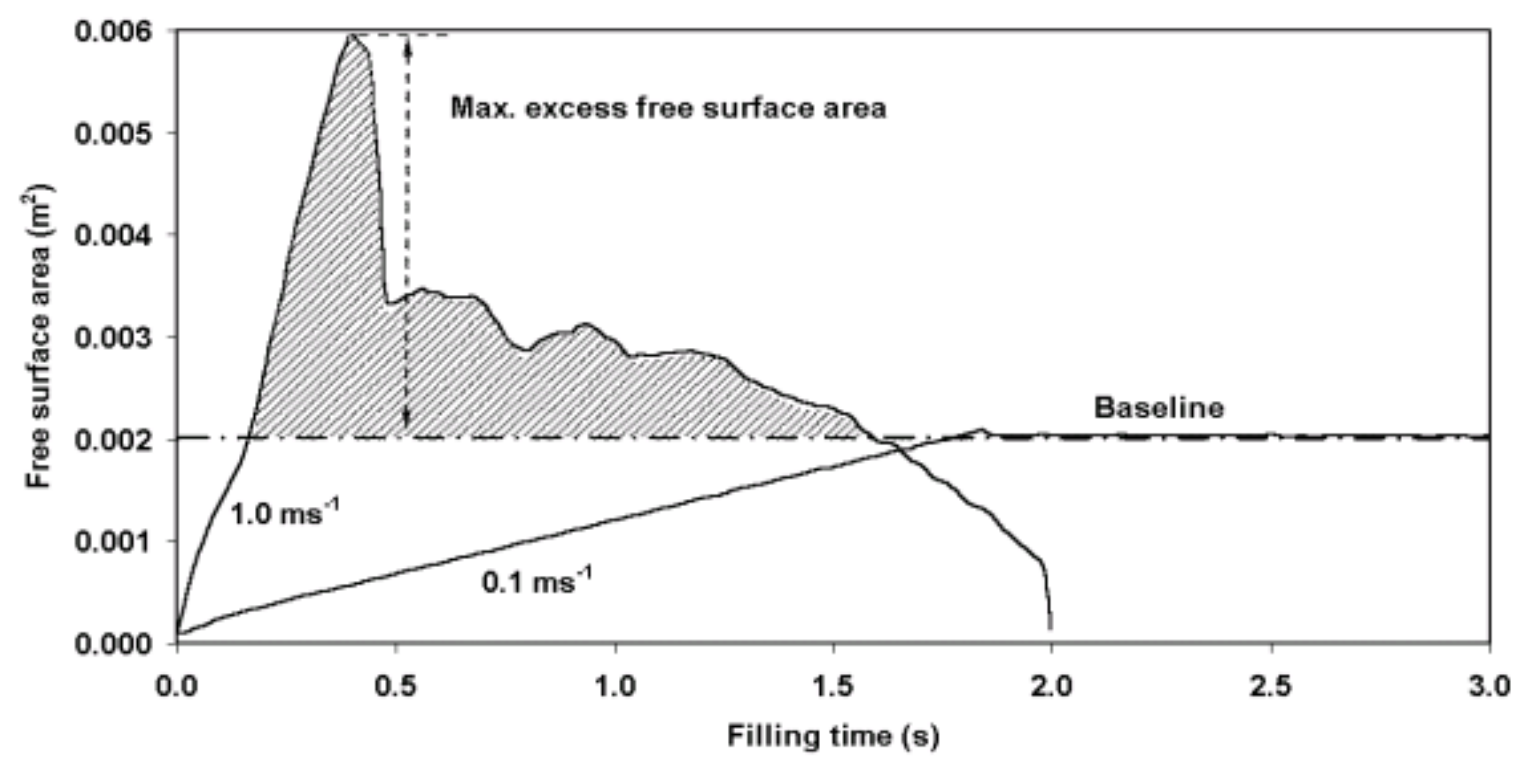

Figure 2.12. Potential surface oxide film entrainment quantified by considering the originated excess free surface area [74]

\subsection{Water Analogue Technique (Physical Model)}

Water analogue experiments in the area of casting refer to the use of water to fill a transparent die to simulate the flow of liquid metal. Depending on the specific purpose and requirements of the experiment, the working fluid can be a mixture of water and another viscous fluid such as polyacrylamide or glycerin, or even tomato paste (this is to mimic the flow of semi-solid melts). Usually a transparent replica of the die is manufactured from a material such as glass or plexiglas to allow direct observation of the liquid flow. The benefits of employing the water analogue technique are that it overcomes the difficulties associated with experimenting with molten metal in a laboratory setting and allows direct observation of the flow pattern [76]. This methodology has been tested in various studies and proved to be valuable and instructive in predicting the movement of metal flow and entrapment phenomena, optimizing casting systems, and validating 
mathematical models. However, the water analogue technique is only suitable for solving fluid flow aspects and not those related to heat transport and material properties [76].

Nguyen and Carrig [76] applied this method to study the gravity tilt casting of copper alloy components. Their purpose was to understand how molten brasses would behave in a number of runner and gating systems, and under different operation parameters. Dynamic similitude was achieved by matching Fr and Re numbers. Conclusions regarding the accuracy of the water simulation for describing gravity tilt casting of molten brasses cannot be made because there was no data for validation. Similar studies can be found in the work of Fortẻ et al [77] in which they visualized the flow of Newtonian and non-Newtonian fluids using water and tomato paste respectively under conditions representing high pressure die casting.

Validation by comparison to liquid metal filling data, though not easily obtainable, is desired if the primary aim of the study is to mimic metal flow through water simulation. Theoretically, in order to achieve a total analogy between the water flow and aluminum flow, the viscosity of water should be decreased by a factor about 2 [78]. However, Zhao et al [79] achieved good correlation between water and the aluminum flow (Figure 2.13) by increasing the viscosity of the water with polyacrylamide to $6.537 \times 10^{-3} \mathrm{~Pa} \bullet \mathrm{s}$, which represents a factor of six increase in viscosity relative to water at $20{ }^{\circ} \mathrm{C}$. The viscosity increase is probably due to that the aluminum melts in the study has already become mushy thus has a larger viscosity than liquid aluminum. 


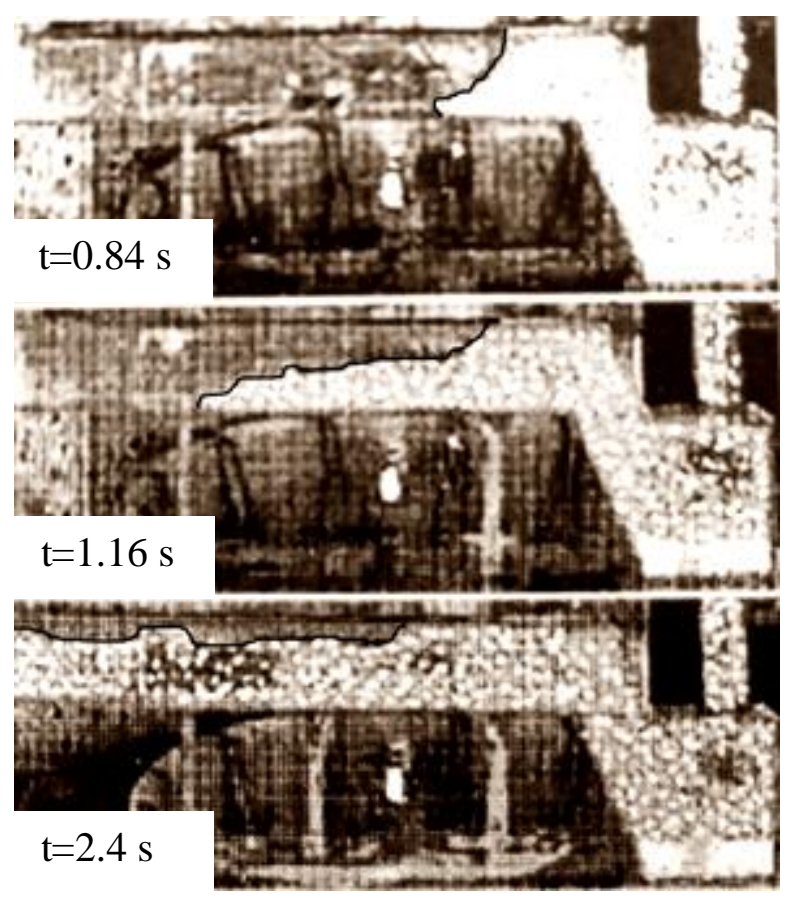

(a) Water solution flowing state
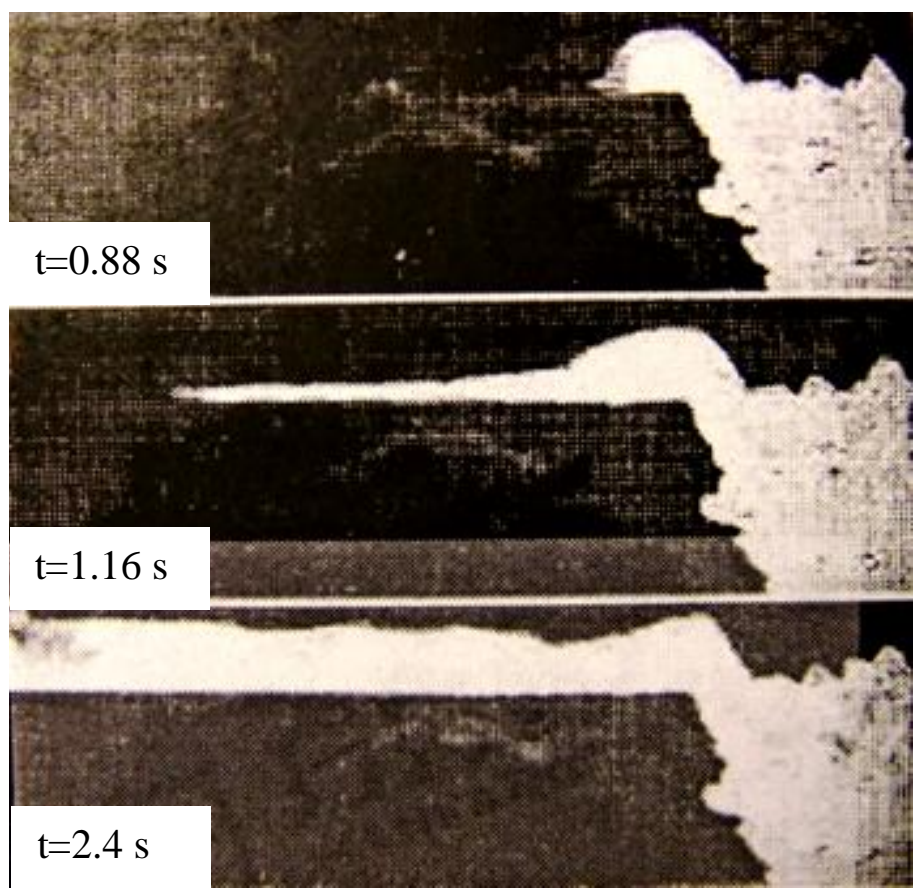

(b) Aluminum flowing state

Figure 2.13. Comparis on between the water solution flowing state and aluminum flowing state at different times [79] 
Other than allowing direct visualization of the flow pattern of liquid metals, water analogues also aid in the understanding the entrainment phenomena during casting [78,80], and therefore provide valuable information on the factors controlling the entrainment processes. For example, the use of water to simulate aluminum gravity casting has shown that the volume of air entrapment increases steadily with relatively small increases in the velocity of the liquid in the runner [78]. Hence an effective way to minimize the entrainment of air in this case is to decrease the velocity of the flow into the die cavity. By applying the same approach, the water analogue technique can assist in the design and optimization of casting systems, such as the runner and gating $[13,81,82]$.

Water models can be used as a simple means to validate mathematical models, owing to its advantages in flow observation and measurement. Both qualitative comparison, by flow patterns, and quantitative comparison, by filling rate assessments, can be carried out with relatively inexpensive equipment [12]. Once validated, the mathematical model can be applied to simulate aluminum filling with confidence.

In summary, water analogue experiments, when dynamic similitude is achieved, can predict the shape and movement of the liquid free surface in a casting process with acceptable accuracy. In some cases, exact dynamic similitude is not necessary. In particular, the study of the entrainment process or the validation of mathematical models can be achieved with approximate models, as is the case in the work described in this thesis. 


\section{SCOPE AND OBJECTIVE}

\subsection{Scope of Research Program}

The goal of this research program is to investigate the flow behavior during filling of the mould used to produce Low Pressure Die Cast (LPDC) aluminum automotive wheels. A study using a combination of both water analogue experiments and computational modeling was used to develop a fundamental understanding of the factors controlling the flow conditions that lead to the entrainment of oxide films and bubbles in a LPDC wheel. This knowledge will to aid in the optimization of industrial die geometry and process parameters to reduce the occurrence of flow related defects.

In the experimental portion of this study, a transparent die section based on the geometry of an industrial production die was manufactured to allow direct observation of fluid flow. A PID controlled pressure system was designed and assembled to fill the die cavity with water, based on the pressure system used in a typical LPDC machine. The air pressure in the die cavity and the predefined pressure curve are two primary factors that affect the filling and severity of turbulence. Thus, a set of die filling tests with different venting conditions and pressure curves was carried out to assess the flow's sensitivity to both factors. The filling process of the physical model was recorded with a video camera and the flow regime analyzed in terms of its potential to entrain the free surface and air bubbles. Further data such as the fill sequence and fill time were extracted from the filling video to gain further quantitative insight into the flow regime.

A mathematical model of the water analogue experiment was developed in the commercial CFD program, ANSYS CFX. Each experiment was studied with the model and a sensitivity analysis 
was performed to determine the critical model input parameters. These parameters were finetuned against data obtained via water modeling and the validity of the computational model was established by comparing the predicted filling states to the experiment.

\subsection{Objectives of Research Program}

The objectives of the present study are:

- To develop a water analogue experiment to replicate some aspects of the die filling process of a LPDC wheel. The experimental setup should facilitate the variation of relevant experimental conditions, including venting and filling pressure curve.

- To develop and verify a computational model capable of reproducing the flow behavior observed during die filling in the water analogue experiment. Once validated this computer model can be applied to the industrial casting scenario by implementing the process parameters and appropriate material properties applicable to the industrial process.

- To gain a fundamental understanding of the effects that venting and the predefined pressure curve have on the filling behavior and thus defect formation in LPDC wheels. 


\section{PHYSICAL MODEL}

The quality of die filling, which can be characterized by the free surface flow behavior, has a strong influence on the reliability of casting components $[8,83]$. The study of free surface flow during casting requires the observation and measurement of the flow occurring inside the die cavity. The main challenge lies in how to visualize the flow. As the steel die cavity used in wheel casting is opaque and geometrically complex, it is difficult to obtain flow data. Techniques such as real time X-ray imaging have been effective in imaging metal flow in cavities. Unfortunately, the X-ray-based methods are difficult and costly to undertake and therefore do not lend themselves to highly controlled systematic studies. Therefore, there is a need to simplify the the method and problem.

Water analogue experiments have been employed in many studies to investigate the liquid metal filling processes and their utility has been well established (as described in Section 2.4 Water Analogue Technique (Physical Model)). Due to the ability to conduct the experiments in transparent dies, water analogue experiments allow for direct flow observation and relatively easy quantification of date such as the evolution in fluid depth with time, the velocity of the fluid and the free surface profile. They overcome the difficulties associated with molten metal and tests can be conducted with relative ease, negligible cost and in a highly controlled manner.

\subsection{Experimental Setup}

The water analogue experimental setup contains two major elements, the transparent die cavity and the pressure control system. The transparent die was designed with the aid of preliminary analysis, following the rules that the die should allow for direct observation, easy measurements 
of the flow field and easy adjustment of the experimental conditions. The pressure control system was designed to achieve liquid feeding conditions that are similar to the industrial process. Modifications were made due to the differences in liquid properties and setup heights between the industrial and lab settings.

\subsubsection{Die Design}

There are several issues involved in the full 3D wheel die or any periodic sub-section of it. Firstly, part of the flow information may be missing or overlapping, regardless of how the camera is positioned. For example, when looking down the flow in a wheel cavity from the top, as shown in Figure 4.1 (a), most of flow phenomena occurring during rim filling will be missed. In Figure 4.1 (b) when looking at the rim towards the central axis of the wheel in a $3 \mathrm{D}$ transparent die, flow in the spokes and the rim on the opposing side of the wheel will also be visible. This makes differentiating between the desired flow and the background flow extremely challenging.

Secondly, the shape of the free surface and the severity of turbulence cannot be easily viewed or correctly characterized. This is especially true when examining a surface wave. When looking from above, the magnitude of the wave appears smaller, which leads to an underestimation of the severity of turbulence. Even if multiple cameras, positioned at different angles and locations are used, it is difficult to extract meaningful data from areas in a complex 3D die.

Thus, it was decided to use a 2D planar "pseudo" die section rather than a 3D replica of the production die cavity of the wheel. The planar die is referred to as 'pseudo' as its 2D profile approximates the wheel as axisymmetric with a geometrically simplified solid hub that extends 
out to the rim - e.g. it does not include the windows (refer to Figure 1.5 in Chapter 1) nor does it exhibit periodic symmetry.

The $2 \mathrm{D}$ planar pseudo die section was made by extracting a $2 \mathrm{D}$ profile from the full $3 \mathrm{D}$ geometry of a production die, as shown in Figure 4.2. The 2D profile includes not only the profile of the wheel but also the profiles of the sprue and riser.

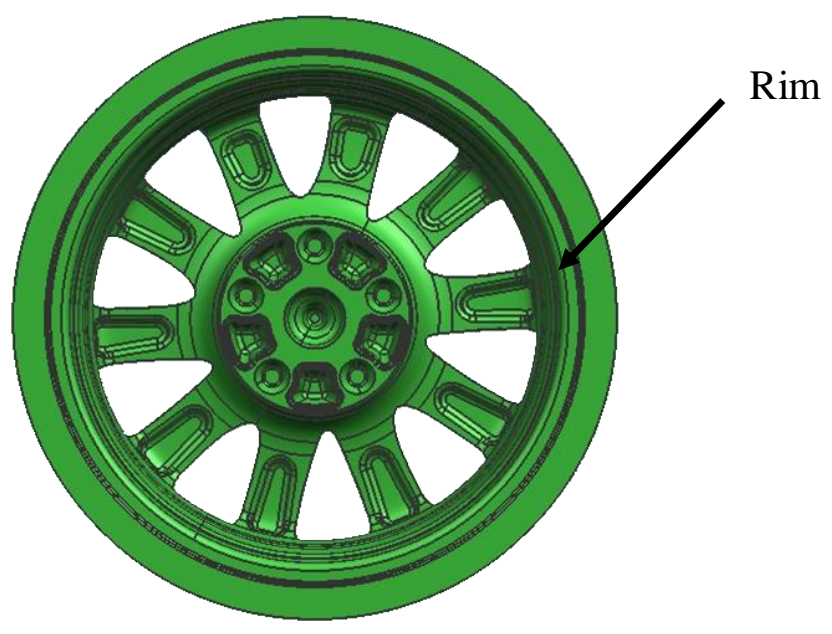

(a) Top vie w of a ten-spoke wheel

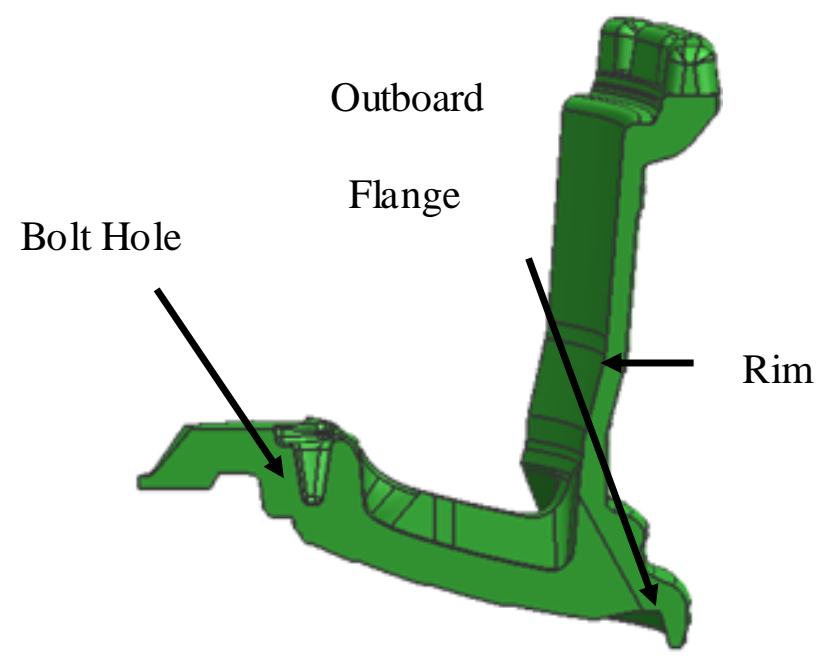

(b) Side vie w of a periodic section $\left(36^{\circ}\right.$ we dge $)$ from a five-spoke wheel

Figure 4.1. CAD models of wheels 


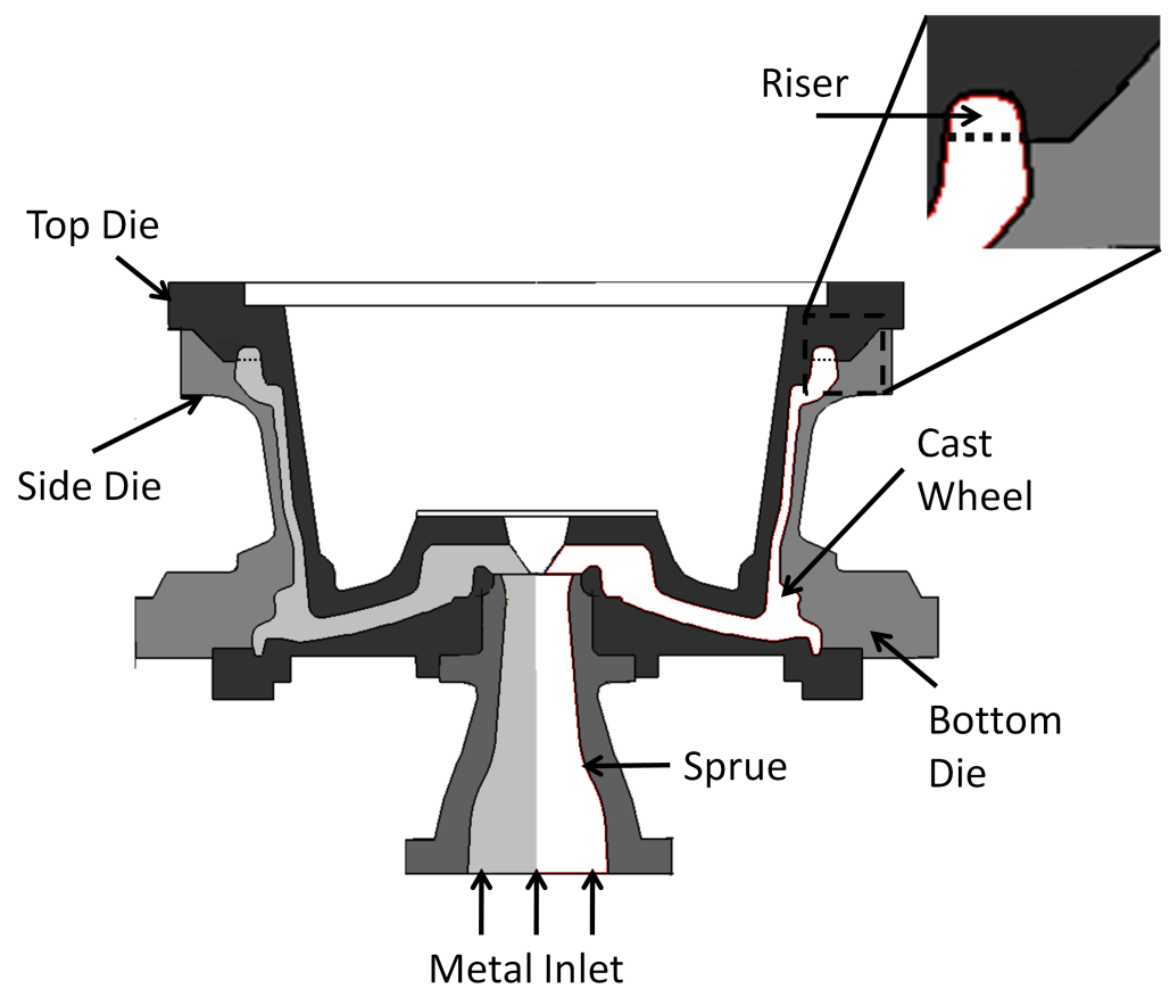

Figure 4.2. Side vie w of a die -tooling assembly for typical LPDC process, with a 2D profile of both the die and the sprue highlighted (the right brighter part)

\subsubsection{Pseudo Die Thickness Determination}

The pseudo die was constructed by machining the axisymmetric wheel profile, show highlighted in white in Figure 4.2, from a plexiglass sheet. This was done using a water jet by Tana Industries Ltd. in Delta, B.C., Canada. The sheet was then sandwiched between two additional sheets of plexiglass to form a watertight cavity for conducting the tests. In the production die, friction acting on a plane normal to the circumferential direction does not influence flow up the rim, whereas in the 2-D water model there will be some resistance resulting from the flow contacting the plexiglass sheets on either side of the cavity. The influence of wall resistance on the flow within the rim was determined with the aid of a computational study on a simplified geometry (Figure 4.3). A CFD model of a $10 \mathrm{~mm}$ thick die section with free-slip wall conditions 
on the side walls was run and used as the baseline case. The free-slip wall condition results in no wall friction but restricts flow normal to the surfaces and thus would behave similar to the production rim. Two other cases, which included the wall shear stress (no slip), were run for both a $10 \mathrm{~mm}$ and $20 \mathrm{~mm}$ thick cavity for comparison.

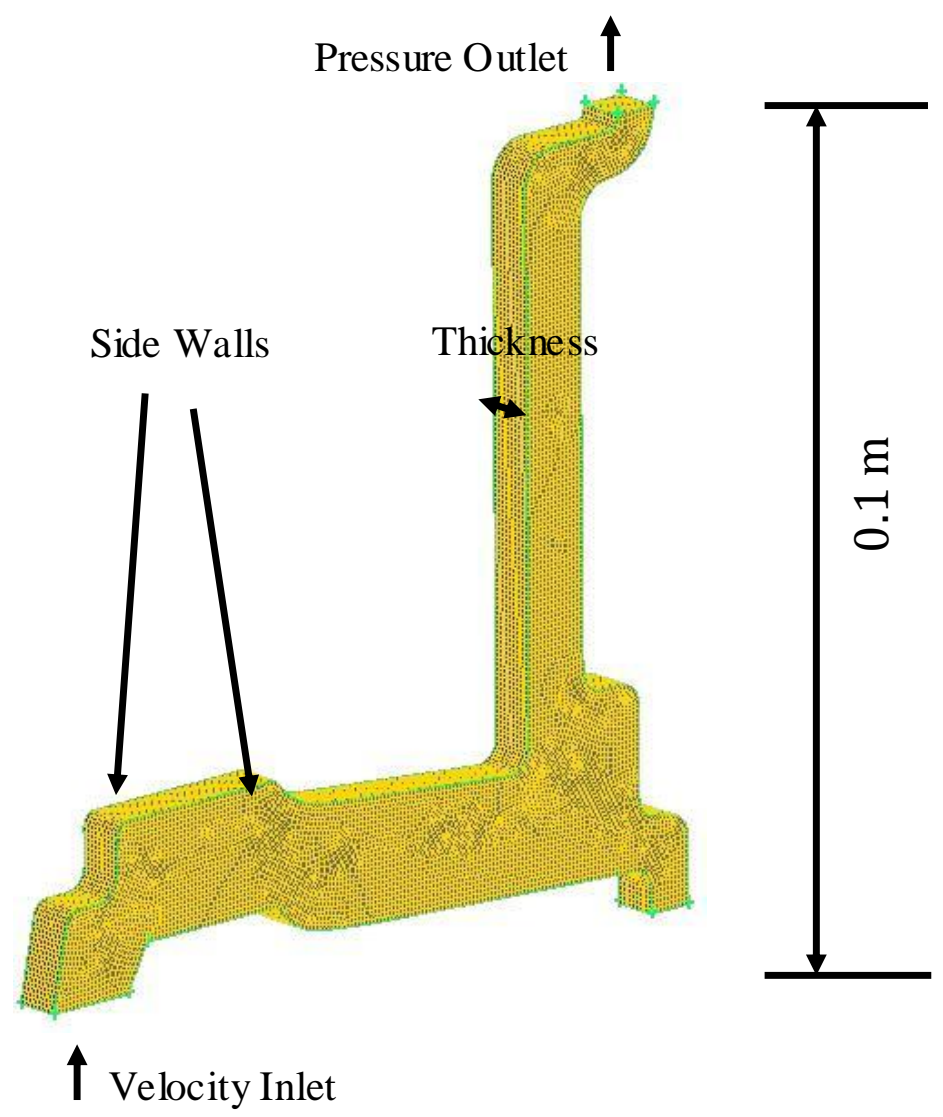

Figure 4.3. An extrusion of a simplified wheel profile, with $1 \mathrm{~mm}$ hexahe dron grids

A velocity inlet was applied to the model and was selected as the minimal velocity estimated from the production pressure curve. Based on the geometry of channel at the inlet, this velocity gives a Reynolds number of 230 for the $10 \mathrm{~mm}$ extrusion and 350 for $20 \mathrm{~mm}$ extrusion at the inlet, which ensures a laminar flow condition at the inlet and highlights the wall effect. The outlet section was defined as a pressure outlet with an average pressure of $1 \mathrm{~atm}$. The model 
considered single phase (water), fully saturated, isothermal flow. A time step size of $1 \mathrm{~s}$ was used with 30 iterations per time step.

Flow profiles at five cross sections for cases with free-slip wall boundaries and no-slip wall boundaries are shown in Figure 4.4. For the case with free-slip wall boundaries (Figure 4.4 (a)), there is no velocity gradient in $\mathrm{z}$ direction, indicating that the two sidewalls have no effect on the flow. In the cases with no-slip wall boundaries (Figure 4.4 (b) and (c)), the velocities are greatest at the center and zero at the no-slip walls. Clearly the presence of the sidewalls will affect the flow field to some degree and this will need to be considered when interpreting the results. In $20 \mathrm{~mm}$ thickness case, the flow profile in the centre of the cavity away from the sidewalls approaches the free-slip case. 


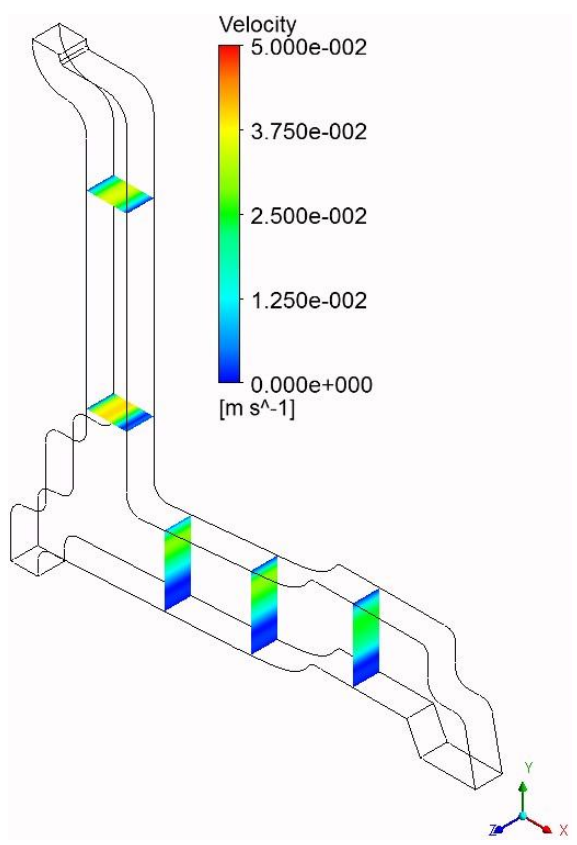

(a) Free-slip wall condition (10 $\mathrm{mm}$ thick)

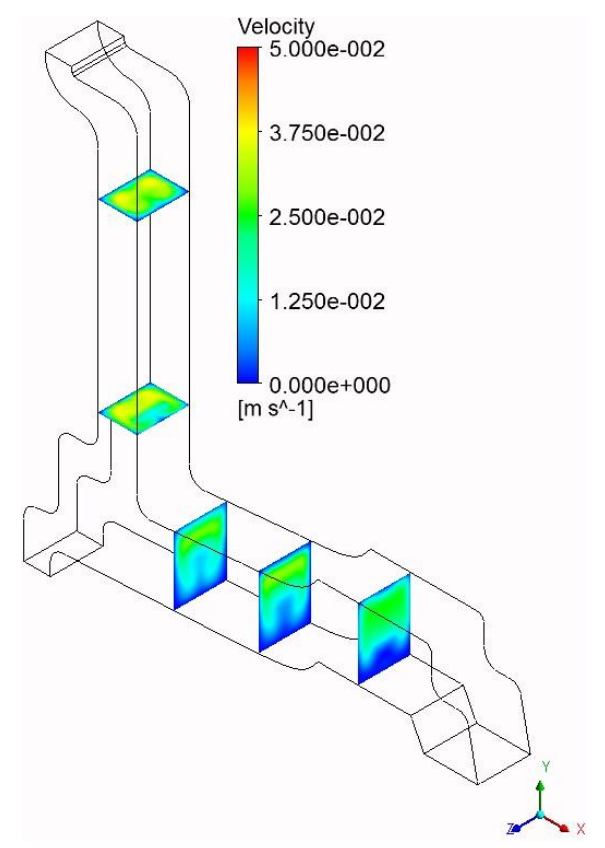

(c) No-slip wall condition ( $20 \mathrm{~mm}$ thick)

Figure 4.4. Flow pre dictions at five locations in the model domain for free-slip wall boundary conditions and no-slip wall boundary conditions with diffe rent thicknesses 


\subsubsection{Water Test Die}

Due to the standard sizing of plexiglass plate available commercially the cavity thickness ended up being $18 \mathrm{~mm}$ in the water model. Figure 4.5) shows a photograph of the resulting pseudo die assembly. The assembly was bolted together and sealed by an O-ring along the profile. Holes ( $1 \mathrm{~mm}$ in diameter) were drilled at locations in the hub, the outboard flange, and the inboard flange respectively, as these locations correspond to venting points in the production die where the ejection pins and the die parting lines are located (Figure 4.5). In the industrial process the riser area above the inboard rim flange is removed after casting, therefore it is not necessary for the region to fill completely. However, the material contained in the riser is used to feed the rim section to prevent shrinkage porosity. Therefore inadequate filling of the riser due to lack of air venting in this area would result in insufficient feeding of liquid metal to offset the volumetric shrinkage associated with solidification in the neighboring areas. To enable venting in this area to be evaluated, an extra vent was added on the top of the riser in the water model. Drain cocks were installed on the vents to allow different venting resistances to be studied. The vents and drain cocks were added by the technical staff in the Department of Materials Engineering, the University of British Columbia. Paper with $5 \mathrm{~mm}$ grid squares was fixed to the back of the water test die to ease tracking of the liquid front and to aid in quantification of local flow velocities and the locations and extent of regions of air entrainment. 


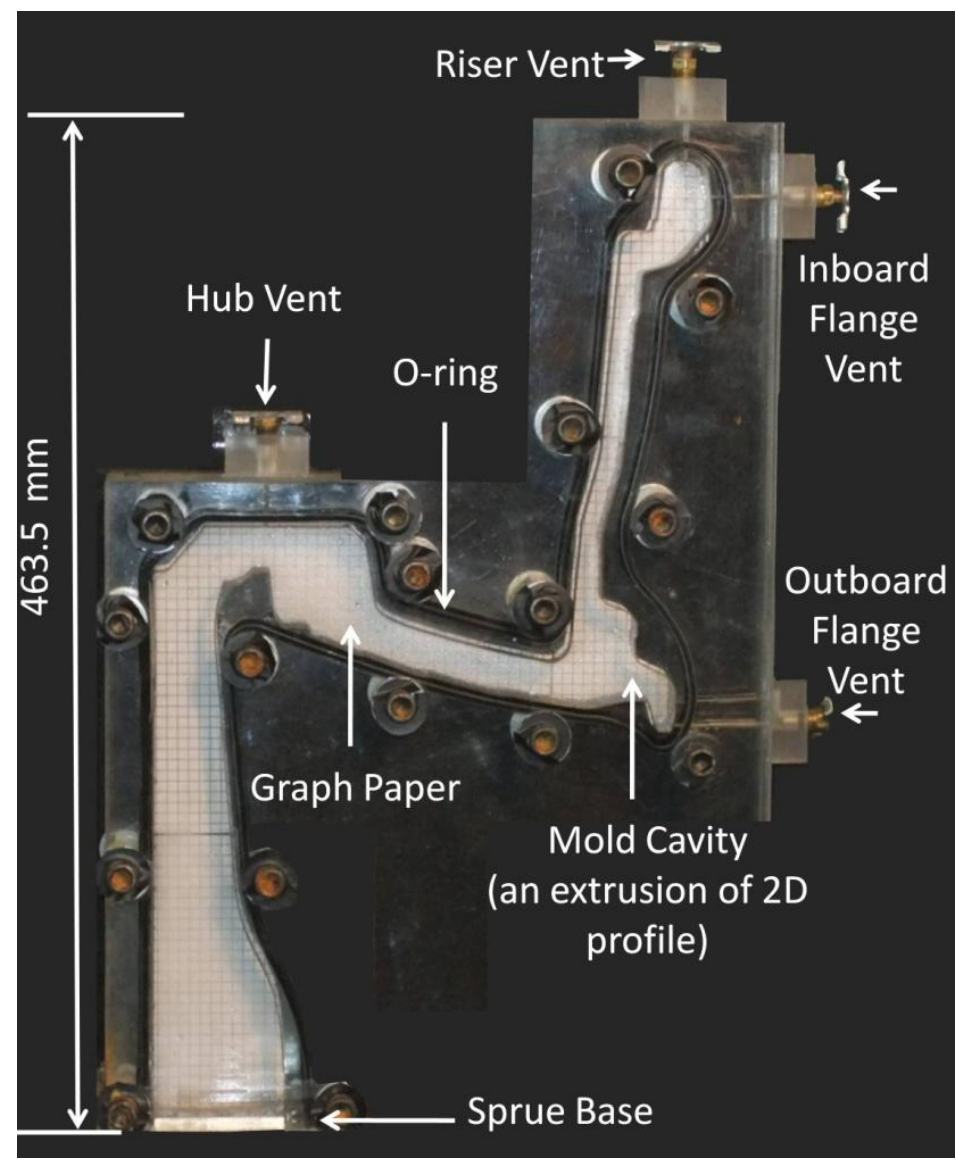

Figure 4.5. A transparent water model made of plexiglas

\subsubsection{Transition Pipe Design}

One consequence of having a planar die section is that the sprue base is also rectangular in crosssection. A transition pipe was required to adapt the rectangular sprue base to the round inlet pipe coming from the water pressure control system. This is similar to the joint pipe in a typical die casting machine which links the holding furnace with the sprue. An analysis was completed to determine the transition pipe length so as to minimize any turbulence associated with the transition. 
After two iterations the design shown in Figure 4.6 was arrived at. It has a rectangular lip at the top that fits into the rectangular sprue base and a round threaded hole at the bottom which fits a $12.7 \mathrm{~mm}(1 / 2$ ") pipe fitting.
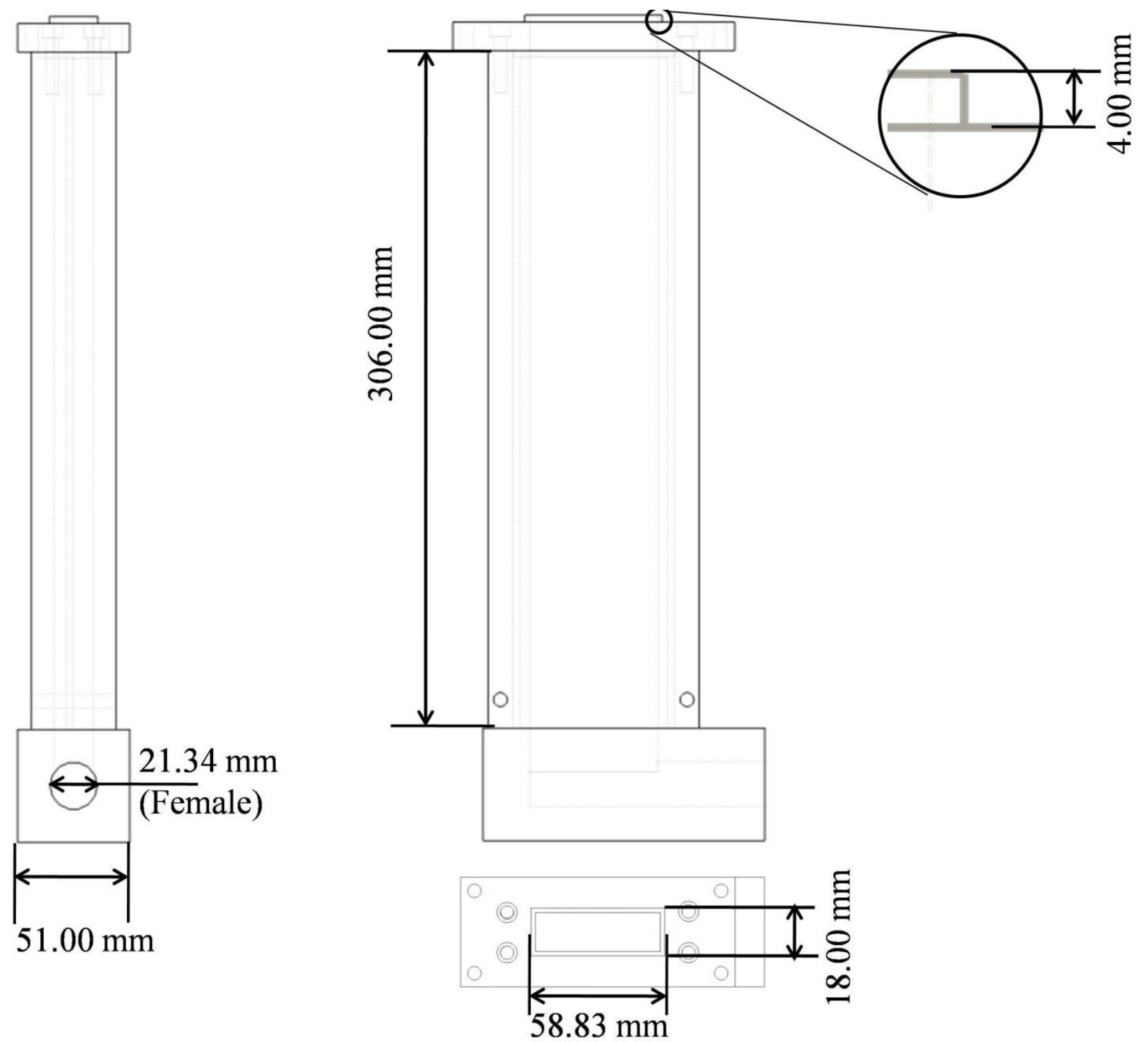

Figure 4.6. Drawings of the transition pipe (courtesy Ross McLeod)

\subsubsection{Pressure Control System}

In the LPDC process, the air pressure in the holding furnace, as seen in Figure 1.3 of Chapter 1, that pushes liquid metal up follows a pressure curve. This technique requires a closed-loop 
control circuit and a pressure sensor to adjust the pressure in the holding furnace to the set-point values. The circuit traditionally contains three elements: a control valve that regulates the air flow and thus the air pressure in the holding furnace, pressure sensors for assessing the pressure, and a programmable controller. The controller adjusts the position of the valve based on the deviation between the real time pressure and the set-point at each point in the pressure curve. Instead of pressurizing a reservoir of water with air, the experimental setup for this study was directly connected to a water supply and the water pressure at the bottom of the transition pipe (similar to the joint pipe in Section 4.1.2) was measured and controlled. A schematic of the pressure control system developed for this study is illustrated in Figure 4.7. The pressure sensor, which detects the real time pressure information at the bottom of the transition pipe, is read by the data acquisition board. This information is then assessed by a Proportional - IntegralDerivative (PID) control program based in LabVIEW. The PID control program compares the pressure to the set-point, calculates the error, and then decides on a corrective action, which is an input to the control valve. 


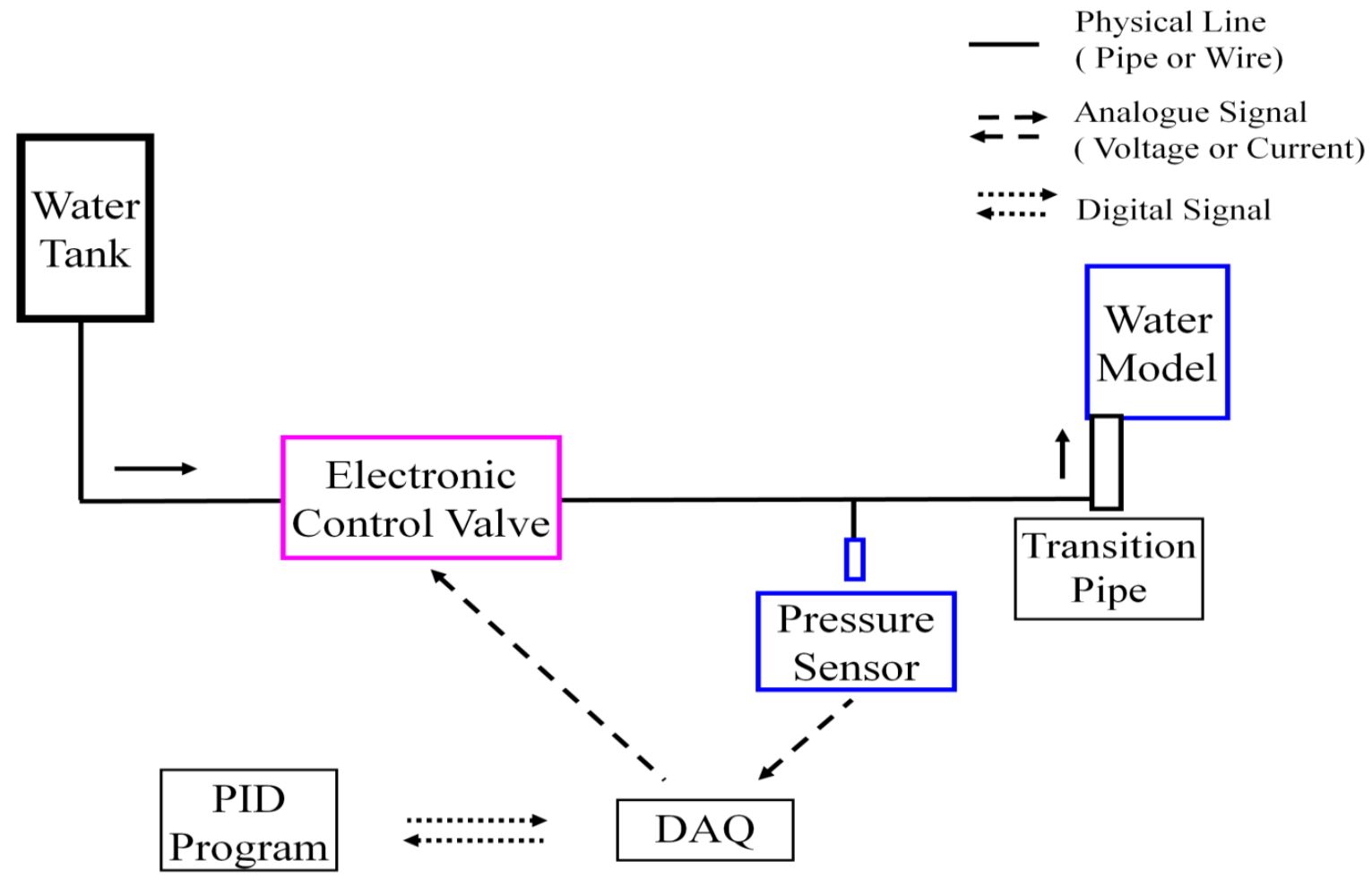

Figure 4.7. Schematic of the implemented pressure control system

\subsubsection{Electronic Control Valve}

The size of the electronic control valve was selected based on the flow coefficient $\mathrm{C}_{\mathrm{v}}$. The $\mathrm{C}_{\mathrm{v}}$ calculates the relative flow-efficiency of the valve. It can be described by the following equation [84]

$$
C_{v}=Q \sqrt{\frac{S G}{\Delta P}}
$$

where $Q$ is the flow rate (US gallons per minute), $S G$ is the specific gravity, and $\Delta P$ is the pressure drop across the valve (psi). 
The pressure of the water supply to the laboratory is nominally $60 \mathrm{psi}$, and based on the required flow rate estimated from the data provided by one of the major wheel manufacture in North America, the maximum necessary $\mathrm{C}_{\mathrm{v}}$ is around 0.26 . Thus in order to achieve sufficient flow rate, the $C_{v}$ of the valve chosen in this study should be larger than 0.26 .

Another important parameter used in choosing a valve is the maximum differential pressure across the valve. For a valve that is designed to close against the supply pressure, the valve mechanism will stall if the supply pressure gets too high, due to the fact that the motor will not have enough torque to close the valve. Therefore, for the valve to operate normally, its maximum differential pressure needs to be larger than the maximum supply pressure in the lab which is 60 psi.

In this study, the electronic control valve, ECV-250B - 4X made by Hass Manufacturing Company, was used. It has a flow coefficient of 0.6 and a maximum differential pressure of 120 psi. It is a computer-controlled proportional valve which directly accepts a $1-5 \mathrm{~V}$ DC control signal. The actuator is a DC step motor that has 200 steps / revolution. It takes 4 revolutions and approximately 5 seconds to actuate from the fully closed position to the fully open position, which amounts to $6.25 \mathrm{~ms}$ per step [85].

\subsubsection{Pressure Transducer}

The range of gauge pressure experienced in the water analogue experiment is $0 \mathrm{kPa}$ to $8.22 \mathrm{kPa}$ (detailed calculations can be found in Section 4.1.4). Based on this information, a silicon-based pressure sensor with a pressure range from $0 \mathrm{kPa}$ to $10 \mathrm{kPa}$ was selected. This sensor is an MPVZ Series from Freescale Semiconductor, which is a piezoresistive transducer that requires an external voltage to operate. A summary of the operating characteristics are listed in Table 4.1. 
Table 4.1. Ope rating Characte ris tics [86]

\begin{tabular}{|c|c|c|c|c|}
\hline Characteristic & Min & Typical & Max & Unit \\
\hline Pressure Range & 0 & - & 10 & $\mathrm{kPa}$ \\
\hline Supply Voltage & 4.75 & 5.0 & 5.25 & Vdc \\
\hline $\begin{array}{c}\text { Minimum Pressure Offset }{ }^{(1)} \\
@ V_{s}=5.0 \text { Volts }\end{array}$ & 0 & 0.2 & 0.425 & Vdc \\
\hline $\begin{array}{l}\text { Full Scale Output } \\
\text { @ } \mathrm{V}_{\mathrm{s}}=5.0 \text { Volts }\end{array}$ & 4.475 & 4.7 & 4.925 & Vdc \\
\hline $\begin{array}{l}\text { Full Scale } \operatorname{Span}^{(3)} \\
\text { @ } \mathrm{V}_{\mathrm{s}}=5.0 \text { Volts }\end{array}$ & 4.275 & 4.5 & 4.725 & Vdc \\
\hline Accuracy & - & - & \pm 5.0 & $\% \mathrm{~V}_{\mathrm{FSS}}$ \\
\hline Sensitivity $^{(4)}$ & - & 450 & - & $\mathrm{mV} / \mathrm{kPa}$ \\
\hline Response Time ${ }^{(5)}$ & - & 1.0 & - & $\mathrm{ms}$ \\
\hline Warm-Up Time ${ }^{(6)}$ & - & 20 & - & $\mathrm{ms}$ \\
\hline
\end{tabular}

1. Offset is defined as the output voltage at the minimum rated pressure.

2. Full Scale Output is defined as the output voltage at the maximum or full rated pressure.

3. Full Scale Span $\left(\mathrm{V}_{\mathrm{FSS}}\right)$ is defined as the algebraic difference between the output voltage at full rated pressure and the output voltage at the minimum rated pressure.

4. Sensitivity is defined as the ratio of voltage to pressure (V/P).

5. Response Time is defined as the time for the incremental change in the output to go from $10 \%$ to $90 \%$ of its final value when subjected to a specified step change in pressure.

8. Warm-up Time is defined as the time required for the product to meet the specified output voltage after the pressure has been stabilized.

\subsubsection{Data Acquisition (DAQ) Board}

As shown in Figure 4.7 and based on the specifications of both the electronic control valve and the pressure transducer, the DAQ board was required to receive and send voltage signals equal or larger than 5 V. A USB - 6009 from National Instruments was used in this study and its 
specification is shown in Table 4.2. The DAQ has an external voltage output of $5 \mathrm{~V}$ which directly powers the pressure transducer.

Table 4.2. Spe cifications of USB - 6009 DAQ board

\begin{tabular}{ccc}
\hline Signal Name & \multicolumn{2}{c}{ Specifications } \\
\hline Analogue Input (AI) & Input Range & $\pm 10 \mathrm{~V}$ single-ended \\
& Number of AIs & 8 single-ended \\
& Input Resolution & 13 bits single-ended \\
& Max Sampling Rate & $48 \mathrm{kS} / \mathrm{s}$ \\
Analogue Output (AO) & Output Range & 0 to $+5 \mathrm{~V}$ \\
& Number of AOs & 2 \\
& Output Resolution & $12 \mathrm{bits}$ \\
& Max Update Rate & $150 \mathrm{~Hz}$ \\
External Voltage & & $+5 \mathrm{~V}$ typical (up to $200 \mathrm{~mA})$ \\
\hline
\end{tabular}

\subsubsection{Control Program Development}

There are three basic types of controllers, proportional $(\mathrm{P})$, proportional-integral (PI), and proportional - integral - derivative (PID), with PID being the most complex [87]. For this project, a PID algorithm was used. When using the PID control, a process variable and a set-point must be specified. The process variable is the system parameter to be controlled, such as pressure, temperature, or flow rate. The set-point is the desired value for this parameter. A PID controller determines a controller output value, such as valve position or heater power. When applied in a process the controller output value drives the process variable toward the set-point [88].

The equation describing the output from a PID controller is [88] 


$$
\mathrm{u}(\mathrm{t})=\mathrm{K}_{\mathrm{c}}\left(e+\frac{1}{\mathrm{~T}_{\mathrm{i}}} \int_{0}^{\mathrm{t}} \mathrm{edt}+\mathrm{T}_{\mathrm{d}} \frac{\mathrm{de}}{\mathrm{dt}}\right)
$$

where $e$ is the error, which is the deviation between the set-point and the process variable, $K_{c}$ is the controller gain, $T_{i}$ is the integral time in minutes, and $T_{d}$ is the derivative time in minutes. The three terms must be tuned to obtain satisfactory control.

The proportional component, adjusted by the controller gain $\mathrm{K}_{\mathrm{c}}$, changes the output proportional to the current error. Large values typically mean a faster response.

The integral term is proportional to both the magnitude of the error and the duration over which the error is assessed. It accelerates the movement of the process towards the set-point and eliminates the residual steady-state error more quickly. Since the integral term responds to the accumulated error from the past, the integral action may lead to an overshoot of the setpoint.

The derivative part of the PID algorithm anticipates future behavior of the error by calculating the derivative of the error. A large value decreases the tendency to overshoot. The trade-off is that it slows the transient response of the controller and may lead to instability due to signal no ise amplification in the differentiation of the error [87].

A control program shown in Figure 4.8 was developed within LabVIEW. The set-point profile is entered in Block A. The first column is time while the second column is the corresponding voltage at that time which is calculated from the predefined pressure curve. Data between these points is interpolated linearly. Block B is the PID controller. The three numbers below the PID icon, 40, 0.03, and 0.01 represent the controller gain, the integral time, and derivative time respectively. The PID parameters were tuned using a trial and error process, with basic 
knowledge of the effects of each parameter on system response, obtained from the literature [87]. The DAQ Assistant in Block $\mathrm{C}$ collects data from the pressure transducer. The sampling rate is set to $1 \mathrm{kHz}$ but the output is averaged over every 20 samples to minimize the noise. The averaging operation leads to an overall sampling rate of $50 \mathrm{~Hz}$, which confirms to the sampling rate used in the production process. Block $\mathrm{D}$ is the signal output interface that sends the controller output to the electronic control valve. The other parts of this program display and record relevant data.

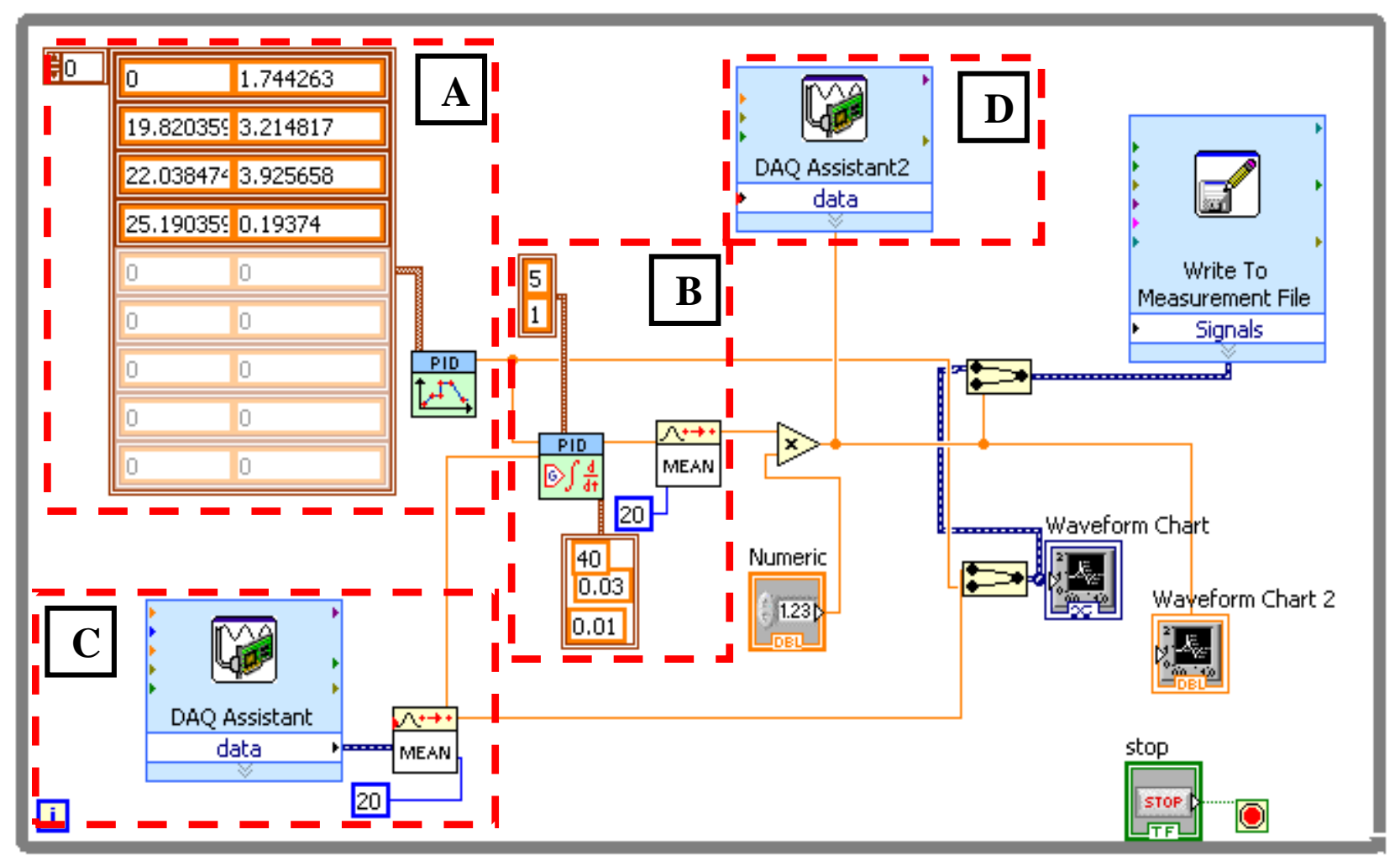

Figure 4.8. The PID control program developed within LabVIEW. A is the setpoint profile, $B$ is the PID controller, $C$ is the DAQ assistant and $D$ is the signal output interface. 


\subsubsection{Image Recording}

The die filling processes in the water analogue experiment were recorded with digital video cameras. Two cameras, a digital point-and-shoot camera (FinePix F75EXR from FUJIFILM) and a Gigabit Ethernet machine vision camera (GC 1290 from Allied Vision Techonologies Canada Inc.), were used. The FinePix F75EXR was used to record a macro view with an image size of $640 \times 480$ pixels at 30 fps. Information such as fill sequence, fill time and free surface shape were analyzed through the macro view. The GC 1290 was used to record a close-up view of specific areas of interest. Frame sizes of $1280 \times 480$ pixels, $960 \times 480$ pixels, and $640 \times 480$ pixels were used for different regions, depending on the dimensions of that region. The frame rate for the close-up view was 59 fps.

\subsection{Test Conditions}

Experiments were performed to investigate the effects of different parameters on the flow behavior during die filling. Five tests with different venting conditions (Table 4.3) were carried out. Since the relationship between the number of turns and the amount of opening of the drain cocks installed on the vents are unknown, the drain cocks were left either fully closed or fully open to provide repeatable vent conditions.

The pressure curves used in the water analogue experiments were developed from a production pressure curve supplied by a North America wheel manufacture. The pressure curves were modified so that liquid levels in the water analogue experiment evolved approximately the same as the liquid aluminum levels during in production die. The differences in density between water and liquid A356 aluminum alloy and in the setup heights between the die casting machine and 
the water analogue experiment were taken into account to adjust the pressure. Figure 4.9 shows an example of the A356 pressure curve and the resulting water pressure curve. The slope difference between the curves is caused by the density difference between the two fluids. As this study is only concerned with the filling section of the pressure curve the water curve ends at 30 s whereas in the commercial A356 curve an over-pressure is applied to help avoid shrinkage.

In the die filling section, the metal height is related to the basic hydrodynamic equation $\mathrm{P}=\rho \mathrm{gh}$, where $P$ is pressure $(\mathrm{Pa}), \rho$ is liquid density, $g$ is gravitational acceleration $\left(\mathrm{m} / \mathrm{s}^{2}\right)$, and $h$ is the liquid height. Initial testing with the water analogue setup has shown that there is a delay in the actual liquid height compared to that expected by the values of the pressure curve. This is probably due to the back pressure built up in the die cavity during die filling that has reduced the fill rate. It was also observed that extending the fill time by $\sim 4 \%$ ensures that the die is fully filled at the end of the pressure cycle. Therefore, the pressure curves used in the water analogue experiment were all extended $4 \%$ in time. 


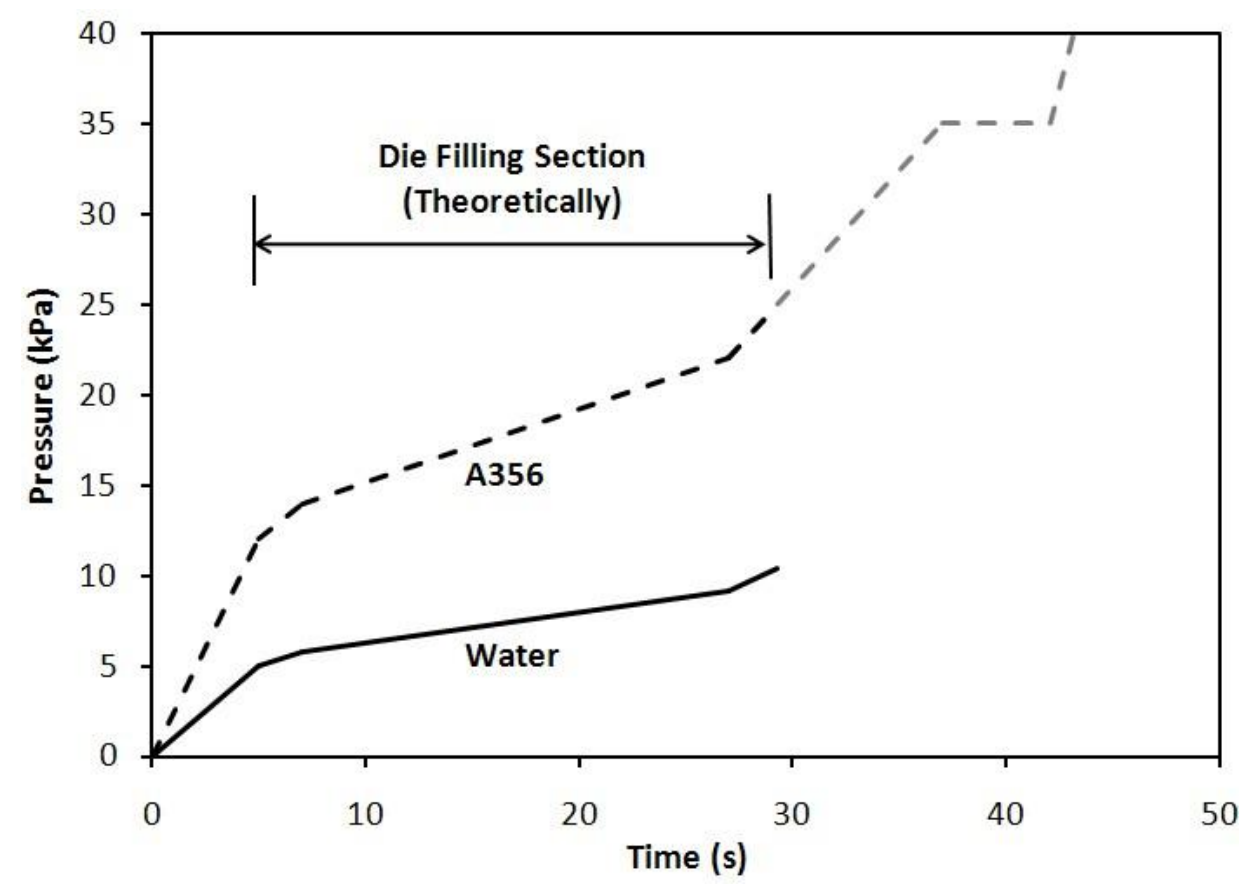

Figure 4.9. An example of the pressure curves for A356 and water

All the filling experiments started statically with an initial liquid height of $20 \mathrm{~mm}$ measured from the sprue bottom, in order to maintain the same initial condition between the different tests and for the computational model, which will be discussed in detail in the next chapter. Figure 4.10 shows an example of the predefined experimental pressure curve and the measured real time pressure curve. In general, the real time pressure curve controlled by the PID program follows the predefined one. 


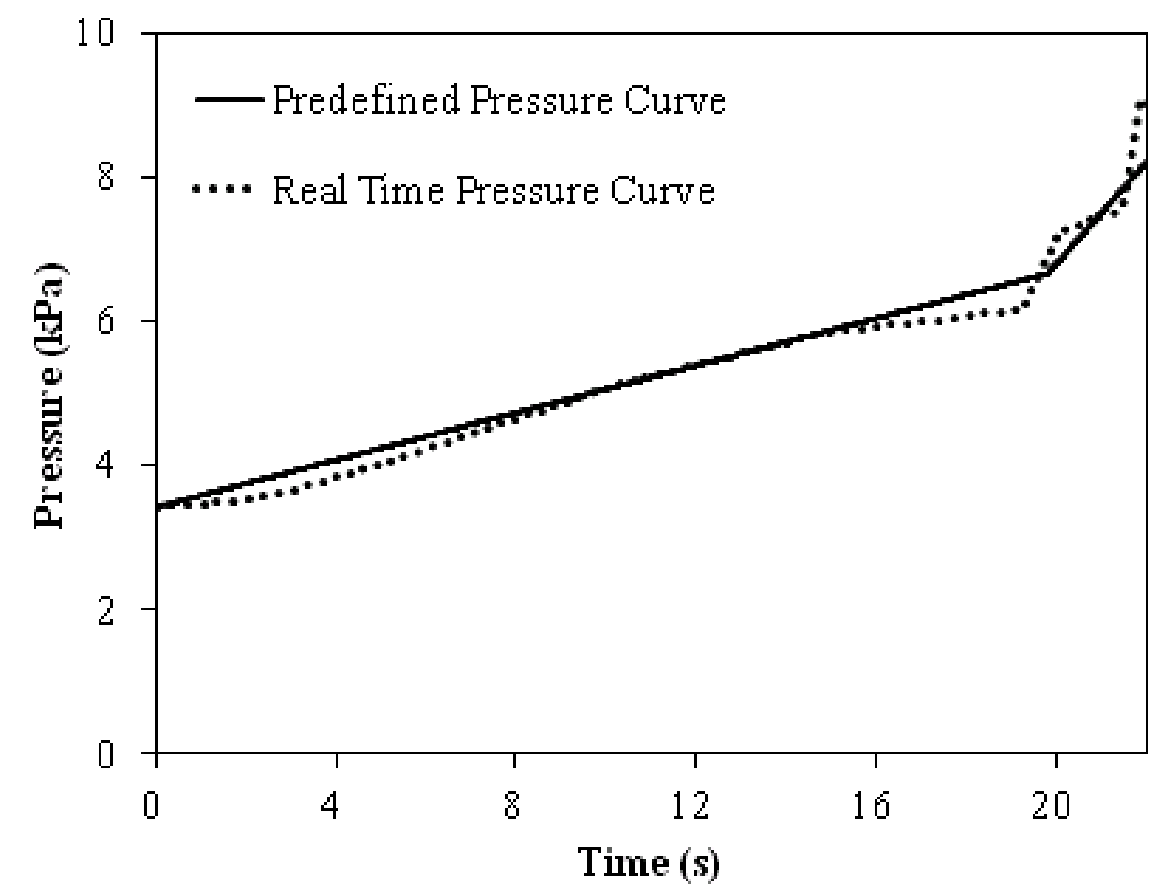

Figure 4.10. An example of the predefined and real time pressure curves

\subsubsection{Study of Venting Conditions}

The four vents in the water test die, which correspond to the locations of different gaps in the production die, are shown in Figure 4.5. The test matrix for the study of venting conditions can be seen in Table 4.3. The experimental matrix was carefully designed to evaluate the relative importance of each vent, except for the outboard flange vent whose contribution to venting is negligible because this area of the die fills very quickly.

During the die filling process in industry, the liquid aluminum in the die cavity does not pass into the venting gaps. This is due to a number of factors including the small clearances, the high surface tension of liquid aluminum, and that contact with the colder die will cause any aluminum that enters the gap to solidify. The containment of fluid in the water analogue experiment does not occur when the vents are open. Designing and implementing an automated vent closing system adds unnecessary complexity to the study. Instead vents were left open and the water 
allowed to flow out of the cavity. To assess the important of this, the water flowing through the hub vent was collected. When the inboard and outboard flange vents are closed, the hub vent is the only exit for water before the die is fully filled. The result showed that the fluid loss during die filling was only $1.18 \%$ of the total volume of fluid that fills the cavity. This is a negligible amount and therefore, leaving the vents fully open is a reasonable simplification of the venting conditions in an actual casting process.

Table 4.3. Venting conditions for different tests

\begin{tabular}{|c|c|c|c|c|}
\hline Test \# & Hub Vent & $\begin{array}{c}\text { Outboard } \\
\text { Flange Vent }\end{array}$ & $\begin{array}{c}\text { Inboard } \\
\text { Flange Vent }\end{array}$ & Riser Vent \\
\hline$\# 1$ & & & & \\
\hline \#2 & & & & \\
\hline \#3 & & & & \\
\hline$\# 4$ & & & & \\
\hline \#5 & & & & \\
\hline
\end{tabular}

\subsection{Experiment Procedure}

Experience while commissioning the pressure transducer has shown that pressure transducer calibrations done at different times during the day result in different transfer functions between the sensor voltage outputs and the pressure values, due to the error of the pressure transducer, the variations in water temperature and the ambient pressure, etc. Thus, the pressure transducer was recalibrated before every set of experiments. Eight different static liquid heights were used for calibration. The corresponding voltage readings were recorded. In order to reduce the measurement noise, more than 4000 samples were collected for each liquid height at the 
sampling rate of $1000 \mathrm{~S} / \mathrm{s}$, and the averaged voltage values were used to generate the transfer function. An example of the calibrated transfer function is presented in Figure 4.11, where $V_{\text {out }}$ is the voltage output of the pressure transducer and $P$ is the corresponding pressure.

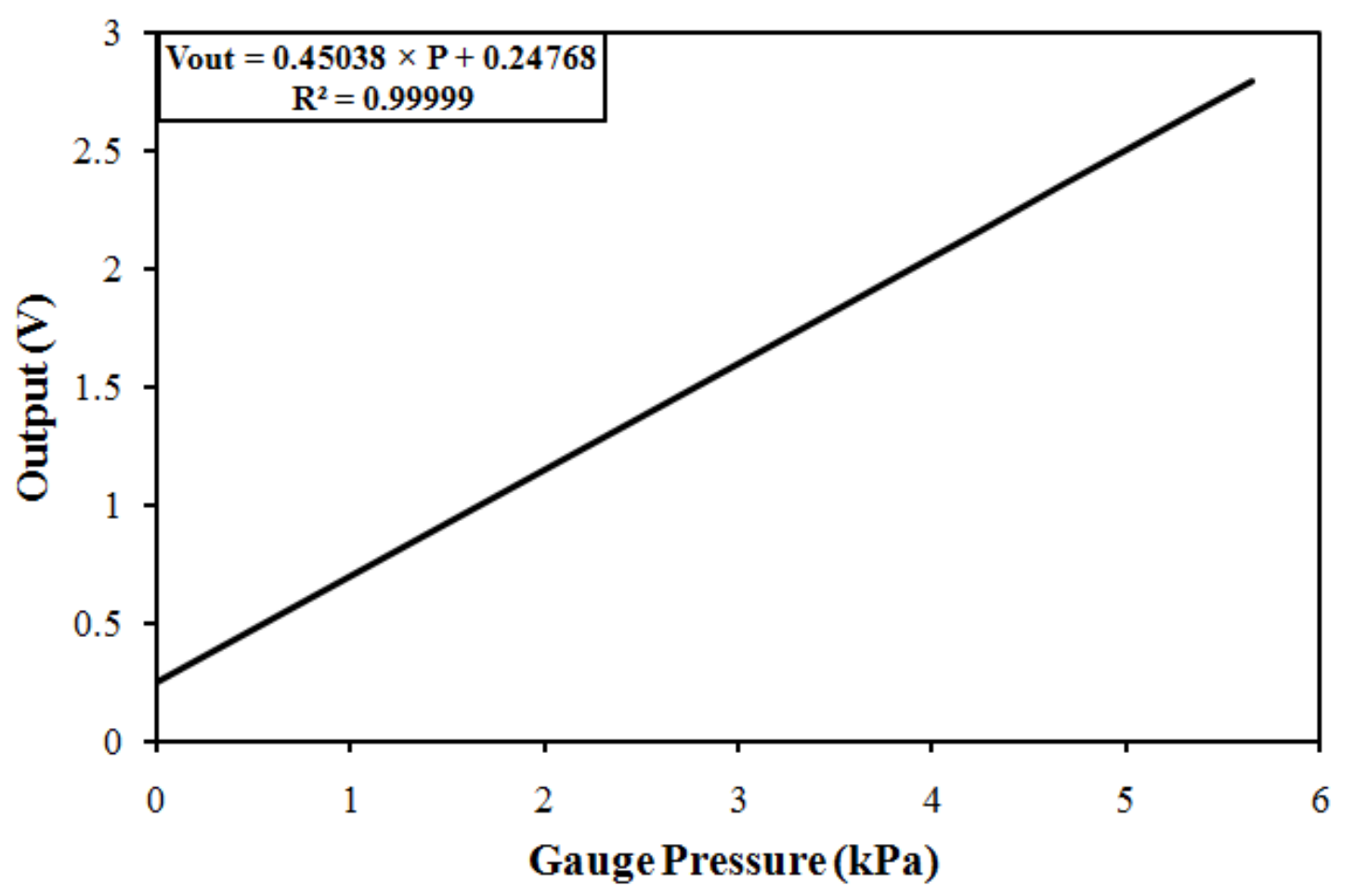

Figure 4.11. The calibrated transfer function

A stable pressure supply is essential to the repeatability of the filling experiment, as indicated by the many experimental results from the trial-and-error process in this study. Thus, the liquid height in the water tank, as shown in Figure 4.7, was kept at the same level at the start of every experiment. Each test condition was performed four times to evaluate the repeatability of the water analogue experiment. Meanwhile, one macro view and one close-up view were taken during each run. Therefore, for each test condition, there are four macro view videos and four close-up view videos. The close-up views were taken at four different locations, one per each run. These locations are: 1) the middle section of the water test die which includes the hub, the spoke, 
the outboard flange and the bottom of the rim, 2) the hub area, 3) the spoke area and 4) the outboard flange area.

After each test, the water in the die cavity was drained to the initial liquid height. The vents that remained open during the filling were cleared by pushing air through them. And the position of the camera GC 1290 was adjusted to record the close-up view for next die region.

\subsection{Experiment Results}

\subsubsection{Experiment Accuracy}

The experimental repeatability was assessed by putting the four, macro-view, filling videos of the same test condition side-by-side to compare the variation in water height with time. With the experiment procedure described in Section 4.3, the repeatability shown in Figure 4.12 was achieved. It was assessed for the Test \#1 condition with the original pressure curve. The red lines indicate where the free surfaces or the liquid levels are. The liquid levels in the four tests are close to each other, and the shapes of the free surfaces are also similar. Experience with the experimental set-up has shown that this high degree of repeatability could only be achieved when the four runs were conducted within a short time period. If the runs were conducted on different days or if tests with different experimental conditions were carried out in between, the repeatability was undermined. Liquid height differences as large as half of the height of the hub were often seen. A number of factors could have contributed to this behaviour. For example, as shown in Section 4.1.3.2, the accuracy of the pressure transducer is $\pm 5 \% \mathrm{~V}_{\mathrm{FSS}}$, which amounts to $\pm 500 \mathrm{~Pa}$. Although repeated measurements to a fixed static liquid height exhibited a maximum offset of only $24.4 \mathrm{~Pa}$, pressure discrepancy could be larger during the transient 
process of die filling. Variations in water density which occur when testing at different times may also play a role. Water temperature on different days or at different times during the day, i.e. mornings or evenings, is different and affects the water density and thus the water pressure head. Pressure transducer calibrations undertaken at two different times showed a maximum deviation of 55.64 Pa for the same output voltage. 
Run \#1

Run \#2

Run \#3

Run \#4
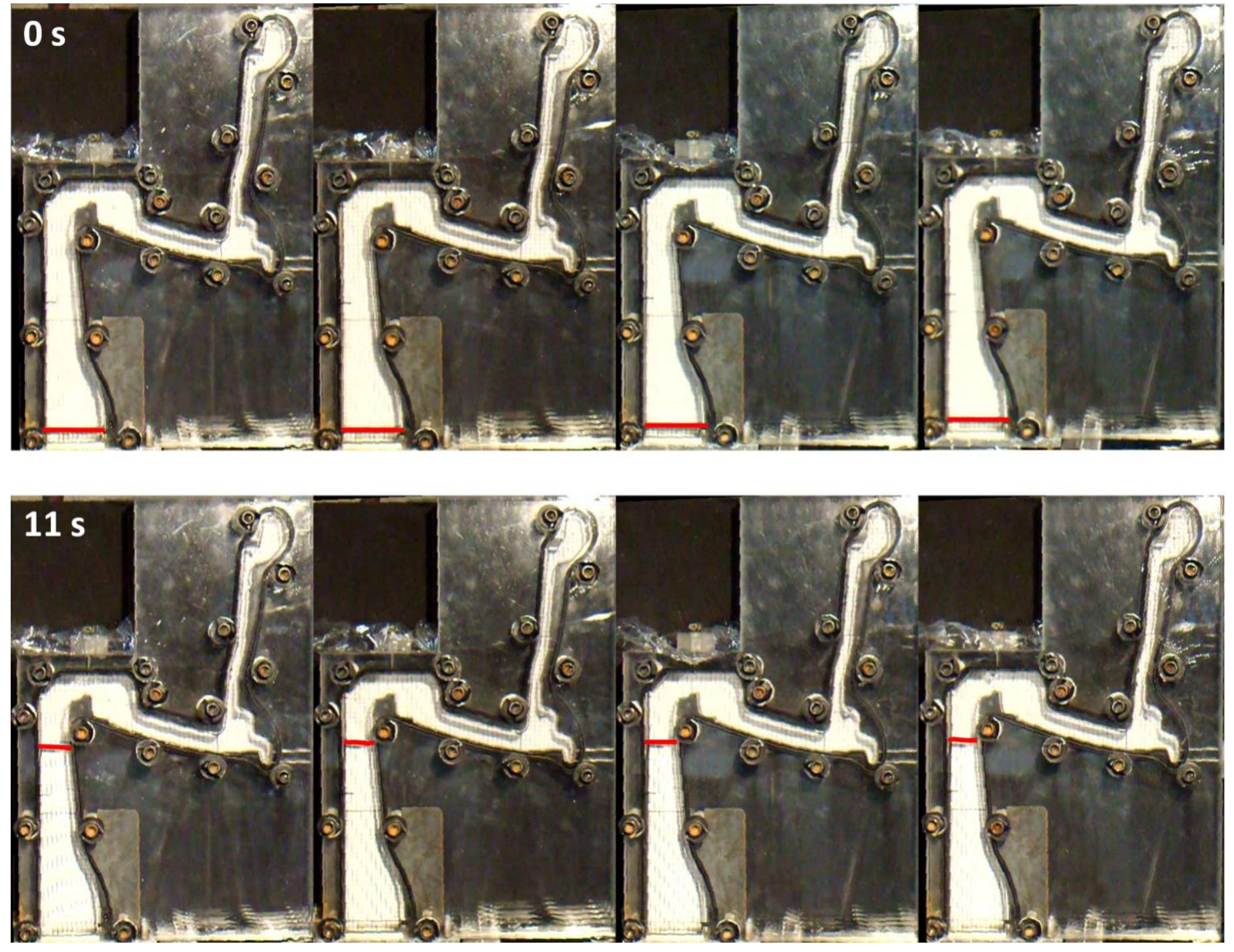
Run \#4
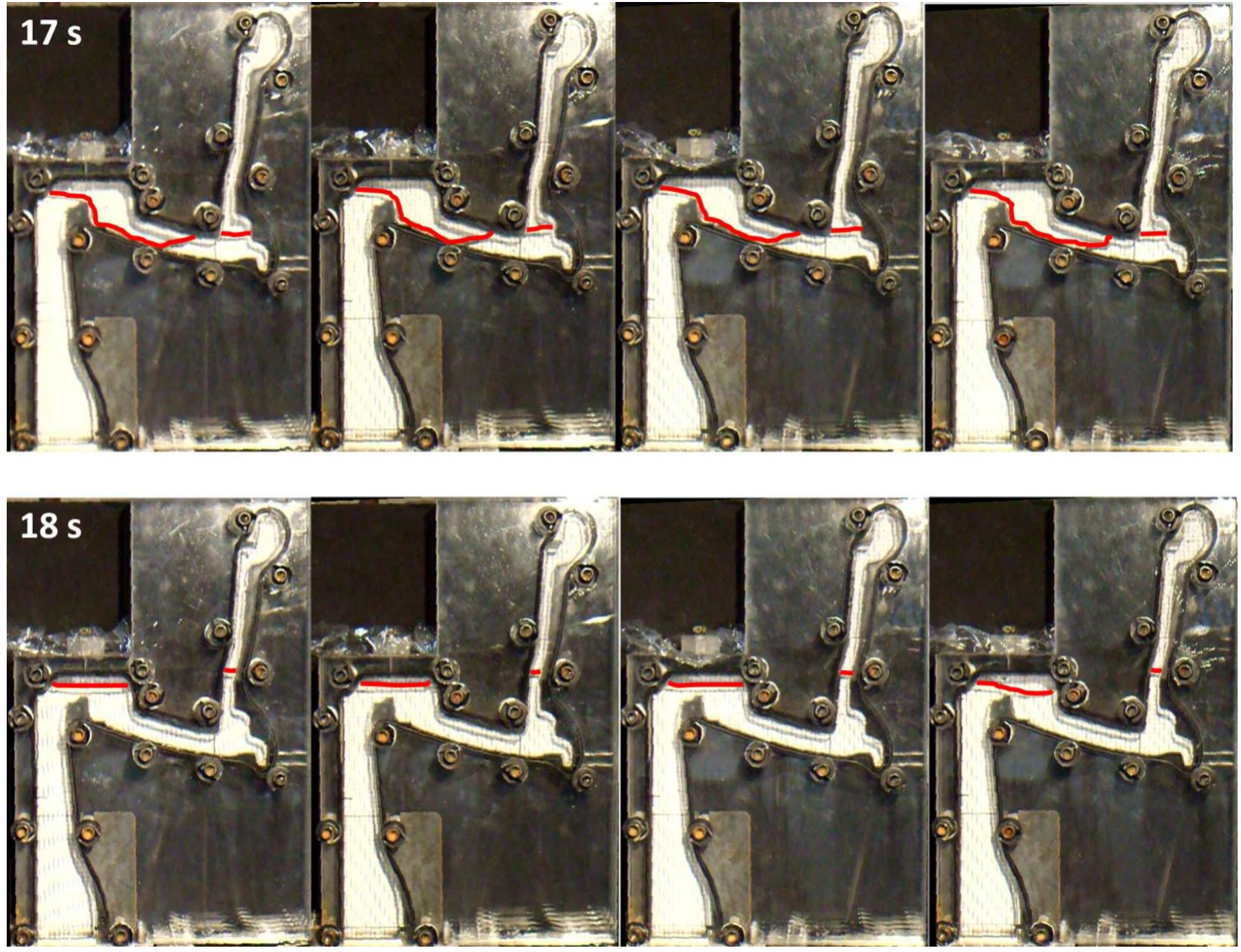
Run \#1

Run \#2

Run \#3

Run \#4

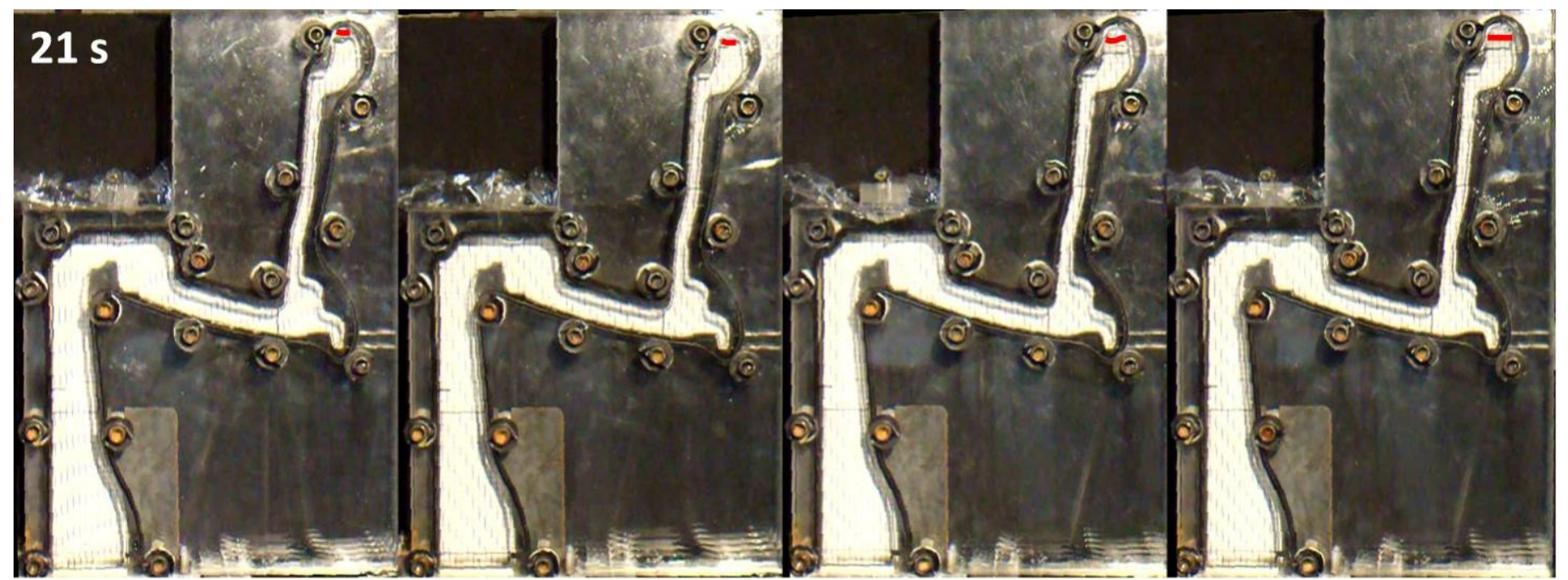

Figure 4.12. An example of the repeatability of the water analogue experiment showing four separate tests at five diffe rent time periods.(Test \#1) 


\subsubsection{Preliminary Examination to the Die Filling Process}

The fill sequence and the shapes of the free surfaces during die filling were similar for the different venting conditions. Many of them presented identical features as shown in Figure 4.13. The sprue is the first part to be filled and the flow is quiescent (Figure 4.13(a)). After filling the sprue, the water flows down to fill the outboard flange and a returning wave is generated, traveling backward from the outboard flange to fill the spoke and the hub (Figure 4.13(b)). Bubbles with diameters of $\sim 0.5-3 \mathrm{~mm}$ were seen to be entrapped below the free surface of the returning wave in this example (Figure 4.13(c)). An obvious liquid height difference between the hub and the rim was observed, with the hub not fully filling until the water had filled up to approximately half the height of the rim (Figure 4.13(d)). For Test \#1 where the hub and the riser vent are open (refer to Table 4), the whole filling process takes $21.2 \mathrm{~s}$.

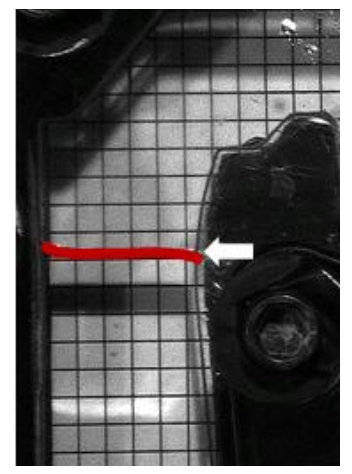

(a) Quiescent flow in the sprue

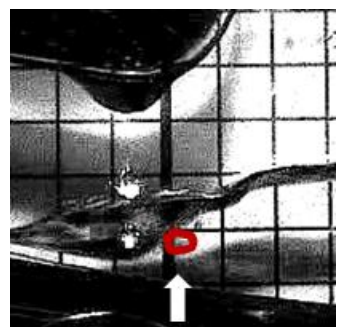

(c) Air bubbles entrained

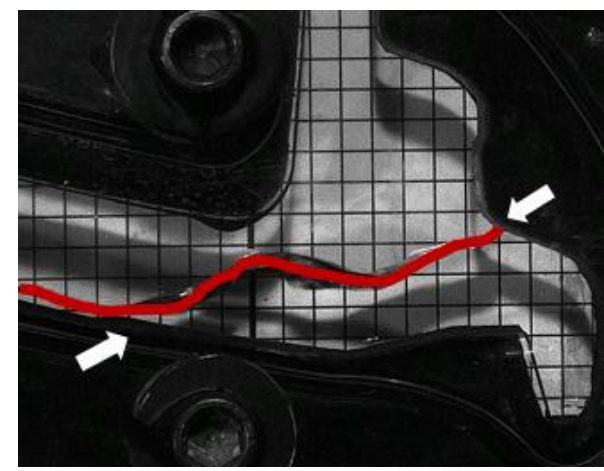

(b) Returning wave in the spoke

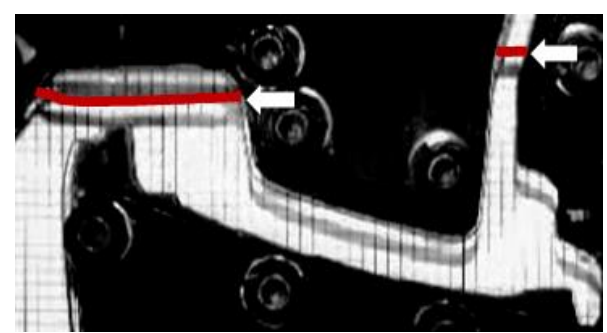

(d) Filling sequence of the hub and rim

Figure 4.13. Video images showing examples of filling behavior. Each grid square shown in the background is equal to $5 \mathrm{~mm}$ 
Detailed analysis of the effects of the different test conditions will be presented following the computational model development. 


\section{COMPUTATIONAL MODEL DEVELOPMENT}

The formation of defects related to the melt free surface in low-pressure die-cast aluminum alloy wheels is dependent on the flow conditions that occur during die filling, which include the free surface shape, localized liquid velocities, and pressure fields of both the liquid aluminum and air within the mould. However, observing the fluid flow and physically measuring the pressure and velocity at discrete locations is challenging in the casting scenario due to the inability to view the liquid metal through die walls, the complex shape of the die, the high casting temperatures, and the reactivity of liquid aluminum. Consequently, both academic researchers and foundry engineers are increasingly reliant on computational models for further refinement of the casting process.

Computational modeling has the advantage of producing both qualitative and quantitative data for the whole casting domain, at a reasonable cost. In contrast, experimentation in many cases is labor intensive, often costly and only able to offer quantitative data at a few discrete points. Despite major advances in the sophistication of commercial solidification simulation packages, the primary concern with regards to computational modeling is the accuracy of the model, i.e. how close are the calculated values to the physical realities. Therefore, model validation against either analytical solutions or experimental data is required if the simulated results are to be trusted.

In this study, a computational model was developed to reproduce the filling process of a die cavity. The model was used to assess the critical parameters that affect the flow behavior during die filling. The model was developed to simulate the water analogue experiment employed in this research project (refer to Chapter 4), using the commercial Computational Fluid Dynamics (CFD) program, ANSYS CFX version 12.0 and was validated against the data obtained 
experimentally. This section details the formulation of the model, including mesh generation, material properties, boundary conditions, solution technique, and provides preliminary results from the model.

\subsection{Filling Model Formulation}

The governing equations underpinning ANSYS CFX are a set of nonlinear partial differential equations known as the Navier-Stokes equations. The Navier-Stokes equations satisfy the conservation of mass, momentum, and energy. As the experimental program and the companion mathematical analyses are focused on an isothermal water model, the energy equation was not considered in this study. In a real casting scenarios, which are not isothermal, the energy is necessary however, as most surface oxide films are entrained into the melts before a significant degree of solidification has taken place, some aspects of their formation can be studied without inclusion of heat transport. Turbulence was also not included since it was believed that the low applied pressure would not induce sufficiently high velocities. The instantaneous equations of mass and momentum conservation can be written as follows for a stationary reference frame:

$$
\begin{array}{r}
\frac{\partial \rho}{\partial t}+\rho \nabla \cdot \vec{v}=0 \\
\frac{\partial}{\partial \mathrm{t}}(\rho \overrightarrow{\mathrm{v}})+\nabla \cdot(\rho \overrightarrow{\mathrm{v}} \overrightarrow{\mathrm{v}})=-\nabla \mathrm{p}+\mu \nabla \cdot \tau+\mathrm{S}_{\mathrm{M}}
\end{array}
$$

Equation 5.2

where $\rho$ is density $\left(\mathrm{kg} / \mathrm{m}^{3}\right), \vec{v}$ is velocity $(\mathrm{m} / \mathrm{s}), t$ is time $(\mathrm{s}), p$ is static pressure $(\mathrm{Pa}), \mu$ is viscosity $(\mathrm{Pa} \bullet \mathrm{s}), \tau$ is stress tensor $(\mathrm{Pa})$, and $S_{M}$ is a momentum source term to account for external body forces $\left(\mathrm{N} / \mathrm{m}^{3}\right)$. In the current problem the $\mathrm{N}-\mathrm{S}$ equations are solved numerically using the finite 
volume formulation. The solution of these equations provides an approximate solution for the component velocities and pressure throughout the domain.

Equation 5.1 and Equation 5.2 describe the mass and momentum balances for one phase. However, the die filling process is a multi-phase problem as it includes both the liquid entering the mould cavity and the gas being displaced from the cavity. In the present study, the model is principally applied to the water model system hence the liquid is water. In the two-phase analyses, the phases are separated by a distinct interface typically referred to as the free surface.

CFX provides the option for both a limiting case model and a full Eulerian-Eulerian multiphase model to solve for the mass, velocity and pressure fields in the domain. These are termed the homogeneous and inhomogeneous models respectively. The homogeneous model results in all fluids sharing a common flow field. It is normally used in the case where the interphase transfer rate is very large. In the inhomogeneous multiphase model, the velocities of each phase are solved for separately. Transported quantities, such as mass, momentum and energy, interact via interphase transfer terms. In this study, the inhomogeneous model was used as it allows the entrainment of one phase within the other to occur and their separation to occur.

The hydrodynamic equations for the inhomogeneous model are variants of the Navier-Stokes equations. Both volume fraction and interfacial forces were introduced into the original NavierStokes equations, as shown below:

$$
\begin{array}{r}
\frac{\partial}{\partial \mathrm{t}}\left(\gamma_{\alpha} \rho_{\alpha}\right)+\nabla \cdot\left(\gamma_{\alpha} \rho_{\alpha} \overrightarrow{\mathrm{v}_{\alpha}}\right)=0 \\
\frac{\partial}{\partial t}\left(\gamma_{\alpha} \rho_{\alpha} \overrightarrow{v_{\alpha}}\right)+\nabla \cdot\left(\gamma_{\alpha}\left(\rho_{\alpha} \overrightarrow{v_{\alpha}} \overrightarrow{v_{\alpha}}\right)=-\gamma_{\alpha} \nabla p_{\alpha}+\nabla \cdot\right. \\
\left(\gamma_{\alpha} \mu_{\alpha}\left(\nabla \overrightarrow{v_{\alpha}}+\left(\nabla \overrightarrow{v_{\alpha}}\right)^{\mathrm{T}}\right)+S_{M \alpha}+M_{\alpha}\right.
\end{array}
$$


where $\gamma_{\alpha}, \rho_{\alpha}, \vec{v}_{\alpha}, P_{\alpha}, \mu_{a}$ are the volume fraction, the density $\left(\mathrm{kg} / \mathrm{m}^{3}\right)$, the velocity $(\mathrm{m} / \mathrm{s})$, the static pressure $(\mathrm{Pa})$, and the viscosity $(\mathrm{Pa} \bullet \mathrm{s})$ of phase $\alpha$ respectively. $S_{M \alpha}$ is the momentum source term due to external body forces $\left(\mathrm{N} / \mathrm{m}^{3}\right) . M_{\alpha}$ describes the interfacial forces acting on phase $\alpha$ due to the presence of other phases $\left(\mathrm{N} / \mathrm{m}^{3}\right)$.

Volume conservation requires that the volume fractions of all phases sum to unity:

$$
\sum_{\alpha=1}^{N_{p}} \gamma_{\alpha}=1
$$

where $N_{p}$ is the total number of phases presented and $\gamma_{\alpha}$ is the volume fraction of phase $\alpha$.

The system of hydrodynamic equations is closed by the pressure constraints, namely that all phases share the same pressure field:

$$
p_{\alpha}=p \text { for all } \alpha=1, \ldots, N_{p}
$$

The solution of the above set of equations provide the component velocities of each phase $\left(U_{\alpha}\right.$, $\left.V_{\alpha}, W_{\alpha}\right)$, pressure of each phase $P_{\alpha}$, and volume fractions of each phase $\left(\gamma_{\alpha}\right)$, which enables tracking the location of free surface between fluids with time.

The double precision solver executable was used to improve convergence for free surface cases. Other solver options are variables in CFX and will not be discussed further. More information can be found in the CFX documentation [89]. A time step size of $0.01 \mathrm{~s}$ and a convergence tolerance of 0.001 for the maximum residuals were used in the simulations.

\subsection{Geometry}

The sprue and wheel cavity geometries employed in the die filling experiment were discretized in the filling model, to enable direct comparison to the water analogue experiment results. As 
discussed in Section 4.1.1.1 Pseudo Die Thickness Determination, the effects of the walls in the $18 \mathrm{~mm}$ thick experimental cavity were found to be negligible and hence it was used in the physical model experimental program. In CFX, to minimize computational time a $1 \mathrm{~mm}, 1$ element thick 3D domain was used to approximate the water model geometry. Symmetry boundary conditions were applied on the two walls perpendicular to the thickness direction. This geometry is termed quasi $2 \mathrm{D}$ in this thesis. Table 5.1 summarizes the base mesh used for the water model. All of the meshes used in the study were made with the software ANSYS GAMBIT version 2.4.

Table 5.1. Summary of Mesh Geometries

\begin{tabular}{cccc}
\hline Model & Element Type & Nodes & Elements \\
\hline Quasi 2D model & Hexahedra & 39916 & 19124 \\
\hline
\end{tabular}

The geometry of the quasi $2 \mathrm{D}$ model is shown in Figure 5.1 . The small $1 \mathrm{~mm} \times 1 \mathrm{~mm} \times 1 \mathrm{~mm}$ sub-section expansion shown in the upper insert is the vent. The methodology employed to describe the venting effect will be detailed in Section 5.5.2. The global mesh size is $1 \mathrm{~mm}$ while the vent has a refined mesh of around $0.33 \mathrm{~mm}$.

The mesh in close proximity to the walls of the domain (exemplified by the mesh at the ornament of the wheel and presented in the lower sub-section expansion in Figure 5.1) is aligned with the boundaries of the wheel geometry, and thus is very likely aligned with the direction of the flow adjacent to the boundaries. This alignment is advantageous because it substantially improves convergence. 


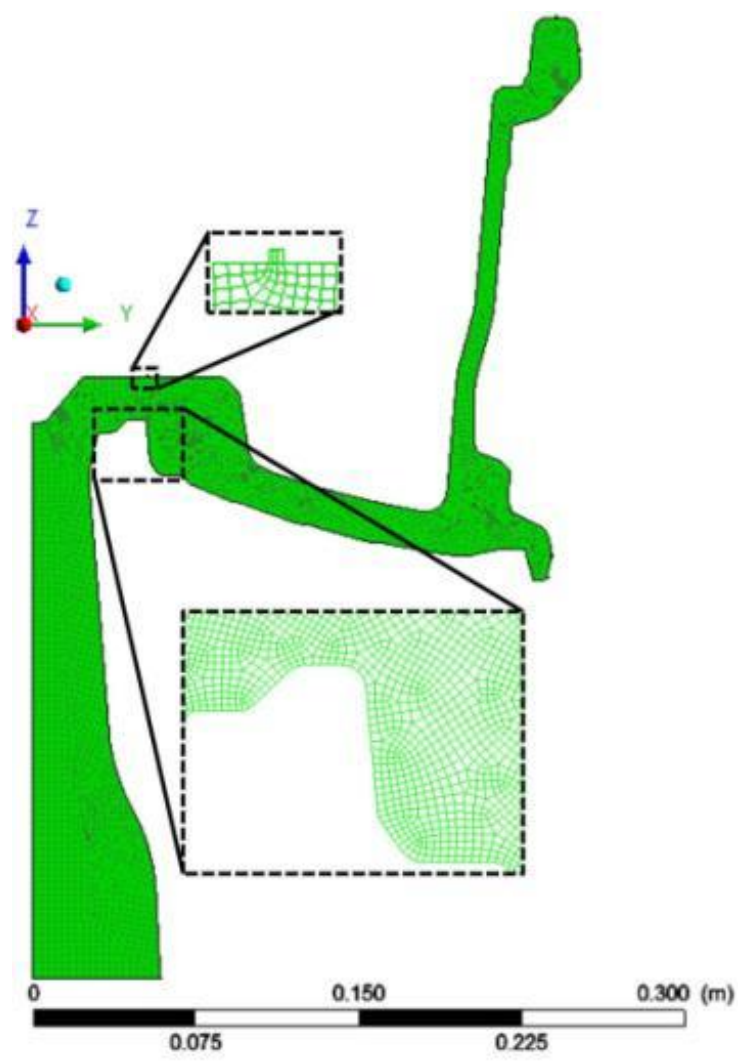

Figure 5.1. 2D mesh of sprue and wheel cavity. $1 \mathrm{~mm}^{3}$ vent can be seen in the upper close up view

\subsection{Materials Properties}

Water and air were specified as the primary and secondary fluids respectively, their properties are presented in Table 5.2. The water used in the experiment was tap water. The water density was obtained by measuring the weight and volume of a sample, using a balance with a resolution of $0.1 \mathrm{mg}$ and a burette with a resolution of $0.1 \mathrm{ml}$, respectively. The temperature of tap water in the lab was around $17{ }^{\circ} \mathrm{C}$.

Table 5.2. Fluid properties

\begin{tabular}{cccc}
\hline Fluid & $\begin{array}{c}\text { Density } \\
\left(\mathrm{kg} / \mathrm{m}^{3}\right)\end{array}$ & $\begin{array}{c}\text { Viscosity } \\
(\mathrm{kg} / \mathrm{m} \bullet \mathrm{s})[89]\end{array}$ & $\begin{array}{c}\text { Surface Tension } \\
(\mathrm{N} / \mathrm{m})[5]\end{array}$ \\
\hline Water & 997.81 & $8.899 \mathrm{e}-4$ & 0.072 \\
Air [89] & 1.185 & $1.183 \mathrm{e}-5$ & \\
\hline
\end{tabular}




\subsection{Initial Condition}

In an attempt to maintain the same initial condition for the different filling tests (refer to Section 4.2 Test Conditions), tests were started statically at an initial liquid height of $20 \mathrm{~mm}$ above the sprue entrance. This condition was reproduced in the filling model as an initial condition, as shown in Figure 5.2, where the initial velocity for both water and air was set to $0 \mathrm{~m} / \mathrm{s}$, and pressure was set to the hydrostatic pressure in the region occupied by water and $0 \mathrm{~Pa}$ everywhere else.

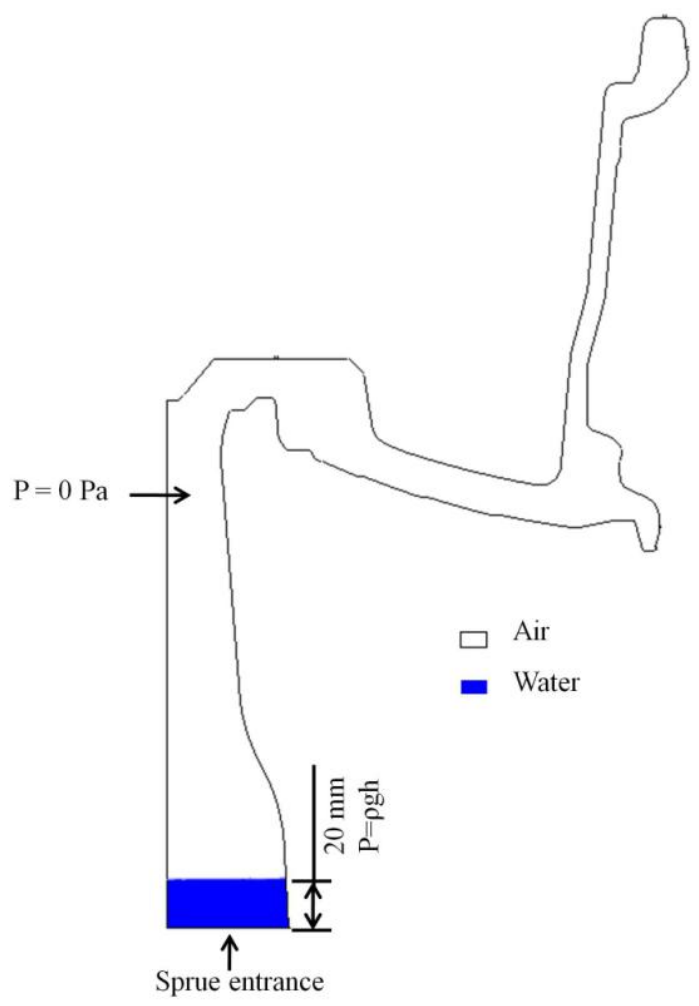

Figure 5.2. Summary of initial condition

\subsection{Boundary Conditions}

Several different types of boundary conditions were required in the formulation of the $\mathrm{d}$ ie filling model. The boundary conditions were derived from the water analogue experiment. These boundary conditions include the application of the pressure curve measured at the inlet during 
the experiments, venting, as well as other boundary conditions. These boundary conditions are summarized in Figure 5.3, and will be discussed separately in the following sections.

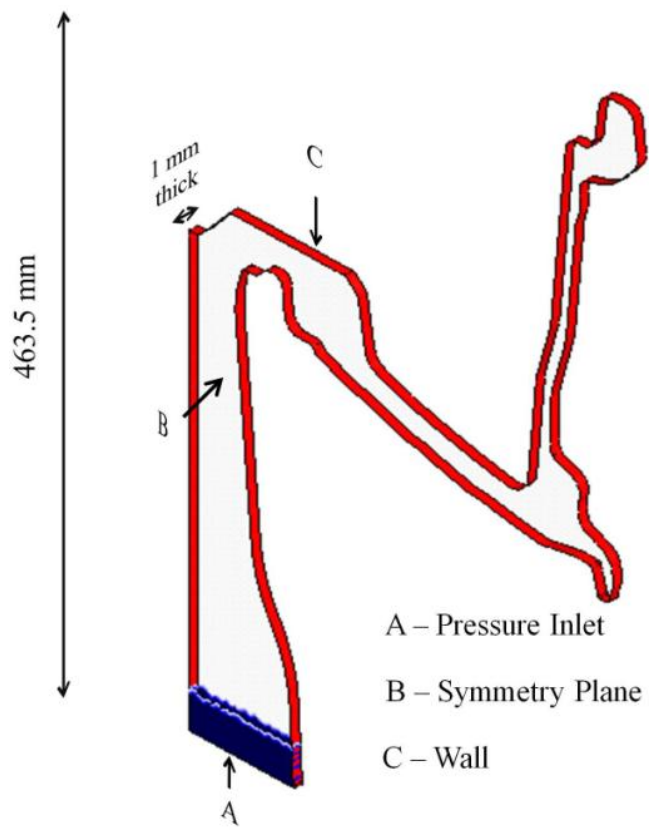

(a) Isometric vie w (the thickness dimension is magnified five times to clearly show the wall boundaries )

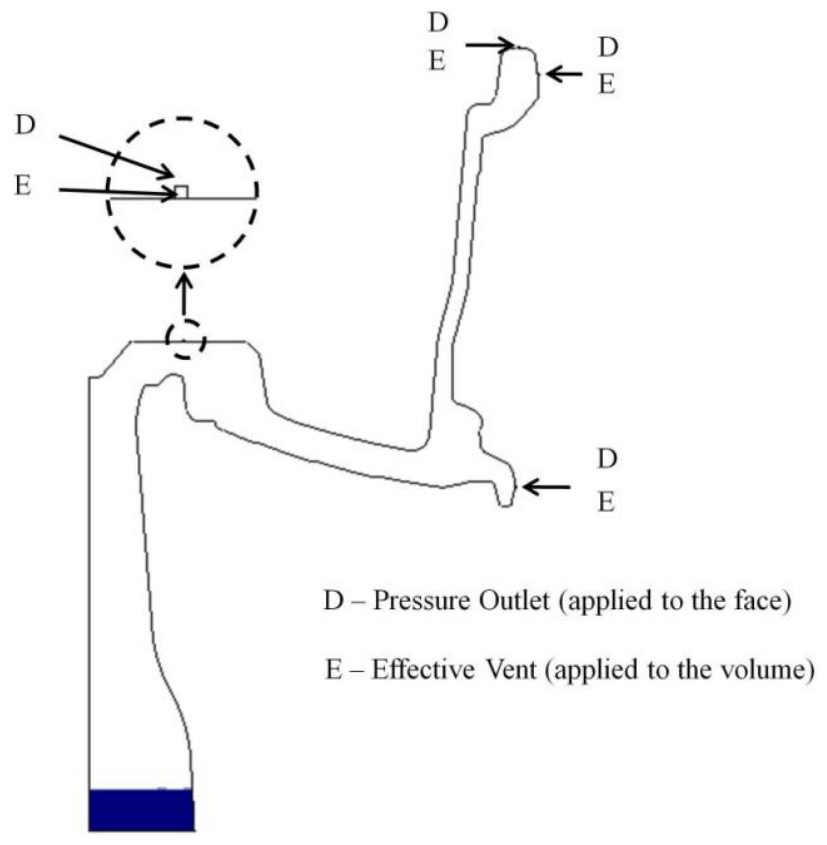

(b) Front view

Figure 5.3. Summary of boundary conditions 


\subsubsection{Inlet Pressure Boundary Condition}

A pressure inlet boundary condition was applied across the entrance to the sprue. The values for the transient pressure profile, shown in Table 5.3, were taken from the pressure curve measured at the bottom of the transition pipe (refer to Section 4.1.2 Transition Pipe Design and Section 4.1.3 Pressure Control System). The measured pressure in the experiment is the static pressure, however, the total pressure of the fluid, was applied at the inlet in the computational model. In CFX, the total pressure is defined as the sum of the static pressure and the dynamic pressure. This option is more stable than static pressure since it constrains the momentum passing through the boundary by both the transverse and the normal stresses. By choosing the option of total pressure over static pressure, the effect of the dynamic pressure in the experiment was neglected, which is expected to be minimal because of the expected low velocities during the fill process.

Table 5.3. Pressure curve data applied in the computational model, obtained from water filling expe riment. Test \#10 values presented

\begin{tabular}{cccccc}
\hline Time & $\begin{array}{c}\text { Pressure } \\
(\mathrm{Pa})\end{array}$ & Time & $\begin{array}{c}\text { Pressure } \\
(\mathrm{Pa})\end{array}$ & Time & $\begin{array}{c}\text { Pressure } \\
(\mathrm{Pa})\end{array}$ \\
\hline 0 & 238.804 & 12.31 & 2209.906 & 19.80 & 3779.906 \\
0.08 & 225.906 & 13.89 & 2459.906 & 20.07 & 4039.906 \\
0.85 & 241.906 & 14.72 & 2579.906 & 20.20 & 4079.906 \\
1.50 & 268.806 & 14.94 & 2619.906 & 20.75 & 4179.906 \\
1.75 & 284.906 & 16.90 & 2729.906 & 21.02 & 4259.906 \\
2.61 & 377.906 & 17.10 & 2736.906 & 21.36 & 4309.906 \\
5.00 & 819.906 & 18.30 & 2779.906 & 21.75 & 6199.906 \\
9.14 & 1669.906 & 18.70 & 2799.906 & & \\
10.78 & 1966.906 & 19.05 & 2839.906 & & \\
\hline
\end{tabular}


The volume fractions of water and air at the inlet were defined as 1 and 0 respectively. Therefore, only water entered the domain.

\subsubsection{Outlets}

Outlet boundary conditions were used at locations (vents) where the flow was predominantly out of the domain to compensate for the incoming fluid at the inlet and to satisfy continuity. For pressure specified boundary conditions, the option of the static pressure is typically used to describe an outlet boundary where the flow is out of the domain, and the total pressure option to specify an inlet boundary where flow is into the domain. However, in cases where a recirculation region, and therefore an inflow at the outlet is expected, CFX provides the option of a pressure specified opening boundary condition that allows for simultaneous inflow and outflow over a single region. In the filling model in this work, there is more than one vent in the geometry. While air is pushed out of the domain through one vent, it could be drawn in through the other. Thus the opening option was chosen for all outlet boundary conditions. The relative pressure to the atmosphere was $0 \mathrm{~Pa}$, and the flow direction at the openings was normal to the boundary. The volume fraction of air at the opening was set to 1 while the volume fraction of water was set to 0 . The volume fraction values are only used if there is flow into the domain over some region of the opening.

To maintain as much similarity between the computational model and the water model, an attempt was made to match the vent area-to-die cavity volume ratio of the two. One challenge with using a $1 \mathrm{~mm}$ thick geometry instead of the actual $18 \mathrm{~mm}$ geometry is that similarity would require the area of the vent in the computational model to be around $4.36 \times 10^{-2} \mathrm{~mm}, 1 / 18$ of the vent area in the experimental die cavity. This would require an impractical mesh size, resulting in an increased computation time. To address this problem, the CFD package used in the present 
study, CFX, provides an option for adding a volumetric momentum source term at locations corresponding to each vent. With this option, users can adopt a reasonable size for the vent based on considerations of the overall domain size and computational size of the problem and then tune vent performance to match the actual vent performance by providing suitable permeability and loss coefficients. The source term is comprised of a viscous loss term and an inertial loss term, and can be arranged to give the pressure loss term in the format presented in Equation 5.7.

$$
\Delta p=\mathrm{l} \cdot\left(\frac{\mu}{K_{\mathrm{perm}}} u-K_{\text {loss }} \frac{\rho}{2} u^{2}\right)
$$

where $\Delta p$ is the pressure drop over the vent $(\mathrm{Pa}), l$ is the length of the vent $(\mathrm{m}), \mu$ is the viscosity of air $(\mathrm{Pa} \bullet \mathrm{s}), K_{\text {pem }}$ is the permeability of the vent $\left(\mathrm{m}^{2}\right), K_{\text {loss }}$ is the loss coefficient of the vent $\left(\mathrm{m}^{-}\right.$ $\left.{ }^{1}\right), \rho$ is the air density $\left(\mathrm{kg} / \mathrm{m}^{3}\right), u$ is the air velocity through the vent $(\mathrm{m} / \mathrm{s})$. The linear component of this source term represents the viscous losses and the quadratic term represents the inertial losses.

\subsubsection{Vent Resistance Coefficients Determination}

The vent geometry in the experimental water model cavity is comprised of two sections - a $\phi 1 \mathrm{~mm}$ section connected to the die cavity and a larger $\phi 2 \mathrm{~mm}$ section, connecting the small diameter-section to the ambient air, as shown in the left picture of Figure 5.4. Each section is estimated to be $20 \mathrm{~mm}$ long. The vent geometry adopted in the computational model was a $1 \mathrm{~mm} \times 1 \mathrm{~mm} \times 1 \mathrm{~mm}$ block, as shown in the right picture of Figure 5.4. Permeability and loss coefficients were applied to the volume of the block to maintain the same pressure drop over the domain. The degree to which this mimics the venting in the actual industrial die is difficult to assess owing to the challenge in characterizing the industrial die venting area. 

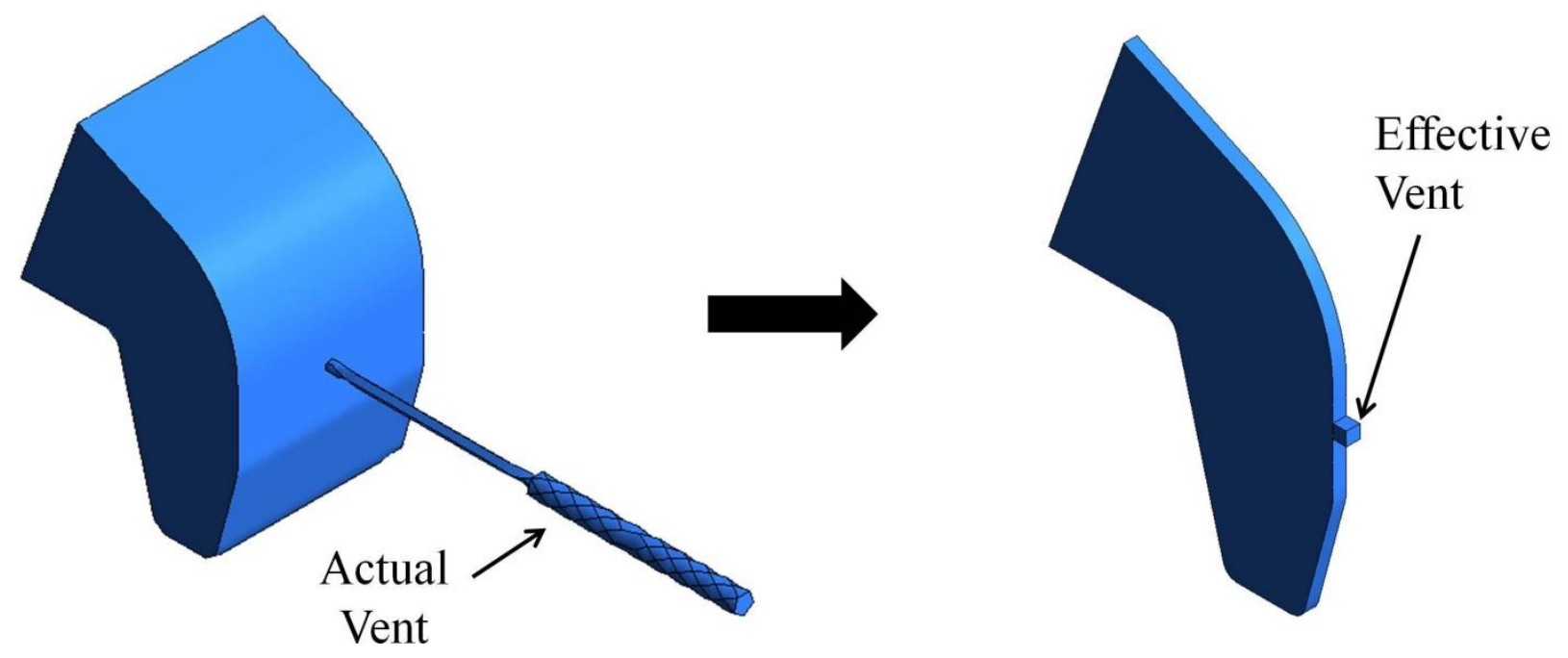

Figure 5.4. Schematics of the methodology employed to effectively address venting in CFX

The process of obtaining the values for permeability and loss coefficients was as follows. The geometry of the vent from the experimental apparatus was used to characterize the pressure drop of air flowing through the vents. This geometry also includes a sub-domain of the die cavity. The relationship between the pressure drop and the velocity through the vent was then obtained. The experimental vent geometry has 32222 tetrahedra at the size of approximately $1 \mathrm{~mm}$ in length, as shown in Figure 5.5.

A steady-state, single phase, laminar flow model was used for this simulation. Gravity was defined to apply in the negative $\mathrm{z}$ direction. The fluid was defined as air with constant properties. The initial velocity and static pressure in the domain was set to 0 . A specified velocity inlet boundary condition was applied to the wall section that represents the edge of the sub-domain (represented by multiple rightwards arrows on the left of Figure 5.5). A pressure-specified opening boundary condition was applied at the end of the vent (single left right arrow on the right). All remaining walls were no-slip. 


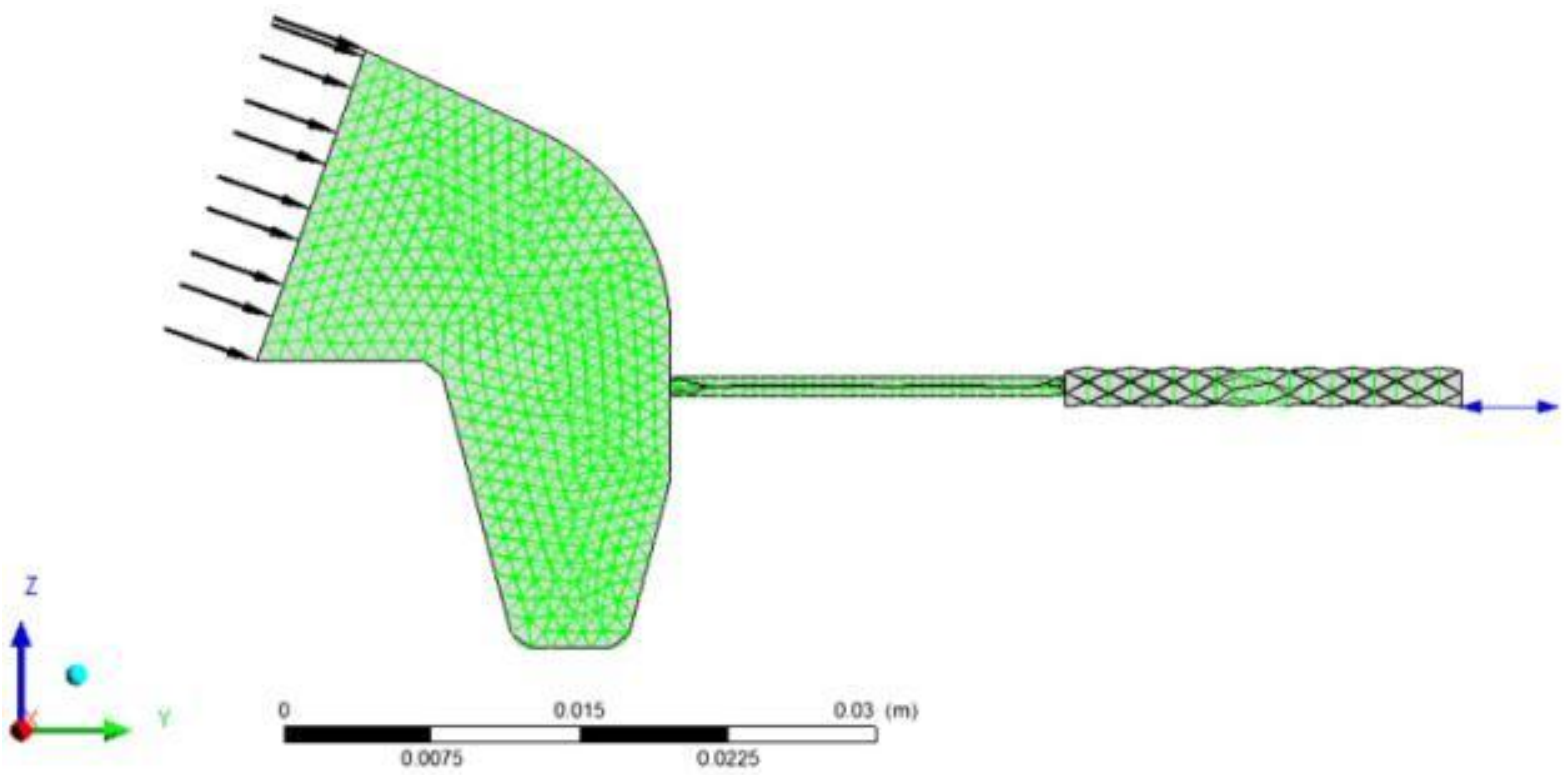

(a) Side vie w of the meshed model to replicate the physical vent geometry in the die

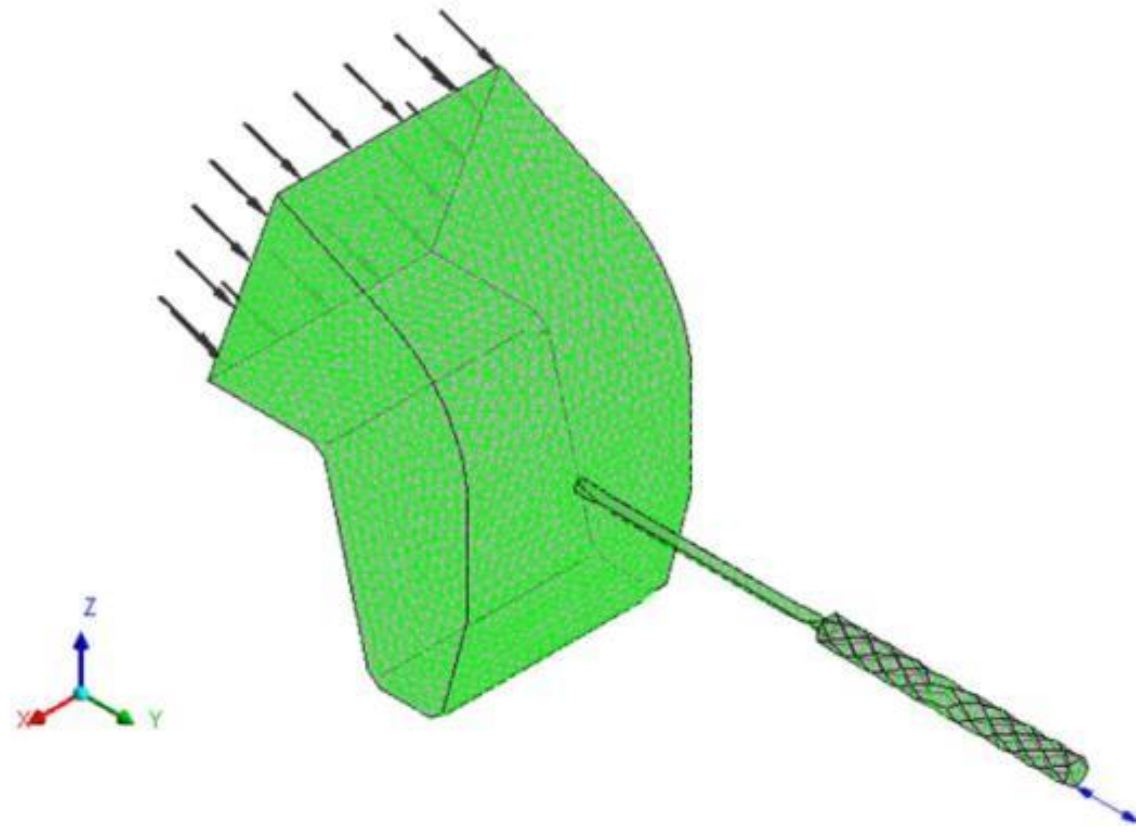

(b) Isometric view of the meshed model to replicate the physical vent geometry in the die

Figure 5.5. Vent geometry in the die cavity with the outboard flange rim included 
The velocity at the inlet was estimated to be $2.91 \times 10^{-2} \mathrm{~m} / \mathrm{s}$, based on the total volume of the die cavity, the filling time, the area of the inlet face, the number of vents and the area of the vents. A few points around this value, ranging from $0.004 \mathrm{~m} / \mathrm{s}$ to $0.03 \mathrm{~m} / \mathrm{s}$, were selected and applied to the inlet boundary. The differences between the pressure at the inlet and the pressure at the outlet were calculated and related to the corresponding average velocities through the vents through regression analysis. The results shown in Figure 5.6 follow a quadratic relationship as expected from Equation 5.7.

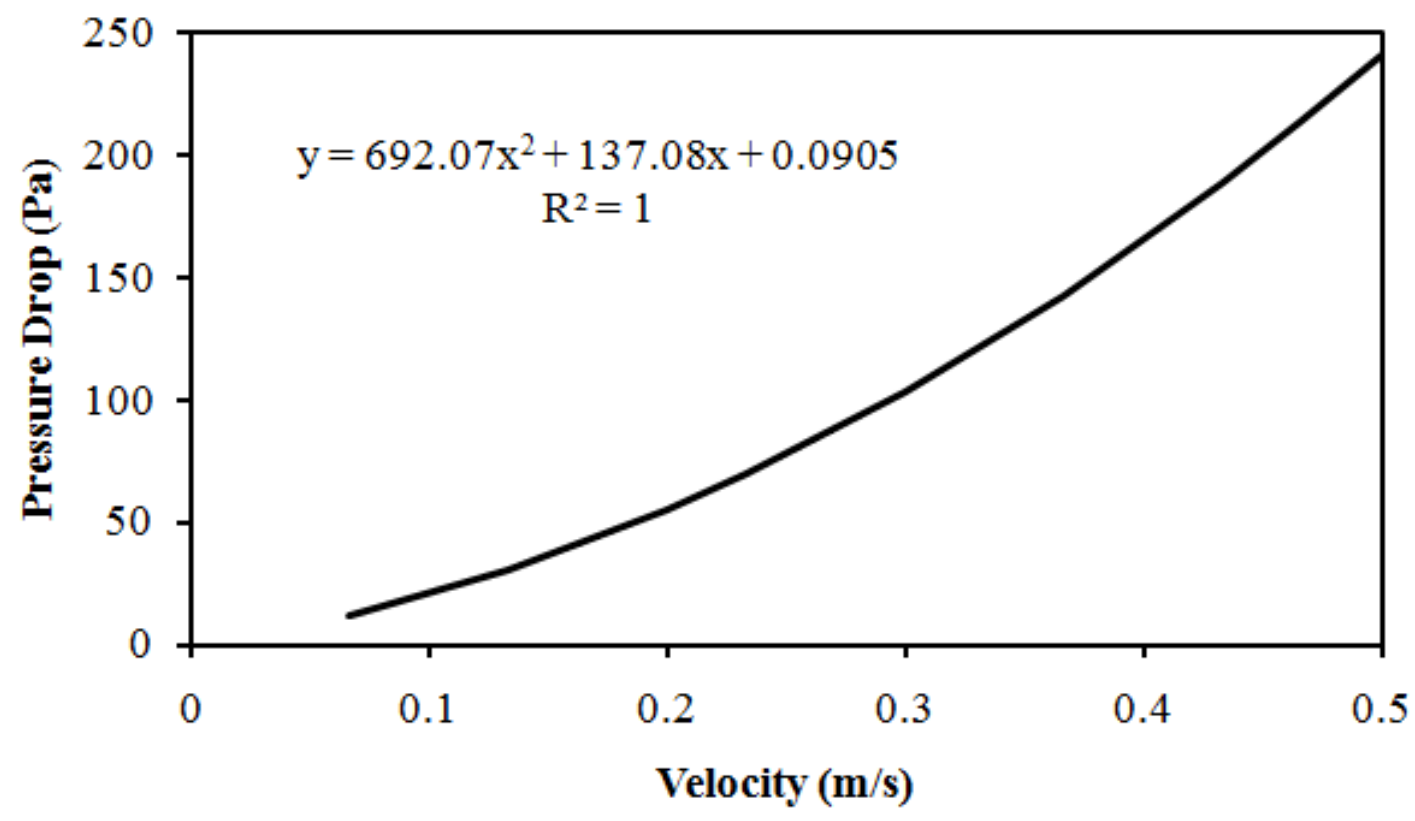

Figure 5.6. The quadratic relationship between pressure drop and the corres ponding averaged vent velocity

Comparing the correlation equation to Equation 5.7, and neglecting the constant term, the following equations were defined:

$$
l \cdot \frac{\mu}{K_{\text {perm }}}=137.08
$$




$$
\mathrm{l} \cdot K_{\text {loss }} \cdot \frac{\rho}{2}=692.07
$$

The length of the vent $l$ is $1 \mathrm{~mm}$, which yields $1.34 \times 10^{-10} \mathrm{~m}^{2}$ for $\mathrm{K}_{\text {perm }}$ and $1168051 \mathrm{~m}^{-1}$ for $\mathrm{K}_{\text {loss. }}$ These values were applied with Equation 5.7 to define the pressure loss at the vents.

\subsubsection{Symmetry and Wall Boundaries}

As illustrated in Figure 5.3 (a), symmetry boundary conditions were applied to the two side walls, which represent the middle of the flow channel. The symme try plane boundary condition imposes constraints that the flow on one side of the plane is the mirror image of flow on the opposite side. The normal component of any variables is set to zero at the symmetry plane boundary, for example, the normal velocity component.

The non-adhesive no slip condition was applied to all wall boundaries - refer to Figure 5.3 (a). Without adhesion, there is no curvature at the liquid-wall contact, which means that the contact angle between the liquid free surface and wall is $90^{\circ}$ when in static state. With the no slip condition the fluid adheres to the wall with zero velocity, creating a boundary layer along the wall.

\subsection{Verification of the Model}

In order to verify the model's ability to describe the two-phase filling process, the model was first used to analyze a simplified problem with an analytical solution. Dr Jindong Zhu, an Adjunct Professor in the casting research group in the Department of Materials Engineering, $\mathrm{UBC}$, conceived of the problem and derived the analytical solution for it. In this section, the evolution of air pressure obtained from the computational model applied to the same simplified 
problem was obtained and then compared to analytical solution. The derivation of the analytical solution is shown below.

\subsubsection{Analytical Solution}

Figure 5.7 shows a schematic diagram of the simplified problem. The fluid, presented in red, comes into the domain from the left at a constant velocity. The region to the right, appearing as white, initially contains air, which is displaced by the increasing volume of liquid. Vents are located at the right side of the domain. Gravity was neglected in this study. The mass conservation of gas leads to the following equation:

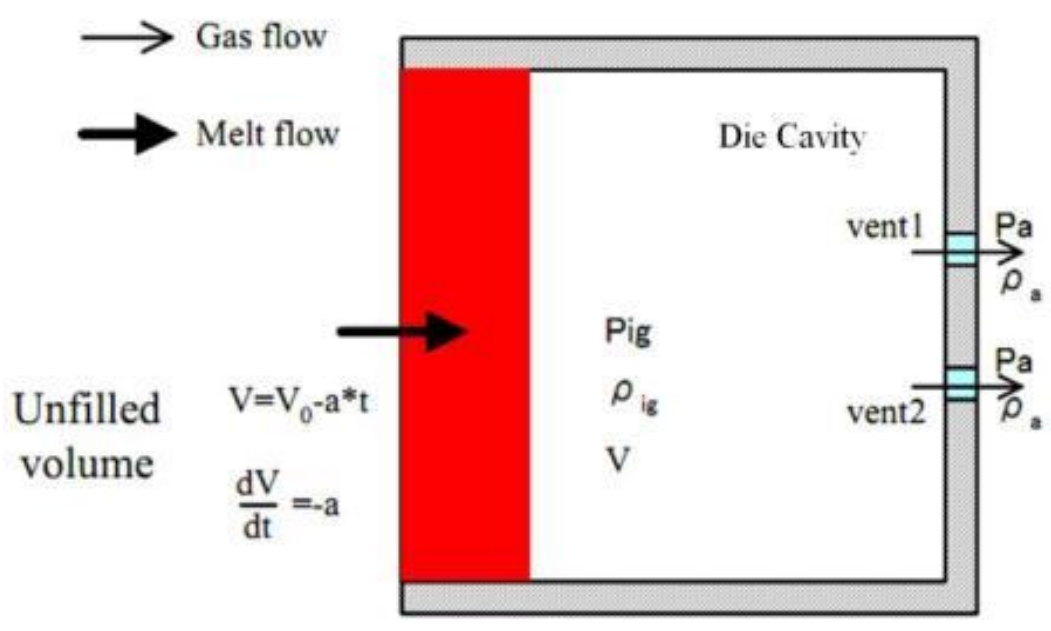

Figure 5.7. Illustration of the simple case used to evaluate backpressure simulation (courtesy Dr.

Jingdong Zhu)

$$
\frac{d\left(\rho_{i g} V\right)}{d t}=-\sum_{j} u_{j} S \rho_{i g}
$$

where $\rho_{i g}$ is the density of the remaining gas in the die cavity during filling $\left(\mathrm{kg} / \mathrm{m}^{3}\right), V$ is the unfilled volume $\left(\mathrm{m}^{3}\right), u_{j}$ is the gas velocity through the vent $(\mathrm{m} / \mathrm{s}), S$ is the area of the vent $\left(\mathrm{m}^{2}\right), \mathrm{j}$ is the vent number and ${ }_{\text {ig }}$ ind icates the remaining air in the die cavity. 
The gas velocity through the vent $u_{j}$ is proportional to the pressure drop across the vent under a laminar flow condition:

$$
\mathrm{u}_{\mathrm{j}}=\mathrm{K}\left(\mathrm{p}_{\mathrm{ig}}-\mathrm{p}_{\mathrm{a}}\right)=\frac{\mathrm{KRT}}{\mathrm{M}}\left(\rho_{\mathrm{ig}}-\rho_{\mathrm{a}}\right)
$$

where $p=\frac{\rho}{M} R T$ which is the ideal gas law, $u_{j}$ is the gas velocity through the vent $(\mathrm{m} / \mathrm{s}), K$ is the permeability of air vent $(\mathrm{m} / \mathrm{s} \bullet \mathrm{Pa}), p_{i g}$ is the backpressure $(\mathrm{Pa}), p_{a}$ is the boundary pressure of the vent $(\mathrm{Pa}), R$ is the gas constant $(\mathrm{J} / \mathrm{K} \cdot \mathrm{mol}), T$ is the temperature $(\mathrm{K}), M$ is the molar mass of gas $(\mathrm{kg} / \mathrm{kmol}), \rho_{i g}$ is the density of the remaining gas in the die cavity during filling $\left(\mathrm{kg} / \mathrm{m}^{3}\right)$, and $\rho_{a}$ is the gas density corresponding to boundary pressure $\left(\mathrm{kg} / \mathrm{m}^{3}\right)$.

By substituting Equation 5.11 into Equation 5.10, and then applying the following initial and boundary conditions, an exact solution describing the evolution of backpressure in the die cavity with time was obtained:

$$
\begin{array}{r}
V=V_{0}-a t, \quad a=u_{i n} S_{i n} \\
\rho_{i g}^{0}=\rho_{0}=\rho_{a} \\
p_{i g}^{0}=p_{0}=p_{a} \\
T_{i g}=T_{0}=T_{a}
\end{array}
$$

Equation 5.15 


$$
\begin{gathered}
\mathrm{p}_{\mathrm{j}}=\frac{\mathrm{RT}}{\mathrm{M}}\left(\frac{1+\frac{\mathrm{RT} \sum \mathrm{KS}}{\mathrm{a}} \rho_{\mathrm{a}}}{\frac{\mathrm{RT} \sum \mathrm{KS}}{\mathrm{a}}+\frac{1}{\rho_{0}}\left(1-\frac{\mathrm{at}}{\mathrm{V}_{0}}\right)^{\left(1+\frac{\mathrm{RT} \sum \mathrm{KS}}{\mathrm{a}} \rho_{\mathrm{a}}\right)}}\right) \\
=\frac{\mathrm{RT}}{\mathrm{M}}\left(\frac{1+\rho_{\mathrm{a}} \mathrm{C}}{\left.\mathrm{C}+\frac{1}{\rho_{0}}\left(1-\frac{\mathrm{at}}{\mathrm{V}_{0}}\right)^{\left(1+\rho_{\mathrm{a}} \mathrm{C}\right)}\right)}\right. \\
C=\frac{R T \sum K S}{\mathrm{a}}
\end{gathered}
$$

where $\mathrm{V}_{0}$ is the initial cavity volume $\left(\mathrm{m}^{3}\right)$, a is the volume increment constant which is the product of the die inlet area and the inlet velocity, and $\rho_{0}$ is the initial density of the air in the die cavity $\left(\mathrm{kg} / \mathrm{m}^{3}\right)$.

\subsubsection{Implementation of the Model}

The flow model developed for the simplified problem has a constant inlet velocity and is assumed isothermal, thus buoyancy force does not influence the fluid flow and was therefore not included in the study. Due to the simplicity of the die cavity, no phase entrainment and subsequent separation was expected, so the homogeneous model was used. A surface tension force was included to account for the interaction between the fluid and gas. Standard free surface option was chosen to keep the liquid-air interface clearly defined. The inlet fluid was expected to advance steadily with a uniform (vertical) free surface, so air pressure everywhere in the unfilled domain should be the same. The simulation was run for $0.2 \mathrm{~s}$ at the time step size of $0.0005 \mathrm{~s}$ and an output frequency of 1 data per $0.005 \mathrm{~s}$. Each time step has a maximum 20 iterations and the convergence target for all variables were for the maximum residual to be lower than 0.001 . Other solver options were set to be the same as in the filling model (refer to Section 5) 


\subsubsection{Geometry}

A quasi 2D mesh, $1100 \mathrm{~mm}$ long $\times 200 \mathrm{~mm}$ wide $\times 1 \mathrm{~mm}$ (1 element) thick, was developed for the model of the simplified problem. A $1 \mathrm{~mm} \times 1 \mathrm{~mm} \times 1 \mathrm{~mm}$ block was added at the top (Figure 5.8) to represent the vent in the same way described in Section 5.5.2.1. The average element edge length is $1 \mathrm{~mm}$, with the mesh at the vent refined to be $0.2 \mathrm{~mm}$ and the largest cell in the domain having an edge length of around $3.15 \mathrm{~mm}$. The mesh size change is gradual to eliminate large length aspect ratios for cells and ensure a good mesh quality. There were 453684 nodes and 225525 hexahedra elements in the geometry.

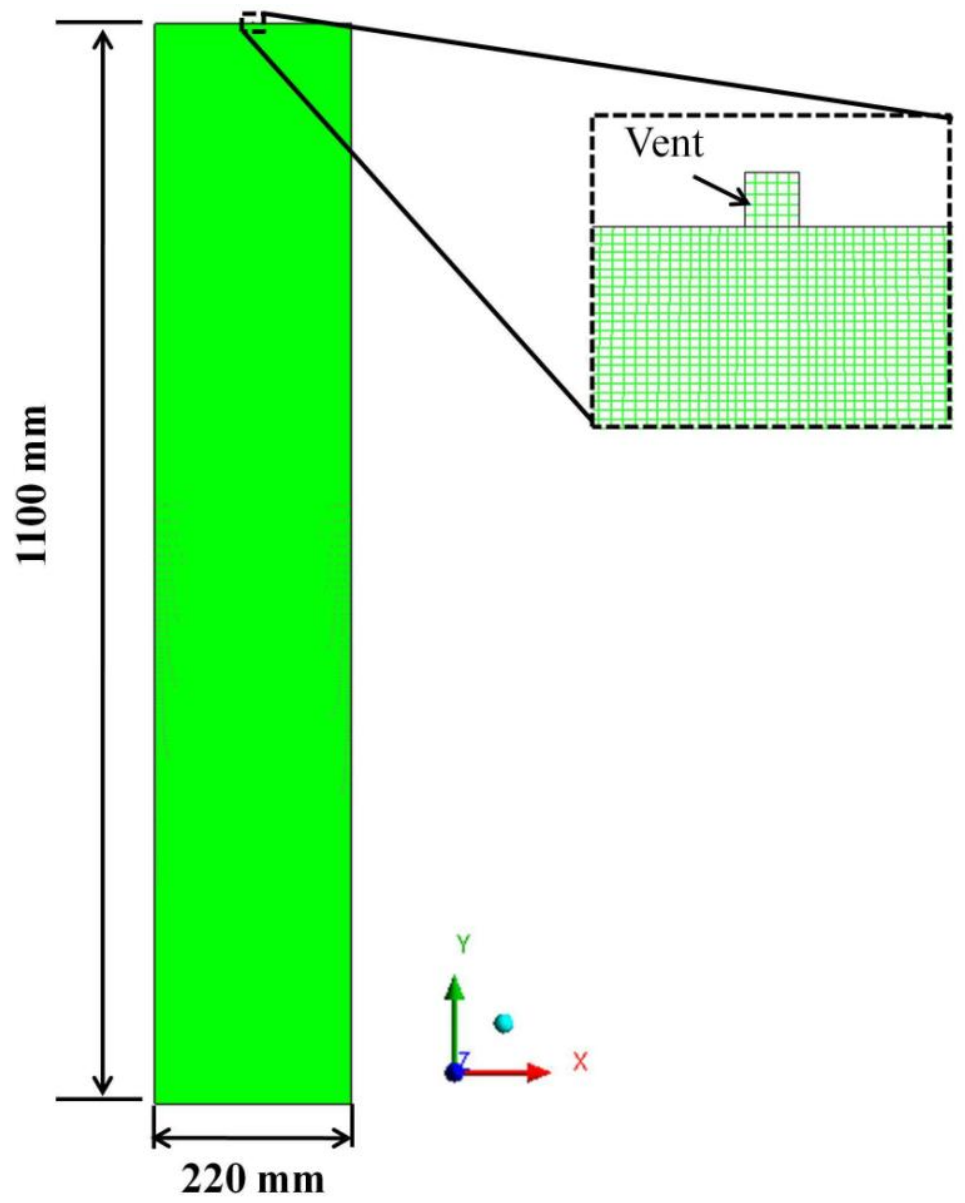

Figure 5.8. Quasi 2D mesh of the $1100 \mathrm{~mm} \times 200 \mathrm{~mm}$ model domain use d as validation against the analytical solution (courtesy Dr. Jingdong Zhu) 


\subsubsection{Material Prope rties}

Water and air were specified as the primary and secondary fluids respectively. The properties of water are the same as used in the filling model (refer to Section 5.3). Air was assumed to conform to the ideal gas law, which means the fluid is compressible.

\subsubsection{Initial and Boundary Conditions}

The domain was initially filled with stagnant air at atmospheric pressure. Water enters the domain at $5 \mathrm{~m} / \mathrm{s}$ via a velocity $\mathrm{BC}$ along the bottom surface. A pressure-specified boundary condition was applied to the top surface of the vent, and the pressure was kept at atmospheric pressure. The front and back planes perpendicular to the thickness direction were symmetry planes. The remaining walls were set to be non-adhesive, no slip boundary condition.

In the analytical solution, the inertial momentum loss for the air flowing through the vent was not included and only the viscous loss was considered. The same condition was applied to the vent in the model of the simplified problem by setting the resistance loss coefficient (refer to Section 5.5.2.1) to 0 in CFX. The relationships between the pressure drop and the viscous dissipation term in the analytical solution and CFX can then be written in the form of Equation 5.17 and Equation 5.18 respectively.

$$
\begin{gathered}
\Delta p=\frac{u}{\mathrm{~K}_{A n a}} \\
\Delta p=\frac{l \cdot \mu}{K_{\mathrm{CFX}}} u
\end{gathered}
$$


where $u$ is the velocity of air through the vent $(\mathrm{m} / \mathrm{s}), \mu$ is the viscosity of air $(\mathrm{kg} / \mathrm{m} \bullet \mathrm{s}), l$ is the length of the vent $(\mathrm{m}), K_{A n a}$ is the permeability used in analytical solution $(\mathrm{m} / \mathrm{s} \bullet \mathrm{Pa})$, and $K_{C F X}$ is the permeability applied to the model of the case-study $\left(\mathrm{m}^{2}\right)$.

To achieve the same pressure drop between the analytical case and the CFX model, Equation 5.17 was equated to Equation 5.18, and the value of the permeability $\mathrm{K}_{\mathrm{CFX}}$ can be obtained through the following equation:

$$
K_{\mathrm{CFX}}=\mathrm{K}_{\text {Ana }} \cdot l \cdot \mu
$$

The permeability given in the analytical solution is 0.173 , which yields $0.137 \times 10^{-8}\left(\mathrm{~m}^{2}\right)$ for the model.

\subsubsection{Comparison to Analytical Solution}

The model took 21.5 hours to run on four Intel Xeon $2.33 \mathrm{GHz}$ processors. The air/water interface predicted by the model for the simplified problem is shown in Figure 5.9 and is quiescent and flat as expected. The air/water interface is curved near the wall due to the effect of surface tension. At an inlet velocity of $5 \mathrm{~m} / \mathrm{s}$, the cavity is $90 \%$ full at $0.2 \mathrm{~s}$, which agrees with the calculation assuming mass conservation.

A point, shown as the grey ' + ' symbol in Figure 5.9, was selected to evaluate and compare the results for pressure variation with time predicted by the model with the analytical solution. The parameters used in the analytical solution are listed in Table 5.4. The results of the comparison appear in Figure 5.10. The solid line represents the analytical solution and the discrete squares are the numerical results obtained from $\mathrm{CFX}$ at $0.005 \mathrm{~s}$ intervals. 
Qualitatively, both the analytical and numerical results indicate that backpressure increases exponentially with the time for the conditions present in the simplified problem. Quantitatively, the pressures predicted by the model at a given time are very close to those calculated by the analytical solution. The discrepancy between the two curves is likely due to numerical errors resulted from the quality of mesh. More than $50 \%$ of the cells in the domain have a width to length ratio $1 / 3$. It is expected that decreasing the mesh length ratio could be used to improve the result. However, the current results of model satisfy the intent of verifying the modeling technique. Thus, the numerical settings applied in the simplified model can be used as a starting point in the analysis of the more complex die filling water model.

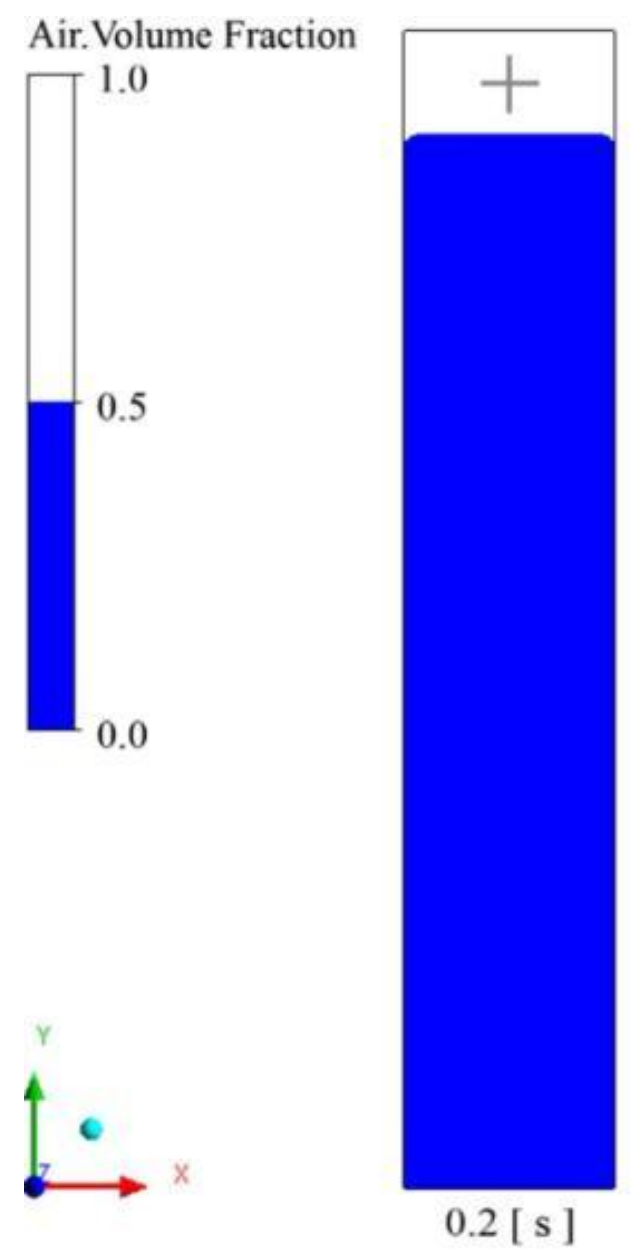

Figure 5.9. The filling state at $0.2 \mathrm{~s}$ when the die cavity is $90 \%$ full 
Table 5.4. Parameters and the corres ponding values used in the analytical solution

\begin{tabular}{ccc}
\hline Parameter & Value & Unit \\
\hline Gas constant, $R$ & 8.314 & $\mathrm{~J} / \mathrm{K} \cdot \mathrm{mol}$ \\
Temperature, $T$ & 298.15 & $\mathrm{~K}$ \\
Molar mass of air, $M$ & 0.02896 & $\mathrm{~kg} / \mathrm{mol}$ \\
Inlet area, $S_{\text {in }}$ & 0.2 & $\mathrm{~m}^{2}$ \\
Area of the vent, $S$ & 0.000001 & $\mathrm{~m}^{2}$ \\
Initial cavity volume, $V_{0}$ & 0.22 & $\mathrm{~m}^{3}$ \\
Initial density of air, $\rho_{0}$ & 1.185 & $\mathrm{~kg} / \mathrm{m}^{3}$ \\
Air density at outlet, $\rho_{a}$ & 1.185 & $\mathrm{~kg} / \mathrm{m}^{3}$ \\
Inlet velocity, $u_{\text {in }}$ & 5 & $\mathrm{~m} / \mathrm{s}$ \\
Permeability, $K$ & 0.173 & $\mathrm{~m}^{2}$ \\
\hline
\end{tabular}

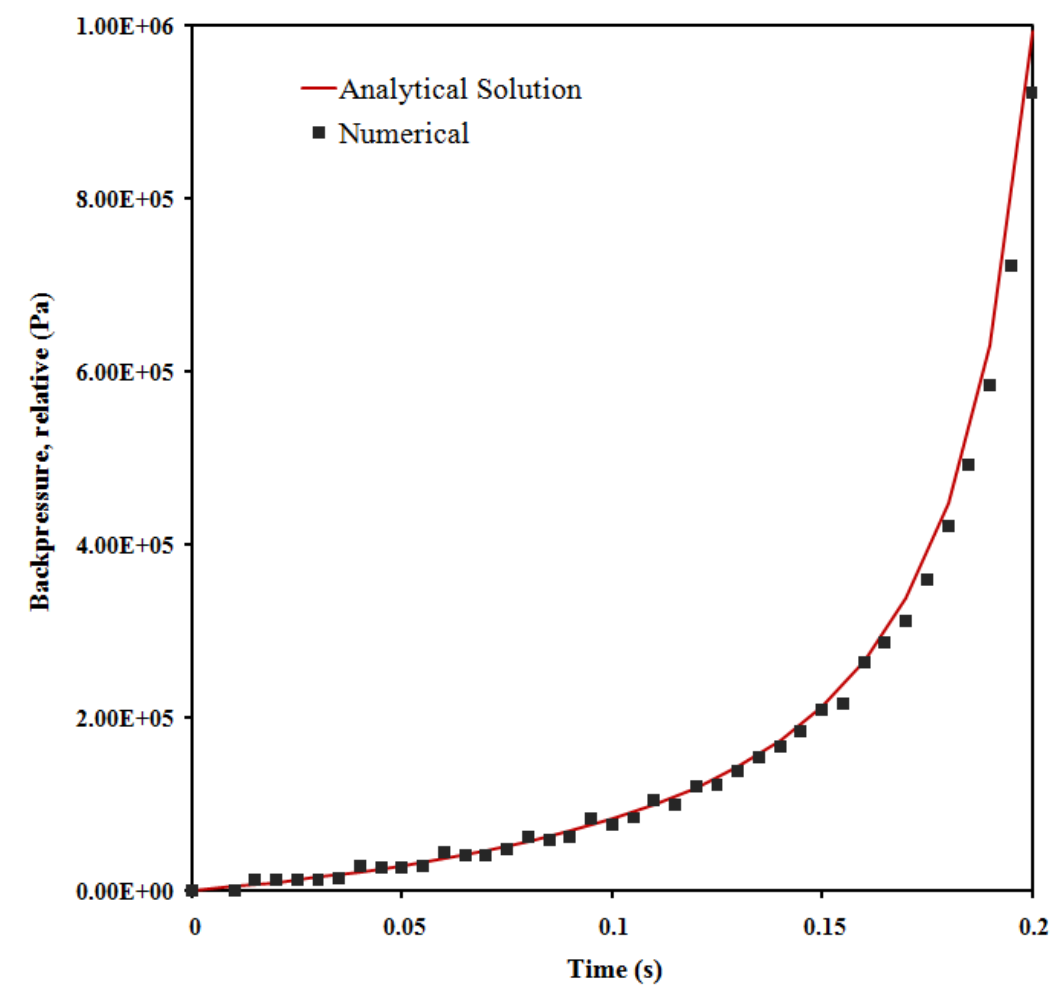

Figure 5.10. Comparison be tween nume rical and analytical solutions 


\subsection{Preliminary LP Die Computational model Results}

In this section, the results of the numerical analysis of the LP die analogue water model are discussed. Presented here are the results of the model simulating Test \#1 (refer to Chpater 4, Section 4.2 Test Conditions). The simulation time for the model is approximately 25 hours when running on two Intel Xeon processors at $2.33 \mathrm{GHz}$.

The internal velocity and pressure fields were plotted at four discrete times $-11 \mathrm{~s}, 17 \mathrm{~s}, 18 \mathrm{~s}$ and $21 \mathrm{~s}$. At these times, the free surface is at different locations - e.g. the sprue, spoke, rim and outboard flange. The data from the model can be used to analyze the free surface and the potential for air entrainment. Qualitative characteristics such as free surface shape and the liquid level have been considered, since they correlate to free surface related defects and casting quality. Also, these quantities can be compared to measurements made on the physical model.

A series of contour plots illustrating the static pressure at various times during filling is shown in Figure 5.11. Vector plots illustrating the velocity magnitude and direction at various times during filling are shown in Figure 5.12. In each plot, the results have been blanked for areas with air volume fraction larger than 0.5 to only show the results for the water phase.

The sequence of results in Figure 5.11 and Figure 5.12 show that the sprue is the first region to be filled. The fluid then flows over the ornament area (refer to Figure 1.5 in Chapter 1) and down along the spoke to fill the outboard flange (Figure 5.11 (a) and (b), Figure 5.12 (a) and (b)). As the spoke and hub are filled, a returning wave is generated by the fluid reflection at the outboard flange (Figure 5.11 (c) and (d), Figure 5.12 (c) and (d)). An obvious liquid height difference between the hub and the rim was observed during filling. However, the hub and the rim are completely filled at nearly the same time (Figure 5.11 (d), Figure 5.12 (d)). 
Pressure is highest at the sprue entrance where fluid enters the domain. With increasing time, the pressure increases according to the pressure profile described in Section 5.5.1. The static pressure of the water increases with depth, which obeys Pascal's Law. The pressure contours are generally parallel to the sprue entrance.

One interesting phenomenon shown in Figure 5.11 is that before the liquid starts to fill the hub, the pressure at the free surface is close to $0 \mathrm{~Pa}$; however, it increases to $\sim 1500 \mathrm{~Pa}$ in the hub at $21 \mathrm{~s}$. The pressure in the hub area is also slightly higher than the pressure in the rim (Figure $5.11(\mathrm{~d}))$. This phenomenon indicates that appreciable backpressure in the air is not generated until the hub begins filling, and that venting in the hub area is less adequate than venting in the rim and inboard flange, which leads to higher backpressure generated in the hub area.

The velocity plots (Figure 5.12) reveals that the flow in the sprue and rim is slow and quiescent and the velocities are generally less than $0.2 \mathrm{~m} / \mathrm{s}$. In contrast, since the spoke is effectively a downward channel, the flow rate increases to about $0.8 \mathrm{~m} / \mathrm{s}$ due to gravitational acceleration and the inlet pressure increase. Close-up views of the flow in the spoke (Figure 5.12 (b) and (c)) clearly show a backflow when the fluid hits the wall of the outboard flange, and recirculation generated at the junction of the spoke and the outboard flange just below the free surface. Recirculating flows are also observed at the hub, as illustrated in Figure 5.12 (d)), when two streams of fluid traveling in opposing directions, meet. The shallow downward stream flows underneath the deeper upward flowing stream causing eddies to form at the interface below the surface. This flow phenomenon is potentially harmful since the fluid from the surface intersection of the two streams is flowing downwards, necessarily leading to a fold-in of the free surface. This fold-in of free surface could possibly lead to the oxide cracks found in the wheel hub discussed in Section 1.2. 


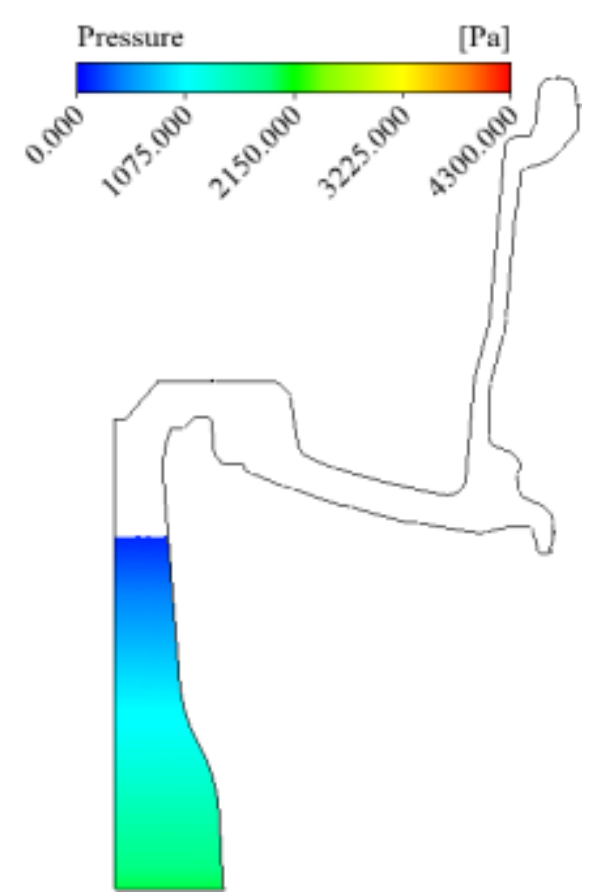

(a) $11 \mathrm{~s}$

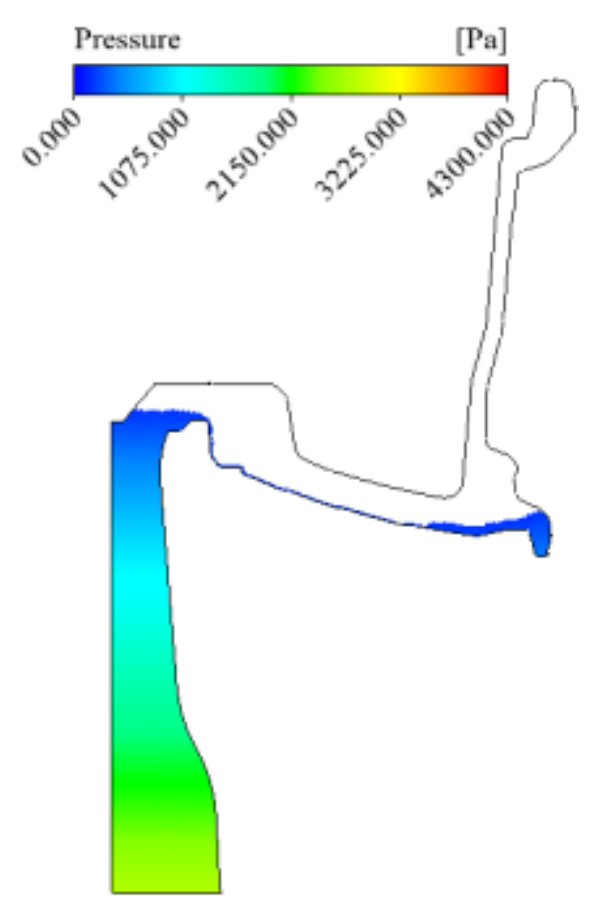

(b) $17 \mathrm{~s}$

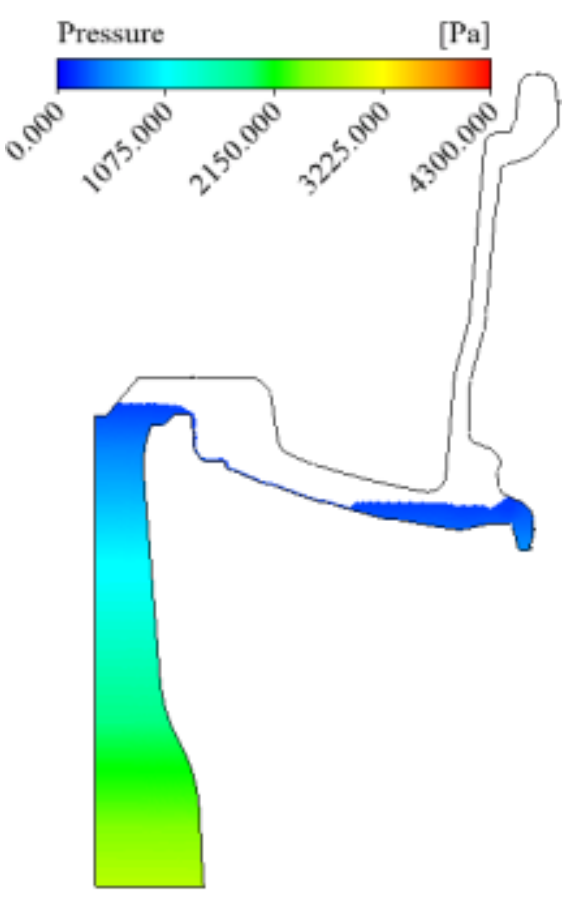

(c) $18 \mathrm{~s}$

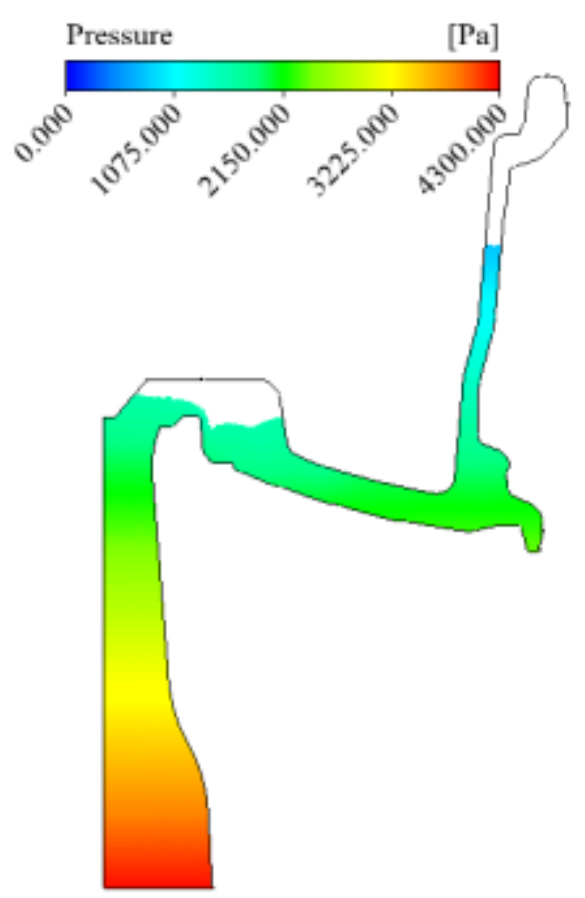

(d) $21 \mathrm{~s}$

Figure 5.11. Static pressure contour plots for water phase 


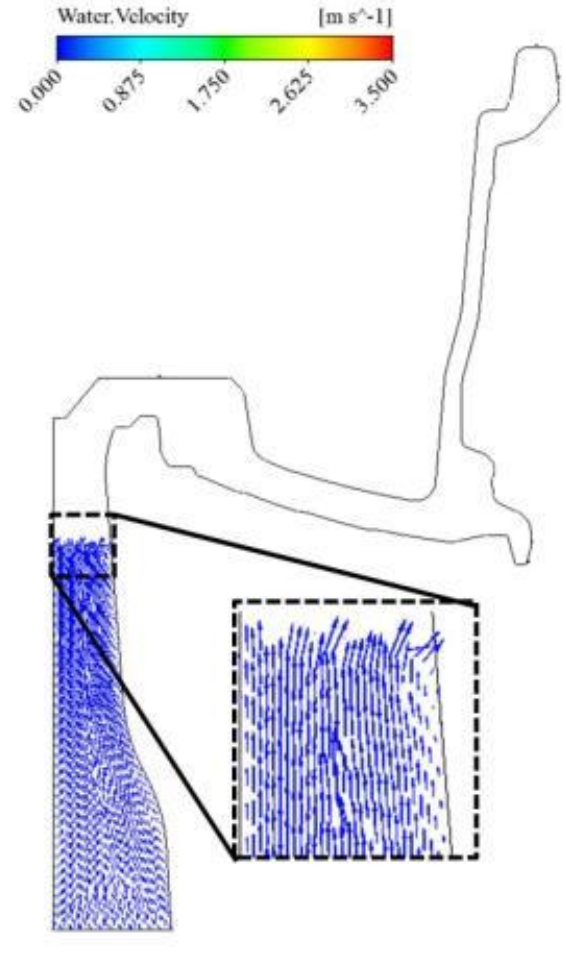

(a) $11 \mathrm{~s}$

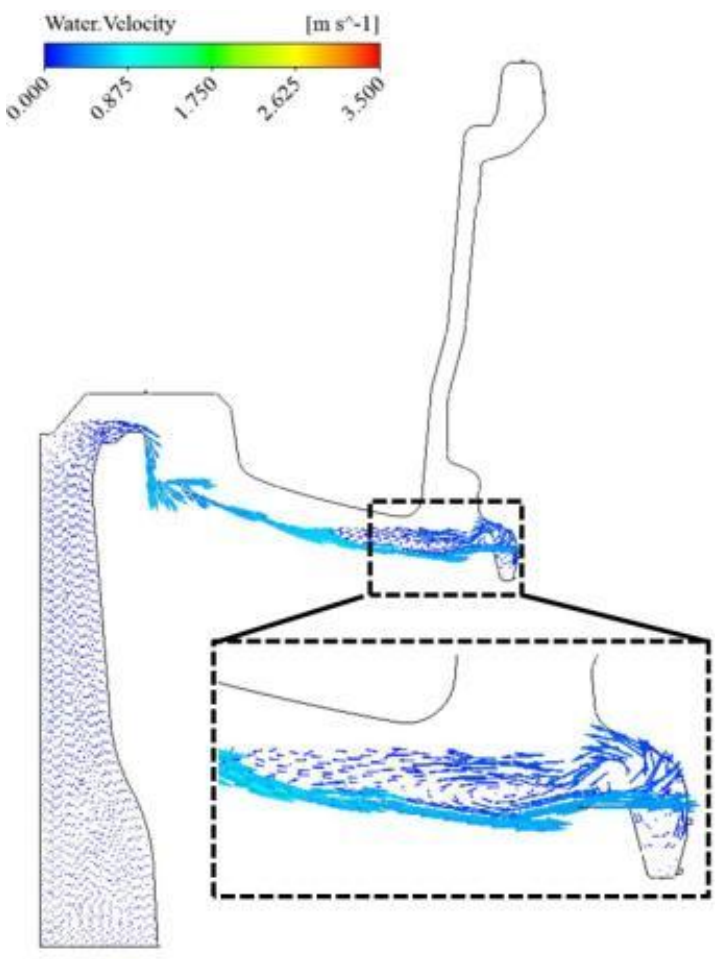

(c) $18 \mathrm{~s}$

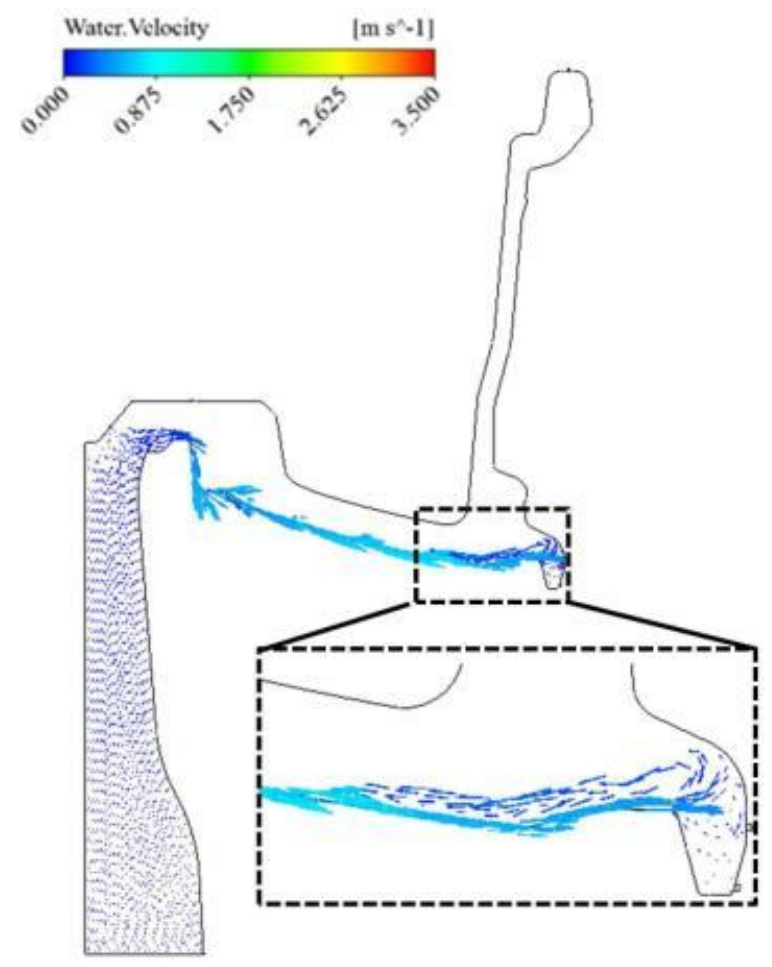

(b) $17 \mathrm{~s}$

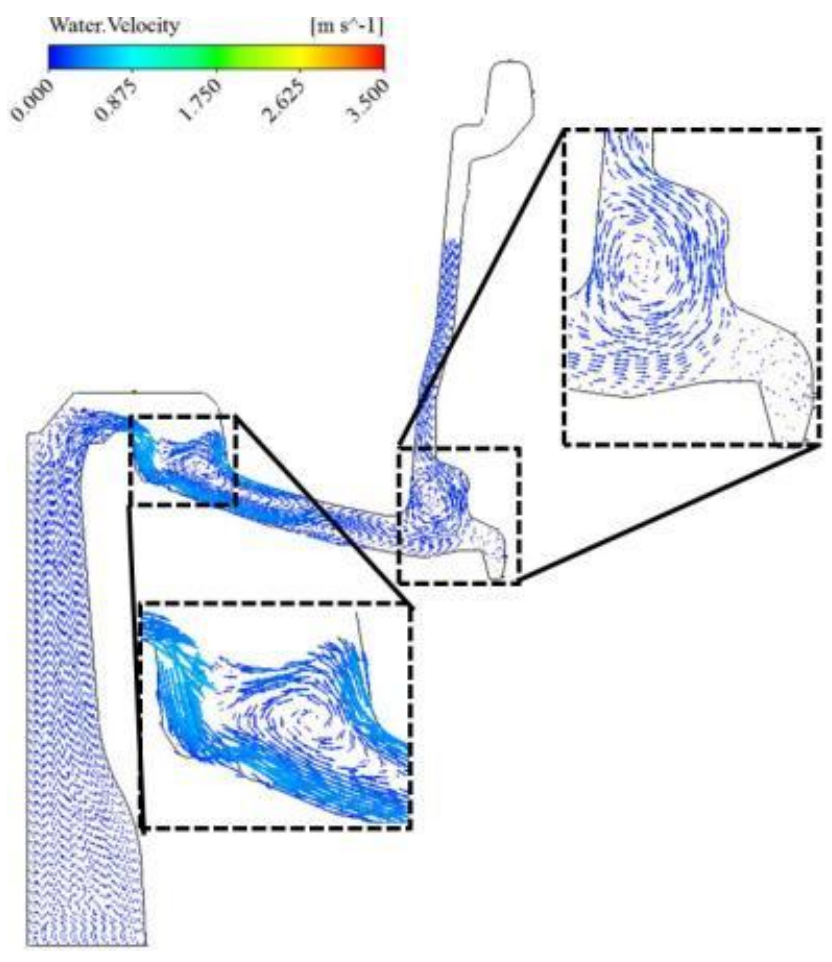

(d) $21 \mathrm{~s}$

Figure 5.12. Velocity magnitude and direction plots for water phase 


\section{Sensitivity Analysis}

A sensitivity analysis was performed with the filling model to elucidate the impact of some of the numerical and physical parameters used in the model on the die filling pattern. The factors investigated include the mesh size, the time step size, the type of flow, the surface tension, the adhesive force of the wall, and the vent resistance coefficients. The model developed to simulate the conditions of Test \#1 (refer to Section 4.2 Test Conditions) was used in this sensitivity study, and the numerical settings described in the Chapter 5 were employed as the baseline. Table 6.1 summarizes the factors that have been selected for the analysis.

Table 6.1. Summary of the factors investigated in the sensitivity study

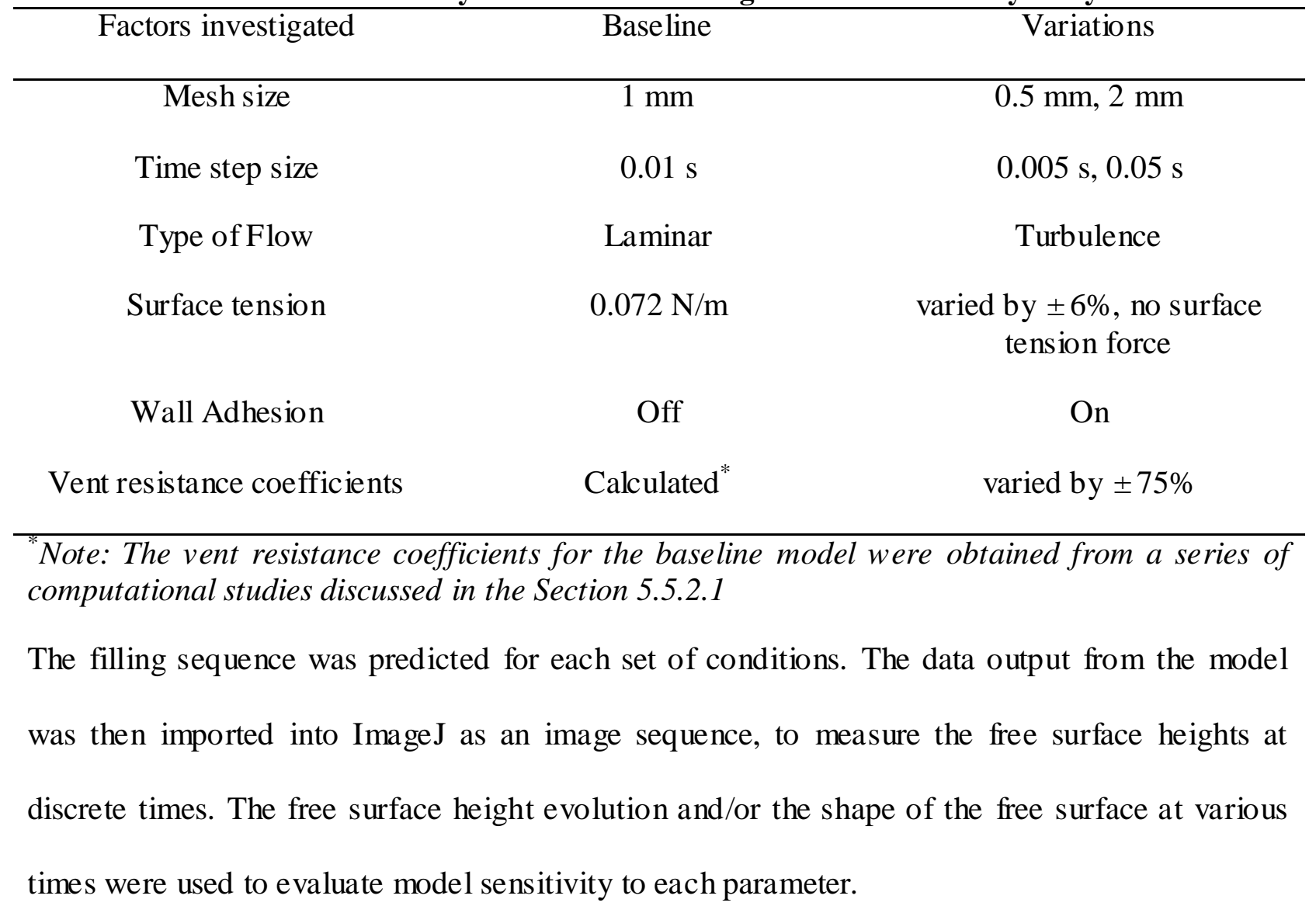




\subsection{Effect of Mesh Size}

The mesh size for the baseline model is $1 \mathrm{~mm}$, and it was increased and decreased by a factor of 2 during this sensitivity analysis. Table 6.2 summarizes the mesh information for these models. The time step in the model with $0.5 \mathrm{~mm}$ mesh size was changed from the baseline value of $0.01 \mathrm{~s}$ to $0.005 \mathrm{~s}$ to allow the model to converge. The time step was unchanged from the baseline model with the $2 \mathrm{~mm}$ mesh size. The computation times were $\sim 9.5$ hours, $\sim 25$ hours, and $\sim 252$ hours for the $2 \mathrm{~mm}, 1 \mathrm{~mm}$ (baseline), and $0.5 \mathrm{~mm}$ mesh size models, respectively.

\section{Table 6.2. Summary of mesh geometries}

\begin{tabular}{cccc}
\hline Model & Element Type & Nodes & Elements \\
\hline $0.5 \mathrm{~mm}$ mesh size & Hexahedra & 155458 & 76102 \\
Baseline, $1 \mathrm{~mm}$ & Hexahedra & 39916 & 19124 \\
$2 \mathrm{~mm}$ mesh size & Hexahedra & 10968 & 5032 \\
\hline
\end{tabular}

Figure 6.1 compares the variation of the free surface height with filling time for different meshes. The dashed line represents the predicted height evolution of the model with the $0.5 \mathrm{~mm}$ mesh size; the solid triangles represent the baseline model results obtained with $1 \mathrm{~mm}$ mesh size and the solid line is for the model with $2 \mathrm{~mm}$ mesh size. In each graph there are three sets of data plotted, which represent the height evolution in the sprue, in the spoke and in the rim. Filling within the sprue occurs between 0 and approximately $15 \mathrm{~s}$. After $15 \mathrm{~s}$, as the flow moves down the spoke, two free surfaces (with associated heights) form, which are plotted as the height of the fluid in the spoke and in the rim.

Turning to the results, no significant discrepancies were observed in the flow as the filling front moved through the sprue. The model with a $2 \mathrm{~mm}$ mesh size generally predicts a faster increase 
in height in the rim and spoke than the 1 and $0.5 \mathrm{~mm}$ meshes. However, apart from during filling of a portion of the rim, the deviation is relatively small. Under the assumption that the $0.5 \mathrm{~mm}$ solution produces the more accurate result, which is generally experienced in computational models of this type, a $1 \mathrm{~mm}$ mesh size is employed hereafter as it offers an approximately tenfold decrease in computational time without a significant compromise in accuracy.

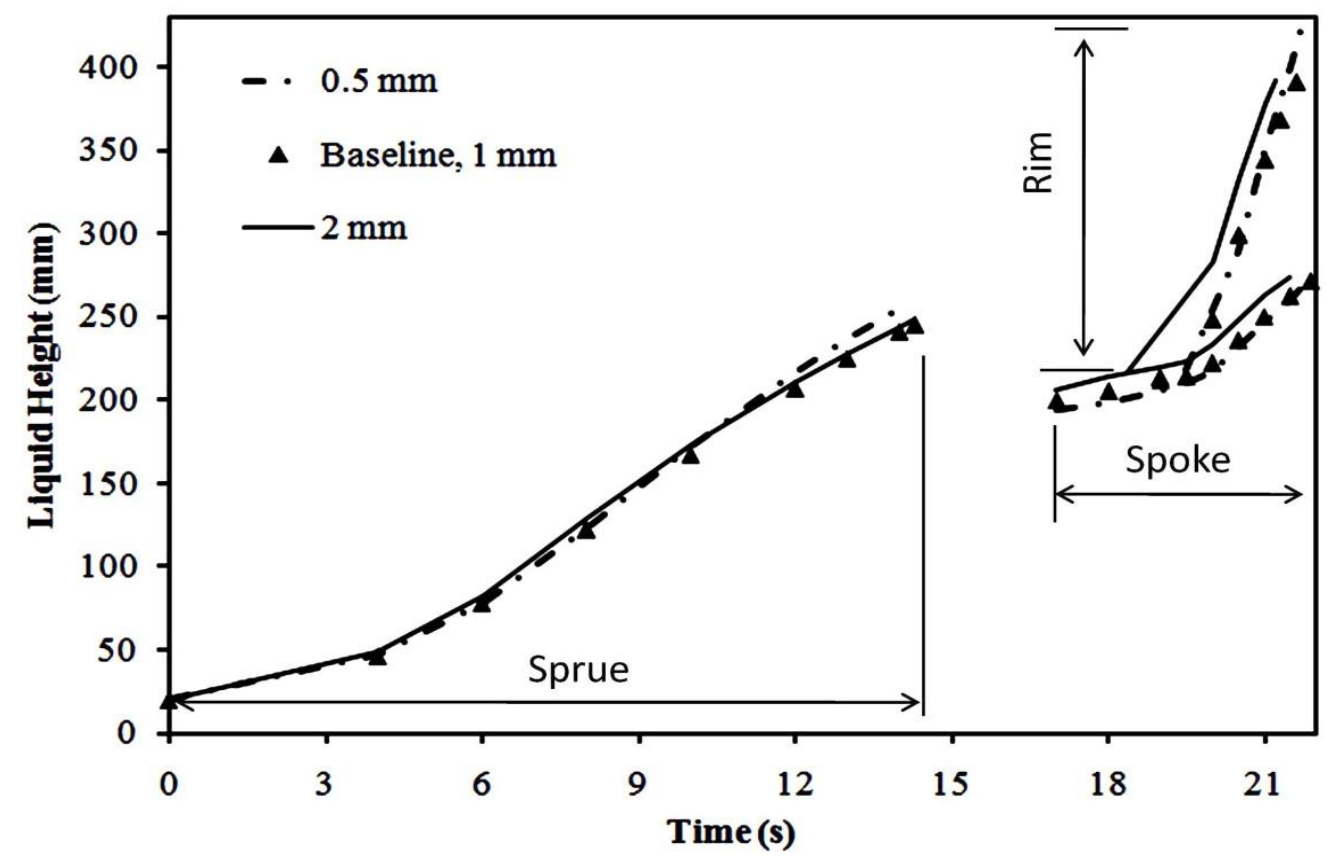

Figure 6.1. Comparison of free surface height versus fill time for the three models with mesh sizes of $0.5 \mathrm{~mm}, 1 \mathrm{~mm}$ and $2 \mathrm{~mm}$

\subsection{Effect of Time Step}

To examine the effect of time step size on the numerical results, three different time steps, $0.005 \mathrm{~s}, 0.01 \mathrm{~s}$, and $0.05 \mathrm{~s}$ were used and the evolution of free surface height compared in the sprue, the spoke and the rim. There are potentially two impacts of time-step size on the results one, pertaining to the ability of the computational model to converge and resolve transients in the flow field and the second, related to resolution of the transient pressure filling curve which is input to the model. 
The results of the simulations are compared in Figure 6.2. The curves for time step sizes of 0.005 and $0.01 \mathrm{~s}$ generally coincide with maximum differences observed to be approximately $4.3 \mathrm{~mm}$ in the sprue, $11 \mathrm{~mm}$ in the spoke and $30.5 \mathrm{~mm}$ in the rim, which are negligible (maximum 7\%) compared to the total height of wheel $(436.8 \mathrm{~mm})$. The curve for the time step size of $0.05 \mathrm{~s}$ shows a much different trend. The filing process is slowed in the sprue with the result that no fluid was present in the spoke and rim by the end of the pressure sequence specified in the models, which is not observed in practice. The results indicate that $0.05 \mathrm{~s}$ is too large a time step size for the filling model resulting in a significant error. The results for time steps of $0.005 \mathrm{~s}$ and $0.01 \mathrm{~s}$ show negligible difference, and support the use of a time step size of $0.01 \mathrm{~s}$ in the filling model for the sake of computational efficiency.

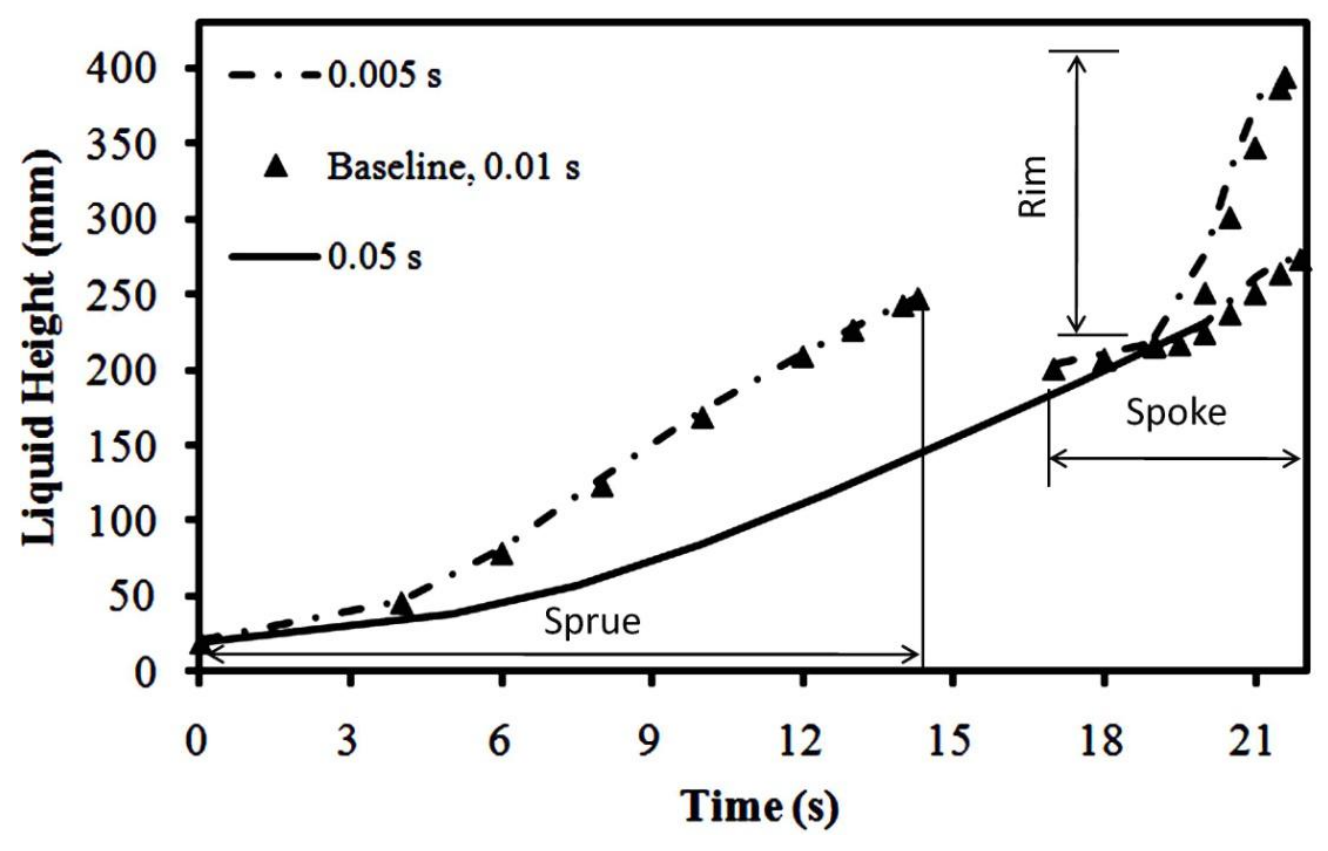

Figure 6.2. Comparison of free surface height versus fill time for the three models with time step sizes of $0.005 \mathrm{~s}, 0.01 \mathrm{~s}$ and $0.05 \mathrm{~s}$ 


\subsection{Effect of Flow Type}

The conventional way to assess the degree to which turbulence is present is the Reynolds number $(\operatorname{Re}=\rho v d / \mu)$. A value of $\operatorname{Re}$ above 2040 normally indicates the onset of turbulence in a pipe [90]. The average Re number in the water physical model, calculated by the average velocity obtained from the computational model is around 3800 for the water phase, which places the flow in the transition regime from laminar to turbulent. To assess the effect of including turbulence, the k-Epsilon turbulence model was used and the results, in the form of water volume fraction plots, were compared to those from the laminar model. The turbulence intensity was set to $5 \%$ for the whole fluid domain including inlet and outlet boundaries, which is an intermediate value within the range recommended [89].

Plots of the predicted water volume fraction at times of $11,17,18$, and $21 \mathrm{~s}$ are presented in Figure 6.3 and Figure 6.4 for the baseline model and the model that includes turbulence, respectively. The difference observed is that the depth of the stream of the returning wave in the spoke (Figure 6.3 (c) and Figure 6.4 (c)) was slightly larger and the free surface slightly smoother and flatter when the turbulence model was turned on. However, this distinction was not seen to affect the overall fill pattern, thus the effect of turbulence was deemed negligible. The computation time for the k-Epsilon turbulence model was $\sim 32 \mathrm{~h}$, compared to $\sim 25 \mathrm{~h}$ for the laminar model, or a $28 \%$ increase in the computation time. Due to the minimal impact of ignoring turbulence and the decreased computation time, the laminar model was used for the bulk of the analyses. 


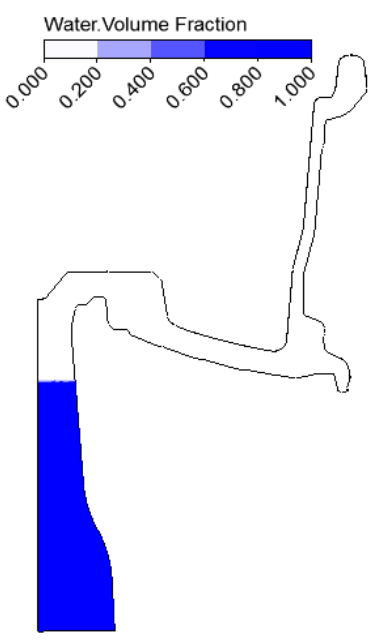

(a) $11 \mathrm{~s}$

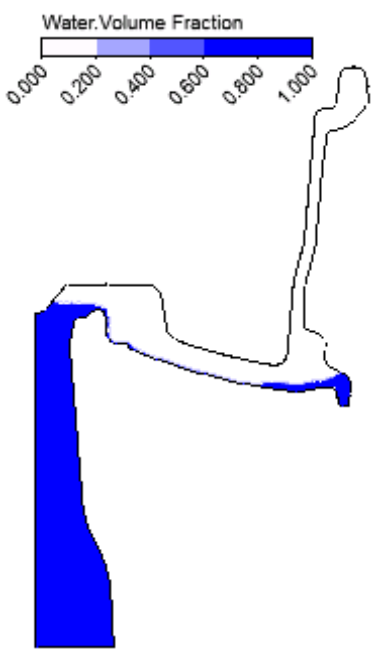

(b) $17 \mathrm{~s}$

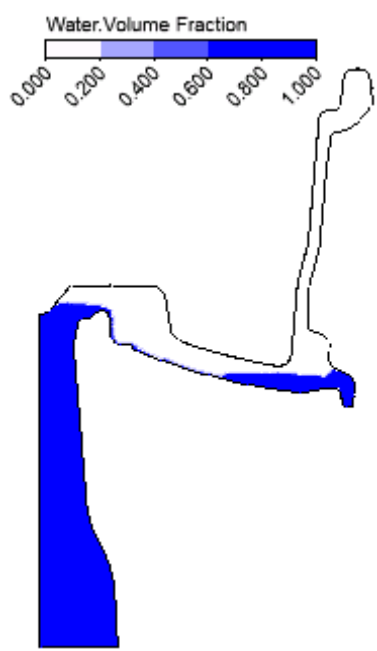

(c) $18 \mathrm{~s}$

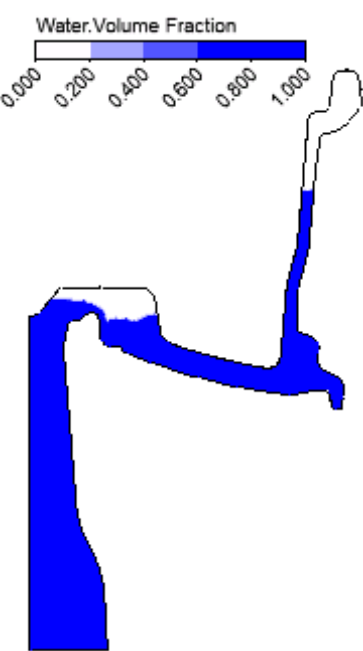

(d) $21 \mathrm{~s}$

Figure 6.3. Water volume fraction plots for the baseline model

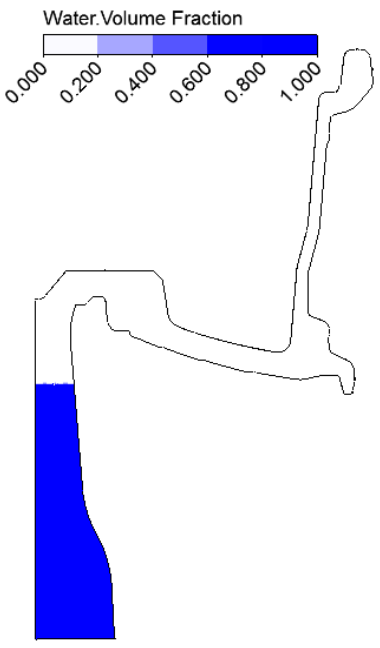

(a) $11 \mathrm{~s}$

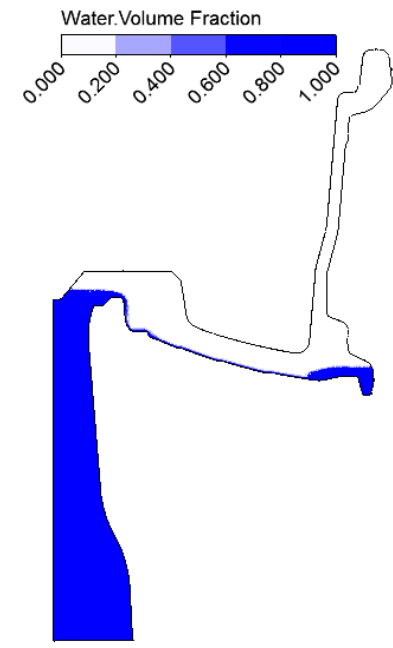

(b) $17 \mathrm{~s}$

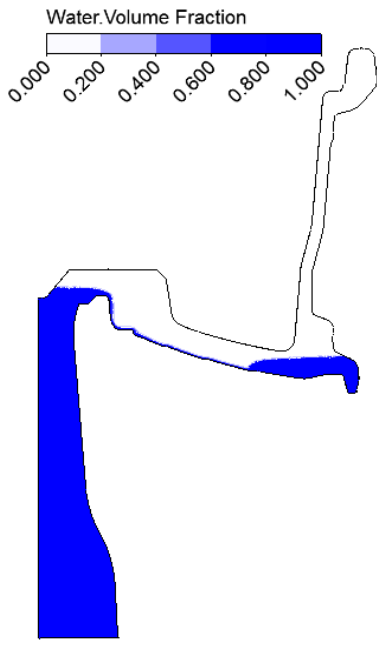

(c) $18 \mathrm{~s}$

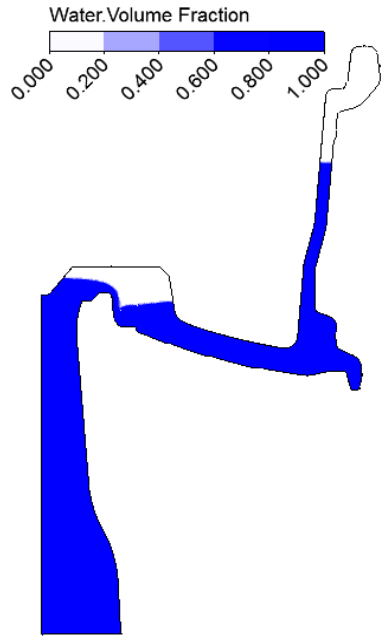

(d) $21 \mathrm{~s}$

Figure 6.4. Water volume fraction plots for the model that include turbulence

\subsection{Effect of Surface Tension}

The surface tension value $(0.072 \mathrm{~N} / \mathrm{m})$ for water and air at $25^{\circ} \mathrm{C}$ was obtained from literature. However, it was observed that the water temperature varied during the experiments. This temperature difference can lead to a difference in the surface tension and could potentially affect the flow pattern. The surface tension variation between $0{ }^{\circ} \mathrm{C}$ and $50{ }^{\circ} \mathrm{C}$ is approximately 
$12 \%$ [89], thus the surface tension was varied by $\pm 6 \%$ to investigate the effect of surface tension during filling expecting a nominal water temperature of $25^{\circ} \mathrm{C}$.

The free surface levelevolution for the models in which the surface tension was varied is plotted in Figure 6.5. Increasing or decreasing the surface tension by $6 \%$ was not seen to change the transient liquid level evolution. At the same time, the free surface shape was not seen to vary. The result suggests that a $6 \%$ change in surface tension is unlikely to affect the filling pattern in the wheel.

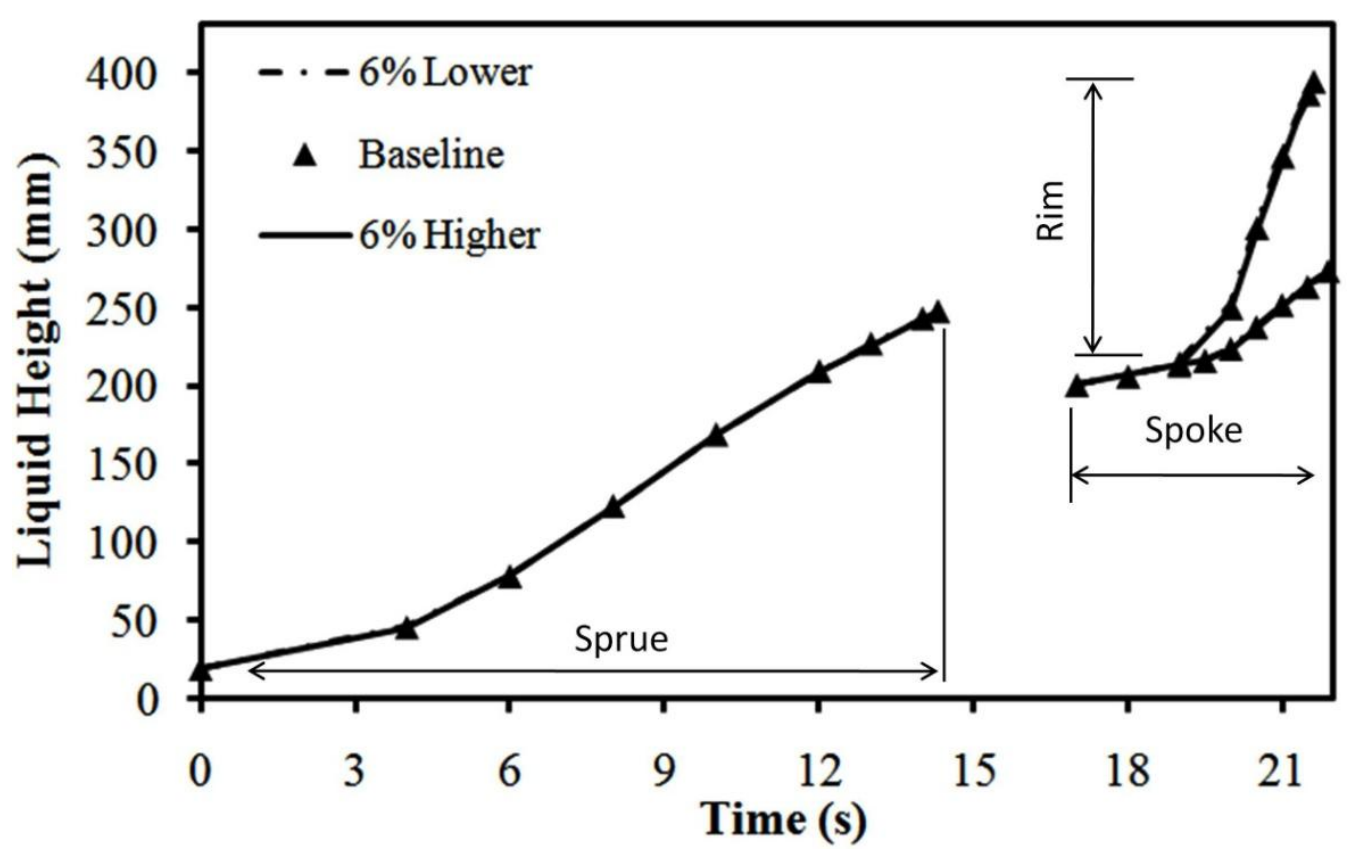

Figure 6.5. Comparison of free surface he ight for $6 \%$ lower surface te nsion, baseline surface tension and $6 \%$ higher surface tension

To assess the importance of including surface tension effects, another model was run without the surface tension effects. For numerical convergence, the time step size was changed to $0.1 \mathrm{~s}$ and the maximum iterations per time step were set to 20 . The results, in the form of a series of water volume plots, are presented in Figure 6.6. By comparison with Figure 6.3 it can be seen that when surface tension is removed as the fluid falls over the ornament area into the spoke, it 
separates from the vertical wall as it moves into and down the spoke whereas in Figure 6.3, this is observed not to be the case.

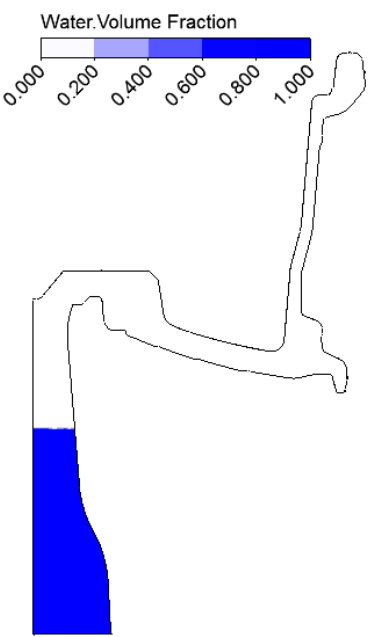

(a) $11 \mathrm{~s}$

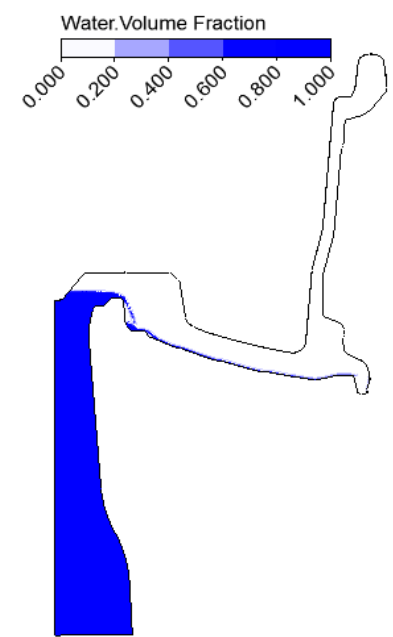

(b) $17 \mathrm{~s}$

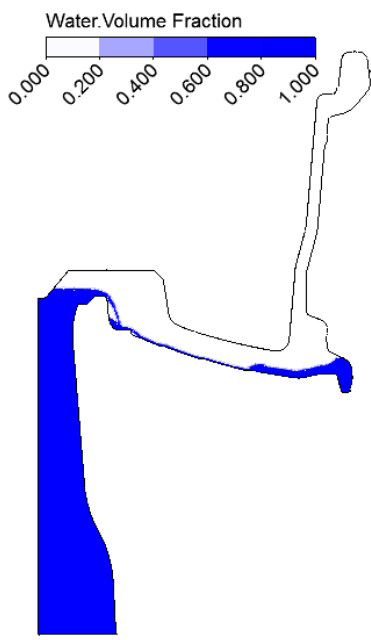

(c) $18 \mathrm{~s}$

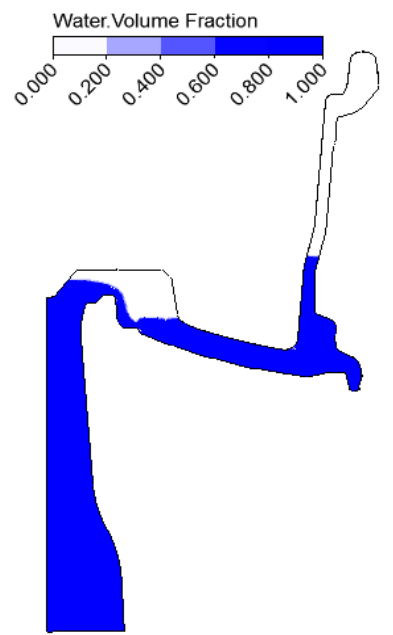

(d) $21 \mathrm{~s}$

Figure 6.6. Water volume fraction plots for the model with surface tension force inactive

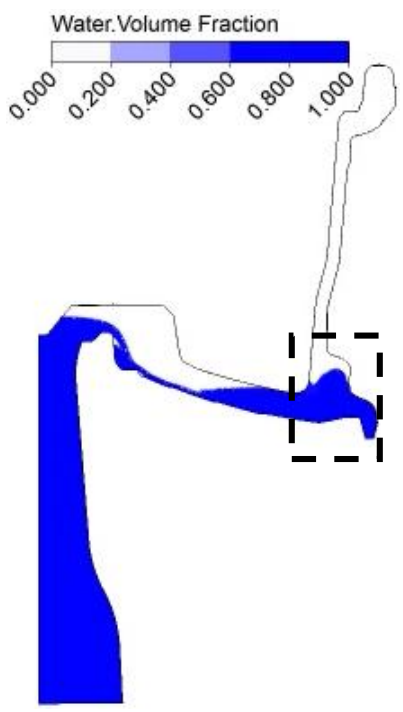

Figure 6.7. Water volume fraction plot for the model with surface tension force inactive, showing unreas onable free surface shape at the junction of the outboard flange and the rim at $20 \mathrm{~s}$

\subsection{Effect of Wall Adhesion}

When a liquid touches a solid, the liquid maybe attracted (wetting condition) or repelled (nonwetting condition). The degree to which the liquid is attracted depends on a balance of the 
surface tension forces and is defined as the contact angle. An angle larger than $90^{\circ}$ indicates a non-wetting condition and an angle less than $90^{\circ}$ indicates a wetting condition. This phenomenon, known as wall adhesion, gives rise to effects such as the meniscus and the capillary rise in small tubes under static conditions [89]. Wall adhesion is expected to be much less influential in a dynamic situation such as during die filling, due to dominance of the momentum term associated with the moving liquid. However, since wettability affects how the liquid interacts with walls, it could affect the way the free surface behaves during filling.

Based on this assumption, a model that includes the wall adhesion force was set up. A contact angle of $72^{\circ}$ was applied to the mold wall [91]. Figure 6.8 compares the free surfaces near the Hub vent in the physical water model (Figure 6.8 (a)), the baseline model, which does not include wall adhesion, (Figure 6.8 (b)) and the model that includes adhesion (Figure 6.8 (c)). It is clear that the wall adhesion model helps to correctly characterize the free surface shape in the region examined.

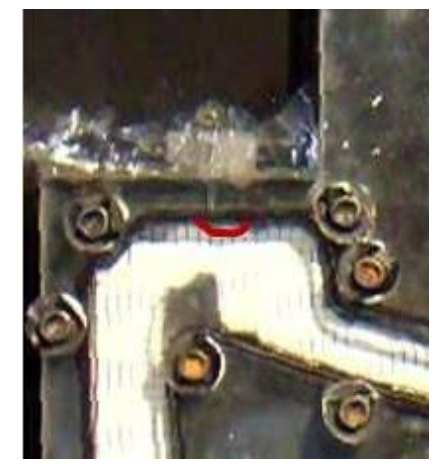

(a) Expe riment

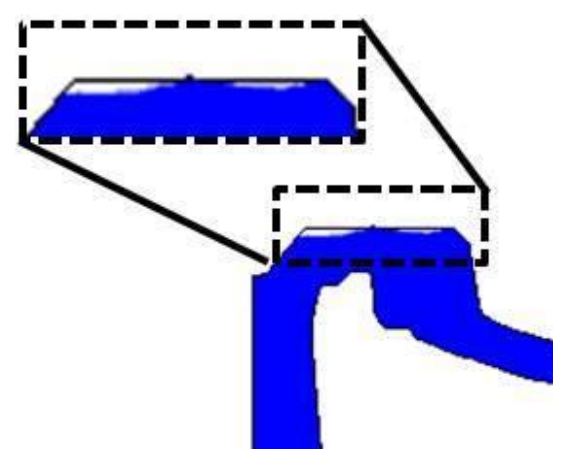

(b) Baseline model

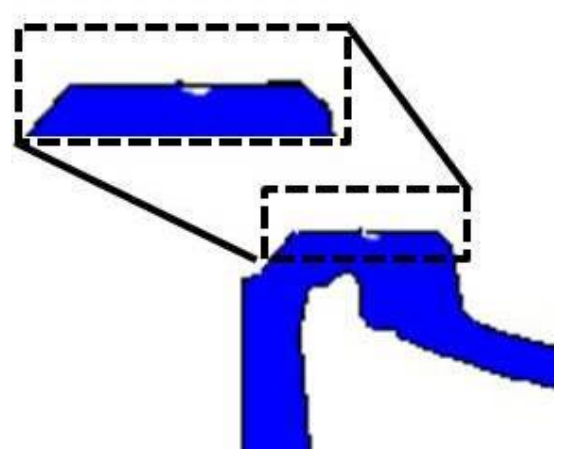

(c) Wall adhesion model

Figure 6.8. Comparison of the effect of wall adhesion on the free surface shape below the hub vent (images have been cropped)

A comparison of the predicted flows in other areas however, indicated some issues. When comparing the water volume fraction plots of the model considering wall adhesion (Figure 6.9) to the baseline model (Figure 6.3), the filling was considerably slower. Closer examination of the 
flow in the spoke shows discontinuities in the liquid stream (Figure 6.10 (a)) and free surface shapes (Figure 6.10 (b)) not observed in the flow in the spoke of the physical model. Thus on balance, to ensure an optimum accuracy of the model, wall adhesion was not included in other analyses.

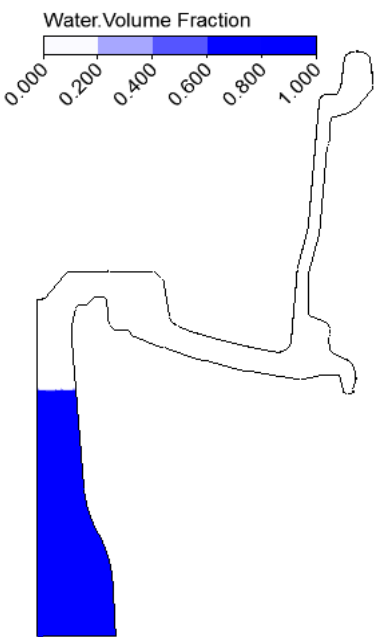

(a) $11 \mathrm{~s}$

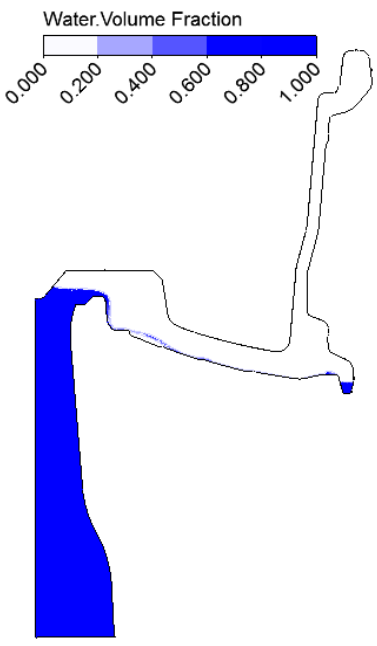

(b) $17 \mathrm{~s}$

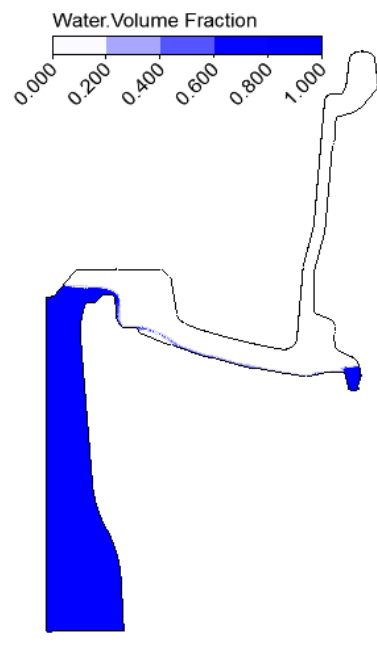

(c) $18 \mathrm{~s}$

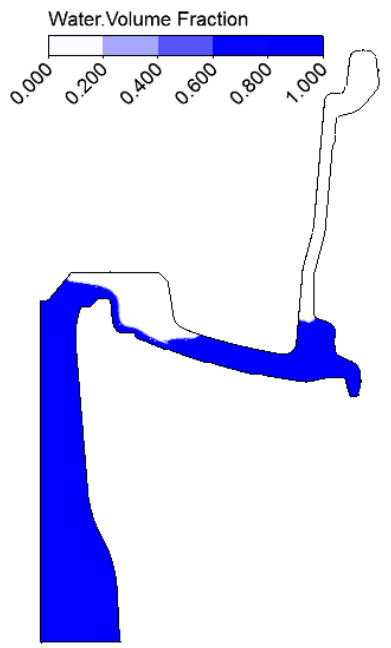

(d) $21 \mathrm{~s}$

Figure 6.9. Water volume fraction plots for the model that include wall wettability effect

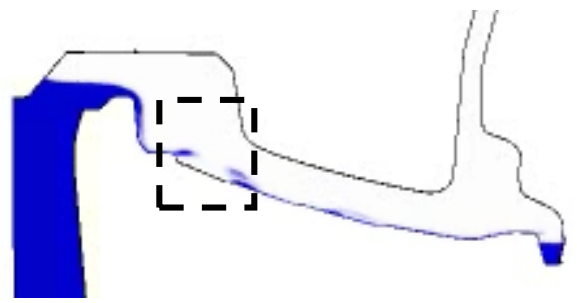

(a) Discontinuity in the liquid

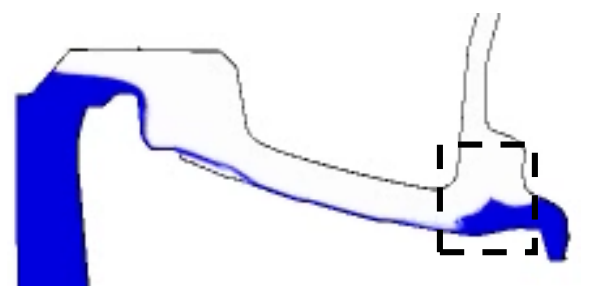

(b) An example of unreasonable free surface shape

Figure 6.10. Water volume fraction plot for the model with wall we ttability model, showing (a) discontinuity in the liquid, and (b) an example of unreasonable flow behavior (images are cropped)

\subsection{Effect of Vent Resistance Coefficients}

The vent resistance coefficients were determined through computational modeling of the estimated experimental vent geometry (refer to Chapter 5), however, vents at different locations have different dimensions which will lead to different resistances to flow. Thus, the sensitivity of 
model results to vent resistance was assessed by either increasing or decreasing the vent resistance by $75 \%$. The resistance coefficients for the three models are shown in Table 6.3. A summary of the flow's sensitivity to the venting condition can be found in Figure 6.11.

Table 6.3. Vent resistance coefficients for baseline, resistance increased $75 \%$, and resistance decreased $75 \%$

\begin{tabular}{ccc}
\hline Models & $\mathrm{K}_{\text {perm }}\left(\mathrm{m}^{2}\right)$ & $\mathrm{K}_{\text {loss }}\left(\mathrm{m}^{-1}\right)$ \\
\hline Baseline & $1.34 \times 10^{-10}$ & 1168051 \\
Resistance increased $75 \%$ & $7.63 \times 10^{-11}$ & $2.04 \times 10^{6}$ \\
Resistance decreased $75 \%$ & $5.34 \times 10^{-10}$ & 292013 \\
\hline
\end{tabular}

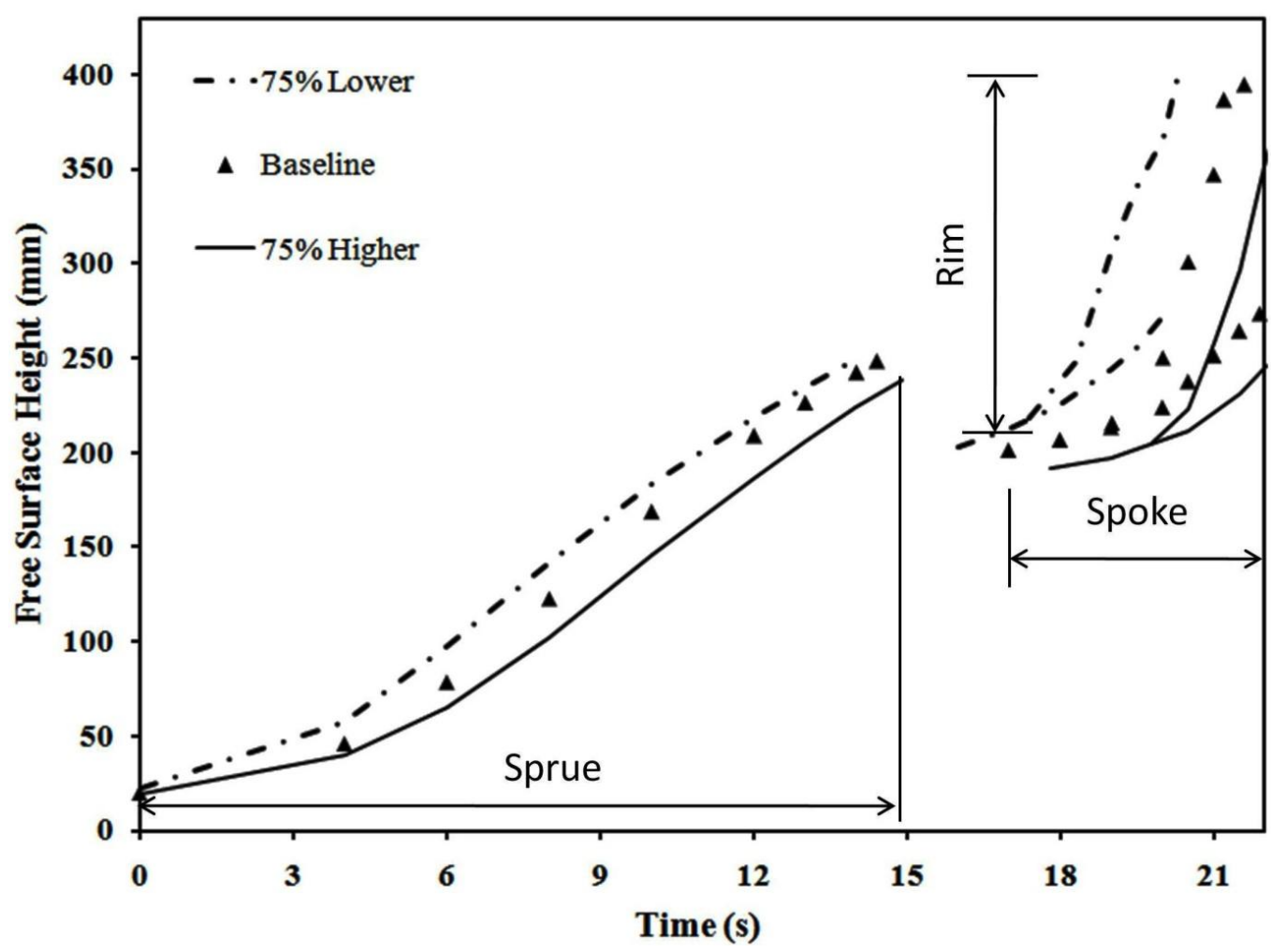

Figure 6.11. Comparis on of free surface height versus fill time for the three models with different vent resistance coefficients

Figure 6.11 shows that the fluid height in the sprue, the hub and the rim are affected by vent resistance. For example, range run in the model resulted in a maximum height difference of 
around $50 \mathrm{~mm}$ in the spoke and over $100 \mathrm{~mm}$ in the rim at given time. Die filling times were predicted to be $21.8 \mathrm{~s}, 22.5 \mathrm{~s}$, and $23.4 \mathrm{~s}$ for the resistance decreased, the baseline, and resistance increase, respectively. The data clearly shows that a decreased in the momentum loss at the vent results in a decreased backpressure, thus the liquid advances faster and less time is required to fill the die. 


\section{RESULTS AND DISCUSSION}

The over arching goal of this research program is to investigate the flow behavior in a Low-Pressure Die Cast (LPDC) aluminum alloy automotive wheel during die filling. The rational for this work is that the liquid free surface behavior can influence several defects that remain a challenge to control. There are at least two major factors that can alter the flow behavior during the LPDC die filling process. One is the geometry of the die cavity, and the other is the velocity of the liquid metal entering the die cavity. The latter being determined by a combination of pressure applied at the inlet to the cavity and the venting conditions.

In this chapter, the effects of the venting conditions and the inlet pressure will be discussed in the context of the water analogue model. While the die geometry is certainly an influential factor, it is typically dictated by mechanical and aesthetic considerations. Designing and altering die geometry is beyond the scope of this work. In contrast, altering venting conditions or modifying the inlet pressure profile is much easier to implement in an industrial context and test in both a physical model (in a lab setting) and in a computational model. The ultimate goal is to set the stage for understanding the generation of oxide-film related defects and to also hopefully gain some valuable insights that can be applied quickly to improve the industrial process.

\subsection{Study of Venting Conditions}

As discussed in Section 2.3.1 Die Filling, air is restricted from leaving the die cavity during filling, causing pressure to build in the cavity, and affects the fill pattern. The increased pressure that develops is referred to as the backpressure. Given the same flow rate, the magnitude of the backpressure is dependent on the venting ability of the die which is based on three elements: the venting capacity of each vent, the number of vents and the location of the vents. 


\subsubsection{Venting Capacity - Assessment and Preliminary Computational model}

\section{Validation}

The sensitivity of the flow to the vent resistance coefficients input to the computational model has already been presented in Section 6.6) and clearly showed that the computational model predictions are sensitive to the venting conditions - e.g. over the range of vent resistances examined the backpressure was sufficient to impact on filling. In this section, the results will be further analyzed by comparing the free surface height evolution predicted by the computational model with the experimental results. In addition, a series of curves have been added that show the evolution of free surface height in the sprue, spoke and rim as calculated according to Pascal's Law $(h=P / \rho g)$ using the experimental pressure readings.

The experimental results obtained from the water model and Pascal's law results are shown plotted in Figure 7.1 together with the computational model results. As can be seen in Figure 7.1, it is clear that the experimental data (like the computational model data) show a lower free surface height at a given time than the results generated applying Pascal's Law (note: that the large discrepancy at the transition from the sprue to the spoke/rim is due to the drop in height that occurs in the spoke that cannot be accounted for using Pascal's relationship). Since the flow velocity during die filling is quite low, especially in the sprue region, the time offset between the curve predicted by Pascal's Law and the curves for the computational and experimental results is likely due to inadequate venting and the development of backpressure. Therefore, in the current water model design, venting has the potential to impact on the flow conditions as predicted earlier in the numerical analysis.

As previously described, three computational models with different vent resistance coefficients ($75 \%$, baseline and $+75 \%$ ) were run to evaluate the effect of venting capacity. A comparison 
between the numerically derived free surface heights and the experimental data shows that the case with the vent resistance coefficients $75 \%$ lower than the baseline has the best fit. This indicates that the loss coefficient values originally estimated from detailed computational analysis of the vent geometry were overestimated.

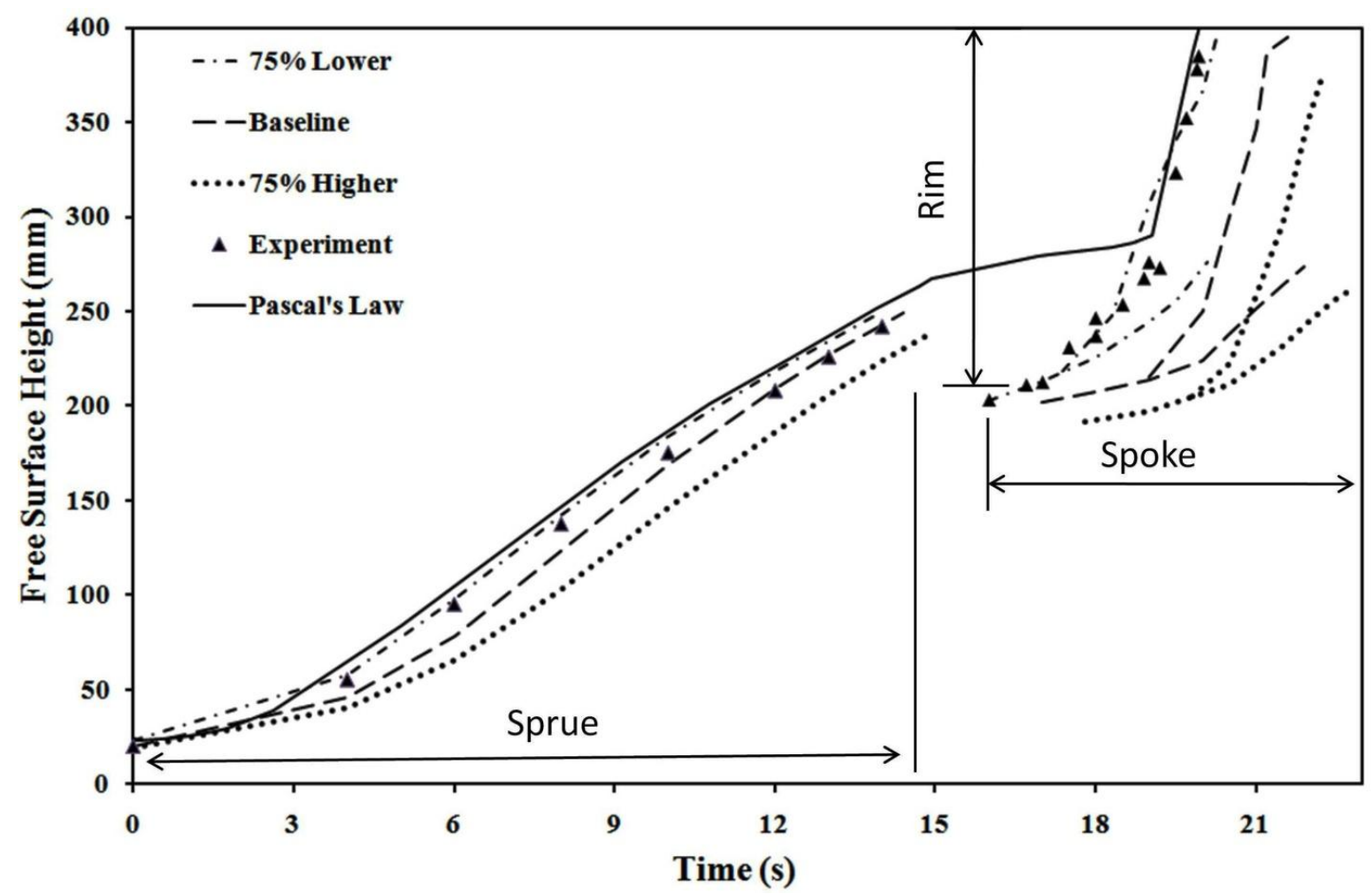

Figure 7.1. Comparison of the free surface height between computational models with diffe rent vent capacities and the physical model. Results of Test \#1 shown.

Upon further review, each vent is comprised of a $\$ 2 \mathrm{~mm}$ hole bored from the outside of the die, which is connected to the die cavity via a $\phi 1 \mathrm{~mm}$ hole, as shown in Figure 5.5 in Chapter 5. In the computational model the length of each bore hole was estimated to be $20 \mathrm{~mm}$, giving a total vent length of $40 \mathrm{~mm}$. In the experimental die, the lengths of the vents at different locations are different as shown in Table 7.1. It is clear that the original estimate of $20 \mathrm{~mm}$ for each bore length was excessive in all but one case. In particular it is worth noting that $\phi 1 \mathrm{~mm}$ holes, which will result in the largest momentum losses, were all over estimated. For example, the $\phi 1 \mathrm{~mm}$ 
bore hole in the hub is just $6.4 \mathrm{~mm}$, or about $1 / 4$ of original estimate of $20 \mathrm{~mm}$. Fluid mechanics principles show that the loss coefficient is proportional to the length [92]. Thus, it is not surprising that given the shorter actual lengths, the $-75 \%$ case results in a better fit to the experimental data as shown in Figure 7.1.

Table 7.1. Vent dimensions in the experimental die

\begin{tabular}{ccc}
\hline Vent Location & $\phi 2 \mathrm{~mm}$ Section & $\phi 1 \mathrm{~mm}$ Section \\
\hline Hub Vent & $17.6 \mathrm{~mm}$ & $6.4 \mathrm{~mm}$ \\
Riser Vent & $11.4 \mathrm{~mm}$ & $8.6 \mathrm{~mm}$ \\
Inboard Flange Vent & $14.7 \mathrm{~mm}$ & $17.8 \mathrm{~mm}$ \\
Outboard Flange Vent & $28 \mathrm{~mm}$ & $18.5 \mathrm{~mm}$ \\
\hline
\end{tabular}

\subsubsection{Critical Phenomena during Filling}

Figure 7.2 shows images taken from the experimental filling video that represent some examples of free surface entrainment phenomena observed during die filling. In one example, fluid can be seen to accelerate as it falls down the spoke channel towards the outboard flange. The high velocity causes the entrainment of an air bubble of over $10 \mathrm{~mm}$ in diameter (shown in Insert 1). In another example, when the outboard flange is filled, a wave of fluid is reflected off of the wall travelling back up the spoke. This phenomenon is commonly referred to as a returning wave. As the returning wave travels up the spoke toward the hub, it meets with the stream of liquid from the die inlet flowing down the spoke. These two opposing flows generate eddies at their intersection which results in surface turbulence. If this occurs in a casting process, the surface oxide film can become entrained resulting in a defect in the final product. 
Inserts 2 and 3 in Figure 7.2 show bubbles with diameters of $1 \sim 3 \mathrm{~mm}$ entrained below the free surface of the returning wave. In some other cases, bubbles at $\sim 0.5 \mathrm{~mm}$ were observed. In the casting process, the entrained bubbles may rise and escape through the free surface leaving a collapsed tube of oxide, which is referred to as a bubble trail - see Section 2.2.2.5. Alternatively, depending on the flow, some of the bubbles may remain trapped in the liquid to form large pores upon solidification.

Finally, when the liquid free surface in the spoke reaches the hub, there is a noticeable fold-in of the free surface in this region. This is shown in Inserts 4 and 5 of Figure 7.2. The curvature of the interface of the two opposing streams identifies a potential spot for free surface entrainment. Surface films entrained in these areas are very likely to contribute to the oxide cracks observed in the hub area (refer to Figure 1.1 in Chapter 1), and may lead to the rejection of the wheel. An air bubble of $\sim 2.5 \mathrm{~mm}$ in diameter was observed at this location in the water model. It should be noted that the bubble seen on the edge in Insert 5 is not from air entrainment but was caused by air which was trapped in the gaps between die plates.

Turning to the computational model results, shown in Figure 7.1, the case with vent resistance lowered by $75 \%$ results in the best reproduction of free surface entrainment related phenomena observed in the water model experiments. For example, a large bubble was predicted to be generated and entrained in the outboard flange (the first image of Figure 7.3) and a clear fold-in of the free surface was predicted in both the spoke (the third image of Figure 7.3) and the hub (the fourth image of Figure 7.3). However, the small bubbles (diameters between $\sim 0.5$ and $\sim 3 \mathrm{~mm}$ ) that were identified in the experimental filling video below the free surface of the returning wave in the spoke, and at the junction of the spoke and hub, were not predicted by the model. The inability of the model to predict these bubbles may be due to that the element size used in the model $(1 \mathrm{~mm})$ is too large to resolve the small bubbles with diameters close to $1 \mathrm{~mm}$. 

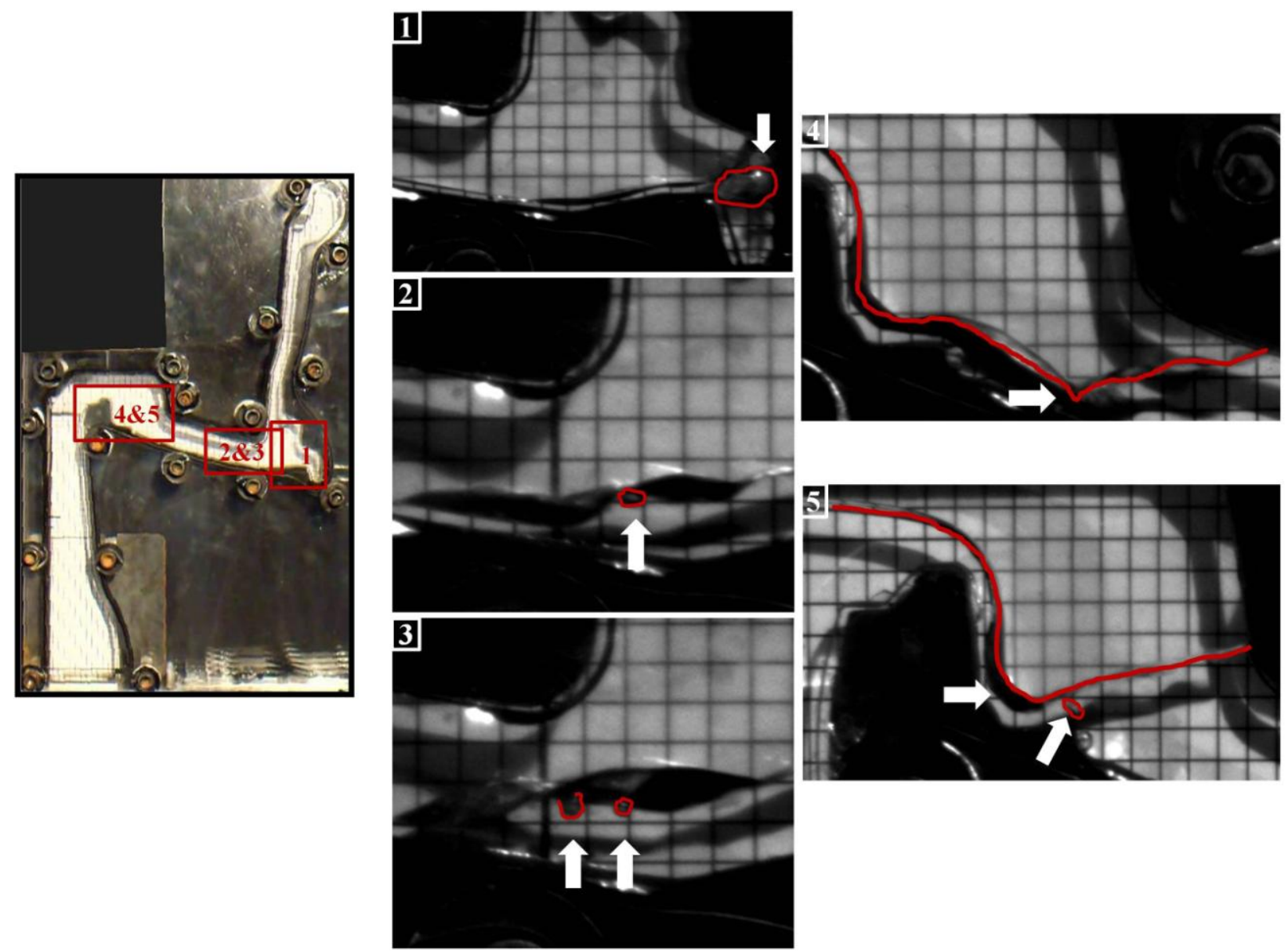

Figure 7.2. Images showing defect entraining filling phenomena seen during the die filling process:

1) bubble in outboard flange, 2) \& 3) bubbles below the free surface, 4) fold-in of the free surface, and 5 ) potential fold-in of the free surface and bubble entrained below it. The shape of the free surface and bubbles are marked by lines. 

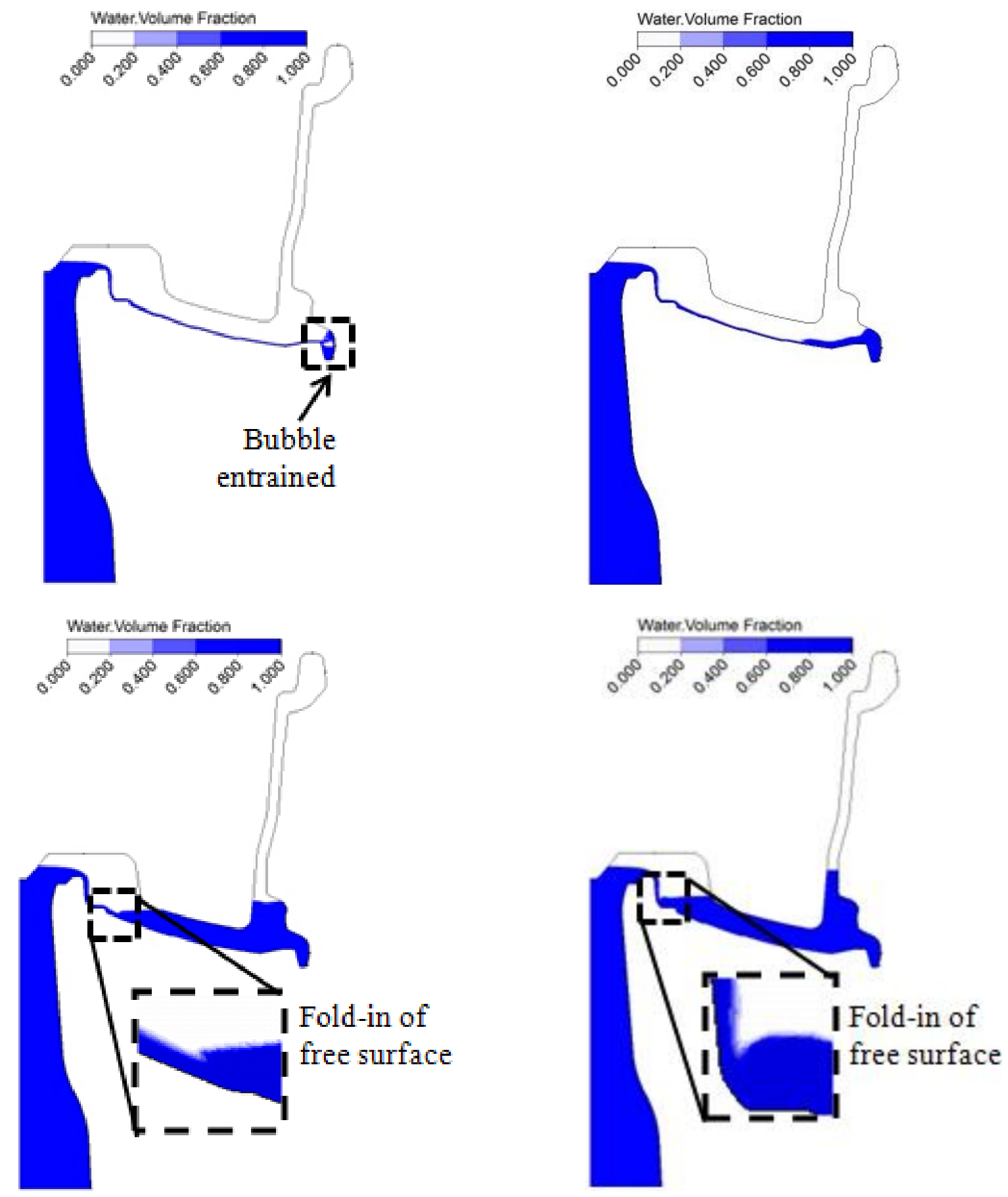

Figure 7.3. Free surface plots for the case with vent resis tance coefficients lowered by $75 \%$

It should be noted that there are no air bubble predicted in the baseline and increased vent resistance models. The free surface flow appears to be more quiescent in these models with higher vent resistance (higher backpressure). This implies that larger backpressure slows down the fluid flow and can potentially reduce free surface turbulence. 


\subsubsection{The Number and Locations of Vents - Final Validation}

Five tests with the same predefined pressure curve, but different venting conditions have been carried out using the water model in order to quantitatively evaluate the effect of venting on the fill pattern. As described in Table 4.3 of Chapter 4, Section 4.2, different venting conditions refer to different opening combinations of vents. And in the tests run, the individual vents were either fully closed or fully open in the tests.

To assess the results, the water model mold cavity was divided into discrete domains, or regions, as shown in Figure 7.4. To be defined as fully filled the volume fraction of water in the discrete domain has to be 1 , neglecting the presence of small gas bubbles. The time to fill was calculated based on the time relative to when the free surface passes the line at the bottom of the sprue marked by a line with notation 0 .

Figure 7.5 shows the time to fill the discrete domains within the cavity for the five different test conditions. Note that there are columns (bars) missing at some of the locations due to the fact that these domains did not fill under certain venting conditions. It can be seen that when the hub and riser vents are open (\#1 and \#3), regardless of the condition of the other vents, the die can be fully filled, and the time to fill all regions is quite similar. 


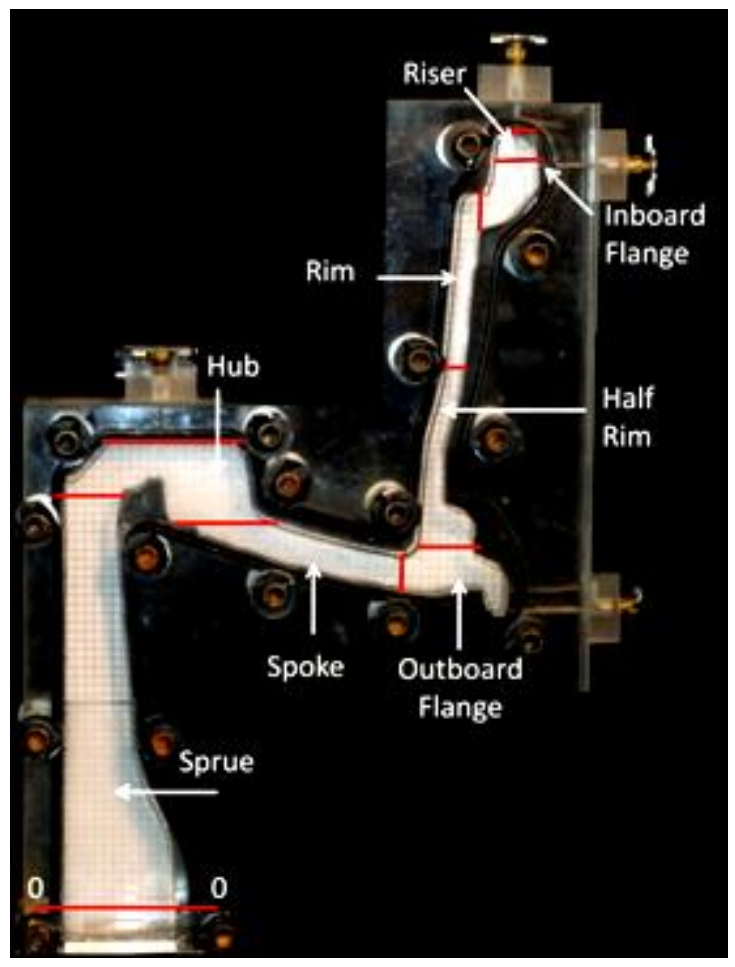

Figure 7.4. Definition of discre te domains within the wheel profile

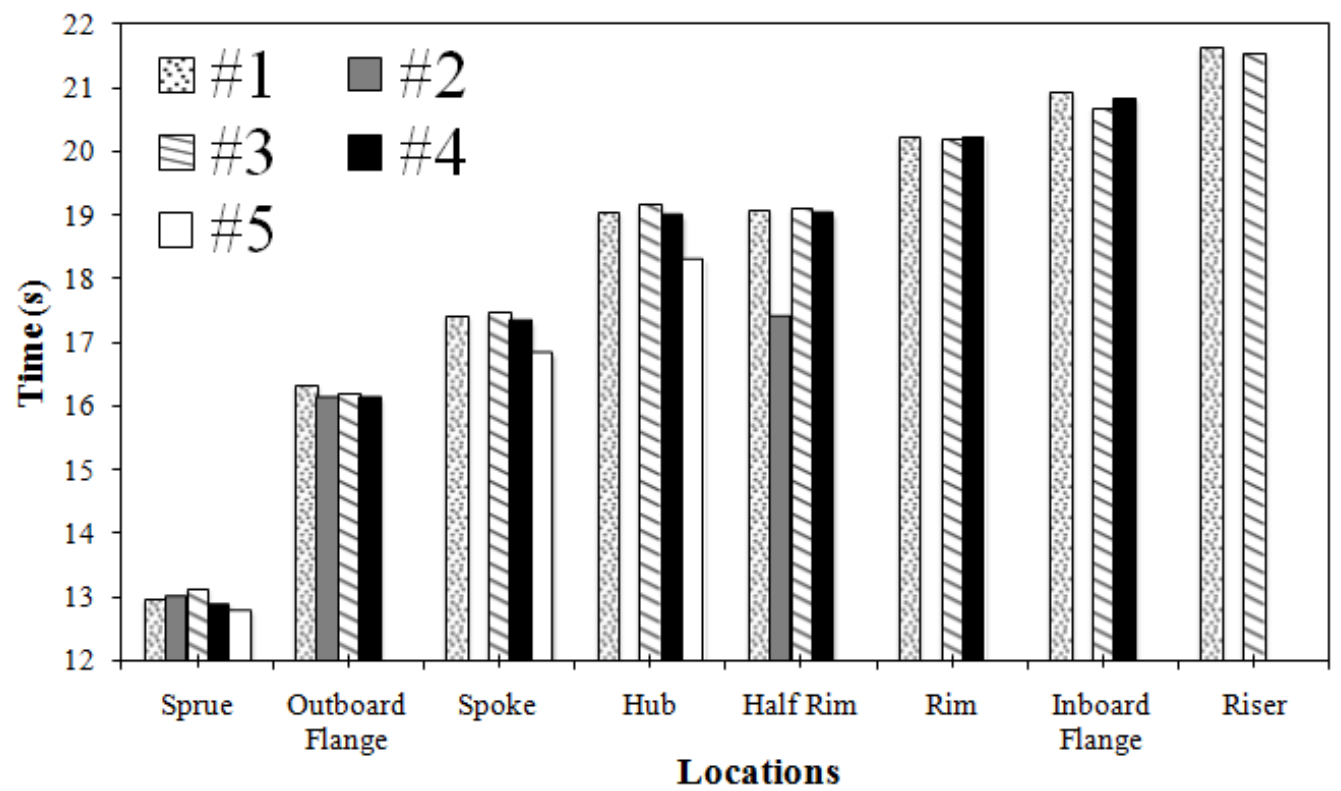

Figure 7.5. Time taken to fully fill discre te locations of the water model

For Tests \#1, \#3 and \#4, no significant time variations were observed for most locations except the riser. It is assumed that the automatic inlet control system has counteracted the effects of the vents, by maintaining the same static pressure in the different tests as it followed the same 
predefined pressure curve. It is anticipated that without automatic control, that is opening the electronic valve manually, the time variation would be much more appreciable. With regard to Test \#2, it takes significantly less time to fill half of the rim than the other conditions, but it then fails to fill the inboard flange, hub, and spoke locations within the defined pressure cycle. A check of the recorded pressure values shows that this phenomenon was largely an effect of the automatic control as well. In the lower half rim, the rate of pressure increase (height increase) due to the fact that the hub is not being filled, is larger than the changing rate in the predefined pressure curve. This results in the automatic control system closing the electronic valve as the upper part of the rim is being filled.

Figure 7.6 to Figure 7.10 show the fill pattern comparison between the experiment and model results for the five different venting combinations. Inspection of results indicates that the free surface shapes and surface turbulence severity correlate well between the physical and computational models. However, the fill rate calculated by the computational model is slightly smaller than that in the physical model. This difference is more pronounced in the spoke and hub regions than in the sprue and rim regions.

Table 6.3 summarizes the vent resistance coefficients used in the five models. In all but one case, the vent resistance coefficients used in this series of models are $75 \%$ lower than the baseline calculated values, as they have been proven to give a better description of the actual resistance of the vents in the experimental die (refer to Section 7.1.1). However, for Test \#5, where only the hub vent is open, the loss coefficients had to be further lowered to $90 \%$ of the baseline values to provide the fit shown in Figure 7.10.

These results clearly show that venting is a very important parameter in die design, however, the effects of venting are not thoroughly understood and no work in the literature exists to give 
quantitative effects of venting parameters (location, size and resistance). More research is required in this area.
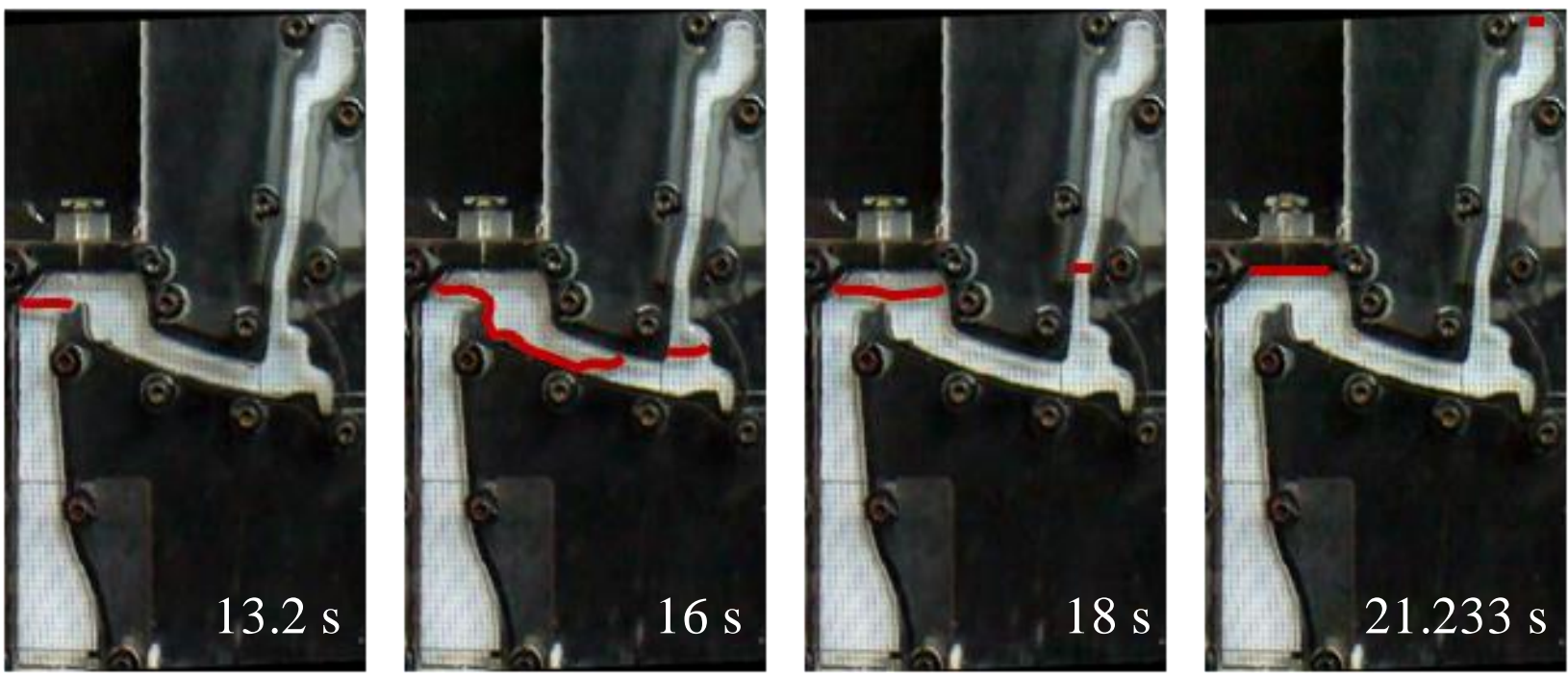

(a) Physical model
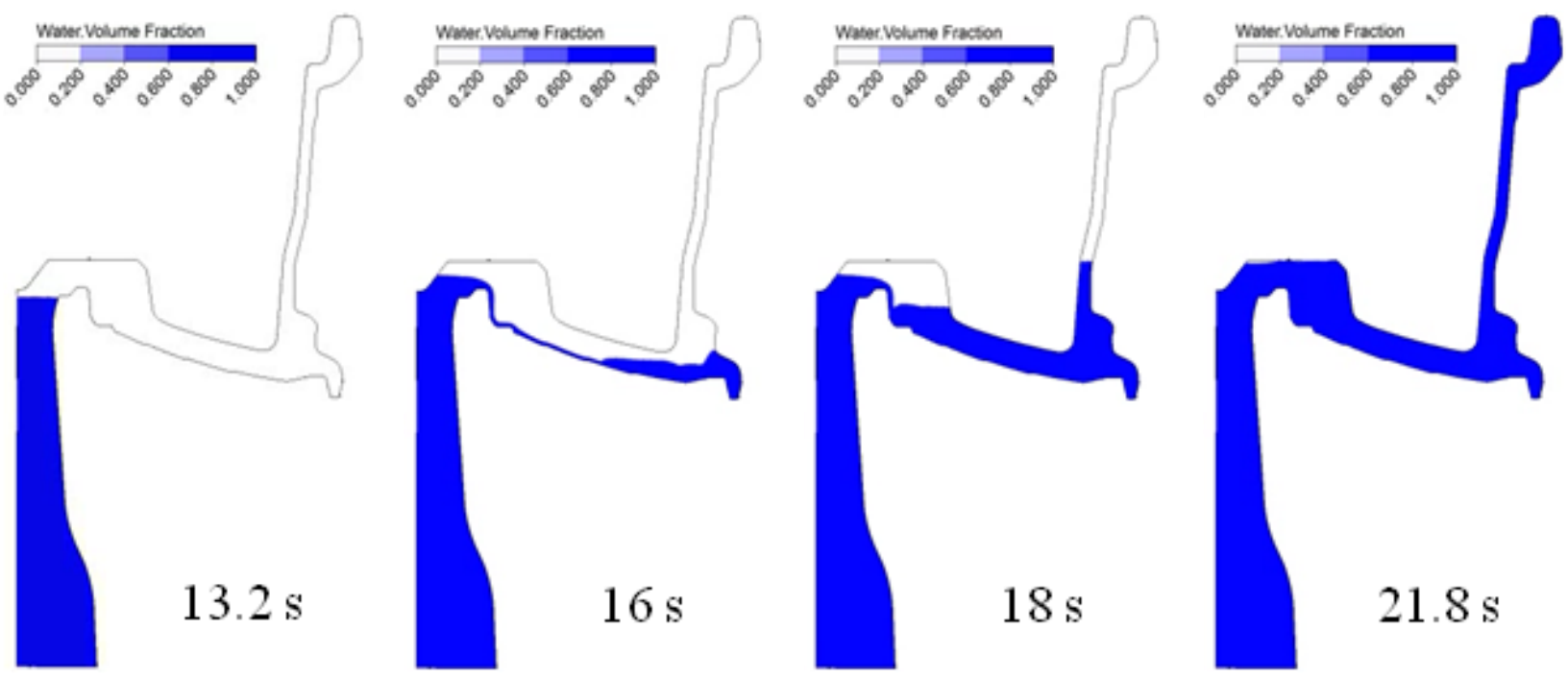

(b) Computational model

Figure 7.6. Comparison of the fill pattern between experimental result and the computational result for Test \#1 

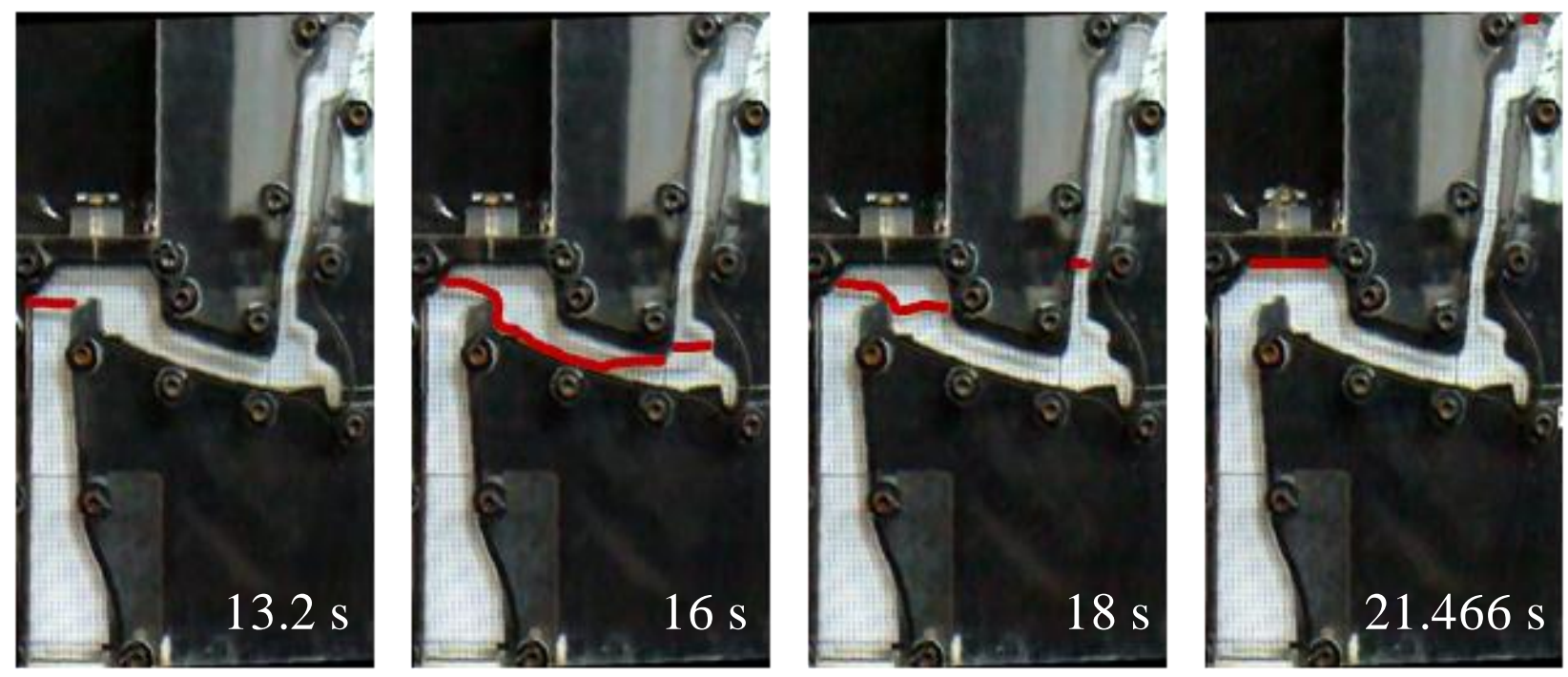

(a) Physical model
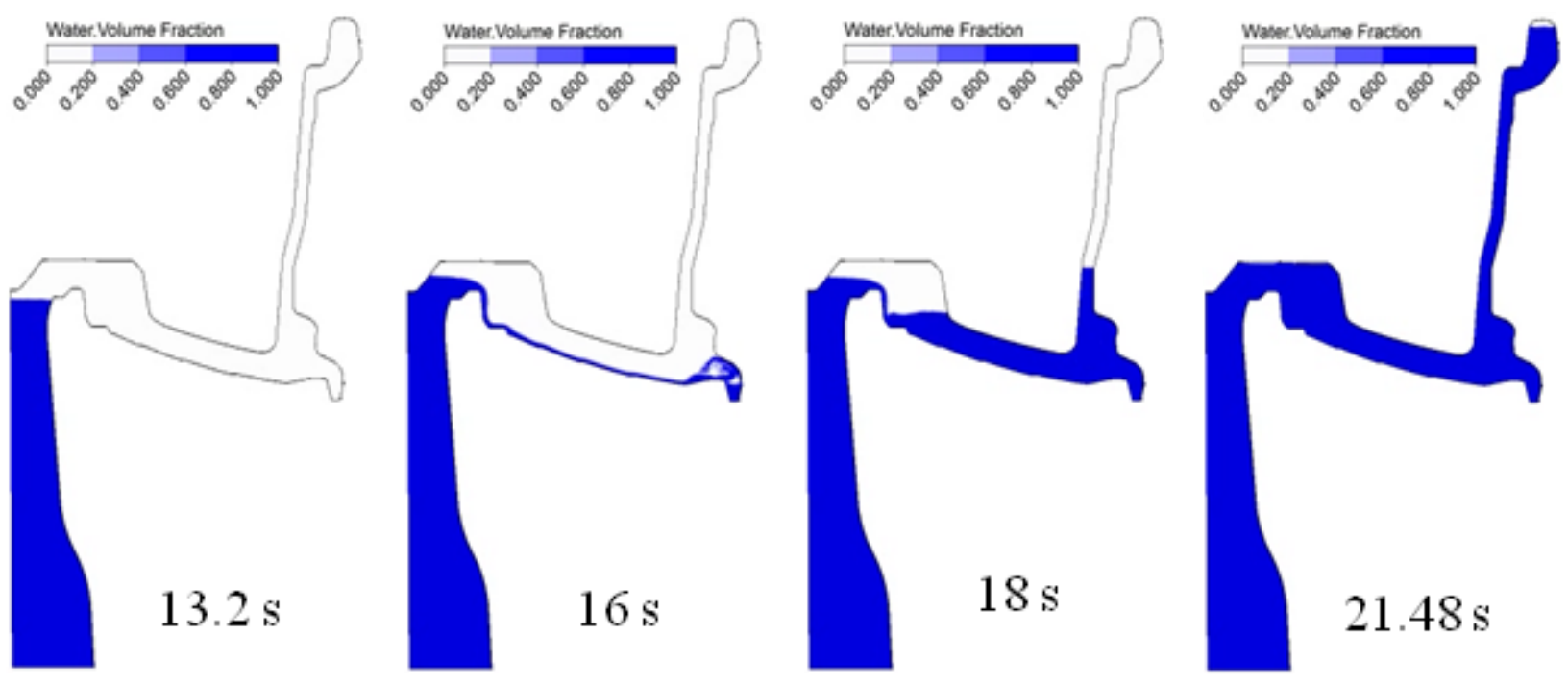

(b) Computational model

Figure 7.7. Comparison of the fill pattern between experimental result and the computational result for Test \#2 

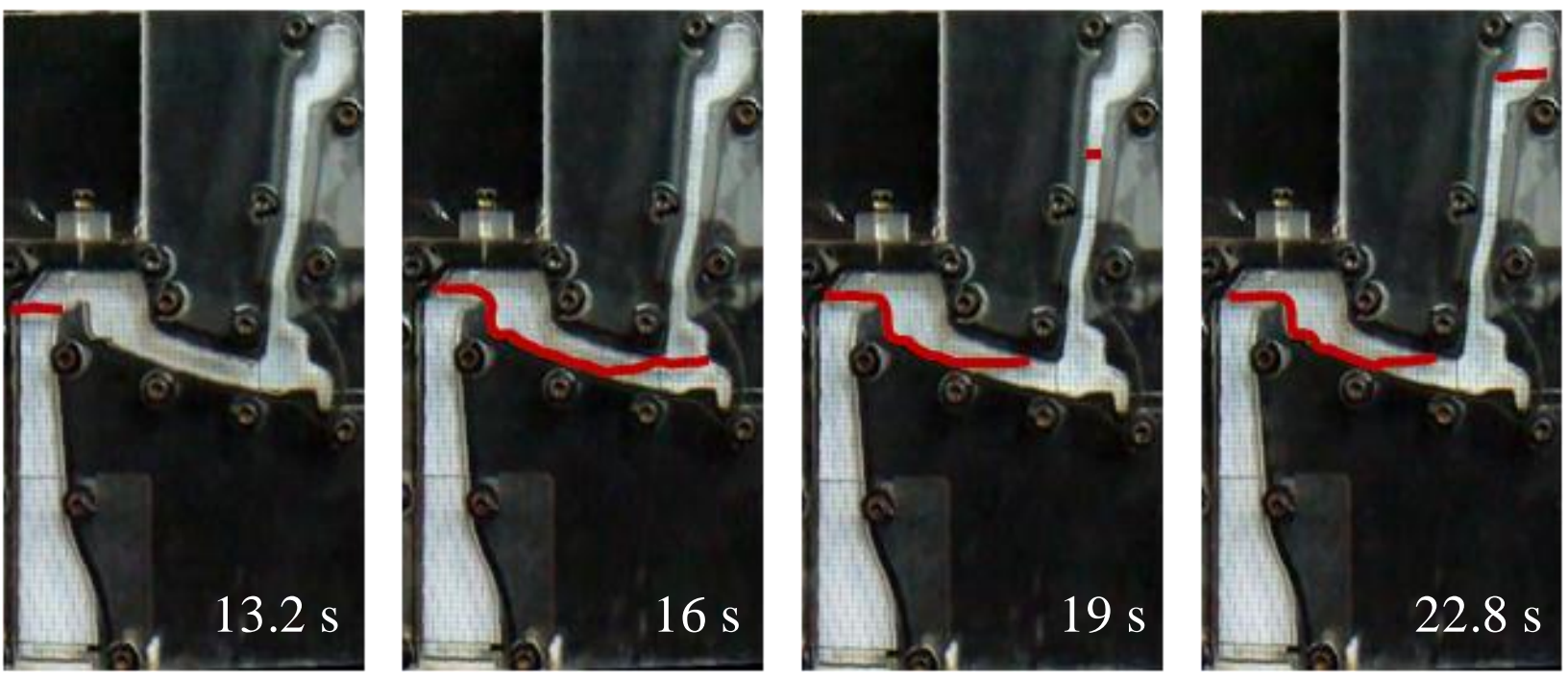

(a) Physical model
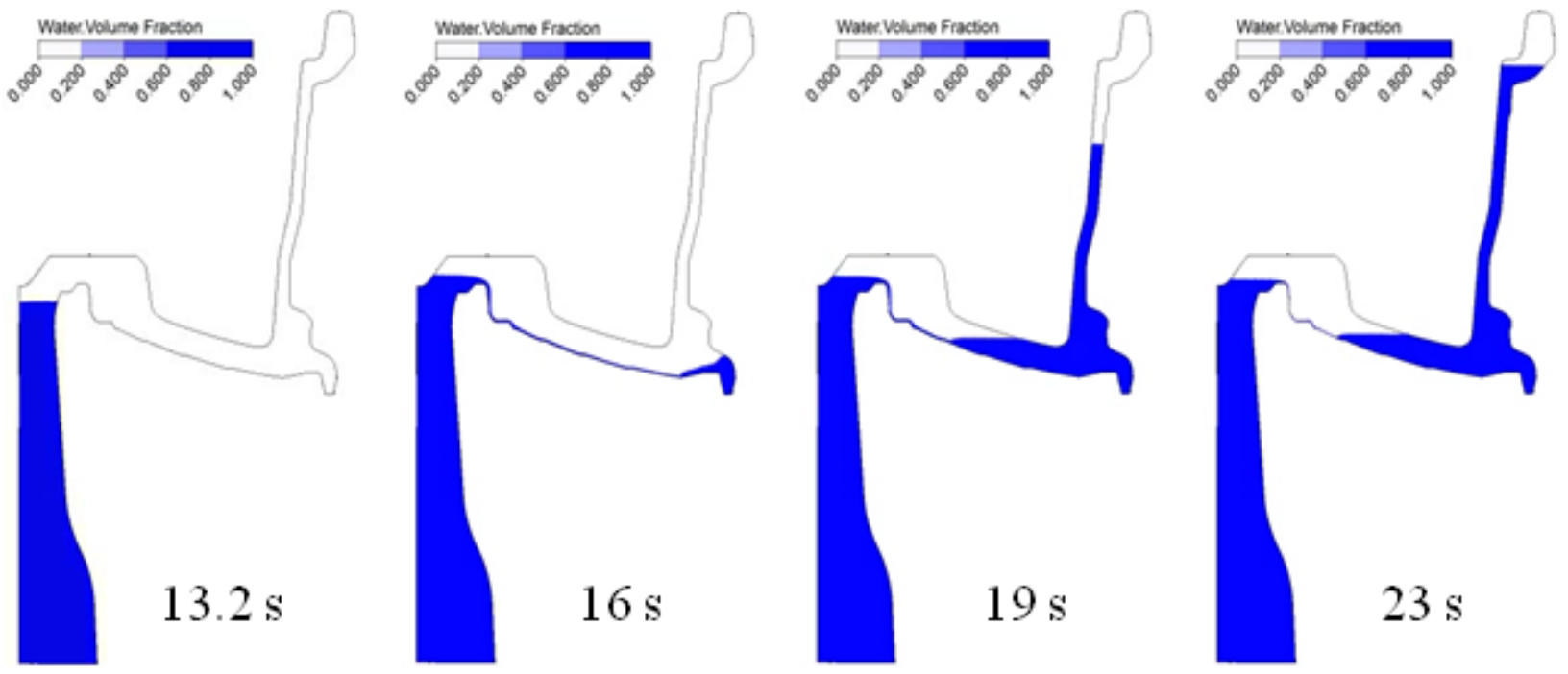

(b) Computational model

Figure 7.8. Comparison of the fill pattern between experimental result and the computational result for Test \#3 

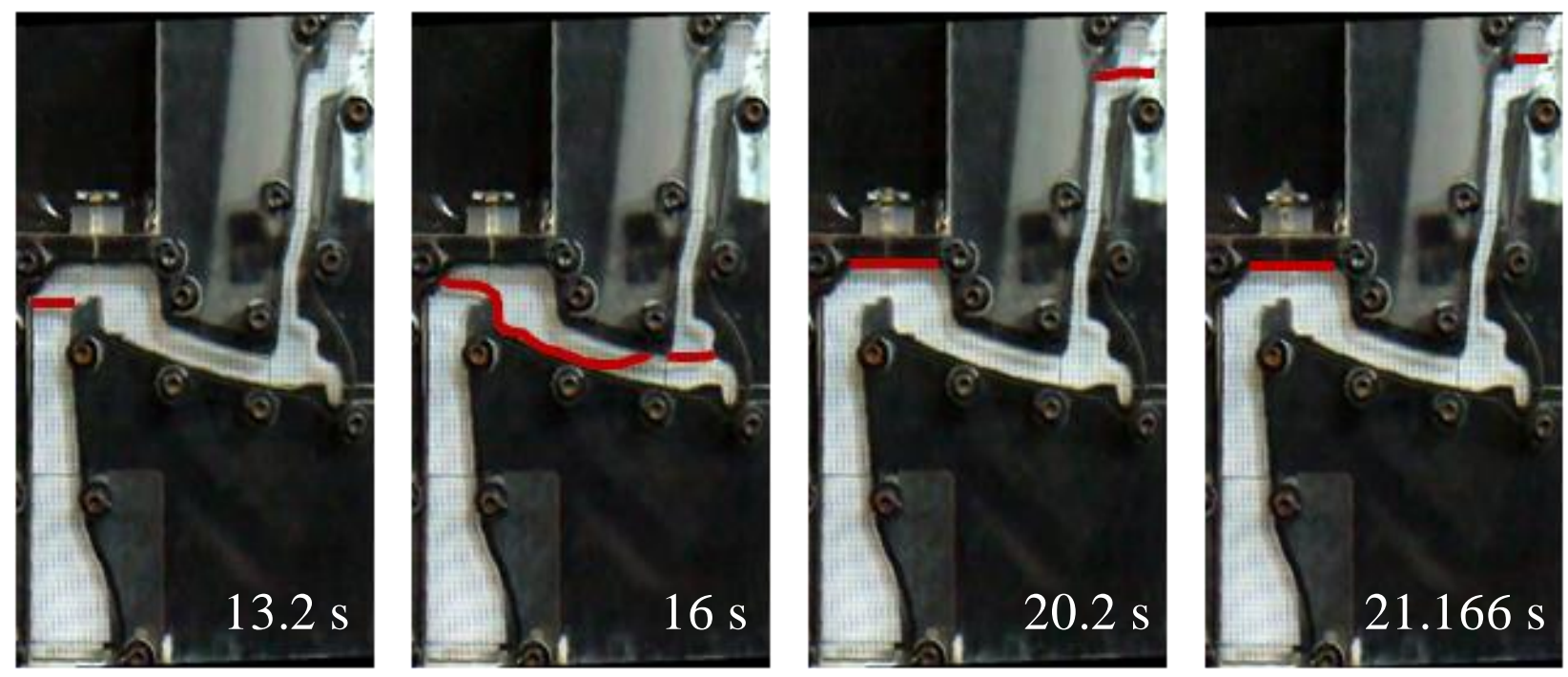

(a) Physical model
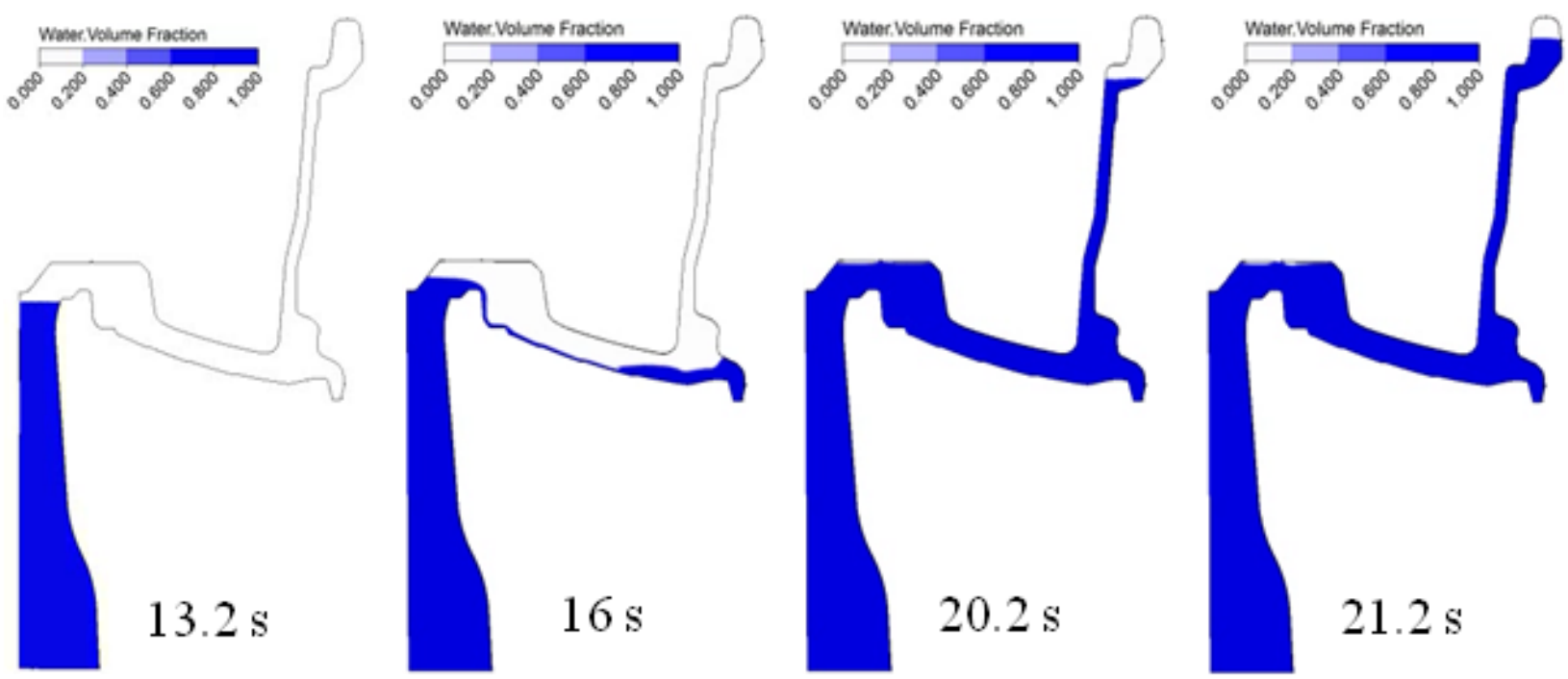

(b) Computational model

Figure 7.9. Comparison of the fill pattern between experimental result and the computational result for Test \#4 

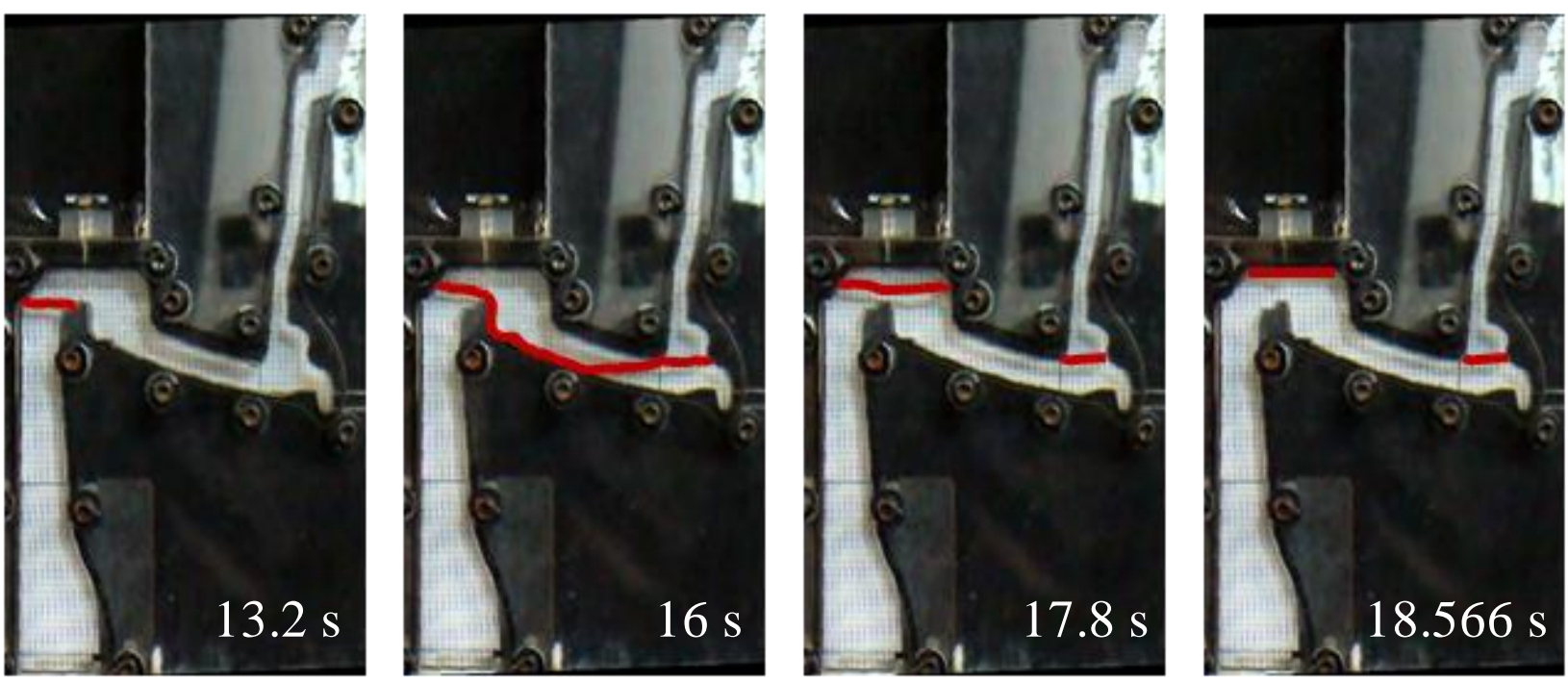

(a) Physical model
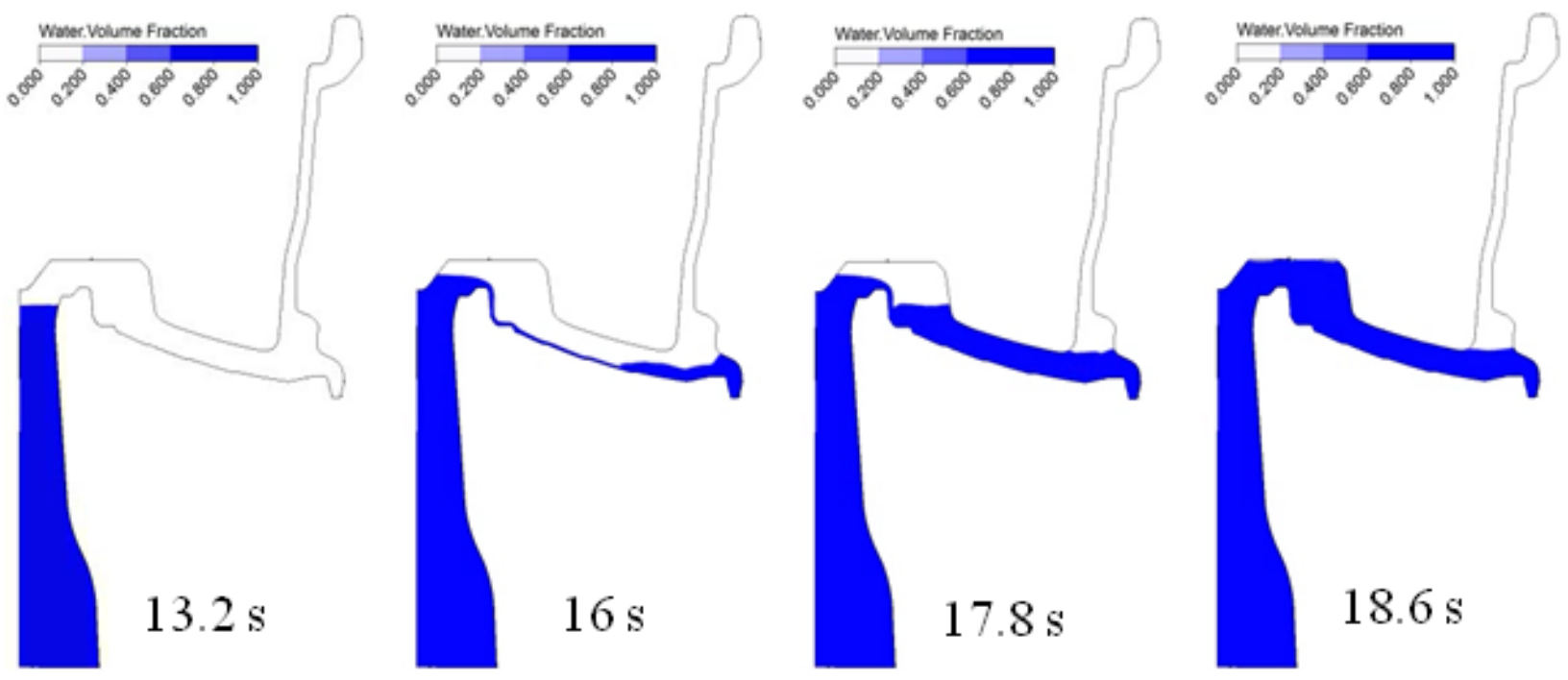

(b) Computational model

Figure 7.10. Comparison of the fill pattern between experimental result and the computational result for Test \#5

Table 7.2. Vent resistance coefficients for mode ls with diffe rent vent combinations

\begin{tabular}{ccc}
\hline Test \# & K perm $\left(\mathrm{m}^{2}\right)$ & K loss $\left(\mathrm{m}^{-1}\right)$ \\
\hline $\begin{array}{c}\text { \#1,\#2,\#3, \#4 (resistance } \\
\text { lowered 75\%) }\end{array}$ & $5.34 \times 10^{-10}$ & $292,013.0$ \\
\#5 (resistance decreased 90\%) & $1.34 \times 10^{-09}$ & $116,805.1$ \\
\hline
\end{tabular}


Despite the fact that the vent resistance coefficients of Test \#5 had to be further adjusted to obtain a comparable result to the experiment, the filling model developed in this project agrees well with the experimental filling under various venting conditions. Its robustness and accuracy has thus been validated.

\subsection{Effect of Inlet Pressure}

As seen in Section 4.1.3 Pressure Control System, the pressure in the experimental system is controlled by an electronic valve using feedback from a pressure sensor. The sensor measures the pressure at the bottom of the transition pipe. This information is converted to the pressure at the bottom of the die, and is used as the inlet pressure boundary conditions for the computational models. An inaccurate reading of the pressure will result in an inaccurate inlet boundary condition. And since the inlet pressure affects the flow rate, it could change the fill pattern, and provide unreliable modeling results.

The accuracy of the DAQ and pressure sensor affects the accuracy of the pressure measurements. To evaluate the error of the DAQ system used in this study, the liquid head pressures of two heights have been measured statically. One is $20 \mathrm{~mm}$ above the sprue entrance, which is the initial condition for all physical and computational models. This height corresponds to a liquid head pressure of $3.415 \mathrm{kPa}$ at the bottom of the transition pipe. The other is $441.4 \mathrm{~mm}$, which is the height difference between the top of the die and its bottom, and this height corresponds to a liquid head pressure of $7.540 \mathrm{kPa}$, measured at the bottom of the transition pipe. The reason for choosing a static liquid height is that it provides an exact value for liquid head pressure. The pressure of each height has been measured three times, and over 400 samples have been collected each time. The largest deviations from the calculated liquid head pressure were found in the measurements of the height $441.1 \mathrm{~mm}$. The maximum measured pressure is $7.564 \mathrm{kPa}, \mathrm{a}$ 
$0.32 \%$ increase to the calculated liquid head pressure, and the minimum measured pressure is $7.523 \mathrm{kPa}$, a $0.23 \%$ decrease.

To assess whether the error of the DAQ system affects the computational results, and also to obtain a preliminary perception of how sensitive the fluid flow is to the inlet pressure, the baseline pressure curve was both increased by $24 \mathrm{~Pa}(0.4 \%$ of the experimentally measured pressure range) and decreased by $17 \mathrm{~Pa}(0.29 \%$ of the experimentally measured pressure range). Models with these modified pressure curves were run, and the results were plotted with both the experimental and the baseline free surface heights in Figure 7.11. All models employed the 75\% lowered vent resistance coefficients.

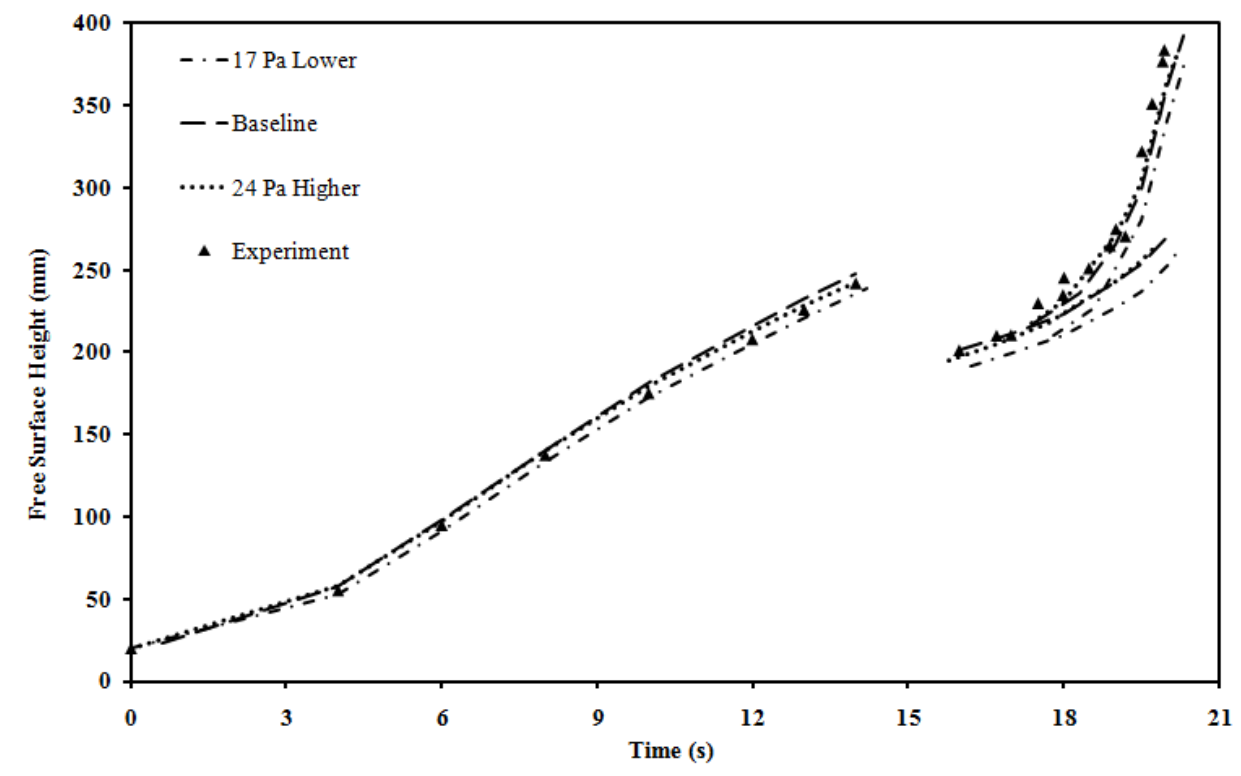

Figure 7.11. Comparison of the free surface height between computational models with diffe rent inlet pressure boundary and the physical model, showing the results of Test \#1

Interestingly, adding $24 \mathrm{~Pa}$ to the inlet pressure values does not increase the flow rate, although the model with inlet pressure decreased by $17 \mathrm{~Pa}$ fills slower. Nonetheless, the predictions curves are all close to each other and no significant effect from the inlet pressure was observed. This finding was further investigated by plotting the pressure evolution at three points in the domain, 
as shown in Figure 7.12. The predicted pressure curves for different inlet pressures conditions are almost identical to each other. It clearly shows that the internal pressure field was not affected when the inlet pressure was increased by $24 \mathrm{~Pa}$, or decreased by $17 \mathrm{~Pa}$, which confirms that the range in error in the pressure measurement should not influence the accuracy of the results.
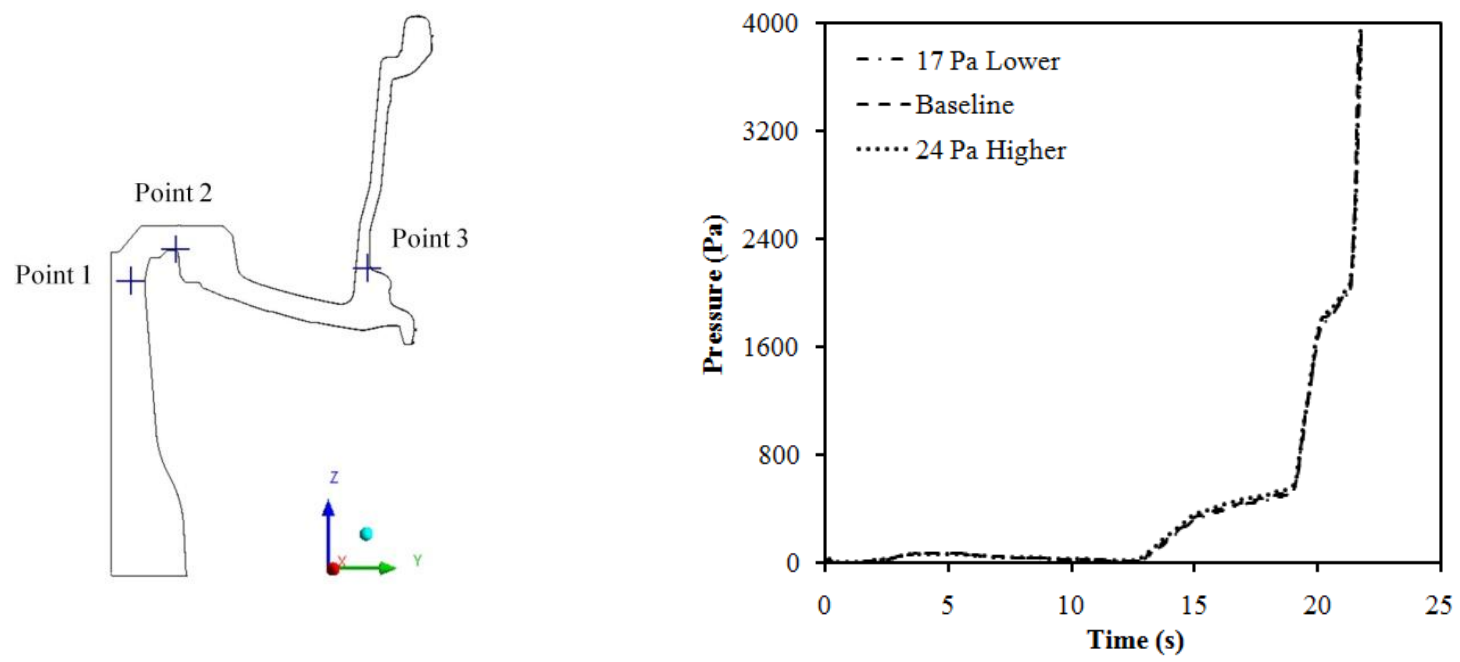

(a) The three points used to examine the static pressure

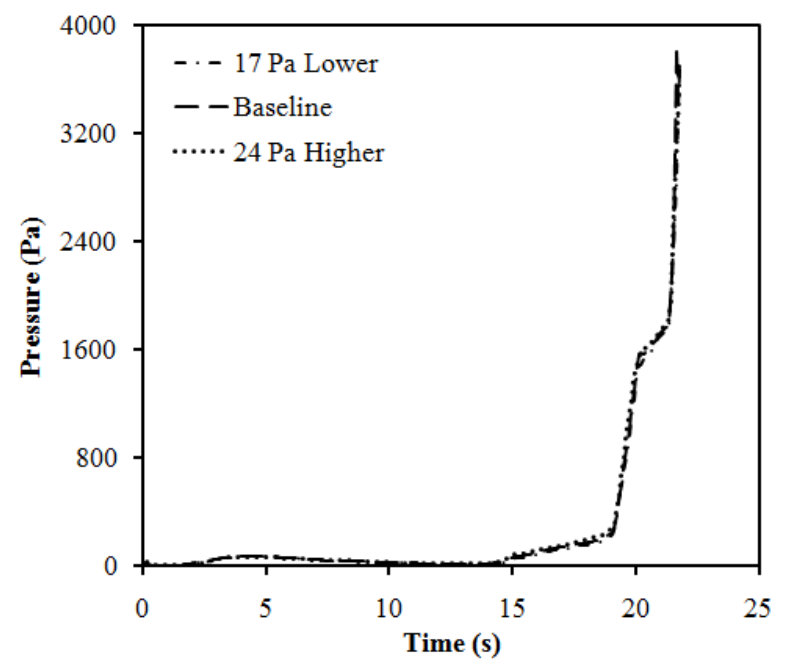

(c) Pressure plot at Point 2 (b) Pressure plot at Point 1

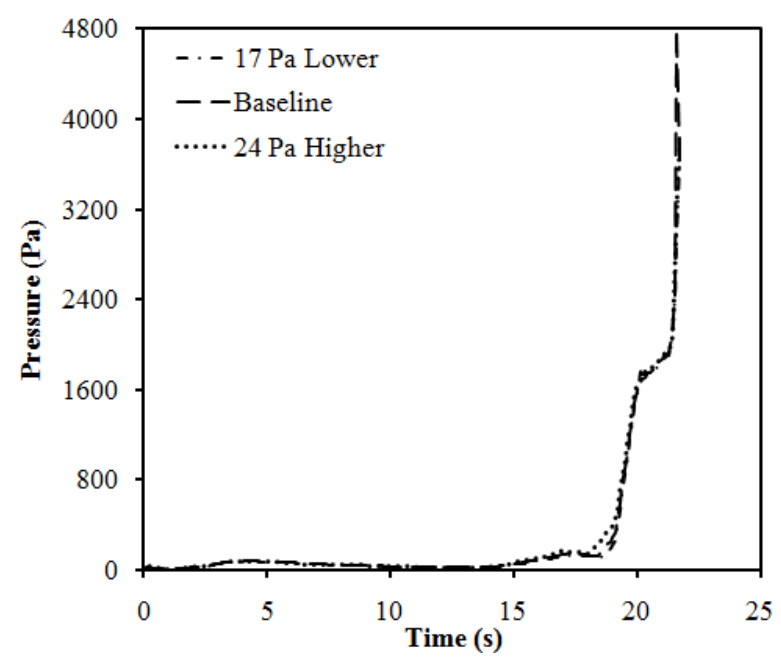

(d) Pressure plot at Point 3

Figure 7.12. Comparison of static pressure at three points in the wheel domain 


\section{SUMMARY AND CONCLUSION}

In this study, a water analogue physical model was developed to simulate liquid metal flow behavior during die filling of a low-pressure die-cast aluminum automotive wheel, with a view to understanding the free surface behavior and the formation of entrainment related defects. To allow for straightforward observation of the free surface and ease of construction, the physical model was based on a 2D planar die section rather than a 3D replica of the die cavity of a production automotive wheel. A computational model based on the commercial computational fluid dynamics code ANSYS CFX was also developed for the purpose of assessing the ability of a computational model to predict the flow conditions during die filling and to identify key features in model formulation that impact on the accuracy of the model. The reduced mesh complexity and run-time afforded by the $2 \mathrm{D}$ planar die section was another motivation for its adoption over a full 3D physical model.

The following points summarize the main conclusions from the experimental work:

1. The fluid flow in the LPDC casting system studied is relatively tranquil in the sprue and rim regions of the die and therefore the flow in these regions is unlikely to result in entrainment of free surface oxide films in commercial practice and the formation of entrainment related defects.

2. In the geometry studied, air was entrained at the point when the leading edge of the fluid flowing down the spoke intersects the outboard rim flange and during filling of the flange. In commercial practice the air entrained in this region may lead to the formation of oxide defects.

3. A persistent returning wave is likely to form in any spoke with a significant negative slope due to the reflection of fluid from the wall of the outboard rim flange. Air was entrained 
below the free surface of the returning wave and may cause oxide film related defects in commercial wheels.

4. Fold-in events were clearly seen in the free surface as it filled the spoke adjacent to the hub and the hub. These flow phenomena may be associated with cracks observed in the hub region of a wheel.

5. Tests with different combinations of vents in the water physical model showed that the hub and the riser vents are more important in ensuring the cavity is fully filled than the flange vents.

6. Vent resistance was shown to be critical in controlling the filling characteristics of the die. When designing dies for the LPDC process venting is an important design parameter as it may have a bearing on free surface turbulence and entrainment related defect formation. More work is clearly needed to understand the venting characteristics of commercial dies.

The following points summarize the main conclusions from the computational modeling work:

1. A computational model was developed and validated against experimental data obtained from the physical water model. The validation was based on comparison to the variation in free surface height with time, free surface geometry and the entrainment of air bubbles. In all cases, with the exception of the entrainment of some small bubbles, the computational model was able to qualitative reproduce the behavior observed in the water model.

2. The baseline or initial vent resistance coefficients applied in the computational model were estimated using a computational study based on a crude approximation of the actual vent geometry used in the experimental die. Additional tuning was required to properly define the vent resistance coefficients, which was consistent with actual vent geometries.

3. The results showed that the filling pattern including the free surface behavior was very sensitive to the vent resistance. The implication being that vent design in commercial die 
design may influence entrainment related defect formation. In addition, this conclusion points to the need for a full two-phase model analyses of low pressure die casting in order that the effect of backpressure is properly accounted for.

4. Sensitivity studies were undertaken on several factors related to the computational model such as the mesh size and the time step size and on whether or not a turbulent model was necessary. In terms of mesh size and time step size, the combination of $1 \mathrm{~mm}$ mesh size with a $0.01 \mathrm{~s}$ time step size was able to give accurate results with affordable computational load. In terms of the fluid model, the turbulent model was found to be unnecessary since it had a minimal impact on the flow behavior but took $28 \%$ more time to calculate.

5. Additional sensitivity analyses were undertaken on the fluid properties including surface tension and on whether or not wall adhesion (wetting) was needed. In terms of surface tension, a $\pm 6 \%$ change in surface tension did not affect the filling pattern, however, excluding the surface tension force from the model led to unreliable results. In terms of wall adhesion, the inclusion of wall adhesion force raised some numerical issues though it did give a better characterization of the free surface shape at the end of filling.

As a proviso, it is important to point out that the conclusions drawn from this work are based on results obtained from a $2 \mathrm{D}$ planar section water physical model and a computational model. Extrapolation to the conditions in a commercial3D should be done with care.

\subsection{Recommendations for Future Work}

The work presented offers an alternate, convenient and cost-effective way to study the conditions leading to free surface related defects formed during the die filling process of a LPDC automotive wheel, however there is still additional research required in this field to complete the understanding of the LPDC filling process. These required areas of research include: 


\section{Experimental:}

1. Since the venting conditions appear to play an important role in die filling, some work should be done to investigate the venting conditions present in commercial dies in order to ensure that future modeling work (both physical and numerical) is closely aligned with the industrial conditions. For example, the backpressure could be measured at discrete locations in the die cavity to quantitatively measure the internal pressure field in the die cavity. With this data in hand, a comparison could then be made with the results of a numerical analysis tuned via varying the vent resistance. The results would be an indirect measurement of venting capacity at different fill heights.

2. The pressure curve needs further examination both using the physical model and the computational model. For example, is it possible to adjust the pressure curve at critical times to avoid free surface entrainment?

3. Improvements could be made to the flow visualization associated with the physical model to enable more quantitative assessment of the flow behavior. Options that could be explored include Particle Image Velocimetry (PIV) and Laser-induced Fluorescence (LIF). This will aid in generating internal velocity field.

4. More die configurations should be investigated. The die could be redesigned to allow for the easy removal of different parts of the die. This modular design would make the change of different geometries much easier.

Computational modeling:

1. Application of the model to the industrial process, i.e. a periodic section of the $3 \mathrm{D}$ wheel geometry with material properties for aluminum would be a logical next step in a trajectory to developing a model of the commercial process. This would allow a more accurate 
understanding of flow within the industrial die cavity and allow the causes of defects to be identified and understood.

2. Several parameters should be explored to quantitatively assess the potential of free surface entrainment. Examples include the instantaneous free surface length or the ratio of the free surface length to the remaining air volume, the excess free surface, and some dimensionless number such as Webber and Froude numbers. These could provide a more direct and quantitative assessment between the flow types seen and the severity of defect formation. 


\section{Bibliography}

[1] B. Zhang, D. M. Maijer and S. L. Cockcroft, "Development of a 3-D thermal model of the low-pressure die-cast (LPDC) process of A356 aluminum alloy wheels," Materials Science and Engineering A-Structural Materials Properties Microstructure and Processing, 464 (1-2) (2007), 295-305

[2] J. Campbell, "Entrainment defects," Materials Science and Technology, 22 (2) (2006), 127 145

[3] B. Zhang et al., "Casting defects in low-pressure die-cast aluminum alloy wheels, " JOM Journal of the Minerals, Metals and Materials Society, 57 (11) (2005), 36-43

[4] A. E. Miller, "Investigation of erosive-corrosive wear in the low pres sure die casting of aluminum A356" (Masters, University of British Columbia, 2005).

[5] CRC Handbook of Chemistry and Physics, 91st Edition (Cleveland, Ohio: CRC Press, 2010 2011)

[6] J. Campbell, Castings, 1st Edition (Oxford, Boston: Butterworth-Heinemann, 1991)

[7] J. Mi, R. A. Harding and J. Campbell, "Effects of the entrained surface film on the reliability of castings," Metallurgical and Materials Transactions a-Physical Metallurgy and Materials Science, 35A (9) (2004), 2893-2902

[8] R. A. Harding, "Towards more reliable investment castings, " Int.J.Cast Met.Res., 19 (5) (2006), 289-301 
[9] D. Apelian, "Metal filtration: a critical review and update." Electr. Furn. Conf. Proc., 46 (1989), 375-389

[10] T. K. Loughnane, D. P. K. Singh and D. L. Zhang, "Quantify ing the effects of filtration on the quality of low pressure permanent mold cast aluminum alloy wheels." Trans. Am. Foundry Soc., 111 (2003), 303-314

[11] G. Hartmann, R. Seefeldt and J. Franke, "Practical Application of Next Generation Numerical Optimisation to HPDC Casting Process," http://www.magmasoft.de/ms/_data/PracticalApplicationOf_Next_Generation.pdf

[12] R. M. McDavid and J. A. Dantzig, "Fluid flow in casting rigging systems: modeling, validation, and optimal design," Metall.Mater.Trans.B, 29B (3) (1998), 679-690

[13] Swift R.E., Jackson J.H. and Eastwood L.W, "A study of principles of gating," AFS Transactions, 93 (1949), 76-88

[14] C. N. Cochran, D. L. Belitskus and D. L. Kinosz, "Oxidation of aluminum-magnesium melts in air, oxygen, flue gas, and carbon dioxide," Metall.Trans., B, 8B (2) (1977), 323-332

[15] M. V. Mal'tsev, Y. D. Chistyakov and M. I. Tsypin, "Electron-diffraction investigation of oxide films, formed on liquid aluminum and its alloys." Bull. Acad. Sci. USSR, Phys. Ser. (Engl. Transl.), 20 (1956), 747-750

[16] S. Impey, D. J. Stephenson and J. R. Nicholls, "The influence of surface preparation and pretreatments on the oxidation of liquid aluminum and aluminum-magnesium alloys," Microsc.Oxid.2, Proc.Int.Conf., 2nd, (1993), 338-346 
[17] S. A. Impey, D. J. Stephenson and J. R. Nicholls, "Mechanism of Scale Growth on Liquid Aluminum," Materials Science and Technology, 4 (12) (1988), 1126-1132

[18] S. Impey, D. J. Stephenson and J. R. Nicholls, "A study of the effect of magnesium additions on the oxide growth morphologies on liquid aluminum alloys," Microsc.Oxid., Proc.Int.Conf., 1st, (1991), 238-244

[19] I. Haginoya and T. Fukusako, "Oxidation of Molten Al-Mg Alloys," Transactions of the Japan Institute of Metals, 24 (9) (1983), 613-619

[20] W. Thiele, "The oxidation of melts of aluminum and of aluminum alloys," Aluminium, 38 (1962), 707-15,780-6

[21] O. Kubaschewski and B. E. Hopkins, Oxidation of metals and alloys (London: Butterworths, 1962)

[22] W. C. Sleppy, "Oxidation of Molten High-Purity Aluminum in Dry Oxygen," J.Electrochem.Soc., 108 (12) (1961), 1097-1102

[23] J. Campbell, Castings, 2nd Edition 2nd Edition (Oxford, Boston: Butterworth-Heinemann, 2003)

[24] R. Raiszadeh and W. D. Griffiths, "A method to study the history of a double oxide film defect in liquid aluminum alloys," Metallurgical and Materials Transactions B-Process Metallurgy and Materials Processing Science, 37 (6) (2006), 865-871

[25] A. K. M. B. Rashid and J. Campbell, "Oxide defects in a vacuum investment-cast Ni-based turbine blade." Metall. Mater. Trans. A, 35A (7) (2004), 2063-2071 
[26] J. Campbell, "Evidence for entrainment defects," Materials Science and Technology, 24 (7) (2008), 875-879

[27] J. Campbell, "An overview of the effects of bifilms on the structure and properties of cast alloys," Metallurgical and Materials Transactions B-Process Metallurgy and Materials Processing Science, 37 (6) (2006), 857-863

[28] Q. G. Wang et al., "Oxide films, pores and the fatigue lives of cast aluminum alloys," Metallurgical and Materials Transactions B-Process Metallurgy and Materials Processing Science, 37 (6) (2006), 887-895

[29] J. F. Major, "Porosity control and fatigue behavior in A356-T61 aluminum alloy." Trans. Am. Foundrymen's Soc., 105 (1997), 901-906

[30] C. H. Caceres and B. I. Selling, "Casting defects and the tensile properties of an Al-Si-Mg alloy," Materials Science and Engineering a-Structural Materials Properties Microstructure and Processing, 220 (1-2) (1996), 109-116

[31] N. R. Green and J. Campbell, "Influence of oxide film filling defects on the strength of Al7Si-Mg alloy castings." Trans. Am. Foundrymen's Soc., 102 (1995), 341-347

[32] M. C. Flemings, Solidification processing (New York: McGraw-Hill, 1974)

[33] K. Li and E. Chang, "Mechanism of nucleation and growth of hydrogen porosity in solidifying A356 aluminum alloy: an analytical solution." Acta Mater., 52 (1) (2004), 219-231

[34] J. C. Fisher, "The Fracture of Liquids," J.Appl.Phys., 19 (11) (1948), 1062-1067 
[35] L. Yao et al, "Modeling of porosity size distribution in A356 tapered cylinder castings." (Section Title: Nonferrous Metals and Alloys 2009)

[36] L. Yao et al, "Effect of oxide films on microporosity formation in A356 aluminum alloy castings" (Conference of Metallurgists Vancouver, Canada, 2010)

[37] J. A. Taylor, G. B. Schaffer and D. H. St. John, "Unsolicited outbreaks of porosity in aluminum - silicon based alloys." (Section Title: Nonferrous Metals and Alloys 1995)

[38] X. -. Chen, R. I. L. Guthrie and J. E. Gruzleski, "Quantitative measurement of melt cleanliness in aluminum-silicon casting alloys." (Section Title: Nonferrous Metals and Alloys 1995)

[39] G. Ran, J. Zhou and Y. Wang, "Study on tensile properties and fractography of cast A356 aluminum alloy." Xiyou Jinshu Cailiao Yu Gongcheng, 35 (10) (2006), 1620-1624

[40] J. A. Francis and G. M. D. Cantin, "The role of defects in the fracture of an Al-Si-Mg cast alloy." Mater. Sci. Eng., A, A407 (1-2) (2005), 322-329

[41] A. R. Mirak et al., "Effect of in-gate velocity of perfectness and mechanical properties of magnesium casting alloy (AZ91C)." Nashriyyah-i Danishkadah-i Fanni, 40 (7) (2007), 981 Persian-990 Persian, 4 English - 5 English

[42] J. Mi et al., "Entrained oxide films in TiAl castings." Intermetallics, 11 (4) (2003), 377-385

[43] S. Shivkumar, L. Wang and R. Lavigne, "Quantitative evaluation of pore characteristics in cast aluminum alloys." Light Met. (Warrendale, Pa.), (1993), 829-838 
[44] G. Laslaz and P. Laty, "Gas porosity and metal cleanliness in aluminum casting alloys." Trans. Am. Foundrymen's Soc., 99 (1991), 83-90

[45] D. Dispinar and J. Campbell, "Critical assessment of reduced pressure test. Part 1: Porosity phenomena," International Journal of Cast Metals Research, 17 (5) (2004), 280-286

[46] T. W. Clyne, M. Wolf and W. Kurz, "The effect of melt composition on solidification cracking of steel, with particular reference to continuous casting." Metall. Trans., B, 13B (2) (1982), 259-266

[47] M. Rappaz, J. -. Drezet and M. Gremaud, "A new hot-tearing criterion." Metall. Mater. Trans. A, 30A (2) (1999), 449-455

[48] P. S. Mohanty and J. E. Gruzleski, "Mechanism of grain refinement in aluminum." Acta Metall. Mater., 43 (5) (1995), 2001-2012

[49] P. S. Mohanty, Studies on the mechanisms of heterogeneous nucleation of grains and pores in aluminum castings (Montreal: McGill University, Department of Mining and Metallurgical Engineering, 1994)

[50] J. Runyoro, S. M. A. Boutorabi and J. Campbell, "Critical gate velocities for film-forming casting alloys: A basis for process specification." Trans. Am. Foundrymen's Soc., 100 (1992), 225-284

[51] Carl Reilly, "Development of Quantitative Casting Quality As sessment Criteria Using Process Modelling" (Ph.D. thesis, The University of Birmingham, 2010), 4-36. 
[52] M. Cox, R. A. Harding and J. Campbell, "Optimised running system design for bottom filled aluminium alloy 2L99 investment castings." Mater. Sci. Technol., 19 (5) (2003), 613-625

[53] B. S. Massey, Mechanics of fluids, 6th Edition (New York: Van Nostrand Reinhold Co., 1989)

[54] C. Reilly et al, "Using the calculated froude number for quality assessment of casting filling methods." (Section Title: Nonferrous Metals and Alloys 2009)

[55] C. Reilly, N. R. Green and M. R. Jolly, "Surface Oxide Film Entrainment Mechanisms in Shape Casting Running Systems." Metall. Mater. Trans. B, 40B (6) (2009), 850-858

[56] B. Sirrell, M. Holliday and J. Campbell, "The benchmark test 1995." (Section Title: Nonferrous Metals and Alloys 1995)

[57] J. Campbell, "Thin wall castings," Materials Science and Technology, 4 (1988), 194-204(11)

[58] J. Evans, J. Runyoro and J. Campbell, "The flow of liquid aluminum in thin section castings." (Section Title: Nonferrous Metals and Alloys 1997)

[59] Y. Chung and A. W. Cramb, "Dynamic and equilibrium interfacial phenomena in liquid steel-slag systems." Metall. Mater. Trans. B, 31B (5) (2000), 957-971

[60] D. P. K. Singh et al., "Die design strategy for improved productivity and quality in die casting." Trans. Am. Foundrymen's Soc., 107 (1999), 127-133

[61] Bernd Meurer, Dirk Haferkamp and Alexander Jörg, "Use of simulation in the prod uction of cast aluminium wheels," http://www.magmasoft.de/ms/_data/UseOfSimCastAlWheelsCP_T2001.pdf 
[62] B. Sirrell and J. Campbell, "Mechanism of filtration in reduction of casting defects due to surface turbulence during mold filling." Trans. Am. Foundrymen's Soc., 105 (1997), 645-654

[63] D. Su and Y. Zhao, "Simulation of filling and solidification of aluminum alloy wheel in low pressure die casting." Tezhong Zhuzao Ji Youse Hejin, 27 (9) (2007), 682-684

[64] I. Ohnaka, "Recent development of numerical modeling of casting and solidification." (Section Title: Nonferrous Metals and Alloys 2003)

[65] L. Jia, S. Xiong and B. Liu, "Study on numerical simulation of mold filling and heat transfer in die casting process." J. Mater. Sci. Technol. (Shenyang, China), 16 (3) (2000), 269-272

[66] H. Zhao, I. Ohnaka and J. Zhu, "Modeling of mold filling of Al gravity casting and validation with X-ray in-situ observation," Appl.Math.Model., 32 (2) (2008), 185-194

[67] J. Campbell, "Solidification modeling: current limitations and future potential." Mater. Sci. Technol., 7 (10) (1991), 885-894

[68] I. Ohnaka et al, "Improvement of mold filling simulation" (Section Title: Nonferrous Metals and Alloys 2009)

[69] I. Ohnaka, M. Ohmasa and T. Takeuchi, "Comparison of measured and simulated fluid flow in low-pressure die casting." (Section Title: Nonferrous Metals and Alloys 1991)

[70] A. Kimatsuka et al., "Mold filling simulation with consideration of gas escape through sand mold." Int. J. Cast Met. Res., 15 (3) (2002), 149-152

[71] J. Jakumeit, K. Goodheart and M. Albers, "Influence of the gas phase on mold filling for sand casting" 2009) 
[72] J. Campbell, "The modeling of entrainment defects during casting." (Section Title: Nonferrous Metals and Alloys 2006)

[73] X. Yang et al., "Numerical modelling of entrainment of oxide film defects in filling of aluminium alloy castings." Int. J. Cast Met. Res., 17 (6) (2004), 321-331

[74] N. W. Lai, W. D. Griffiths and J. Campbell, "Modelling of the potential for oxide film entrainment in light metal alloy castings" (Modeling of Casting, Welding and Advanced Solidification Processes X, Proceedings of the International Conference on Modeling of Casting, Welding and Advanced Solidification Processes, 10th, Destin, FL, United States, 2003)

[75] R. Cuesta et al., "Numerically modeling oxide entrainment in the filling of castings: The effect of the webber number," JOM Journal of the Minerals, Metals and Materials Society, 58 (11) (2006), 62-65

[76] T. Nguyen and J. Carrig, "Water analogue studies of gravity tilt casting copper alloy components," Transactions of the American Foundrymen's Society, 94 (1986), 519-528

[77] M. Forte, D. Bouchard and A. Charette, "Flow visualization of Newtonian and nonNewtonian fluids in high pressure die casting." (Section Title: Nonferrous Metals and Alloys 2006)

[78] R. Cuesta et al., "Water analogue experiments as an accurate simulation method of the filling of gravity castings," Trans.Am.Foundry Soc., 114 (2006), 137-150

[79] Z. Zhao et al., "Computer video technology of water simulation for aluminum liquid filling process." Zhongguo Youse Jinshu Xuebao, 15 (8) (2005), 1262-1266 
[80] M. Schmid and F. Klein, "Experimental investigation of mold filling in high-pressure die casting." (Section Title: Nonferrous Metals and Alloys 1998)

[81] N. D. Perkins and C. J. Bain, "transparent water models as an aid to gating design for permanent mould," J. Aust. Inst. Metals., 10 (1965), 160-168

[82] R. M. McDavid and J. A. Dantzig, "Experimental and numerical investigation of mold filling." (Section Title: Nonferrous Metals and Alloys 1998)

[83] J. Mi, R. A. Harding and J. Campbell, "Effects of the entrained surface film on the reliability of castings." Metall. Mater. Trans. A, 35A (9) (2004), 2893-2902

[84] Swagelok, "Valve Sizing," http://www.swagelok.com/downloads/webcatalogs/EN/MS-0684.PDF

[85] Hass Manufacturing Company, http://www.has smfg.com/search.pl/1256787027$\underline{30199 ? \text { keyword=1044\&submit=Search }}$

[86] Freescale Semiconductor, http://cache.freescale.com/files/sensors/doc/data_sheet/MPVZ5010G.pdf?fpsp=1\&WT_TYPE= $\underline{\text { Data }}$

$\underline{\text { Sheets } \& W T \text { VENDOR=FREESCALE } \& W T \text { FILE_FORMAT=pdf\&WT_ASSET=Documentati }}$ $\underline{\text { on }}$

[87] C. A. Smith and A. B. Corripio, Principles and practice of automatic process control, 3rd Edition (Hoboken, NJ: Wiley, 2006) 
[88] National Instruments Corporation, "LabWindows/CVI PID Control Toolkit User Manual," http://www.ni.com/pdf/manuals/371685c.pdf

[89] ANSYS CFX Release 12.0 Documentation, Inc ANSYS. , 2009

[90] K. Avila et al., "The Onset of Turbulence in Pipe Flow," Science, 333 (6039) (2011), 192196

[91] F. M. Francisca, V. A. Rinaldi and J. C. Santamarina, "Instability of Hydrocarbon Films over Mineral Surfaces: Microscale Experimental Studies." J. Environ. Eng. (Reston, VA, U. S.), 129 (12) (2003), 1120-1128

[92] R. B. Bird, W. E. Stewart and E. N. Lightfoot, Transport phenomena Rev 2 (New York, NY: John Wiley \& Sons, Inc., 2007) 